\title{
Analysis of the sleep homeostat of the nematode Caenorhabditis elegans
}

\author{
Dissertation \\ for the award of the degree \\ "Doctor rerum naturalium" (Dr. rer. nat.) \\ Division of Mathematics and Natural Sciences \\ of the Georg-August-Universität Göttingen
}

submitted by

Jan-Philipp Spies

from Hamburg, Germany

Göttingen 2014 



\section{Members of the Thesis Committee:}

Dr. Henrik Bringmann

(Reviewer)

Prof. Dr. Andreas Stumpner (Reviewer)

Prof. Dr. Jens Frahm
Max Planck Research Group Sleep and Waking,

Max Planck Institute for Biophysical Chemistry, Göttingen

Department of Neurobiology,

Georg-August-Universität Göttingen

Biomedical NMR,

Max Planck Institute for Biophysical Chemistry, Göttingen

\section{Date of the oral examination:}

20.02.2015 



\section{Affidavit}

Hereby, I declare that the presented thesis entitled Analysis of the sleep homeostat of the nematode C. elegans was written entirely by myself and that I have only used the sources and materials cited.

Göttingen, 29.12.2014

Jan-Philipp Spies 



\section{Summary}

Sleep is essential for animal life and conserved in all animals that have a nervous system. The nematode Caenorhabditis elegans exhibits sleep that is regulated homeostatically during larval development. However its regulation is poorly understood. The aim of this thesis was to gain a better understanding of the neuronal and molecular mechanisms underlying sleep homeostasis. To investigate the sleep homeostat of $C$. elegans, I developed an automated sleep deprivation setup based on mechanical stimulation/optogenetics allowing simultaneous behavioral analysis and functional imaging of neurons. I have identified RIS as the neuron reflecting the sleep homeostat and several molecules underlying sleep using genetic approaches and in vivo neuronal imaging.

I found that sleep deprivation significantly shortens the animals sleeping duration and yields persistent increased locomotion past the deprivation period. To look for homeostatic changes in sleep intensity, I investigated the effect of sleep deprivation on the mechanosensory neuron ALM using calcium imaging. Prolonged sleep deprivation induces a significant decrease in stimulus-evoked ALM calcium transients compared to a wake control. In addition to this homeostatic regulation of ALM responsiveness I found that quiescence is already induced while the deprivation stimulus still yields strong activation of ALM. I show that this quiescence is induced by the interneuron RIS. Hence, RIS reflects the homeostat actively driving quiescence as a response to sleep deprivation. Moreover I demonstrate that RIS has a more general function on the homeostatic regulation of locomotion. Both spontaneous locomotion and evoked locomotion are dampened by RIS activation. The fact that RIS controls both the balance between mobility and immobility and the sleep homeostat suggests a related evolutionary origin.

By reverse genetic approaches I found that the transcription factor lim- 6 which is known to regulate RIS differentiation, is required for wild-type sleep. However, RIS activity at sleep onset and in response to sleep deprivation is similar to wild-type.

I demonstrate that the neurotransmitters serotonin, dopamine, GABA, glutamate, and octopamine are not individually essential for the sleep homeostat. Furthermore I found that GABA, glutamate, and octopamine knockout mutants do not show altered RIS activity. egl-3 knockout mutants which have defects in the synthesis of neuropeptides, show RIS overactivation in the mid and late sleep phase while barely immobilizing during sleep. This implies that neuropeptide signaling is required downstream of RIS for the induction of quiescence.

Finally, ion channel modulation is widely associated with sleep and sleep homeostasis. I demonstrate that the inositol trisphosphate receptor itr-1(sa73) KO mutant, has a reduced RIS sleep onset peak. I show that the calcium channel $\alpha 2 / \delta$ subunit unc-36 knockout mutant exhibits long lasting RIS overactivation during sleep. 



\section{Contents}

1 Introduction 1

1.1 Sleep . . . . . . . . . . . . . . . . . . . . . 1

1.1.1 Functions of sleep . . . . . . . . . . . . . . . . 1

1.1.2 Circadian regulation of sleep . . . . . . . . . . . . . 2

1.1.3 Homeostatic regulation of sleep . . . . . . . . . . . . . . . 3

1.1 .4 Conservation of sleep $\ldots \ldots \ldots \ldots \ldots$

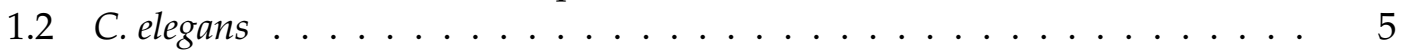

1.2.1 C. elegans sleep . . . . . . . . . . . . . . . . 6

1.2.2 Sleep homeostasis in C. elegans . . . . . . . . . . . 7

1.3 Thesis Aims ........................... 9

2 Methods 11

2.1 C. elegans maintenance . . . . . . . . . . . . . . . . . . . . 11

2.2 Creating transgenic C. elegans strains . . . . . . . . . . . . . . . . 11

2.2.1 Plasmid construction by Gateway Cloning ${ }^{\mathrm{TM}} \ldots \ldots \ldots$. . . . . . . 11

2.2.2 Microinjection and microparticle bombardment . . . . . . . . . 11

2.2.3 Crossings of C. elegans strains . . . . . . . . . . . . . . . . 12

2.2 .4 PCR genotyping . . . . . . . . . . . . . . . . . . . 12

2.3 Calcium imaging and optogenetics . . . . . . . . . . . . . . 12

2.3 .1 In vivo calcium imaging . . . . . . . . . . . . . . . . . . . . . . . . . . . . . . .

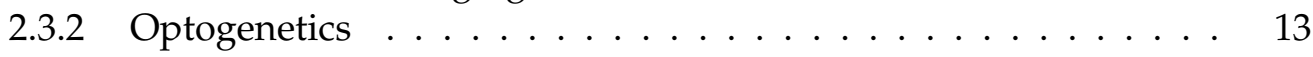

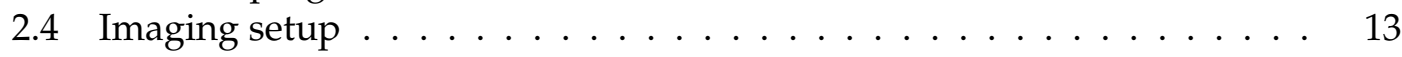

2.4 .1 Microfluidic device . . . . . . . . . . . . . . . . 13

2.4.2 Differential interference contrast (DIC) and brightfield imaging . 13

2.4 .3 Fluorescence imaging . . . . . . . . . . . . . . . . . . . 14

2.4 .4 Spinning disc imaging . . . . . . . . . . . . . . . . . . 14

2.5 Experimental sleep deprivation setup . . . . . . . . . . . . . . . . 14

2.5.1 Microscope setup for simultaneous brightfield and fluorescence

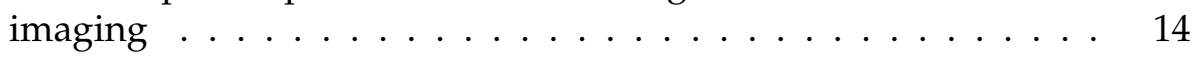

2.5.2 Automated sleep deprivation setup . . . . . . . . . . . . . 15

2.5.3 Mechanical stimulation by dish tapping . . . . . . . . . . . . . 15

2.5.4 Channelrhodopsin experiments . . . . . . . . . . . . . . 16

2.6 "C. elegans automated sleep deprivation" . . . . . . . . . . . . . . . . . . . . . 17

2.6 .1 Initialize measurement . . . . . . . . . . . . . . . . . . . . 19

2.6 .2 Image acquisition . . . . . . . . . . . . . . . . . 22

2.6 .3 Image processing . . . . . . . . . . . . . . 24 
2.6 .4 Sleep detection $\ldots \ldots \ldots \ldots \ldots$. . . . . . . . . . . . . . . . . . . . . 28

2.6 .5 Control TTL protocol . . . . . . . . . . . . . . . . . . 32

2.6 .6 Sleep deprivation . . . . . . . . . . . . . . . . . . . 33

2.6.7 Update image \& exit condition . . . . . . . . . . . . . . . 35

2.6 .8 Wait ........................ 36

2.6.9 "C. elegans automated sleep deprivation" interface . . . . . . . . . 36

2.6.10 Optimizing sleep deprivation . . . . . . . . . . . . . . . . 43

2.7 Analysis of behavioral and neuronal activity . . . . . . . . . . . . 45

2.8 Statistical analysis . . . . . . . . . . . . . . . . . . . . 45

2.9 Transcriptional profiling using RNA Sequencing . . . . . . . . . . . . 45

3 Results 47

3.1 Automated sleep recognition in C. elegans . . . . . . . . . . . . . . . . 47

3.1.1 Automated identification of the worm outline . . . . . . . . 48

3.1.2 Image subtraction as a readout for mobility . . . . . . . . . . . . 51

3.1.3 A sleep criterion based on image subtraction . . . . . . . . . . . 53

3.1.4 Evaluation of sleep detection using the image subtraction method 55

3.2 Automated sleep deprivation in C. elegans - Comparing ChR activation of neurons and mechanical stimulation by dish tapping. . . . . . . . . . 57

3.2.1 ChR2 light activation of neurons . . . . . . . . . . . . 57

3.2.2 Red shifted channelrhodopsin light activation of neurons - C1V1 \& Chrimson . . . . . . . . . . . . . . . . 63

3.2.3 Mechanical stimulation by dish tapping . . . . . . . . . . . . . . 64

3.2.4 Comparison of deprivation methods . . . . . . . . . . . . . . 66

3.3 Sleep deprivation by dish tapping . . . . . . . . . . . . 68

3.3.1 Detailed evaluation of sleep deprivation by dish tapping . . . . . 68

3.3.2 Effects of sleep deprivation on sleep duration . . . . . . . . . . 71

3.3.3 Behavioral effects of sleep deprivation . . . . . . . . . . . . 72

3.4 RIS activity is reflecting the sleep homeostat . . . . . . . . . . . . . 75

3.4.1 RIS activity in undeprived wild-type worms . . . . . . . . . . 75

3.4.2 RIS activity upon sleep deprivation . . . . . . . . . . . . 77

3.4.3 RIS activity during sleep following persistent stimulation during L1 wake . . . . . . . . . . . . . . . . 84

3.5 The sleep homeostat and mechanosensory neuron activity . . . . . . . 86

3.5.1 ALM sensory neuron responsiveness is reduced by prolonged sleep deprivation . . . . . . . . . . . . . . . . 86

3.5.2 The sleep homeostat drives quiescence while mechanosensory neurons still sense the deprivation stimulus . . . . . . . . . .

3.5.3 Homeostatic quiescence induction while sensory neurons still sense deprivation stimulus is mediated by RIS . . . . . . . . . . 88

3.6 Molecular and genetic basis of RIS signaling . . . . . . . . . . . . . . . . 91

3.6.1 The LIM homeobox transcription factor lim-6 . . . . . . . . . . . 91

3.6.2 Sleep phenotype of lim-6 mutants . . . . . . . . . . . . 92 
3.6.3 Rescue of the lim-6 mutant sleep phenotype . . . . . . . . . . . 95

3.6.4 RIS activity of undeprived lim-6 mutants . . . . . . . . . . . . . 98

3.6.5 Sleep deprivation and its effect on RIS activity in lim-6 mutants . 102

3.6.6 RNA Sequencing of lim-6 mutants . . . . . . . . . . . . . . . 102

3.6.7 Link between aptf-1, lim-6 and RIS . . . . . . . . . . . . . . . 104

3.6 .8 aptf-1 RIS phenotype . . . . . . . . . . . . . . . . . . 105

3.6 .9 Nuclear hormone receptors . . . . . . . . . . . . . . . . . . 107

3.6 .10 Neurotransmitters . . . . . . . . . . . . . . . . . . . . . . . . . . 109

3.6 .11 Neuropeptides . . . . . . . . . . . . . . . . . . . . . . . . . . 118

3.6.12 Subunits of voltage-gated calcium channels and an inositol trisphosphate receptor . . . . . . . . . . . . . . . . 121

3.7 Neuronal regulation of the sleep homeostat besides RIS . . . . . . . . . . 125

3.7.1 Search for sleep active neurons . . . . . . . . . . . . . . . . 125

3.7.2 RIA: A putative wake active neuron . . . . . . . . . . . . . . . 125

3.7.3 Neuronal activity downstream of RIS . . . . . . . . . . . . 126

4 Discussion 129

4.1 Development of the "C. elegans automated sleep deprivation" setup . . . 129

4.1 .1 Sleep detection . . . . . . . . . . . . . . . . . . . . . . . . . 129

4.1 .2 Sleep deprivation . . . . . . . . . . . . . . . . . . . . . . . . . . . . 129

4.2 Sleep deprivation shortens sleep duration and yields persistent increased locomotion past the deprivation period. . . . . . . . . . . . . . . 130

4.3 Homeostatic regulation of sensory responsiveness . . . . . . . . . . . . . 131

4.4 RIS is reflecting the sleep homeostat . . . . . . . . . . . . . . . . . 131

4.4 .1 Sleep promoting neuron . . . . . . . . . . . . . . . . 131

4.4.2 RIS overcomes arousal promoting neuronal signaling . . . . . . . 131

4.4 .3 RIS circuitry . . . . . . . . . . . . . . . . . . . . . . . . . . . 132

4.4.4 Locomotion origin of the sleep homeostat . . . . . . . . . . . . . . 132

4.4.5 Other mechanisms besides RIS that regulate sleep homeostasis in C. elegans . . . . . . . . . . . . . . . . . 132

4.5 Molecular and genetic mechanisms underlying the sleep homeostat . . . 133

4.5.1 Transcription factors and their role in the sleep homeostat - aptf-1 and $\lim -6$. . . . . . . . . . . . . . . . . . . . . . . . . . 133

4.5.2 Nuclear hormone receptors . . . . . . . . . . . . . . . . . . . 133

4.5 .3 Neurotransmitter signaling . . . . . . . . . . . . . . . . . . 134

4.5.4 Neuropeptide signaling and its function on the sleep homeostat . 134

4.5.5 Ion channels and their function on the sleep homeostat . . . . . . 134

4.6 Conclusion . . . . . . . . . . . . . . . . . . . . 135

$\begin{array}{lr}\text { Bibliography } & 137\end{array}$

$\begin{array}{lr}\text { Acknowledgements } & 147\end{array}$

$\begin{array}{lr}\text { Appendix } & 149\end{array}$ 
A Abbreviations and glossary . . . . . . . . . . . . . . . . 149

B C. elegans strains, constructs and primers used/generated during this thesis151

B.1 C.elegans strain list . . . . . . . . . . . . . . . . . . . . 151

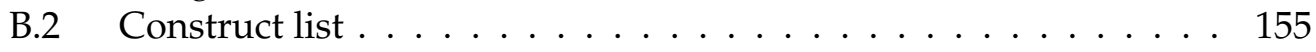

B.3 Primer list . . . . . . . . . . . . . . . . 156

C Dish tapping device for simultaneous/consecutive stimulation with different strength . . . . . . . . . . . . . . . . . . . . . 159

E Curriculum vitae . . . . . . . . . . . . . . . . . . 163 


\section{Chapter 1}

\section{Introduction}

\subsection{Sleep}

About one third of our lifetime we spend sleeping, but still there is no consensus about what precisely sleep is. Meanwhile, physiological and behavioral criteria are commonly used to define sleep. Since Pierons first definition in 1913, behavioral sleep criteria have been refined several times and led to the definition of Irene Tobler in the mid1980s [1], [2], [3]. The absence of voluntary movement (i), a sleep specific posture (ii) and an increased arousal threshold (iii). Furthermore it is quickly reversible upon stimulation (iv) and underlies homeostatic regulation (v).

In animals that have a neocortex, as birds and mammals, sleep can be defined by specific patterns of electrical brain activity. Electroencephalography (EEG) and magnetoencephalography (MEG) enable measuring the synchronized activity of thousands or millions of cortical neurons. Brain activity during sleep is distinct from wake. It can be divided into two main phases, rapid eye movement (REM) sleep and non-rapid eye movement (NREM) sleep. Characteristic for NREM sleep is the slow wave activity (SWS) of high voltage synchronized slow electrical field potential oscillations. While REM sleep, similar to wake, is associated with low voltage irregular EEG waves. High amplitudes of SWS correspond to deep NREM sleep. Long-term suppression of the SWS is lethal, whereas REM sleep suppression by medication, as used for treatment of depression, does not have disastrous effects in humans. During sleep the thalamic gate closes, cutting off the cortex from new sensory information.

\subsubsection{Functions of sleep}

The question if sleep serves a core function has yet to be answered. However progress on the function of sleep has been made on both cellular level (e.g. energy conservation) and systems level (e.g. memory consolidation). Everybody experiences sleep as the way to replenish energy resources. Indeed, energy consumption is reduced during sleep, as measured by glucose and ATP utilization, and energy stores (glycogen) get refilled [4], [5]. However energy conservation could be achieved by a simple resting state, without reduced arousal and altered consciousness. The fact that animals do require sleep immediately after hibernation implies that sleep serves other functions apart from ones related to energy metabolism [6]. Furthermore, sleep has been 
associated with the immune system and its response to cellular stress. Amount and intensity of NREM sleep increases as a reaction to immune challenge [7]. Even in the absence of immune challenge, pro inflammatory molecules promote sleep, whereas antiinflammatory molecules inhibit sleep [8].

It is scientific consensus that sleep serves important brain related functions. Behavioral studies in humans show the beneficial effect of sleep on memory consolidation [9], [10]. Memory consolidation refers to the process of stabilizing new memories encoded during wake and their integration into long term memory. There is a causal relationship between brain wave activity during sleep and memory consolidation that was demonstrated using transcranial electrical stimulation (TES). TES application of slowly oscillating electric potential fields, mimicking SWS, improves the consolidation of hippocampus dependent memories [11]. Memory consolidation on a neuronal level is achieved by changing synaptic connectivity and plasticity. It is hypothesized that two mechanisms change synaptic plasticity: global synaptic downscaling and selective local synaptic potentiation. Globally, over large cortical areas, synaptic downscaling occurs during REM and NREM sleep [12]. This downscaling of synaptic activity prevents the saturation of synaptic networks. On the other hand selective reactivations of cortical areas take place during post learning sleep, leading to an increase of markers for synaptic potentiation in these areas [13], [14]. Memory consolidation does not only involve changes in plasticity and connectivity of local neural networks, but also comprises systems level reorganization. During sleep newly encoded memories from the hippocampus are integrated for long term storage into the neocortex [15]. It is suggested that this information transfer is controlled by a precisely timed sequence of characteristic oscillation patterns during NREM sleep.

Another possible function of sleep is to facilitate the clearance of metabolic waste products from the brain. The brain gets cleared of its metabolic waste products by cerebrospinal fluid (CSF) flowing through its interstitial space. During sleep interstitial space in the brain increases, leading to an increase in CSF flow, resulting in an increased clearance rate of a radiolabeled metabolite from the brain [16].

Sleep is actively regulated in all animals with a nervous system that have been carefully studied [17]. Two distinct processes that regulate sleep have been identified, a circadian and a homeostatic.

\subsubsection{Circadian regulation of sleep}

Circadian regulation modulates the propensity, the "drive" for sleep over the $24 \mathrm{~h}$ cycle (Figure 1.1). The understanding of the circadian clock advanced largely by studies in simple organisms, as D. melanogaster and N. crassa. Circadian rhythmicity is generated by the activity of the suprachiasmatic nucleus (SCN). At the molecular base of the circadian clock are positive and negative autoregulatory feedback loops. The circadian clock gets mainly entrained by the light-dark cycle, but lifestyle and melatonin levels also have an influence [18]. 


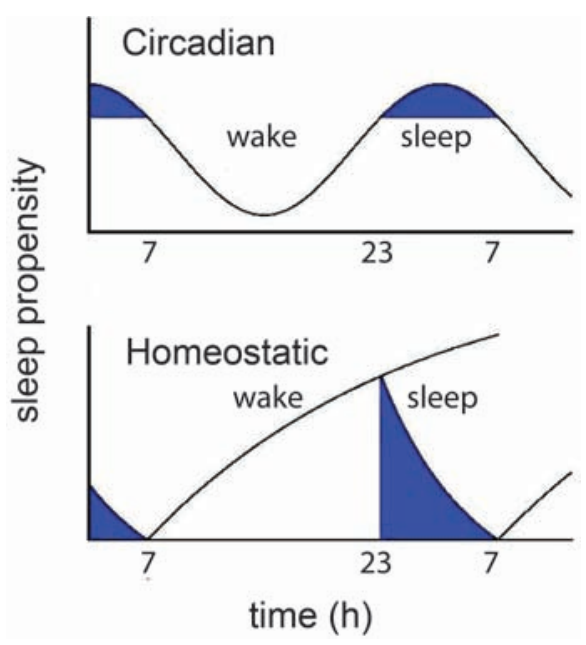

Figure 1.1: Sleep regulation. Shown is a scheme of circadian and homeostatic sleep regulation. Circadian regulation modulates the propensity, the "drive" for sleep over the $24 \mathrm{~h}$ cycle. Homeostatic regulation modulates sleep propensity as a function of prior sleep and wake time. Modified from http: //www.pharma.uzh.ch/research/chronobiology/ areas/sleepbiology/sleepeeg/Fig2.jpg

\subsubsection{Homeostatic regulation of sleep}

Homeostasis describes regulatory mechanisms that maintain the constancy of the physiology of an organism. Homeostatic regulation modulates sleep propensity as a function of prior sleep and wake time (Figure 1.1). The homeostatic pressure driving sleep increases during wake and decreases during sleep [19]. Sleep homeostasis regulates both sleep duration and intensity. As reaction to sleep deprivation (SD) homeostatic regulation leads to a sleep rebound. Both duration and intensity of previous wakefulness influence duration and intensity of consecutive sleep. Both REM and NREM sleep are known to be under homeostatic control.

EEG recordings of brain wave activity allow classifying the intensity of sleep and the propensity of sleep during wakefulness. Increased slow wave activity during sleep and increased high wave activity during wake correspond to high intensities in sleep and a high propensity of sleep during wakefulness respectively [20]. Interestingly prolonged wakefulness only leads to increased consecutive sleep, if it is accompanied by an increases of high frequency wave activity [21].

Accordingly, sleep deprivation induces an increase of high wave activity during wake and of slow wave activity during recovery sleep. While scoring for homeostatic effects of sleep deprivation, one has to actively control for stress induced side effects. Sleep deprivation has a strong effect on homeostatic regulation of sleep but only minor effects on circadian regulation, as shown in rats [22]. Nevertheless, homeostatic and circadian regulation seems to be closely linked to each other. Many mutations concerning the cir- 
cadian clock also induce altered homeostatic responses to sleep deprivation (reviewed in [23]).

On the neuronal level the regulation between sleep and waking relies on reciprocal interactions of sleep promoting neurons with wake promoting neurons in the hypothalamus and brainstem. Sleep promoting neurons are located in the ventrolateral preoptic nucleus (VLPO) and in the median preoptic area (MnPn) [24], [25]. Sleep active neurons are mostly silent and get specifically activated at sleep onset [26]. There is evidence indicating that they promote sleep by inhibiting wake promoting neurons via GABA and galanin signaling [27]. Neuronal signaling differs between wake and sleep. The secretion of neurotransmitters decreases during NREM and REM sleep, with the exception of acetylcholine, that returns to waking levels during REM sleep. Narcolepsy, a malfunction of sleep homeostasis, led to the discovery of the wake promoting role of the neurotransmitter hypocretin, also called orexin. Although EEG patterns of REM sleep and waking are very similar there are REM on cells and REM off cells.

The molecular basis of the sleep homeostat is less well understood. A working model for the sleep homeostat assumes that accumulation of a substance in the brain during wakefulness increases the propensity for sleep. Therefore scientists were screening for putative regulatory factors exhibiting increased concentrations in wake and decreased concentrations during sleep. Molecules related to energy metabolism, immune defense and neural plasticity were found that regulate sleep and waking. Out of this selection one molecule, adenosine, might relate energy shortage to an increased sleep pressure [28]. It is suggested that an increase of adenosine concentration in the basal forebrain inhibits neuronal activity of wake promoting neurons and thereby induces sleep [29]. As a second mechanism adenosine possibly activates sleep promoting neurons [30]. How wakefulness translates to an increase in adenosine concentration in selected brain areas is not understood. There is first evidence indicating that cholinergic cells in the basal forebrain trigger local adenosine production [31]. The pro inflammatory cytokine TNFalpha and the hormone prostaglandin 2, that independently have been associated with the sleep homeostat, have turned out to be upstream regulators of adenosine [8]. Similar to adenosine, an increase in nitric oxide concentration leads to increased recovery sleep. BDNF-1, a molecule involved in the process of synaptic potentiation, has been associated with sleep propensity. BDNF-1 concentration increases while waking and artificial induction of BDNF-1 increases sleep intensity [32].

\subsubsection{Conservation of sleep}

Using behavioral criteria, sleep has been identified in all intensively studied animals [17]. During evolution animals developed a multitude of different mechanisms to ensure sleep in varying conditions. Sleep can be monophasic or polyphasic, consisting of either one or multiple sleeping bouts per day. Sleep amount and timing vary strongly between species. Bats sleep up to 20 hours per day, whereas elephants and giraffes only about 4 hours per day. There is unihemispheric sleep in some marine mammals, like dolphins. EEG signatures are linked to the structure and function of the neocortex and 
vary largely between species. Reptiles and amphibians have higher amplitude brain activity during wakefulness than in sleep. Even within mammals, REM and NREM sleep vary a lot between different species, for example regarding sleep depth. However, despite this variety of sleep realizations, there is growing evidence for a fundamental conservation of sleep mechanisms across species.

In the 1930s studies on monozygotic and dizygotic twins were the foundation of a genetic basis for sleep. Since then hundred of genes that change their expression levels in a sleep wake dependent way have been found. These are highly conserved between species and have been associated with a couple of molecular pathways that are thought to regulate sleep and wakefulness. This suggests that functional and regulatory elements might be conserved between species too. Energy metabolism, cellular stress and synaptic potentiation have been related to higher transcription during wake. Whereas protein synthesis, synaptic depotentiation and membrane trafficking have higher transcription during sleep. Wake increased transcripts could serve other function than wake-sleep related. Moreover circadian regulation, neurotransmission, and other signaling pathways and ion channels are also conserved in sleep regulation. The following neurotransmission pathways have been found to influence sleep: noradrenergic, histaminergic, serotonergic, cholinergic, GABAergic, and hyptocretin/orexin system. It has been shown that manipulating ion channel activities leads to strong changes in sleep intensity and duration, as discussed in the review [33]. This is probably due to altered neuronal depolarization inducing altered excitability.

\subsection{C. elegans}

C. elegans is a nematode that belongs to the clade of ecdysozoa, which is characterized by a protective cuticle covering them. It lives in the soil or at the soil-air interface and feeds on bacteria that develop on rotten organic matter. It develops from egg to adult in about three days. Under favorable conditions it lives about two to three weeks and grows to a maximum length of about $2 \mathrm{~mm}$. C. elegans is a eutelic organism, having a fixed number of somatic cells. It possesses six pairs of chromosomes, five pairs of autosomes and one pair of sex chromosomes. Two sexes exist in C. elegans, hermaphrodites and males. Research is mostly conducted on hermaphrodites, whereas males are almost exclusively used to study their mating system.

C. elegans offers several advantages compared to mammals to investigate on sleep. It has a simple, well described nervous system, a fast lifecycle and little genetic redundancy. The nervous system of the hermaphrodite nematode consists of 302 neurons. The male has 79 additional neurons, that are mainly required for mating. It is unique for $C$. elegans, that the connectome - the neuronal connectivity of the entire nervous system - is known. It has about 6400 chemical synapses, 900 gap junctions and 1500 neuromuscular junctions. There is less genetic redundancy compared to mammals or even drosophila. This implies that inactivation of a gene more likely has an effect on the phenotype. For $C$. elegans there exists two strain librarys that cover $\mathrm{KO}$ mutations for a large portion of the C. elegans genes [34], [35]. The genomic sequence of C. elegans was 
the first to be deciphered. Therefore there are a multitude of genetic methods available, among others RNAi, optogenetics and genetically encoded calcium indicators. Most major mammalian neurochemistry is conserved in C. elegans, it has the neurotransmitters acetylcholine, glutamate, dopamine, serotonin, and GABA. However there is no noradrenaline or histamine but instead tyramine and octopamine. The major difference to mammals is that $C$. elegans has a different neuroanatomy. It does not have a cortex and therefore, brain wave patterns cannot be used to analyze sleep.

\subsubsection{C. elegans sleep}

The nematode C. elegans evolves through four larval stages (L1-L4) before reaching adulthood (Figure 1.2). At the end of each larval stage the worm molts, shedding its old cuticle that gets replaced by a newly synthesized one. Each molt is preceded by a quiescence phase, called lethargus, that lasts between two and three hours. It has been shown that lethargus fulfills all behavioral sleep criteria [36]. During lethargus, the worm exhibits prolonged bouts of quiescence [37], [36], [38]. It has an increased arousal threshold [36], [39] and quiescence is reversible by sensory stimulation [36], [40], [41]. It was shown that it has a sleep specific posture, including body wall muscle relaxation [38], [42]. Sleep deprivation leads to homeostatic sleep rebound (Raizen, [41]). It has been shown that sleep has an effect on the nervous system. During sleep basal and arousal induced sensory neuron activity is reduced [39]. Recently a sleep active, sleep promoting neuron has been discovered in C. elegans [40].

Quiescence is mainly restricted to larval development. Adult quiescence occurs for short times that last from seconds to a few minutes. It is currently unclear if adult quiescence is as well a sleep state. It was shown that satiety, heat stress, toxic chemicals and a few other factors have an impact on adult quiescence. In C. elegans the circadian period ortholog lin-42 controls the timing of larval development [43], [44]. Analogous to mammals, rhymiticity is induced by oscillations of mRNA and protein levels. Circadian rhythmicity exists to a weaker extent in adult animals, controlling behavior, metabolic processes and mRNA expression [45], [46], [47]. This rhythmicity seems not to be regulated by Lin-42 [47]. Sleep active and wake active genes have been identified by transcriptional expression profiling [40]. Pathways that have been associated with sleep regulation in other organisms, like cGMP/cAMP, adenosine, notch signaling and growth factors are mostly conserved in C. elegans [48]. egl-4, a cGMP-dependent protein kinase and pka, a cAMP-dependent protein kinase promote quiescence [36]. Also the notch signaling co-ligand osm-11 promotes sleep [49]. Similarly, EGF, an epidermal growth factor implicated in stress response, induces quiescence if overexpressed. This is mediated by the ALA neuron and flp-13, FMRFamide-like neuropeptide signaling [50]. Another neuropeptide, $n l p-22$ regulates sleep in C. elegans, as shown by overexpression that leads to increased quiescence [51]. nlp-22 mRNA expression is cyclic following the sleep cycle that is regulated by lin-42. Neuropeptides are known to regulate sleep in mammals, too. 


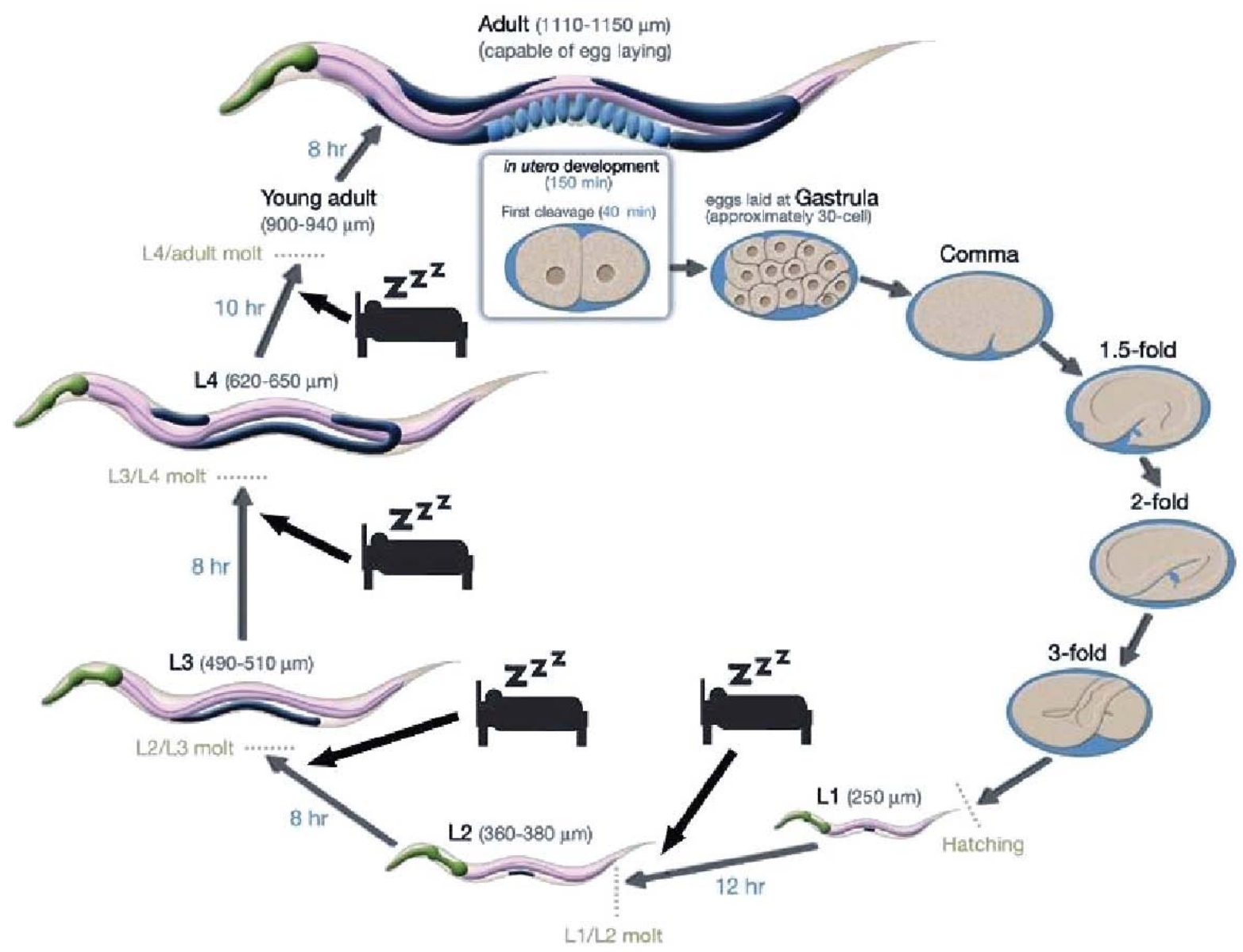

Figure 1.2: The lifecycle of C. elegans. C. elegans evolves through four larval stages (L1L4) before reaching adulthood. At the end of each larval stage the worm molts, shedding its old cuticle that gets replaced by a newly synthesized one. Each molt is preceded by a quiescence phase, called lethargus, that lasts between two and three hours. The development from egg to adult lasts about three days. Modified from http://www.wormatlas.org/verl/ handbook/fig.s/IntrofIG6.jpg

\subsubsection{Sleep homeostasis in C. elegans}

It has been shown that sleep following a phase of sleep deprivation is deeper, i.e., the response latency to external stimuli is prolonged. Following sleep deprivation avoidance behavior upon administration of octanol is delayed [36]. The response latency correlates with prior sleep deprivation length, as predicted for a homeostatic regulation. Mean and peak quiescence bout duration increases following sleep deprivation [36]. But duration of sleep timing is not prolonged [36]. It is reported that continous sleep deprivation leads to death [41]. Mutants of daf-16, a foxo transcription factor, do not 
Chapter 1. Introduction

show a homeostatic response to sleep deprivation. Restoring daf-16 expression in muscle, but not in neurons, rescues the homeostatic sleep phenotype [41]. 


\subsection{Thesis Aims}

The aim of this thesis is to gain a better understanding of the sleep homeostat of $C$. elegans. Hereby I will focus on the neuronal and molecular mechanisms underlying the sleep homeostat. In particular I have the following objectives:

- To develop an automated sleep deprivation setup allowing simultaneous behavioral analysis and calcium imaging of neurons and to apply it to score for homeostatic effects.

- To investigate on neuronal activity underlying the sleep homeostat.

- To investigate sleep homeostasis in sensory neurons.

- To investigate the neurotransmitter/neuropeptide signaling underlying the sleep homeostat.

- To investigate sleep homeostasis using sleep mutants. 



\section{Chapter 2}

\section{Methods}

\subsection{C. elegans maintenance}

C. elegans was maintained on nematode growth medium (NGM) plates seeded with $E$. coli OP50 as previously described [52]. Worms were grown at temperatures between $15^{\circ} \mathrm{C}$ and $25^{\circ} \mathrm{C}$. Experiments were conducted at $21.5^{\circ} \mathrm{C}$. Most worm handling relied on using a homemade thin platinum wire pick, as described in [53].

\subsection{Creating transgenic $C$. elegans strains}

\subsubsection{Plasmid construction by Gateway Cloning TM}

All constructs were cloned using the Multisite Gateway ${ }^{\mathrm{TM}}$ system from Invitrogen [54]. Using this system three individual donor plasmids are combined to a final construct plasmid. The donor plasmids I used in this thesis to generate new constructs were synthesized by a commercial supplier. All constructs obtained from Gateway LR TM reactions were sequenced for verification. Successful cloning was confirmed by comparing the Sanger sequencing results with the expected sequence using megablast sequence alignment [55]. The complete list of constructs I created are listed in appendix B.2.

\subsubsection{Microinjection and microparticle bombardment}

Transformation of a DNA plasmid construct into C. elegans can be achieved either by microinjection or microparticle bombardment [56], [57]. The latter often induces integration of the DNA construct onto a chromosome [58]. Chromosomal integration of a DNA construct leads to higher expression stability and less germline silencing as compared to extrachromosomal arrays. Most strains described in this thesis were generated using microparticle bombardment as described in [57]. I bombarded unc-119(ed3) mutants, which show an uncoordinated phenotype. The DNA construct carried a unc-119rescue as a selection marker. Most transgenic strains were generated using microparticle bombardment. Microinjection was only used to transform some of the lim- 6 rescue constructs. The complete list of strains that I created and/or used for experiments during this thesis can be found in appendix B.1. 


\subsubsection{Crossings of $C$. elegans strains}

Strains were crossed using standard procedures [52]. For all strains that did not carry a fluorescent marker, the presence of the genotype was verified after crossing by PCR genotyping.

\subsubsection{PCR genotyping}

PCR genotyping was done by duplex PCR genotyping as described [59]. It is based on the design of three primers, producing two different-sized products, one only arising from the wild-type allele, the other only arising from the mutant allele. In a first step, DNA is obtained by single worm-lysis [60]. Then the primers are added and a duplex PCR is performed. In the last step agarose gel electrophoresis is conducted visualizing the PCR products from which the genotype of the animals can be deduced. The complete list of primers that I created can be found in appendix B.3.

\subsection{Calcium imaging and optogenetics}

\subsubsection{In vivo calcium imaging}

Traditionally neuronal activity has been measured using electrophysiology. In C. elegans electrophysiology measurements are difficult to perform because of its hydrostatic skeleton and its small size. Instead genetically encoded calcium indicators (GECIs) are used to image neuronal activity [61]. These indicators increase their fluorescence if calcium is present. In C. elegans a multitude of promoters are known allowing expression in subsets of neurons, covering all neurons. A widely used genetic calcium indicator is GCaMP, a fusion of circularly permutated green fluorescent protein (GFP), calmodulin, and M13, a peptide sequence from myosin light chain kinase [62], [63]. Without calcium, the circular GFP structure leads to quenching. Upon calcium binding, calmodulin undergoes a conformational change, allowing M13 to bind thereby changing the circular shape of the GFP and reducing the quenching. GCaMP has faster kinetics, a higher baseline fluorescence, and a larger dynamic range than ratiometric indicators like Chameleon [64]. To perform quasi ratiometric measurements a reference fluorescence protein, whose fluorescence does not change with cellular calcium concentration, can be co-expressed with GCaMP. Importantly this reference fluorescence protein must have an excitation and emission spectrum that is distinct from that of GCaMP. Most calcium imaging in this thesis is done using GCaMP3.35 that is co-expressed with a codon optimized version of the far red fluorescent protein mKate2 [65], [66]. GCaMP3.35 corresponds to GCaMP3 minus the first 35 aminoacids, and a codon optimization as described in [39]. RIS activity measurements using the unc-47 promoter were performed with GCaMP3 [63]. RIA activity measurements were performed using GCaMP3.3, a variant of GCaMP3 [67]. 


\subsubsection{Optogenetics}

Optogenetics allow the precise temporal and spatial activation and inhibition of neurons with light. The breakthrough for optogenetics came in 2005 when several research groups independently showed that channelrhodopsin-2(ChR2) expressed in neurons is functional [68], [69]. ChR2 is a light gated cation channel that has been genetically isolated from the algae Chlamydomona reinhardtii [70], [71]. It has a low rate of inactivation, a fast rise time $(<200 \mathrm{~ms})$ and a quick recovery time. It is a blue light activatable channel, having its action spectrum peak at about $460 \mathrm{~nm}$ [71]. ChR2 activation requires the cofactor all-trans-Retinal, that is endogenously present in mammals, but not in $C$. elegans. The standard way to introduce Retinal in C. elegans is by adding it to its bacterial food. Most channelrhodopsin experiments in this thesis are done using a codon optimized version of ChR2(H134R), a mutation of ChR2 yielding increased photocurrents. Red shifted variants of channelrhodopsin have been developed recently. Especially promising according to the literature were the following two: C1V1 a chimer of channelrhodopsin-1 (ChR1) and Chrimson, a red shifted channelrhodopsin, that came out from sequencing of 127 algae transcriptomes [72]. Of future interest might be another recently engineered red shifted ChR variant ReaChR [73].

Additionally to the beforehand presented rhodopsins there exist others that can be used to hyperpolarise neurons. These are most notably halorhodopsin (chloride channel) and archaerhodopsin (proton pump) [74] , [75]. The latter yielding a more complete inactivation and having a faster recovery period [75].

\subsection{Imaging setup}

\subsubsection{Microfluidic device}

For most experiments $C$. elegans have been cultured in agarose hydrogel microcompartments as described [76]. Briefly, eggs were placed together with bacterial food (E. coli OP50) into agarose microcompartments that were cast using a PDMS mold.

\subsubsection{Differential interference contrast (DIC) and brightfield imaging}

For behavioral analysis worms were imaged inside agarose microcompartments using DIC or brightfield. As transmitted light source a $100 \mathrm{~W}$ Halogen lamp was used that was filtered through a standard infrared filter (Chroma) resulting in infrared light. Respectively a $20 \times$ CFI Planapochromat VC Objective or a $40 \times$ CFI S Fluor Oil Objective (both Nikon) were used. Most DIC/brightfield imaging was done using an Andor Luca camera. For a few experiments an Andor iXon EMCCD camera or an Andor Neo sCMOS camera were used. Two different protocols were used for imaging. Either continuous time lapse imaging ( $1 \mathrm{~s}$ to $5 \mathrm{~s}$ interval) or burst mode imaging every $10 \mathrm{~min}$ to $15 \mathrm{~min}$ ( 40 frames with $0.5 \mathrm{~s}$ interval). For sleep deprivation experiments only time lapse imaging with permanent illumination was used. 


\subsubsection{Fluorescence imaging}

Fluorescence imaging was performed essentially as previously described [39], [40]. Experiments were performed using an Andor iXon EMCCD camera and LED illumination (CoolLED) with standard GFP and Texas Red filter sets (Chroma). Exposure time was set to $5 \mathrm{~ms}$ and allowed imaging of moving worms without blurring. The EM gain of the camera was set between 100 and 250. The LED was triggered using the TTL fire signal of the EMCCD camera to illuminate only during exposure. LED intensity was in the range of $1 \mathrm{~mW} \mathrm{~mm}^{-2}$ to $5 \mathrm{~mW} \mathrm{~mm}^{-2}$. The illumination did not cause bleaching or any detectable behavioral changes. Most calcium imaging experiments were performed in agarose hydrogel microcompartments. The only exception are the measurements of neuronal activity following ChR2 activation of aptf-1 expressing neurons (section 3.7.3) that have been done by fixating the worm on a $2 \%$ agarose pad using Levamisole. Calcium imaging experiments were recorded either with a $20 \times$ CFI Planapochromat VC Objective or a $40 \times$ CFI S Fluor Oil Objective. Stacks of 16-35 frames each were taken using a "Nano Scan Z" (Prior Scientific). Z-resolution of the stacks was $1 \mu \mathrm{m}$. Stacks were either projected into one image using a maximum intensity projection or the slice of interest was manually selected. For graphical representation of expression patterns the maximum intensity projection was used. For calcium imaging experiments the slice of interest was selected manually.

\subsubsection{Spinning disc imaging}

Spinning disc imaging was performed with an Andor Revolution spinning disc system using a $488 \mathrm{~nm}$ laser, a Yokogawa X1 spinning disc head, a $40 \times$ or $100 \times$ oil objective and an Andor iXon EMCCD camera.

\subsection{Experimental sleep deprivation setup}

\subsubsection{Microscope setup for simultaneous brightfield and fluorescence imaging}

The basis for the automated sleep deprivation setup is a Nikon eclipse Ti microscope. This microscope was equipped with an additional second layer and appropriate filters allowing simultaneous measurements with two cameras (Figure 2.1). The upper layer is used to connect a LED light source to the microscope setup, whereas the lower layer is used to split the DIC/brightfield image of the worm from the calcium imaging. This is achieved by using a dichroic filter that separates the infrared light used for DIC/brightfield imaging from the green and red fluorescence light. I disassembled the GFP filter cube and placed the excitation filter and dichroic filter in the upper layer. The emission filter I added to the filter block containing the infrared dichroic filter in the second layer, in the optical path used for calcium imaging.

For DIC/brightfield imaging I used an ANDOR Luca EMCCD camera and for calcium imaging an ANDOR iXon EMCCD-camera. Because of the different camera chip sizes 


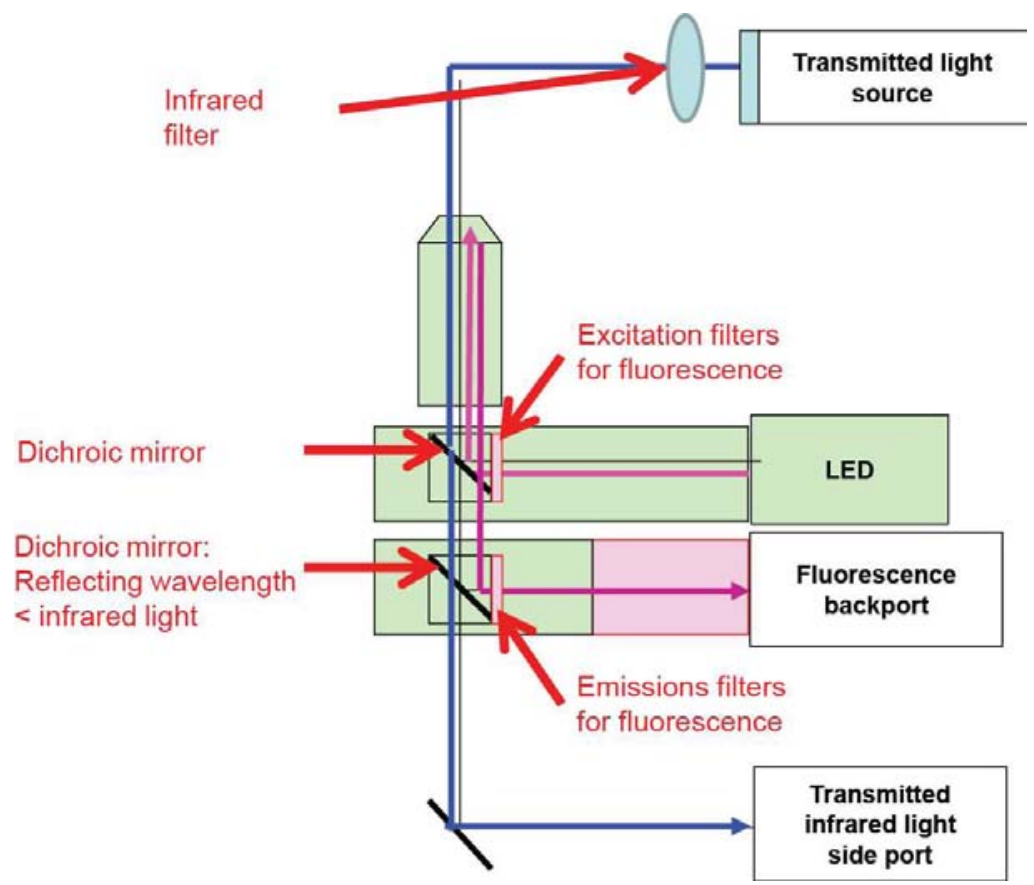

Figure 2.1: Microscope setup for simultaneous brightfield and fluorescence imaging.

I had to introduce a $0.45 \times$ magnification lens in the optical path of the ANDOR Luca camera. I decided to use two separate computers to control brightfield and calcium imaging. The brightfield imaging is controlled by computer 1 using LABVIEW, whereas the calcium imaging is controlled by computer 2 via Andor IQ software. Brigthfield imaging and calcium imaging can be synchronized by externally triggering either camera with a TTL signal.

\subsubsection{Automated sleep deprivation setup}

Sleep detection is based on custom written LABVIEW routines (see section 2.6). Sleep deprivation is triggered by LABVIEW using TTL outputs of the National Instruments PCIe-6509 data acquisition card. The data acquisition card is connected by a custom made adapter and BNC-cables to the external TTL trigger input of the deprivation device.

\subsubsection{Mechanical stimulation by dish tapping}

Mechanical touch has been intensively studied in C. elegans [77]. Manual mechanic stimulation with an eyelash has been used to sleep deprive C. elegans [36]. To automatize sleep deprivation I used dish-tapping instead. The dish-tapper I used consists of a custom made aluminum holder for a sample and a piston that gets accelerated by an electromagnet as described [39], [40]. I tried two different electromagnets vary- 


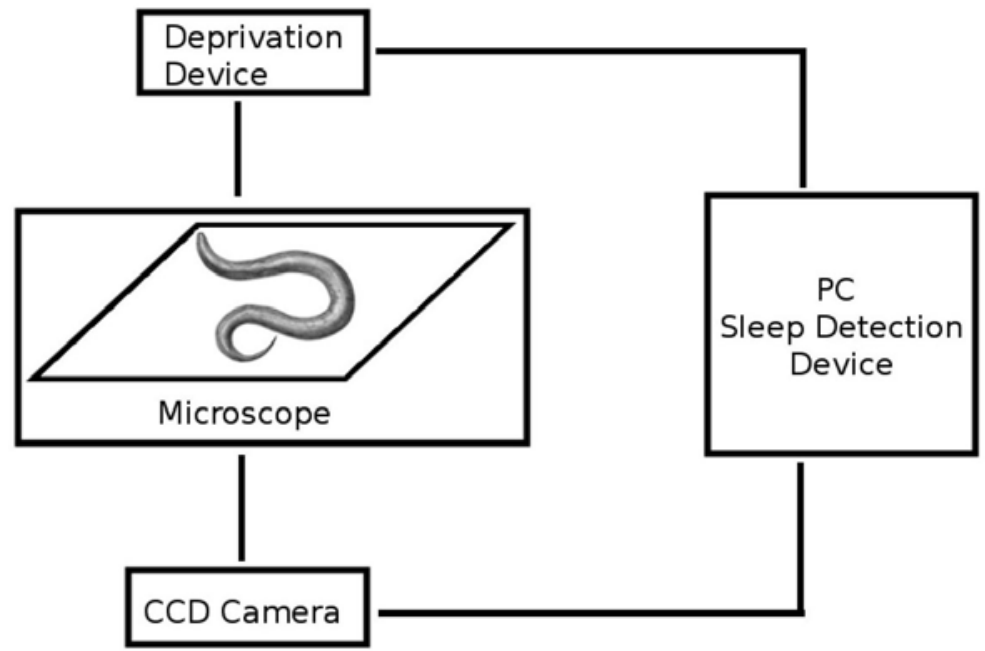

Figure 2.2: Scheme of the sleep deprivation setup. The worm is observed on a microscope using a CCD camera. Sleep is detected and a deprivation stimulus is triggered by custom written algorithms on a computer.

ing in power, the Kuhnke magnets H2286 (mild dish tapping) and H6286 (strong dish tapping). The tapping stimulus gets delivered horizontally in the plane of the sample, restricting sample movement mainly to the xy-plane. Image acquisition and dish tapping were shuttered in such a way that no apparent blurring due to tapping-induced movement occurred. The standard (strong) tapping stimulus for sleep deprivation experiments consisted of 5 taps that were administered in a 1s interval. The only exception to this protocol was for mild dish tapping, were a tapping stimulus consisted of a single tap.

\subsubsection{Channelrhodopsin experiments}

Most channelrhodopsin experiments were performed inside agarose microcompartments, as previously described [40]. The only exception being the aptf-1 ChR2 activation measurements in 3.7.3, that have been done by fixating the worm on a $2 \%$ agarose pad and immobilizing it using levamisol. Hermaphrodite mother worms were grown on medium that was supplemented with $0.2 \mathrm{mM}$ all-trans-Retinal (Sigma). Eggs from these mothers and food from the same plates were placed into microcompartments without any further Retinal supplementation. Worms were stimulated with a LED of $490 \mathrm{~nm}$ with $0.18 \mathrm{~mW} \mathrm{~mm}^{-2}$ to $0.39 \mathrm{~mW} \mathrm{~mm}^{-2}$ as measured with a light voltmeter. For the assessment which stimulation intensity is best suited for sleep deprivation also other intensities have been used, as it is indicated in the text. For excitation of red shifted channelrhodopsin worms were stimulated with an LED of $585 \mathrm{~nm}$ wavelength with $0.529 \mathrm{~mW} \mathrm{~mm}^{-2}$. 


\section{6 "C. elegans automated sleep deprivation"}

I wrote the "C. elegans automated sleep deprivation" program in LABVIEW. I chose LABVIEW for the following reasons:

- A preexisting integration of interfaces for many devices, like cameras and data acquisition cards

- A large library containing functions for data acquisition, mathematics, statistics, and most important an advanced graphical user interface (GUI)

- A graphical programming approach, that makes it easy for future users not having a background in programming to modify the program accordingly to their needs

LabVIEW functions/routines are called virtual instruments (VIs). Instead of a written source code each VI consists of a block diagram, a front panel and a connector panel. The block diagram is the graphical equivalent of the written source code. The front panel is a graphical user interface corresponding to the block diagram, that is automatically created by LABVIEW. The connector panel represents the input and output parameters of the VI that have to be used by another VI to call this VI. In the following paragraph I comment the source code of the LABVIEW "C. elegans automated sleep deprivation" program. The "C. elegans automated sleep deprivation" program is based on the call of 9 subroutines that are visualized in the scheme below (Figure 2.3). A

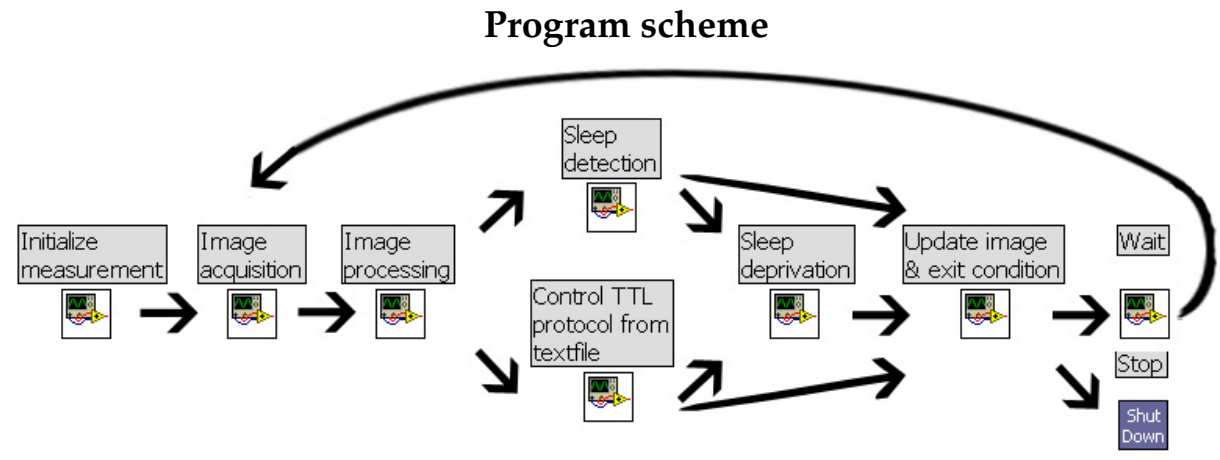

Figure 2.3: Program scheme

measurement always starts with the "Initialize measurement VI", that is followed by the "Image acquisition VI" and the "Image processing VI". Depending on whether sleep deprivation or control mode is chosen, either the "Sleep detection VI" or the "Control TTL protocol from textfile VI" is executed. In case of a successful sleep detection "Sleep deprivation VI" follows, or in case of no sleep detection the program continues directly with "Update image detection \& exit condition VI". Evaluation of the exit condition either runs the "Wait VI" or shuts down the measurement with the "ShutDown VI". The 
"Wait VI" then triggers again the "Image acquisition VI" leading to a cyclic VI execution. The LABVIEW block diagram implementation of this scheme is shown in Figure 2.4. Additionally to the scheme, a mode prior start measurement has been added to setup the image. If in the mode prior start measurement the program loops after the "Image acquisition VI" directly to the "Wait VI". 
2.6. "C. elegans automated sleep deprivation"

\section{Main}
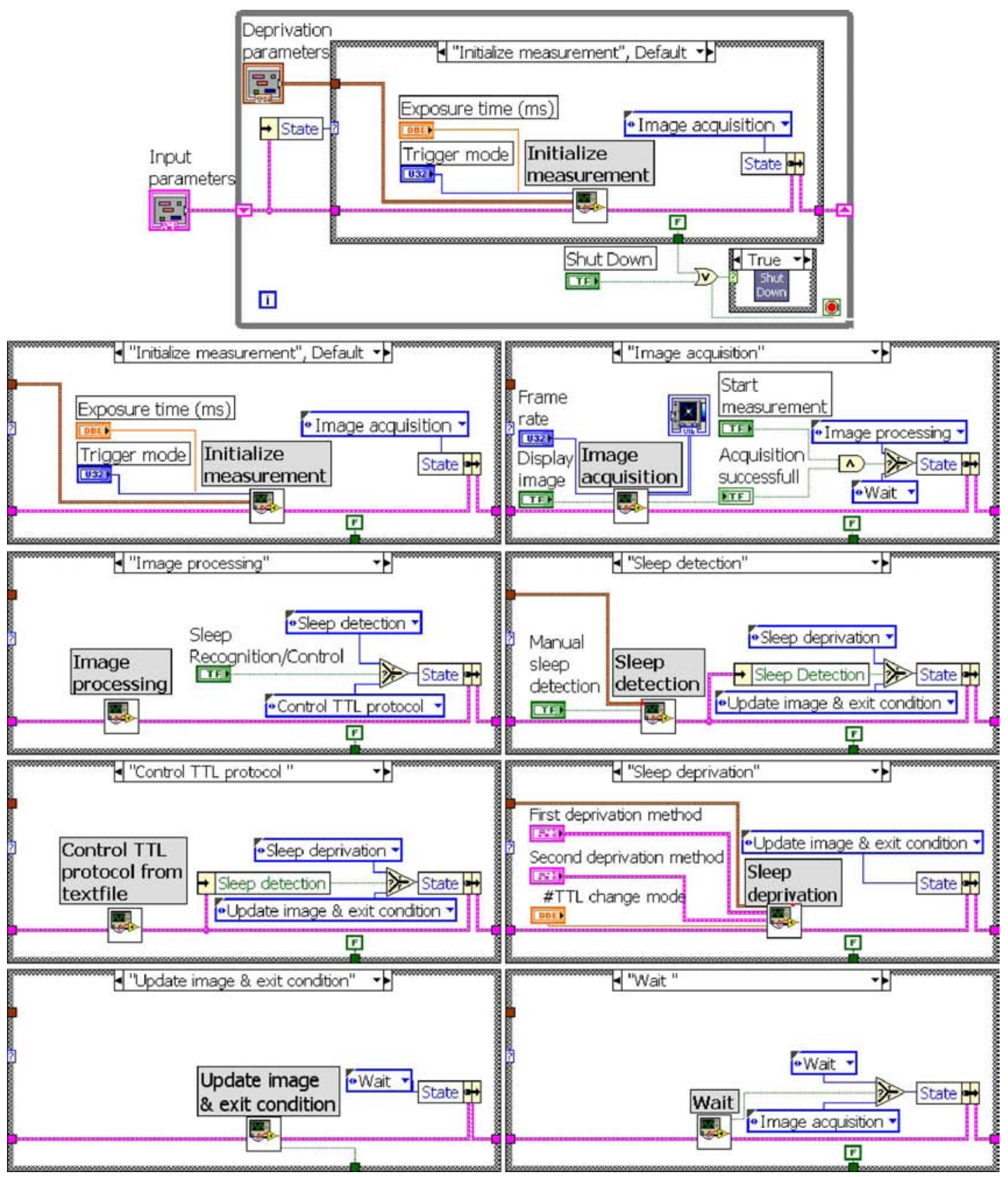

Figure 2.4: Maind1ALLIncl

\subsubsection{Initialize measurement}

The "Initialize measurement VI" (Figure 2.5) sets up the parameters used during the measurement ("Global and local image subtraction arrays VI", "Multiple animals pa- 
rameters VI", "Single animal subimage pixels VI"), creates the folders to save the measurement files ("Create folder VI") and initializes the camera settings ("Intialize camera settings VI"). The "Initialize measurement VI" is run only once at the beginning of a measurement.

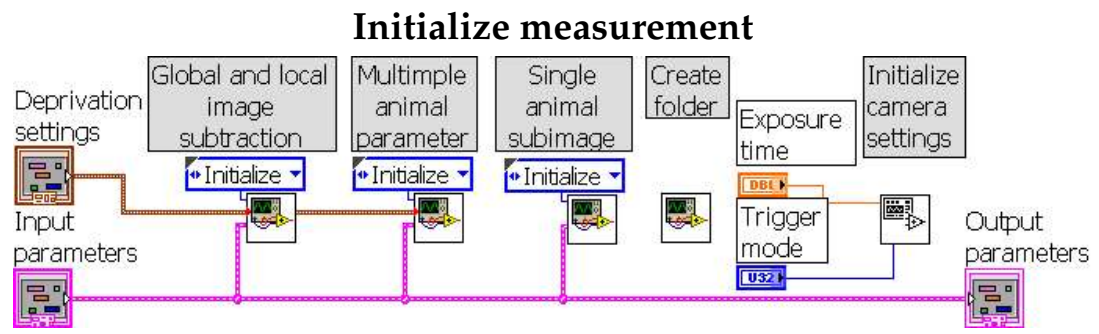

Figure 2.5: Initialize measurement

The "Global and local image subtraction array VI" (Figure 2.6) stores the global and local image subtraction values that are used to evaluate the sleep detection criterion. At this time point the "Global and local image subtraction array VI" is initialized. Setting up a one-dimensional array of length "\# Images for sleep detection" filled with zeros for the local image subtraction array. And a one-dimensional array with one zero element for the global image subtraction array. The "Multiple animal parameters VI" (Figure 2.7)

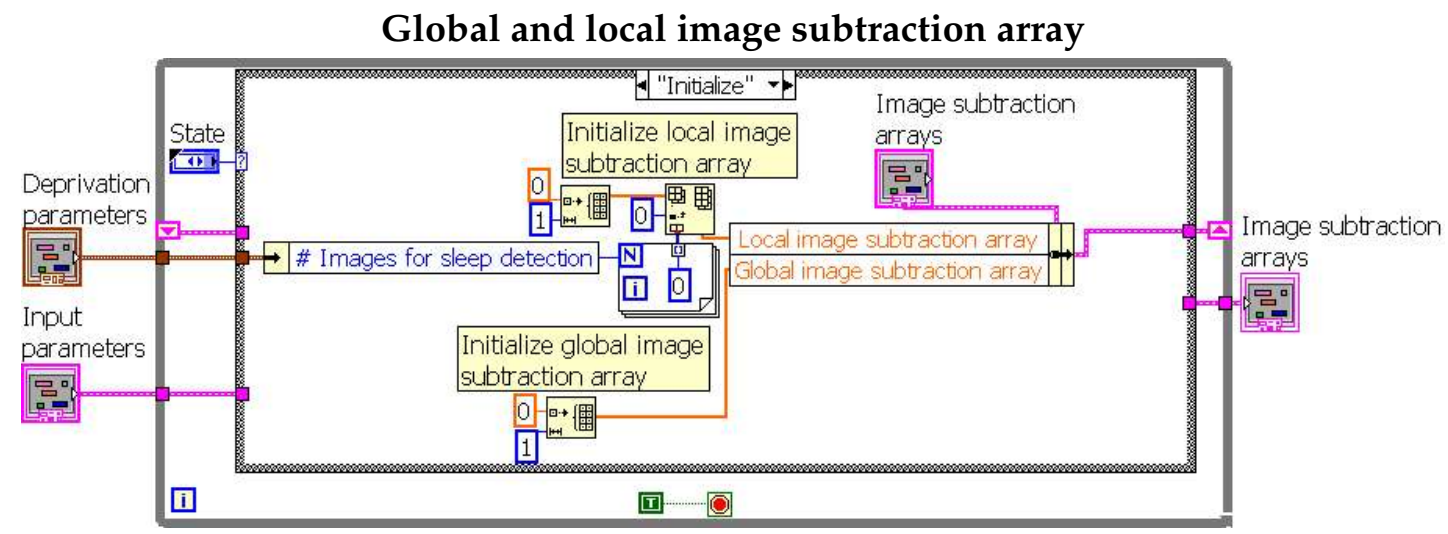

Figure 2.6: Global and local image subtraction array

calculates the index of the sleep deprivation animal and the total number of animals. The "Single animal subimage pixel ranges VI" (Figure 2.8) calculates from the total number of pixels of the camera, the pixel ranges corresponding to single animals. It does so by dividing the total image into equally sized subimages according to the \# animals per row/column.

The "Create folder VI" (Figure 2.9) verifies whether a folder with the same strain name and date already exists. If no folder exists it creates a new folder. If the folder already exists it gives an error message. In this case the user should rename or copy the pre- 
2.6. "C. elegans automated sleep deprivation"

Multiple animal parameters

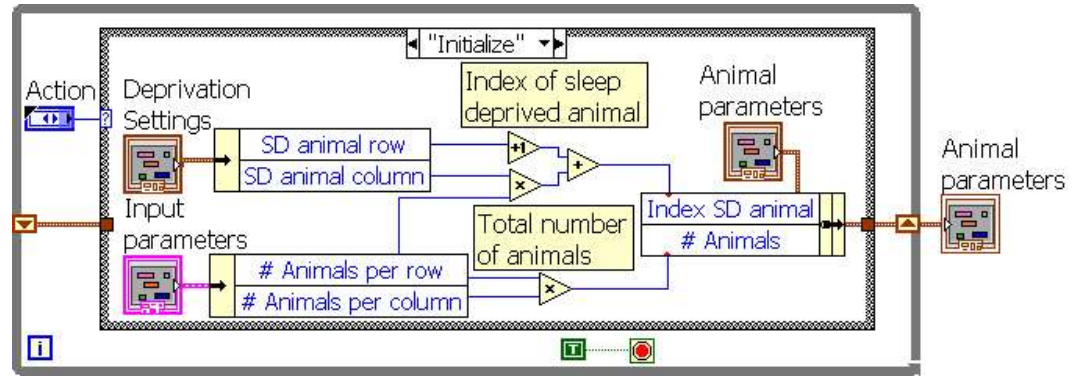

Figure 2.7: Multiple animal parameters

Single animal subimage pixel ranges

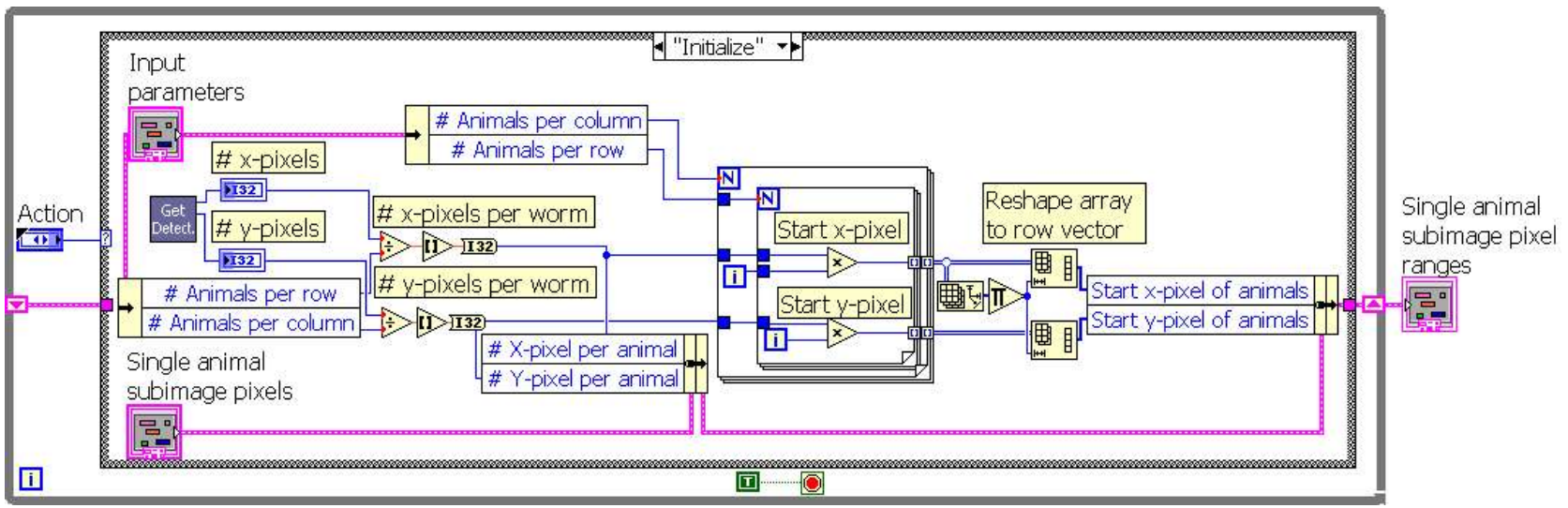

Figure 2.8: Single animal subimage pixel ranges

existing folder to another location. If the user continues the measurement despite an error message the old measurement data is overwritten.

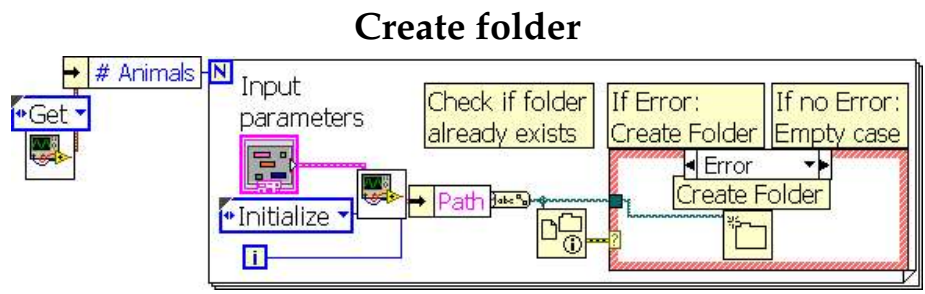

Figure 2.9: Create folder

The "Initialize camera settings VI" (Figure 2.10) starts the initialization of the camera and sets up the camera parameters used for the measurement. Of the parameters set here only the acquisition time and trigger mode should be varied using the main front 
panel.

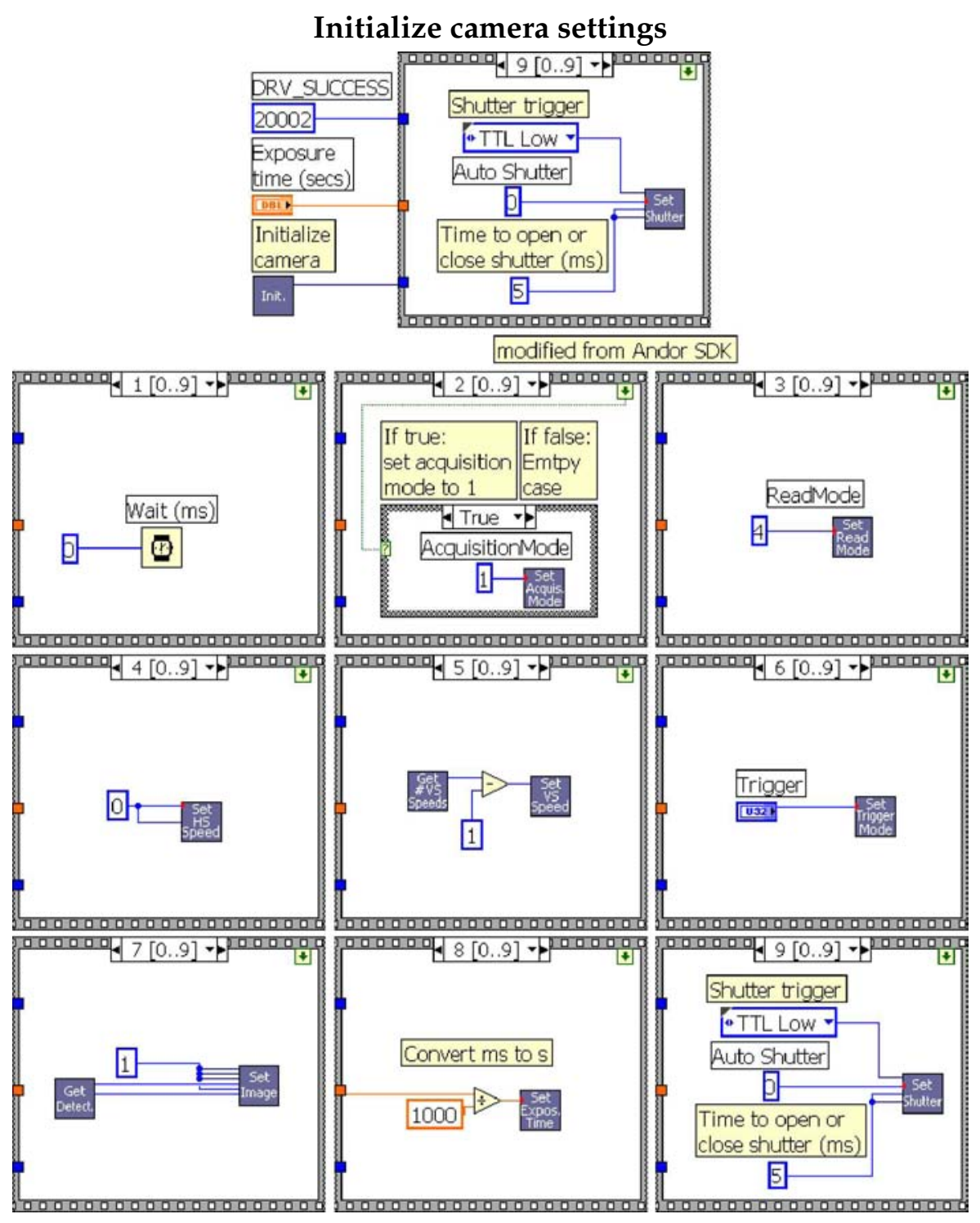

Figure 2.10: Initialize camera settings

\subsubsection{Image acquisition}

The "Image acquisition VI" (Figure 2.11) starts the image acquisition ("Start acquisition VI") and if the acquisition is successful it waits for the end of acquisition ("Wait for end of acquisition VI") and stores a converted version of it for display and further image processing ("Get image VI") . 


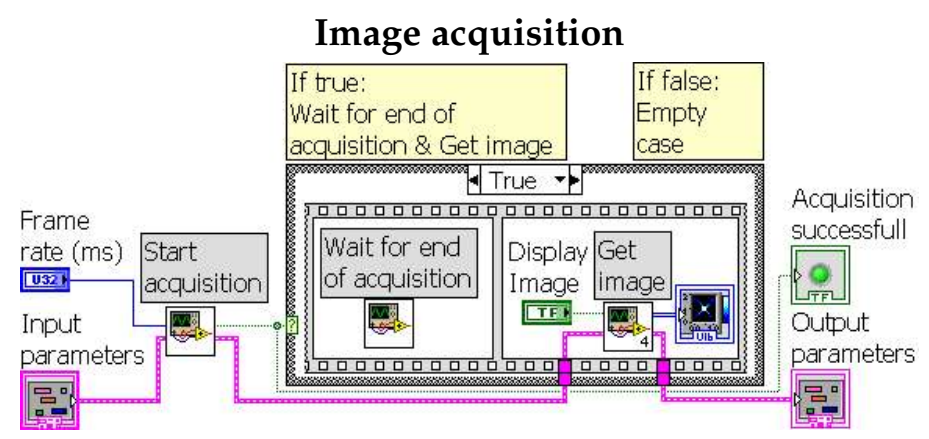

Figure 2.11: Image acquisition

The "Start acquisition VI" (Figure 2.12) adjusts the frame rate to account for delays due to the program execution, tries to start the acquisition and outputs the acquisition time and whether the acquisition was successfully started. The "Wait for end of acquisition

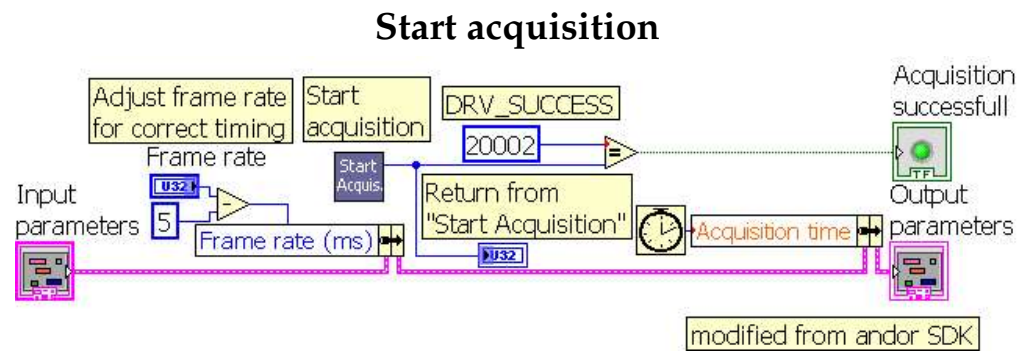

Figure 2.12: Start acquisition

VI" (Figure 2.13) verifies if the image acquisition has been completed. As long as this is not the case it introduces a $5 \mathrm{~ms}$ delay and then repeats the verification. The "Get image

\section{Finish acquisition}

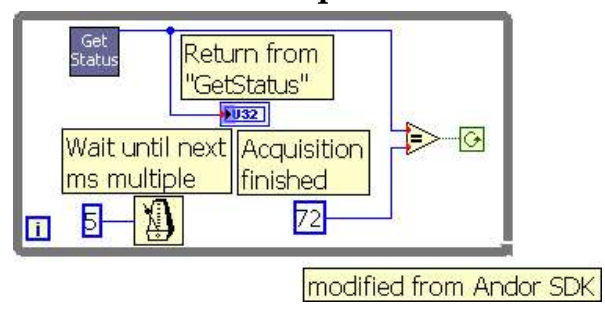

Figure 2.13: Finish acquisition

VI" (Figure 2.14) converts the acquired image that is stored in a one-dimensional array to a two-dimensional array, corresponding to the image dimensions. This converted image is stored for further image processing. If "display image" is chosen in the main front panel the image is displayed. 


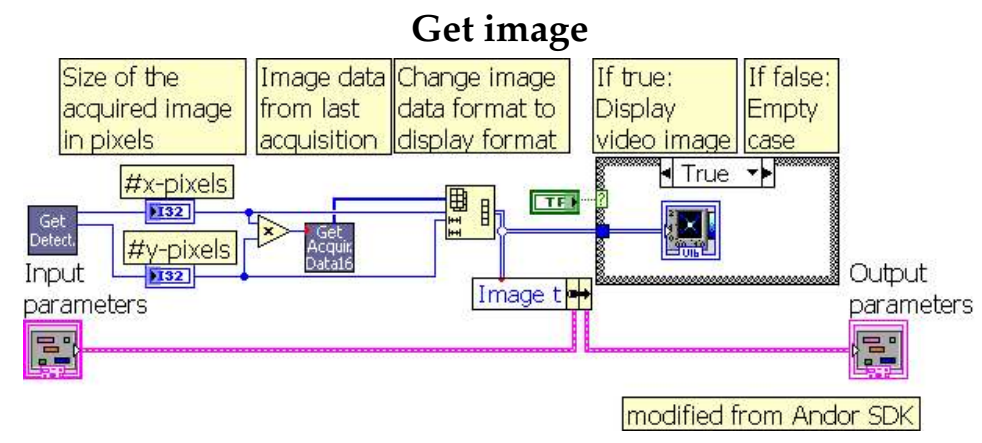

Figure 2.14: Get image

\subsubsection{Image processing}

The "Image processing VI" (Figure 2.15) saves single animal image subselections, calculates and saves corresponding image subtraction values and determines the image subtraction value of the sleep deprivation animal. For each animal it generates new file- and pathnames ("File-pathname VI"), creates the image subselection ("Create image subselection VI"), saves it as a tiff file ("Save array to image VI") and saves the image acquisition time to a textfile ("Save image acquisition time to textfile VI"). If images have been acquired before it calculates the image subselection of the previous image as well ("Create image subselection VI"), then calculates the image subtraction value ("Calculate image subtraction value VI"), saves it to a text file ("Save image subtraction value VI") and determines and outputs the image subtraction value of the sleep deprivation animal ("Select image subtraction value of SD animal VI").

\section{Image processing}

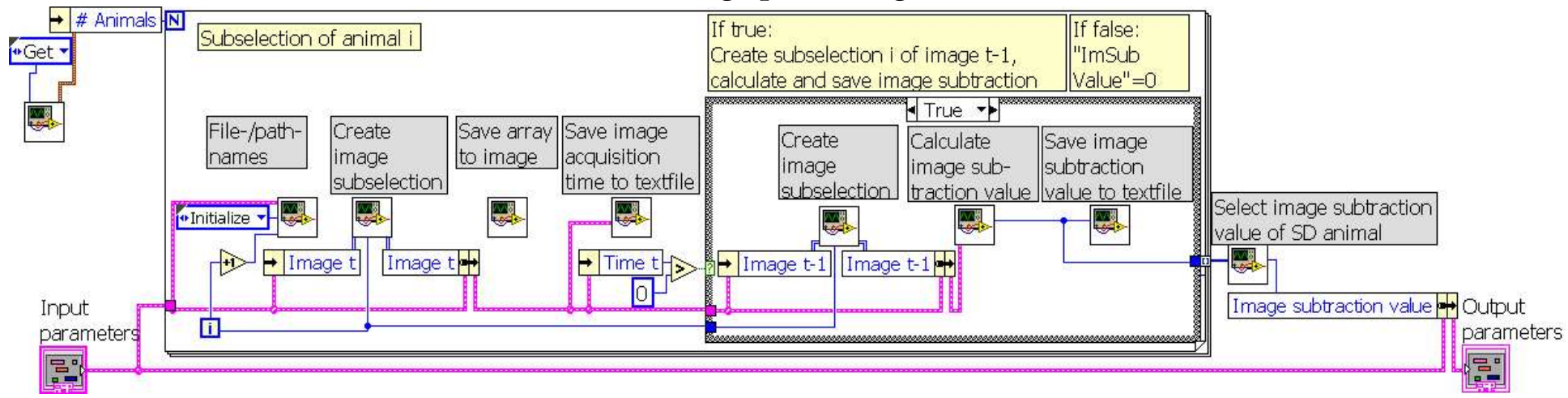

Figure 2.15: Image processing

The "File-pathname VI" (Figure 2.16) generates and outputs new file- and pathnames for the animal with the index $i$. Calling it with the initialization option initializes the path, the filestem, and the filestem including the current time $t$ and outputs them. The 
filestem denominates the filename preceded by the path, but without file extension.

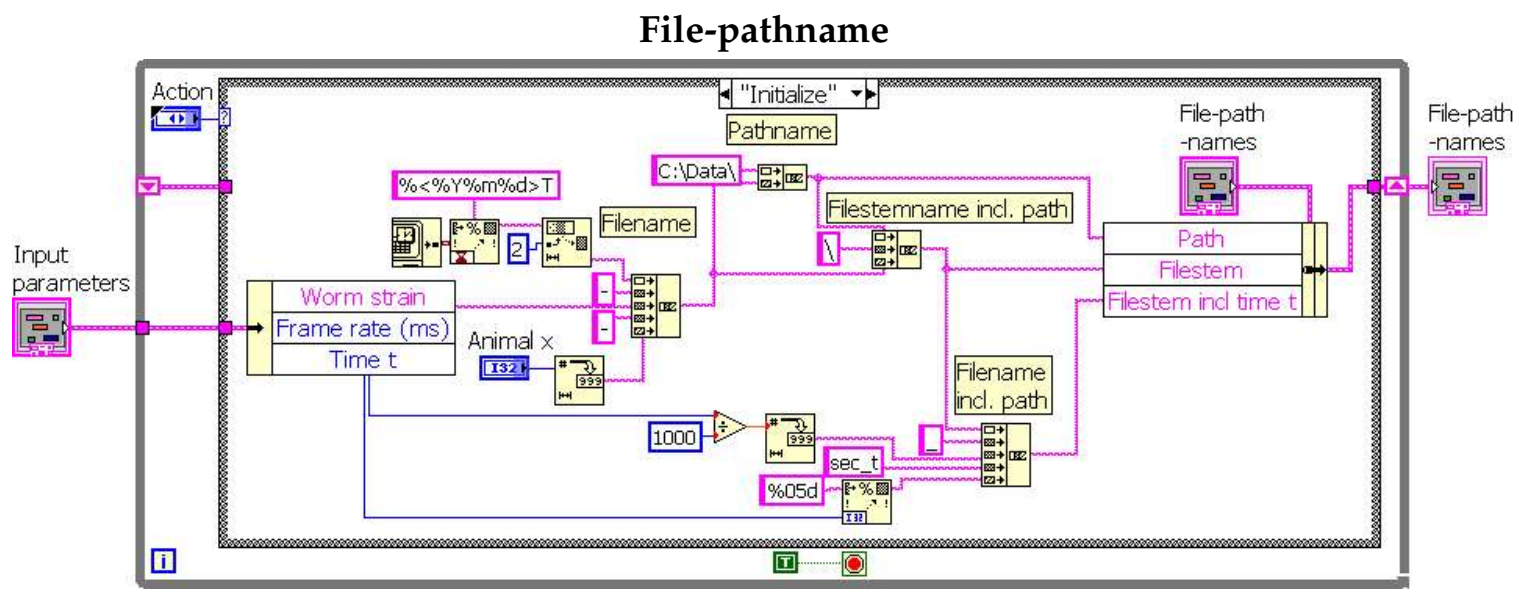

Figure 2.16: File-pathname

The "Create image subselection VI" (Figure 2.17) determines and outputs the image subselection of the full image that corresponds to the single animal with index $i$.

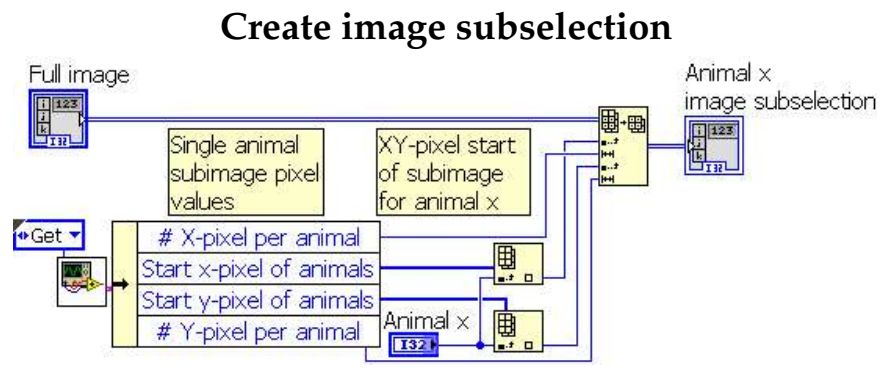

Figure 2.17: Create image subselection

The "Save array to image VI" (Figure 2.18) saves the subselection image array as a 16 bit grayscale tiff file. To output the image array as a tiff file the following routines that are included in the LABVIEW IMAQ Vision are used: "IMAQ Create", "IMAQ ArrayToImage", "IMAQ GetPalette" and "IMAQ WriteFile". The "Save image acquisition time to textfile VI" (Figure 2.19) saves the image acquisition time to the textfile named "Strain-Name-AnimalIndex-Date-time-loc.txt".

The "Calculate image subtraction value VI" (Figure 2.20) calculates the image subtraction value, outputs and stores it.

The "Save image subtraction value VI" (Figure 2.21) saves the image subtraction value to the textfile named "Strain-Name-AnimalIndex-Date-subtraction.txt"

The "Select image subtraction value of SD animal VI" (Figure 2.22) determines the image subtraction value of the sleep deprivation animal and outputs it. 


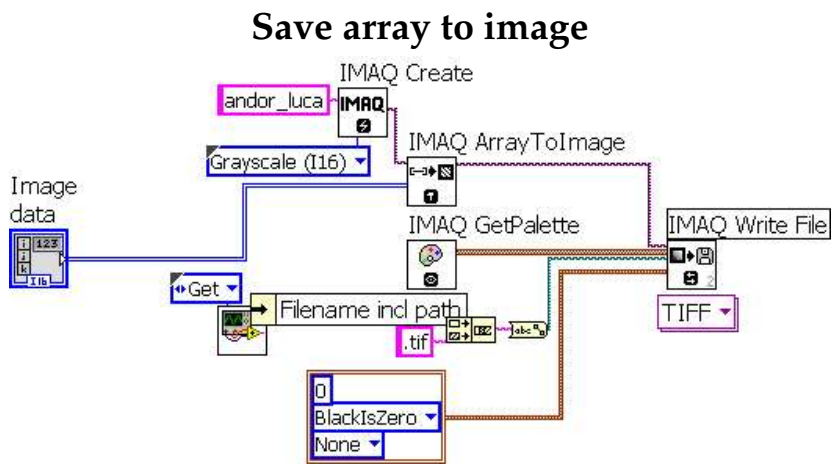

Figure 2.18: Save array to image

Save image acquisition time to textfile

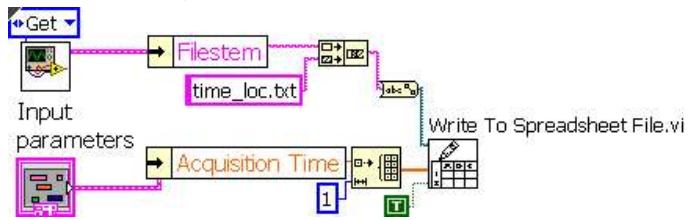

Figure 2.19: Save image acquisition time to textfile

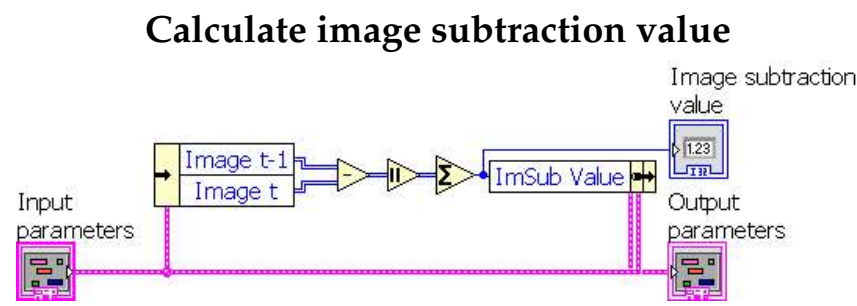

Figure 2.20: Calculate image subtraction value

Save image subtraction value

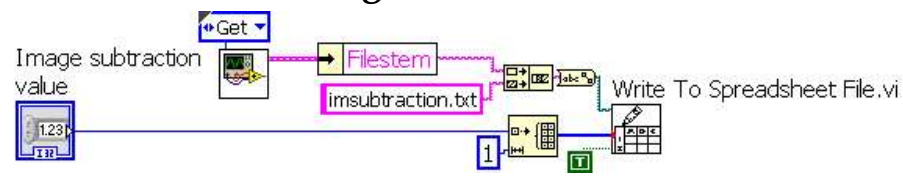

Figure 2.21: Save image subtraction value 
2.6. "C. elegans automated sleep deprivation"

\section{Select image subtraction value of SD animal

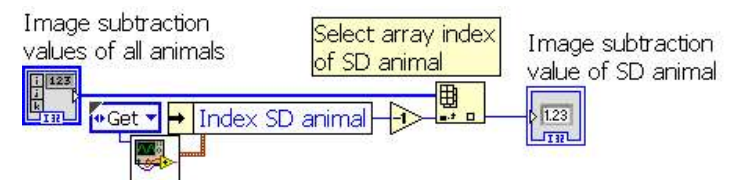

Figure 2.22: Select image subtraction value of SD animal 


\subsubsection{Sleep detection}

The "Sleep detection VI" (Figure 2.23) combines a verification of the sleep detection prerequisites, an automated and/or manual sleep detection, and a user defined optional delay of sleep detection. Local and global image subtraction arrays get updated ("Global and Local image subtraction arrays VI") and prerequisites to start sleep detection are evaluated ("Prerequisites to start sleep detection VI"). If the prerequisites are not fulfilled the VI terminates with the output "sleep detection"="false". If the prerequisites of sleep detection are fulfilled automated sleep detection criteria are evaluated ("Automated sleep detection mode VI"), manual sleep detection is evaluated ("Manual sleep detection VI") and an user defined optional delay for the sleep deprivation start is evaluated ("Delay sleep deprivation start VI"). The "Global and local image subtraction

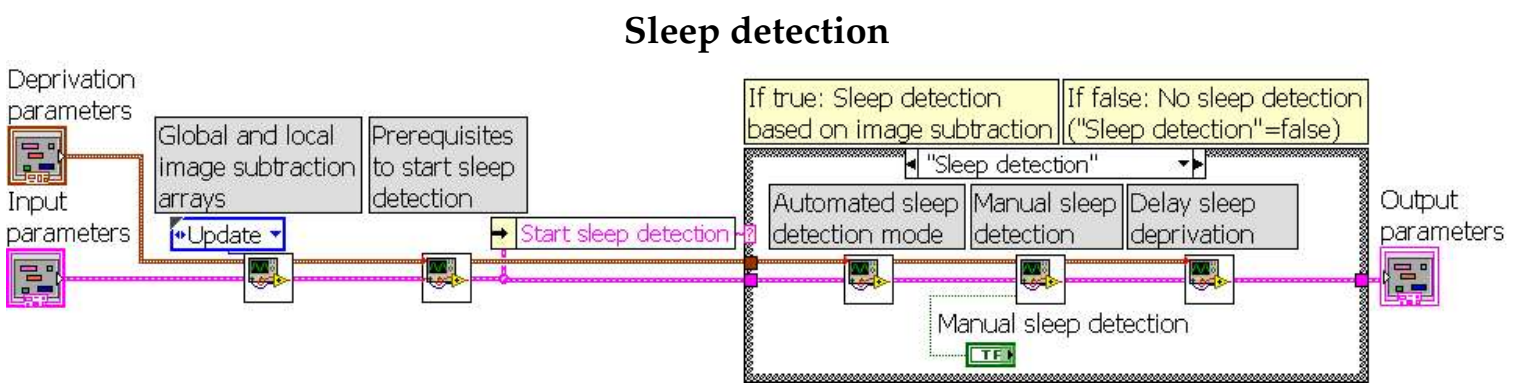

Figure 2.23: Sleep detection

array VI" (Figure 2.24) called with the update argument adds the latest image subtraction value of the SD animal to the global and local image subtraction arrays. During the update process the local image subtraction array is reduced by the oldest image subtraction value and the latest image subtraction value is added to it. The global image subtraction array gets extended by the latest image subtraction value. The "Prerequi-

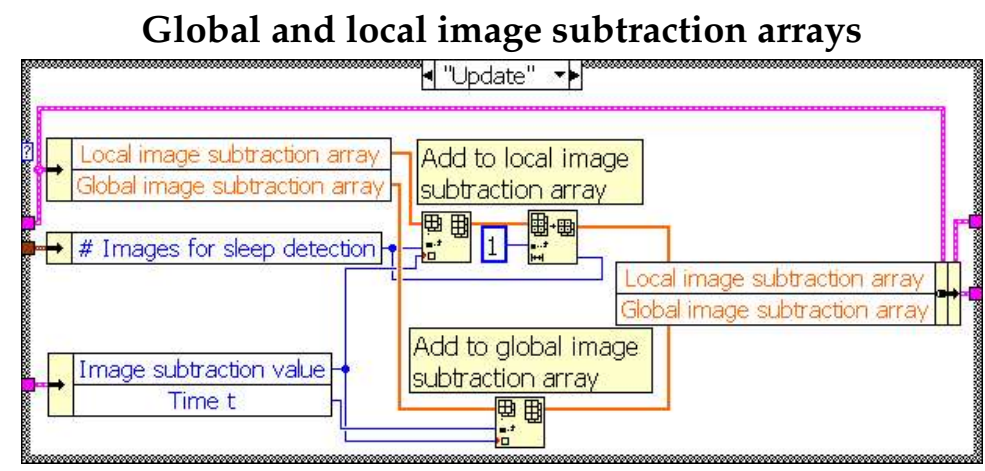

Figure 2.24: Global and local image subtraction arrays

sites to start sleep detection VI" (Figure 2.25) determines whether different prerequisite 
criteria are fulfilled to start sleep detection. It verifies that the start time for sleep detection is reached, that the minimum interval between TTL pulses is kept, and that neither the maximum number of adjacent TTL, nor the maximum deprivation time, nor the maximum number of TTL is reached. The "Automated sleep detection mode VI" (Fig-

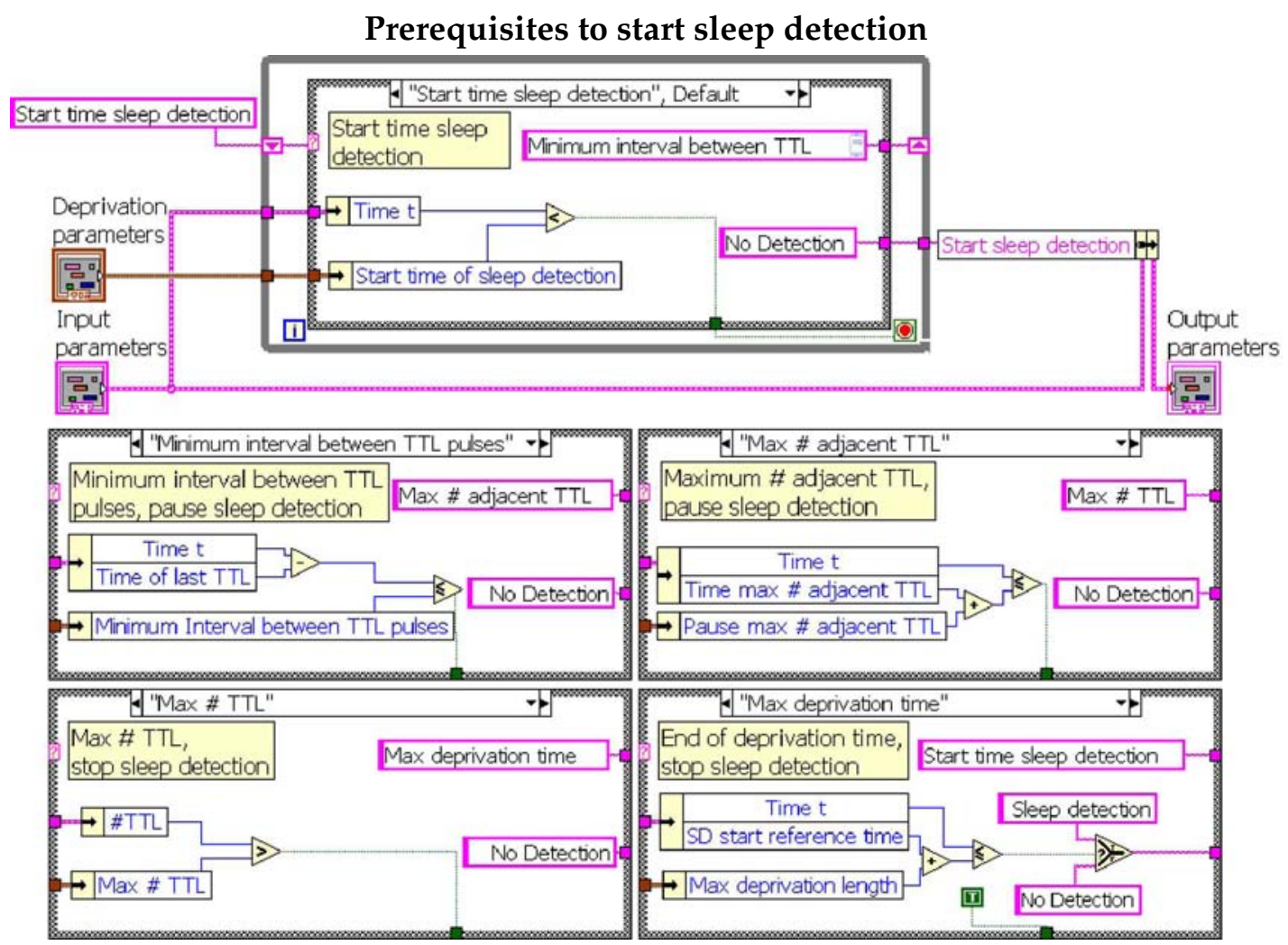

Figure 2.25: Prerequisites to start sleep detection

ure 2.26) determines which sleep detection criterion to employ. Total immobility ("Sleep detection criterion total immobility VI") is used as the sleep detection criterion for a user defined number of total immobility detections as reflected by the number of TTL signals ("\#TTL"). Once sleep has been detected and deprived more often a less restrictive sleep criterion is used ("Sleep detection criterion low mobility VI").

The "Sleep detection criterion total immobility VI" (Figure 2.27) evaluates the empirical sleep detection criterion based on image subtraction values, for details refer to section 3.1.3. The "Sleep detection criterion low mobility VI" (Figure 2.28) is detecting sleep if the mobility for only one timepoint is lower than a threshold.

The "Manual sleep detection VI" (Figure 2.29) determines, whether sleep has been detected manually by pressing the button "manual sleep detection" in the main front panel. The VI outputs "sleep detection"="true" if sleep has been either detected automatically and/or manually.

The "Delay sleep deprivation start VI" (Figure 2.30) verifies if a sleep detection gets 


\section{Automated sleep detection mode}

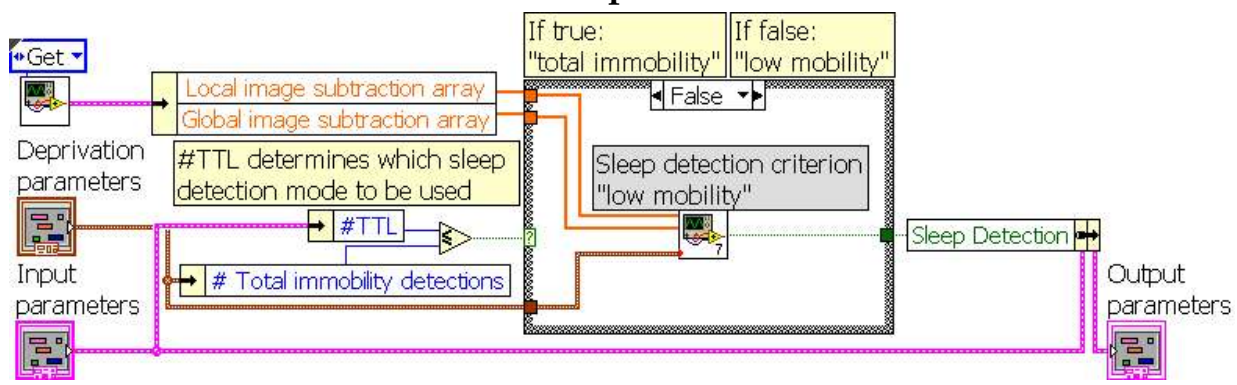

Figure 2.26: Automated sleep detection mode

\section{Sleep detection criterion total immobility}

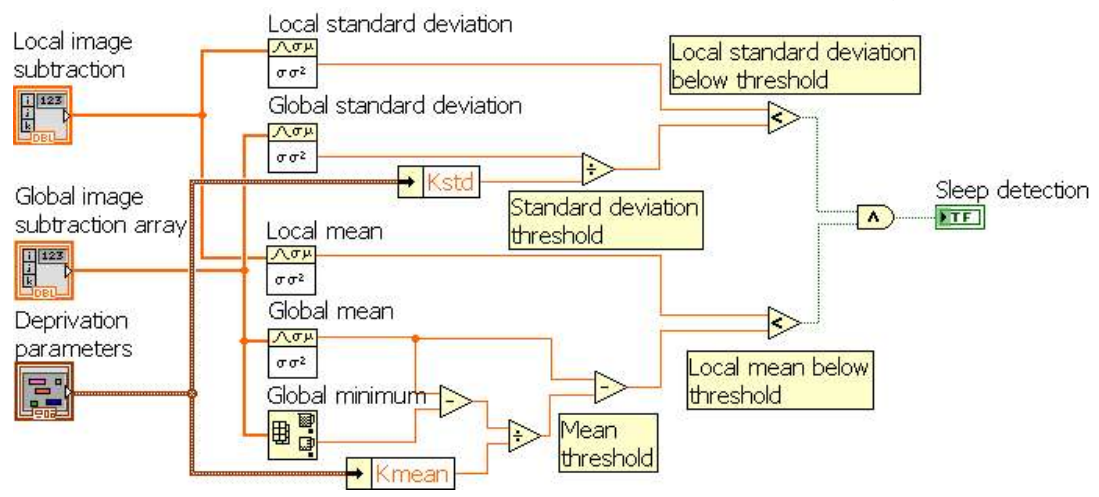

Figure 2.27

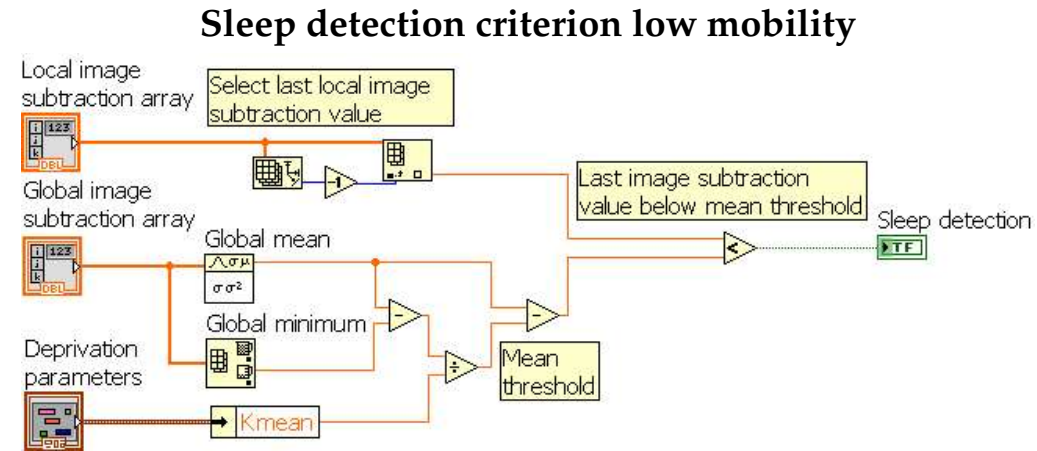

Figure 2.28: Sleep detection criterion low mobility

delayed by user defined optional parameters. First sleep detection parameters are updated ("Update sleep detection parameters VI"), then it is verified whether the start of sleep deprivation gets delayed by a certain amount of images ("Delay deprivation start by $x$ images VI") or of sleep detections ("Delay deprivation start by $x$ detections"). The 


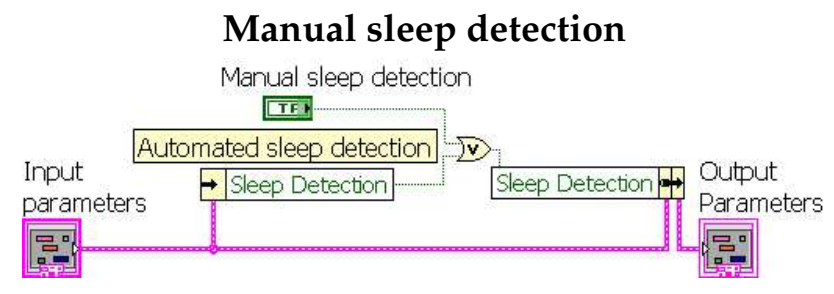

Figure 2.29: Manual sleep detection

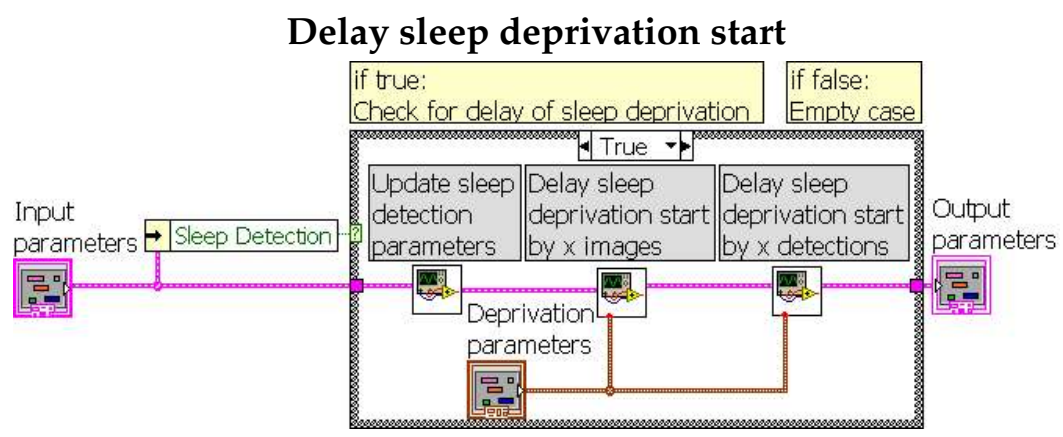

Figure 2.30: Delay sleep deprivation start

"Update sleep detection parameters VI" (Figure 2.31) increases the number of sleep detections by 1 and if this is the first sleep detection sets "Time of first sleep detection" to the current time.

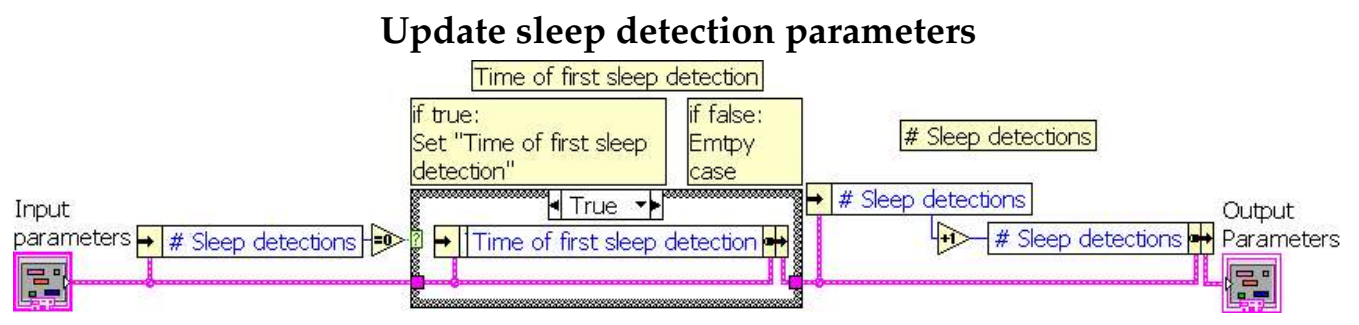

Figure 2.31: Update sleep detection parameters

The "Delay deprivation start by $x$ images VI" (Figure 2.32) verifies if the sleep deprivation start gets delayed by a user defined amount of time. The "Delay deprivation start by $x$ detections VI" (Figure 2.33) verifies if the sleep deprivation start gets delayed by a user defined amount of detections. 


\section{Delay deprivation start by $\mathrm{x}$ images}

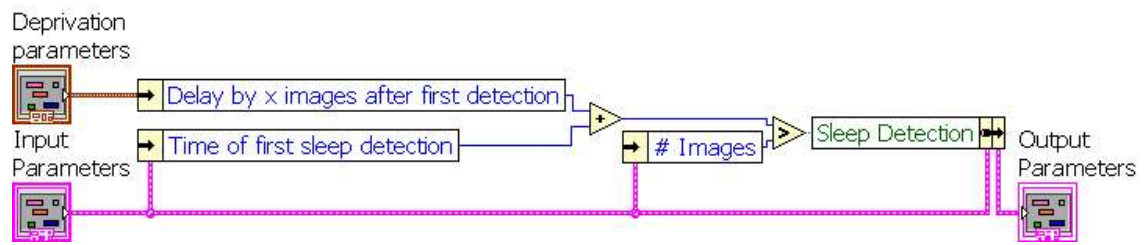

Figure 2.32: Delay deprivation start by $\mathrm{x}$ images

\section{Delay deprivation start by $\mathrm{x}$ detections}

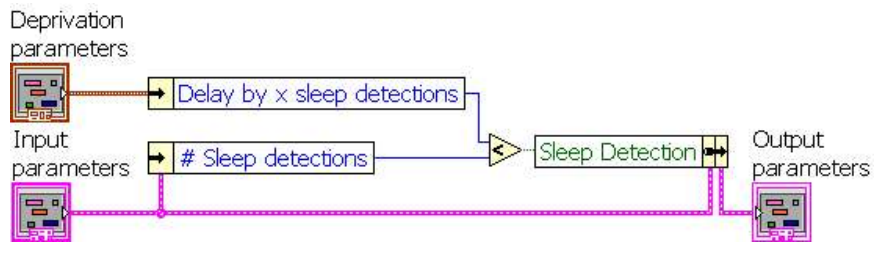

Figure 2.33: Delay deprivation start by $\mathrm{x}$ detections

\subsubsection{Control TTL protocol}

The "Control TTL protocol VI" (Figure 2.34) reads a list of TTL timings from a textfile, compares them to the current time $t$ and outputs "sleep detection"="true" if they coincide.

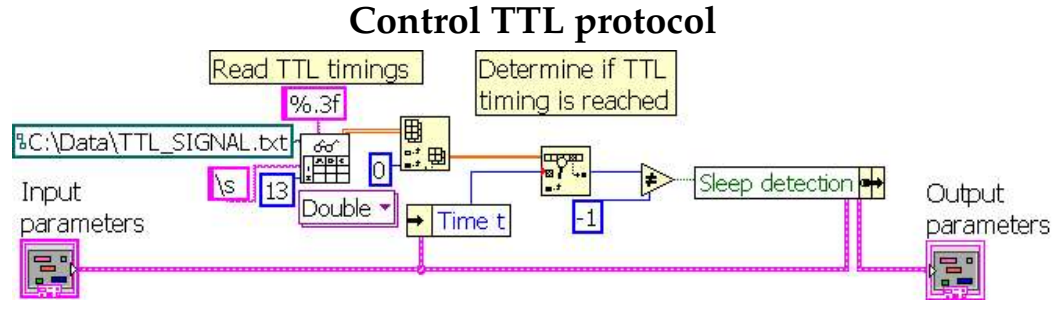

Figure 2.34: Control TTL protocol 


\subsubsection{Sleep deprivation}

The "Sleep deprivation VI" (Figure 2.35) saves and updates TTL parameters, triggers optional calcium-imaging, determines the deprivation method to be used and triggers the thereby specified TTL pulses.

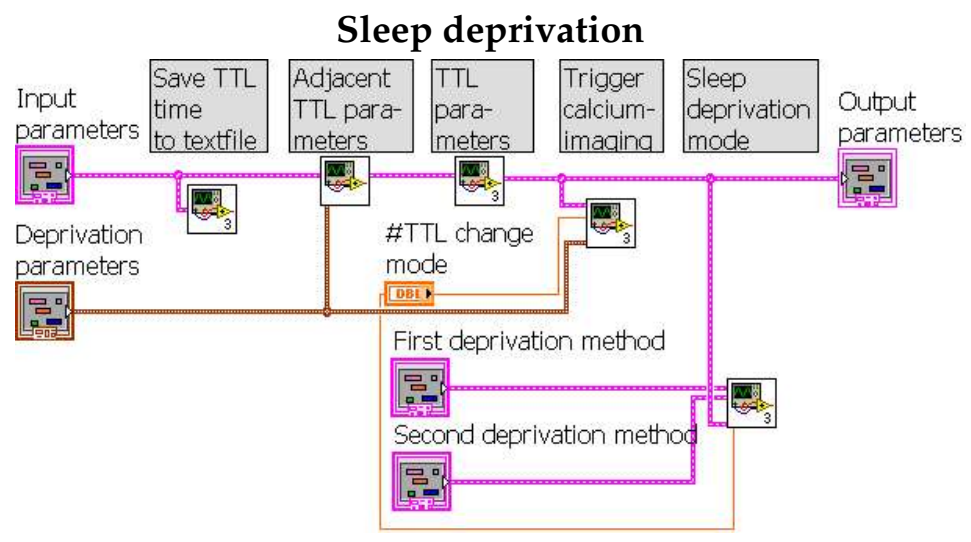

Figure 2.35: Sleep deprivation

The "Save TTL time to textfile VI" (Figure 2.36) saves the current time to the textfile "Strain-name-IndexSDAnimal-Date-TTL_SIGNAL.txt". The "Update adjacent TTL pa-

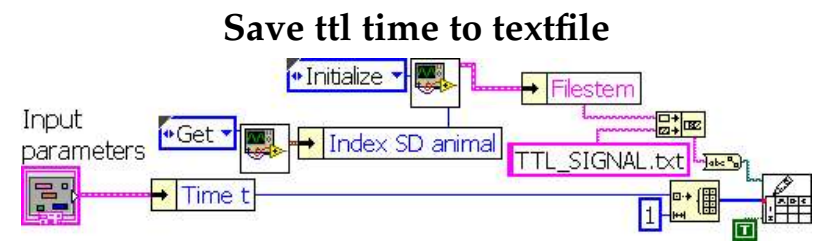

Figure 2.36: Save ttl time to textfile

rameters VI" (Figure 2.37) updates the number of adjacent TTL and if the number of adjacent TTL corresponds to the user defined maximum number of adjacent TTL it sets "time maximum \# adjacent TTL" to the current time.

The "Update TTL parameters VI" (Figure 2.38) updates the number of TTL, sets the time of the last TTL to the current time, and if the number of TTL corresponds to the user defined "\# TTL SD start refence time" it sets "SD start reference time" to the current time.

The "Trigger calcium-imaging VI" (Figure 2.39) triggers a user defined optional calciumimaging. Mode " 0 " corresponds to no calcium-imaging. Mode " 1 " outputs a single TTL pulse to the "external trigger" of a EMCCD-camera used for calicum-imaging. Mode "2" outputs a single TTL pulse to the "external trigger" of a EMCCD-camera used for calicum-imaging if the number of TTL is equal or bigger than "\# TTL change mode". 


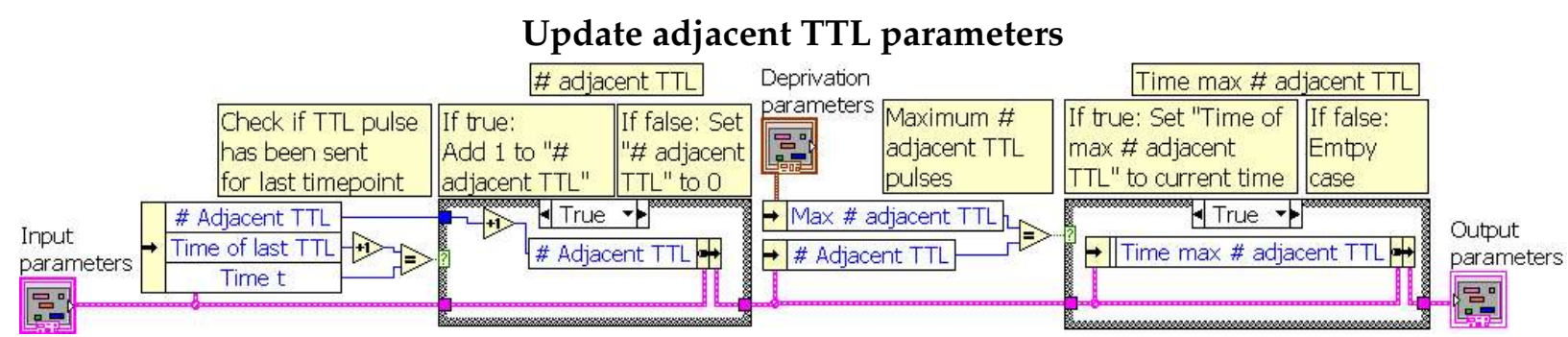

Figure 2.37: Update adjacent TTL parameters

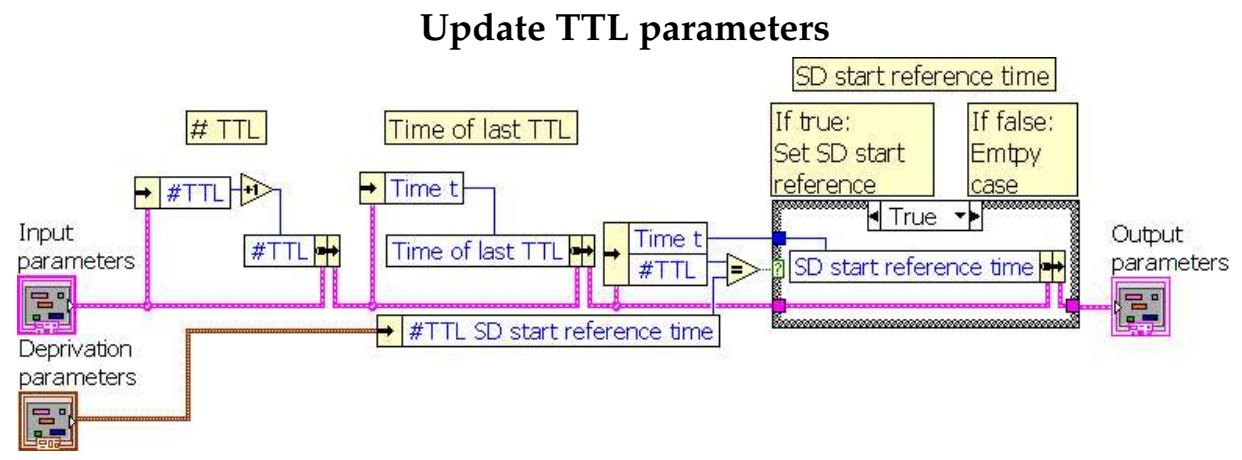

Figure 2.38: Update TTL parameters

\section{Trigger calcium-imaging}

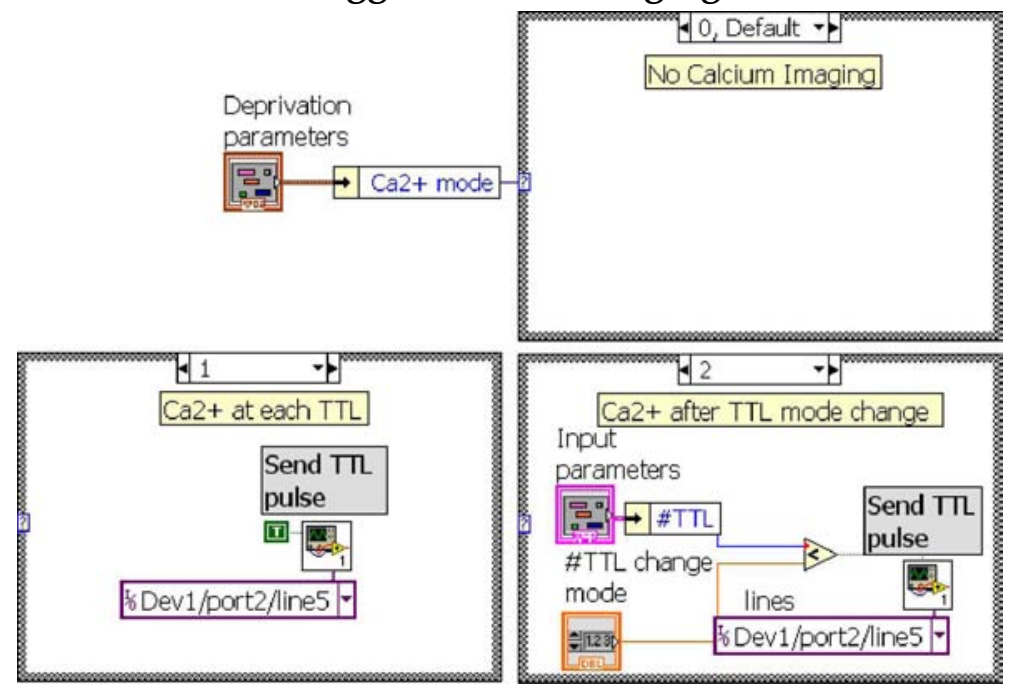

Figure 2.39: Trigger calcium-imaging

The "Sleep deprivation mode VI" (Figure 2.40) determines which one out of two user 
defined deprivation methods is used depending on the "\# TTL" compared to "\# TTL change mode". It then outputs a TTL pulse sequence with the parameters specified by the selected sleep deprivation method.

\section{Sleep deprivation mode}

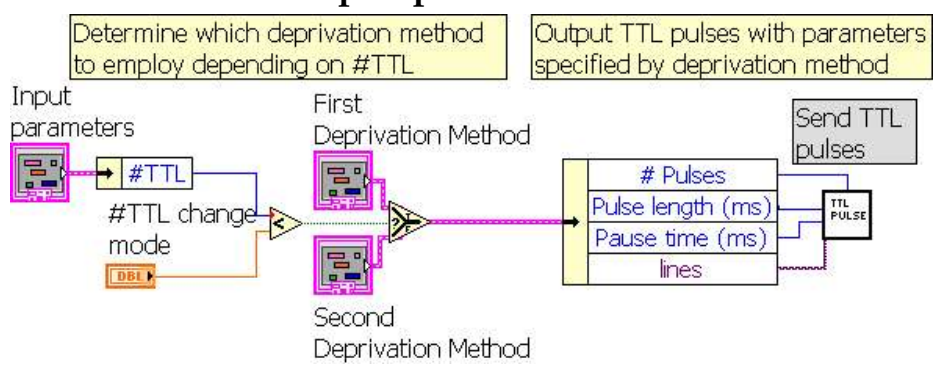

Figure 2.40: Sleep deprivation mode

The "Send TTL pulses VI" (Figure 2.41) outputs a sequence of TTL pulses defined by the user defined values of the deprivation method: "\# Pulses", "Pulse length (ms)", "Pause time (ms)", and "Line to output TTL".

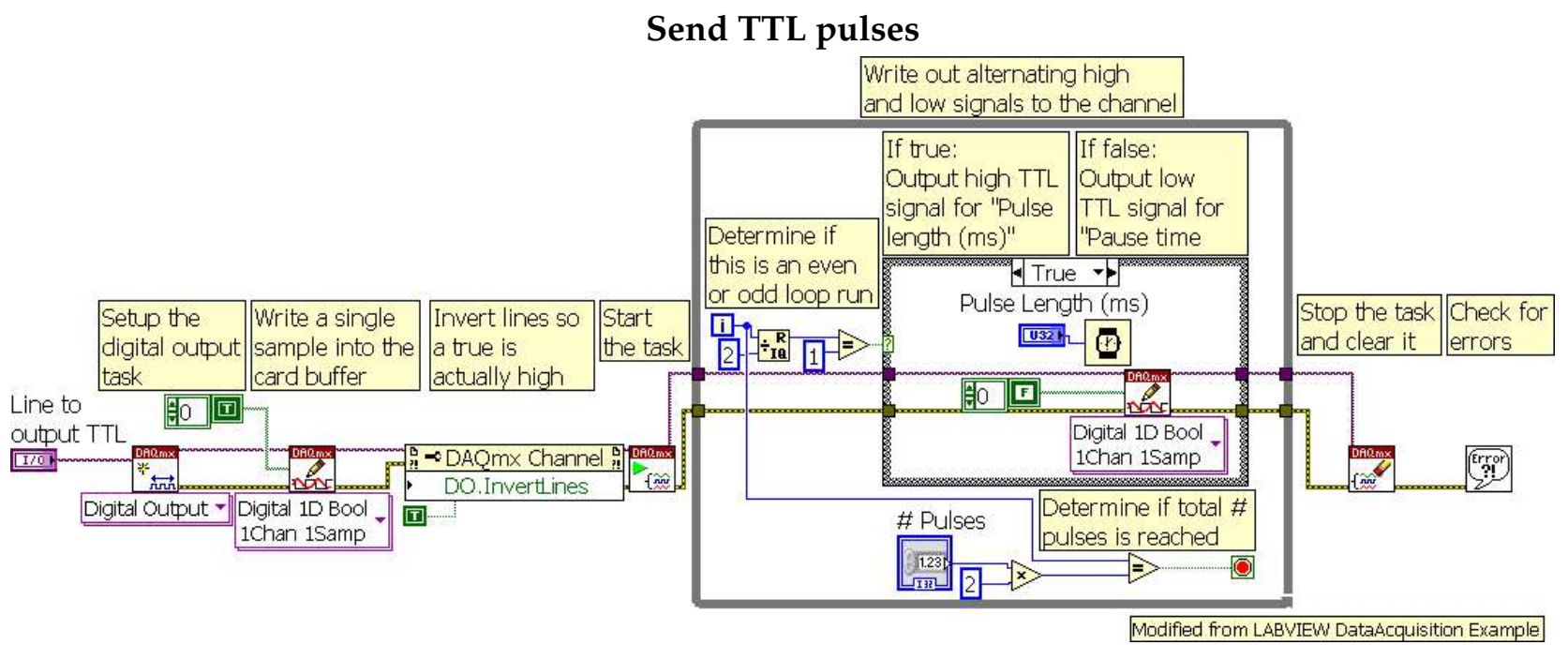

Figure 2.41: Send TTL pulses

\subsubsection{Update image \& exit condition}

The Update image \& exit condition VI replaces image $t-1$ by image $t$, increases the time $t$ by one, and verifies whether the user defined end measurement time (Max_num_runs) is reached (Figure 2.42). If the end measurement time is reached, the camera gets shut down using the ANDOR SDK VI ShutDown and the Main VI terminates. 
Update image \& exit condition

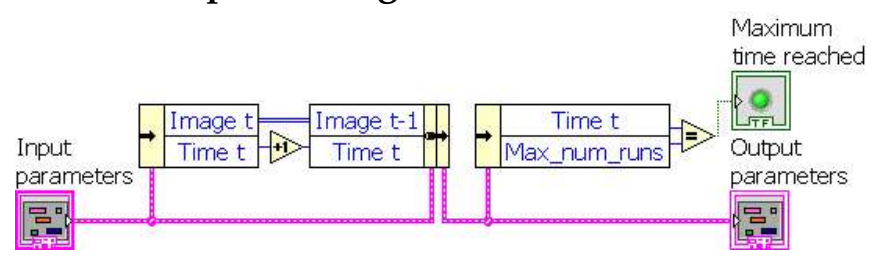

Figure 2.42

\subsubsection{Wait}

The "Wait VI" (Figure 2.43) delays the next image acquisition until the user defined frame rate is reached.

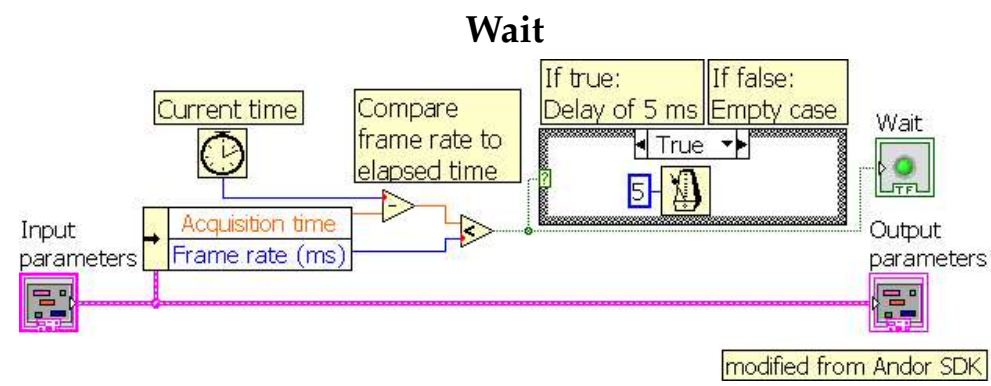

Figure 2.43: Wait

\subsection{9 "C. elegans automated sleep deprivation" interface}

"C. elegans automated sleep deprivation" program interface consist of a single control window 2.44. The controls can be divided in six major categories that are indicated in the figure by red capital letters:

- A: Display of camera image,

- B: Camera acquisition settings

- C: TTL-pulse settings

- D: General settings

- E: Sleep deprivation settings

- F: Start/End measurement 
2.6. "C. elegans automated sleep deprivation"

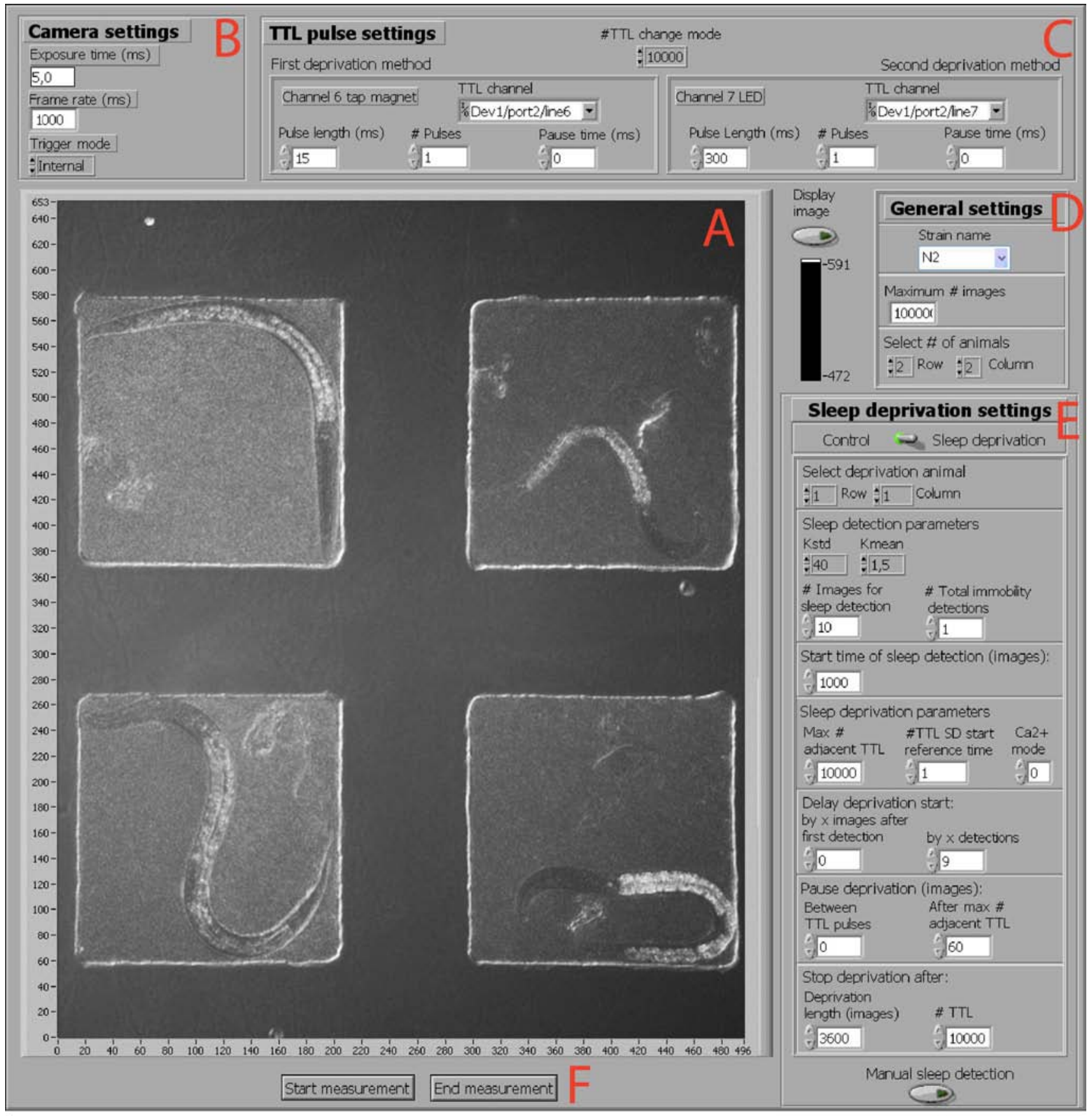

Figure 2.44: Control window - A: Display of camera image, B: Camera acquisition settings, C: TTL-pulse settings, D: General settings, E: Sleep deprivation settings, F: Start/End measurement 


\section{Display image}

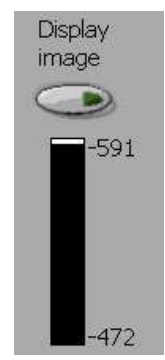

Figure 2.45: Display image

- Display image: Default (off), range (on, off).

If switched on newest image is displayed and gets updated by timing specified in "frame rate $(\mathrm{ms})$ ". Before starting the measurement display image should be switched off, to avoid delays in capturing images.

- Light intensity: range (20\%-80\% of saturation intensity).

Minimum and maximum pixel light intensity. If using an Andor Luca camera, intensities of about 7000-9000 are a good choice.

\section{Camera settings}

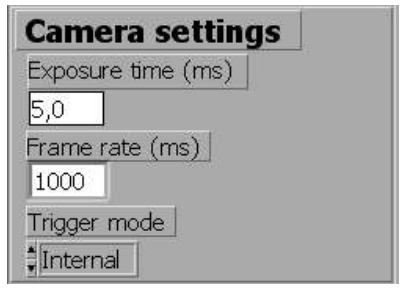

Figure 2.46: Camera settings

- Exposure Time (ms): Default value (5), range (1-10)

- Frame rate (ms): Default value (1000), range (500-4000)

- Trigger mode: Default Internal, range (Internal, External)

Trigger mode configures whether the image acquisition of the camera should be triggered by LABVIEW (Internal), or whether the camera gets trigger by a TTL signal (External).

\section{TTL-pulse settings}


2.6. "C. elegans automated sleep deprivation"

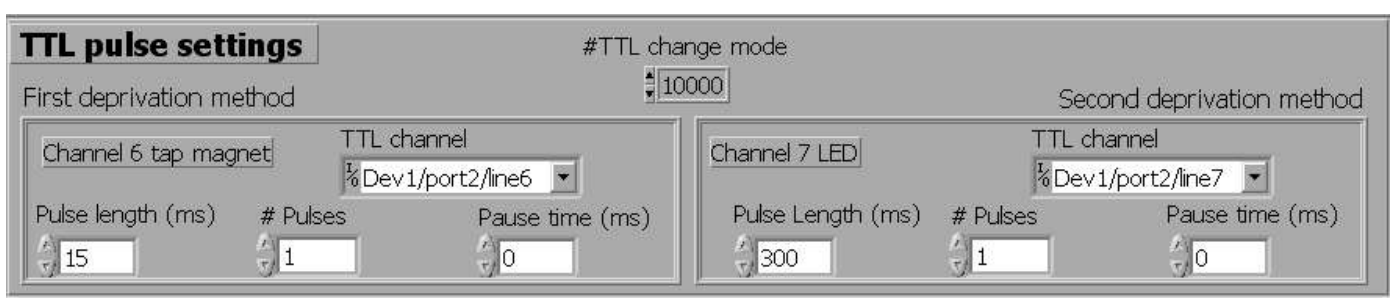

Figure 2.47: TTL pulse settings

- \# TTL change mode: Default value (10000), range (0-100000).

Number of TTL signals after which the deprivation method changes. Deprivation methods are defined by the following settings.

- First/second deprivation method:

TTL channel: Default value (Line6/Line7), range (Line1-Line8).

Channel of the data acquisition card that should output TTL signals. In our setup, currently line-6 is connected to a magnetic dish-tapper and line-7 to a LED.

Pulse length (ms): Default value (15/300), range (5-10000).

Duration of one TTL pulse in ms.

\# pulses: Default value (1/1), range (1-100). Number of separate TTL pulses that are output as one bout.

Pause time (ms): Default value (0/0), range(0-10000). Pause time between two TTL pulses that belong to one output signal.

\section{General settings}

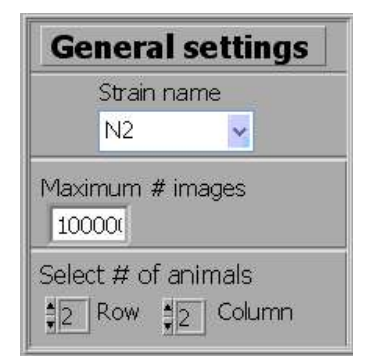

Figure 2.48: General settings

- Strain name: Default value (N2). range(any string)

The strain name is used together with the date as the name for the measurement. New C. elegans strain names can be added by right click, edit item.

- Maximum \# (number) images: Default value (100000), range(1000-200000).

The measurement will be stopped automatically if this value is reached. 
- Select \# of animals row/column: Default value (2/2), range(1-3, 1-3).

Sets the number of animals per row/column. Depending on the size of the camera chip and objective used, more than $3 \times 3$ animals can be imaged and sleep be detected.

\section{Sleep deprivation settings}

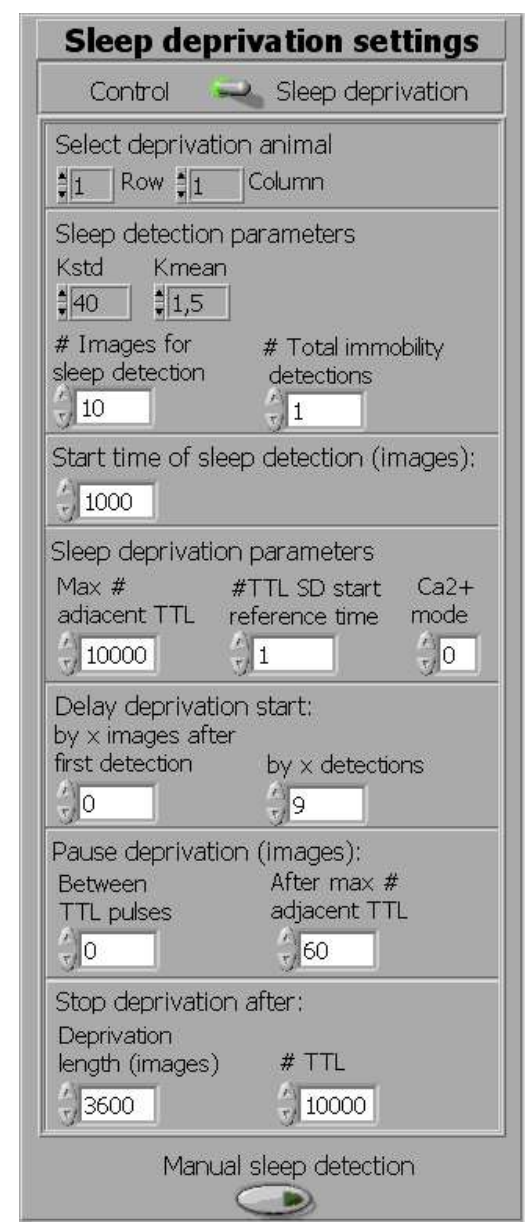

Figure 2.49: Sleep deprivation settings

- Mode: Default value (Sleep deprivation), range (Control, Sleep deprivation). The configuration "sleep deprivation" uses automated sleep detection and deprivation. The configuration "control" administers TTL signals according to a list of timepoints that it reads from a textfile.

- Select deprivation animal row/column: Default value (1/1), range (1/1-\# animals per row/column).

Sets animal in which sleep gets detected and sleep deprived. The index of row and column is starting with the animal on the down-left. In the displayed example image the animal on the top-left would be annexed with $(2,1)$. 
- Sleep detection parameters

$k_{s t d}$ : Default value (40), range (10-60).

Standard deviation constant for the total immobility sleep detection criterion. Lower values facilitate sleep recognition in light sleep mutants.

$k_{\text {mean }}$ : Default value (1.5), range (1-2).

Mean value constant for the total immobility sleep detection criterion. Higher values facilitate sleep recognition in light sleep mutants.

\# images for sleep deprivation: Default value (10), range (5-60).

Local time window for which the sleep detection criterion is evaluated. Smaller values facilitate sleep recognition in light sleep mutants, whereas bigger values assure in mutants with ectopic sleep phases or hypoactivity that sleep gets not falsely recognized.

\# (number) Total immobility detections: Default value (2), range (1-10000). Number of times the restrictive sleep detection criterion of total immobility is used. After this number of detections sleep recognition occurs if mobility is lower than a threshold once.

- Start time of sleep detection (images): Default value (1000), range (300-20000).

Time to start evaluation of the sleep detection criterion. Prevents too early false sleep detection. Ideally one sets this value about 2-3 hours prior to the estimated sleep start.

- Sleep deprivation parameters

Max \# (number) adjacent TTL: Default value (10000), range (1-10000).

Restricts the number of adjacent TTLs by forcing a pause of deprivation as specified below by the parameter "Pause deprivation after max \# adjacent TTL". The number of adjacent TTL being higher than 1 signifies that the animal did not react to the deprivation stimulus.

\# TTL start SD reference time: Default value (1), range (1-10).

Defines the timepoint of the $x$-th TTL as the beginning of sleep deprivation. This is used as the reference time for the deprivation length.

Ca2+ mode: Default value $(0)$,range $(0,1,2)$.

- Delay deprivation start

by $x$ images after first detection: Default value (0), range (0-7200).

Delays the sleep deprivation by at least this amount of time after the first sleep detection. Sleep detection possibly triggering sleep deprivation restarts after this delay has passed.

by $x$ detections: Default value (9), range (0-30).

Do not output a deprivation stimulus for the first $x$ detections. 
- Pause deprivation

between TTL pulses: Default value (0), range (0-600).

Forced minimum interval between two TTL pulses.

after max \# adjacent TTL: Default value (9),range (0-10).

Forced pause if "max \# adjacent TTL", as defined above in sleep deprivation parameters, is reached.

- Stop deprivation after

deprivation length: Default value (3600), range (300-10000).

Maximum duration of sleep deprivation. Sleep deprivation start is defined by the timepoint of the $x$-th TTL signal, see sleep deprivation parameter "\# TTL start SD reference time".

\# TTL: Default value (10000), range (10-10000).

Number of deprivation stimuli delivered after that deprivation gets stopped.

Manual sleep detection: For the timepoints this button is pressed sleep is detected. This can be used to as replacement of the automated sleep detection or in combination.

Example: Single worm sleep detection with default parameters

General settings

- Select \# of animals row/column: (1/1) Sleep deprivation settings

- Select deprivation animal row/column: (1/1)

Example: Multiple worm sleep detection with default parameters

4 animals, deprive the animal on the down-right.

General settings

- Select \# of animals row/column: (2/2)

Sleep deprivation settings

- Select deprivation animal row/column: $(1 / 2)$

Example: Externally triggered sleep detection with default parameters

Connect externally triggered BNC-cable to Trigger IN of camera

Camera Settings

- Trigger mode: External

Example: Control measurement using predefined stimulus protocol with default parameters

Sleep deprivation settings

- Mode: Control

Create textfile containing a list of timepoints (integer), seperated by line breaks. 


\subsubsection{Optimizing sleep deprivation}

Optimizing deprivation

Parameters for sleep deprivation

When to start sleep deprivation

- minimum start time for sleep deprivation

- first sleep detection $+x$ hours

- first sleep detection $+\mathrm{x}$ further sleep detections

Design of one stimulus

- pulse length, number of pulses, pause time between pulses

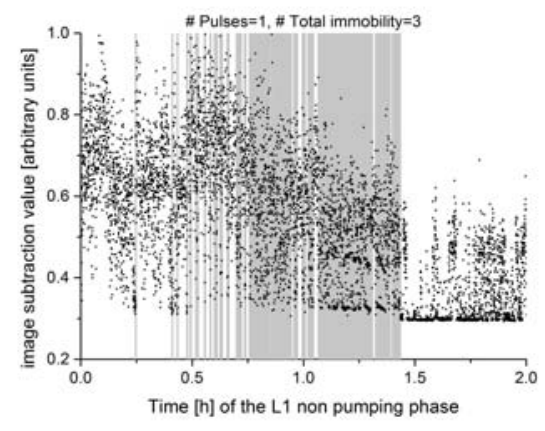

(a)

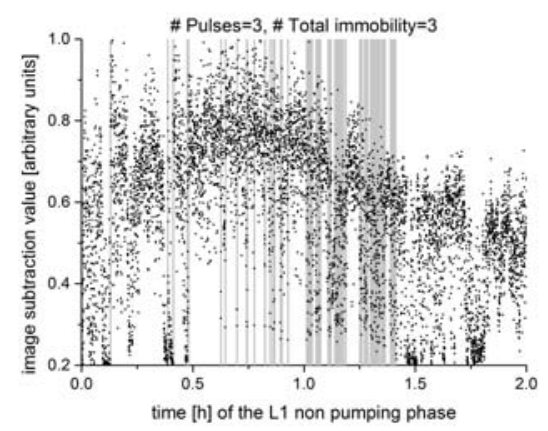

(b) 1

Figure 2.50: Design of the stimulus. A higher number (\#) of consecutive pulses at each sleep detection is more efficient than a single pulse. Shown is the image subtraction value time course for a single animal that receives each time sleep is detected either (a) a single mechanical stimulus or (b) three mechanical stimuli, with an interstimulus interval of one second. Mechanical stimulation is indicated by gray shading.

Lower the criterion for sleep detection once sleep has been detected for a certain number of times

- number of total immobility detections 


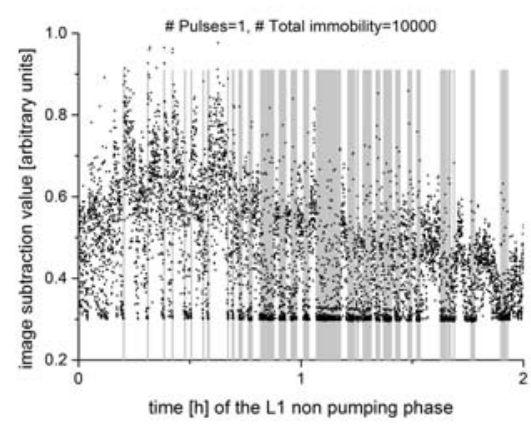

(a)

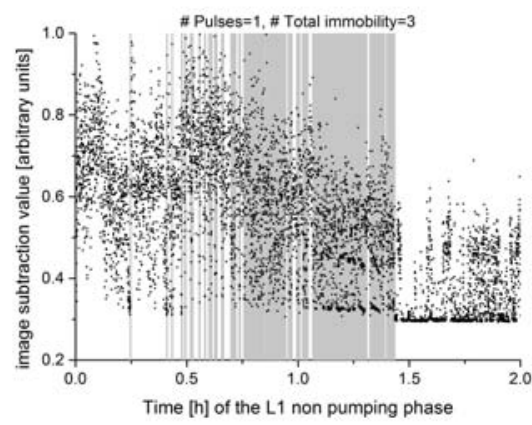

(b) 1

Figure 2.51: Lowering the sleep detection criterion after sleep has been detected several times yields better sleep deprivation results. Shown is the image subtraction value time course for single animals using either the sleep detection criterion of (a) total immobility only or (b) total immobility for the first three sleep detections followed by low mobility as the sleep detection criterion. Mechanical stimulation is indicated by gray shading.

Limitation of stimulation during sleep deprivation

- forced pause of $\mathrm{x}$ seconds between two stimulations

- forced pause of $x$ seconds if a certain number of sequential stimulations is reached

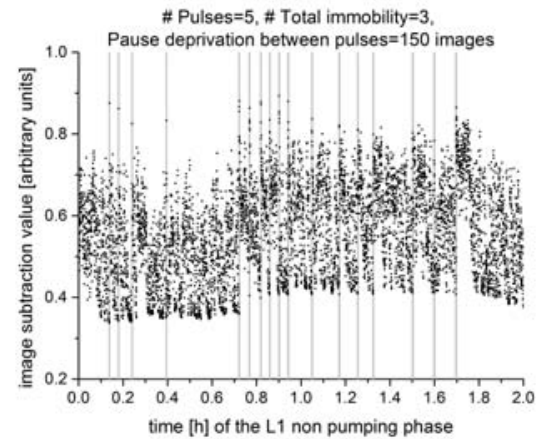

(a)

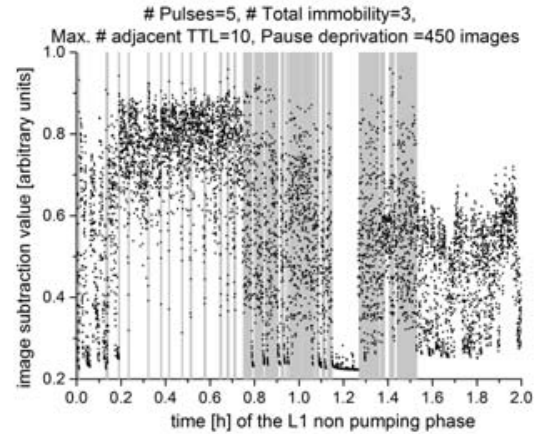

(b) 1

Figure 2.52: Limitation of stimulation during sleep deprivation. Shown is the image subtraction value time course for single animals pausing deprivation (a) after each stimulation and (b) if a maximum number of ten adjacent stimulations is reached. Mechanical stimulation is indicated by gray shading. 
When to stop sleep deprivation?

- after a certain time of deprivation is reached.

- after a certain number of taps has been delivered.

- if the animal did not react to a certain number of taps.

\subsection{Analysis of behavioral and neuronal activity}

For nose speed measurements, the nose was tracked manually, using the FIJI plugin "manualtracking" (http://rsb.info.nih.gov/ij/plugins/track/track. html).

Image subtraction was done using LABVIEW or MATLAB.

RIS position was detected using a custom written MATLAB algorithm, that can be found in appendix D. Briefly, detection of RIS was based on its position relative to the cluster of AIB and RIB neurons. RIA detection was based on a custom written pattern recognition algorithm. I manually verified the correct identification of the RIS and RIA signal. For images in which automatic detection of RIS was not possible, the position was manually tracked. ALM, PLM, and PDA neuron positions were tracked manually using a MATLAB algorithm. To obtain the neuronal activity for a given time point, the neurons were cut out using a MATLAB routine, the high intensity pixels corresponding to the neuron were averaged and the average value of low intensity pixels of the background was subtracted.

\subsection{Statistical analysis}

Standard statistical tests were performed using ORIGIN. Significance levels of 0.05, 0.01 and 0.001 were used and significant results are indicated with asterisks. Error bars indicate the standard error of the mean (SEM). Boxplots have lines at the lower quartile, median and upper quartile values and whiskers indicating variability outside the upper and lower quartiles. For all paired datasets the paired Wilcoxon rank test was used to assess significance levels. For unpaired datasets the two sample t-test with Welch correction was used. Correlation was assessed using the two tailed Spearman test.

\subsection{Transcriptional profiling using RNA Sequencing}

I collected wild-type and lim-6(nr2073) C. elegans threefold stage eggs manually using a pick. For both strains three replicates of 2000 eggs were collected. RNA was purified 
using the TRIzol protocol (Invitrogen).

The Transcriptome Analysis Laboratory Göttingen performed the cDNA library preparation, and RNA sequencing as previously described [78]. Statistical analysis was done by Klaus Jung (Medical Biometry and Statistical Bioinformatics Facility University of Goettingen). Resulting sequence reads were trimmed according to phred scores using the software flexbar and aligned to the reference genome (WormBase WS170) by the software STAR [79], [80]. The mapped reads were counted by htseq (http: //www-huber.embl. de/users/anders/HTSeq/doc/overview.html). Statistical analysis was done using the software $\mathrm{R}$ (version 2.15, www.r-project.org and Rpackages from www.bioconductor.org. Negative binomial models were used to detect differentially expressed genes [81]). P-values were adjusted by the method of Benjamini and Hochberg, for controlling the false discovery rate at the 5\% level [82].

RNA Sequencing of the light-sleep mutant aptf-1(gk794) has been done simultaneously with my RNA Sequencing by Michal Turek. 


\section{Chapter 3}

\section{Results}

\subsection{Automated sleep recognition in C. elegans}

\section{C. elegans sleep specific behaviors and their suitability for sleep recognition}

Automated sleep recognition requires a robust detection criterion and should not disturb the animal. Possible criteria for the detection of sleep are the non pumping pharynx and the reduced mobility as described by Cassada and Russel in 1975 [37]. A sleep specific posture and an increased arousal threshold are further readouts for the

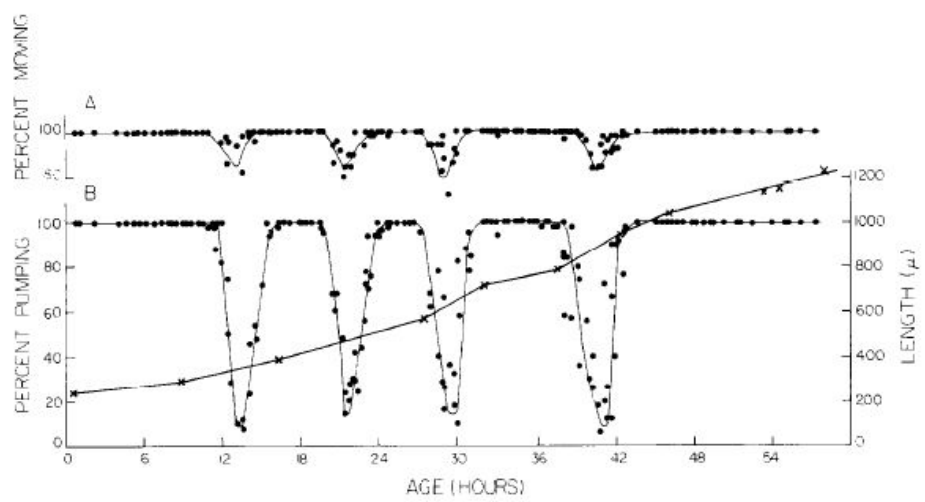

Figure 3.1: C. elegans has a reduced mobility and pumping frequency during sleep. Reproduced from Cassada and Russel [37].

sleep phase. Possible sleep recognition criteria are summarized in the following table 3.1. In the following I will discuss which of this sleep specific behaviors is best suited to
Reduced mobility
Non pumping pharynx
Sleep specific posture
Increased arousal threshold

Table 3.1: Possible sleep recognition criteria

underlay automated sleep detection. The increased arousal threshold can be excluded 
as a criterion for sleep detection, because determining the arousal threshold would mean to disturb the animal. Detection of the pharynx is difficult because of its small size. Therefore I will not pursue this idea. Identification of a sleep specific posture requires the detection of the complete outline of the worm. For our experimental setup this is a challenging task. On the other hand quantifying the mobility of the animal seems to be feasible.

\subsubsection{Automated identification of the worm outline}

Quantification of the posture and exact mobilities of the worm requires the detection of the worm outline. There are a multitude of wormtrackers readily available, for an overview please see review by Husson [83]. However these trackers have been optimized for a freely moving animal on a plate observed with low resolution and not for our agarose microcompartment setup. Attempts to use several worm trackers, including the wormtracker $2.0 \mathrm{http}: / / \mathrm{www} \cdot \mathrm{mrc}-\mathrm{lmb} . \mathrm{cam} . \mathrm{ac} \cdot \mathrm{uk} /$ wormtracker have failed to detect the worm in our experimental setup. This is mainly to the bad contrast of the worm that is surrounded by E. coli bacteria. One possibility to increase the contrast between bacteria and the nematode is to generate transgenic animals that express a fluorescent protein in vast parts of the animal.

\section{Worm detection by expressing a fluorescent protein in muscle tissue.}

We reported that the nematode has a sleep specific posture [42]. To show this, I extracted the posture of the worm from images expressing GCaMP3.35 in body wall muscles (Figure 3.2a). Briefly, I extracted the skeleton of the worm using a "Canny-Edge" algorithm implementation in MATLAB (Figure 3.2b). Then I dilated the obtained outline followed by a thinning operation to obtain the skeleton (Figure 3.2c, 3.2d). Manual verification of the resulting skeletons showed that for $26 \%$ of the images the algorithm did not produce a correct result. This mainly occurred when the worm's nose was touching its tail.

Using a fluorescence protein facilitates the detection of the worm. However the automated extraction of the worm posture was not robust enough to be used for sleep detection. Moreover the blue/green light required to excite the fluorophore possibly disturbs the animal and complicates simultaneous fluorescence imaging of neuronal activity. Therefore I did not pursue this idea. 
(a)

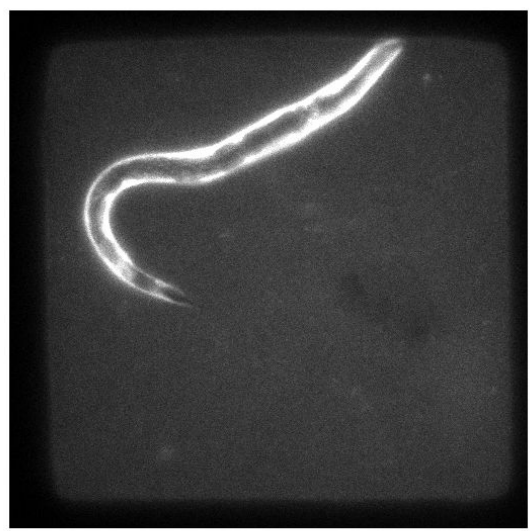

(c)

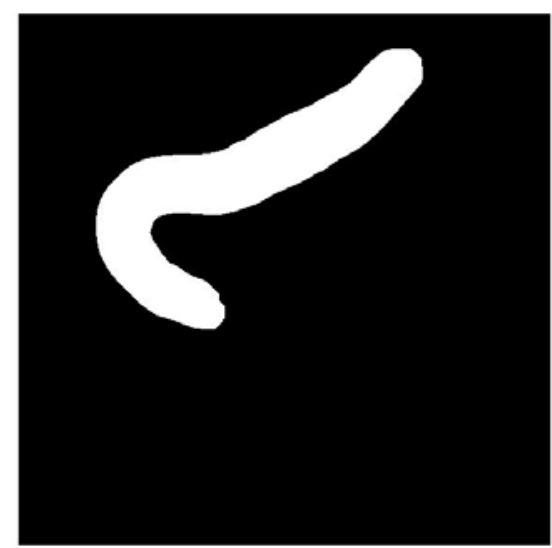

(b)

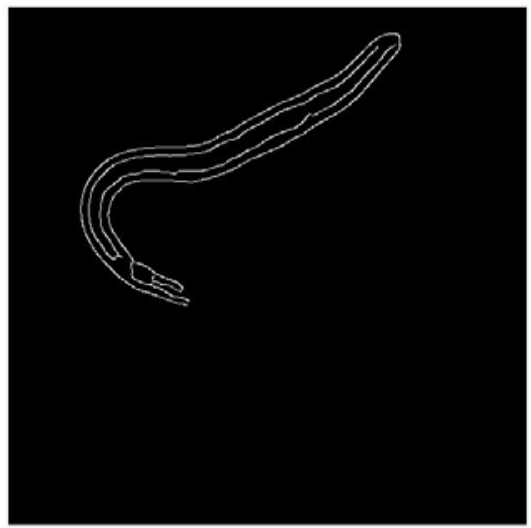

(d)

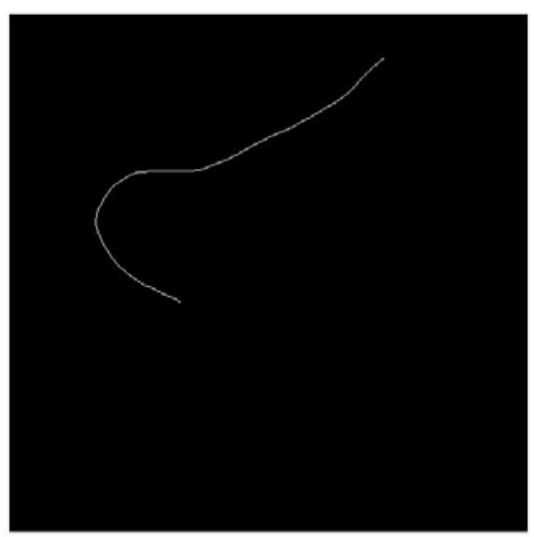

Figure 3.2: Extraction of the worm using a fluorescent protein. (a) Fluorescence image of a worm expressing GCaMP3.35 in body wall muscles. (b) Extraction of the midline of the worm using the "Canny-Edge" algorithm. (c) Dilation operation of the outline resulting in a blowup version of the worm's shape. (d) Thinning operation leading to the skeleton of the worm.

\section{Worm detection using high DIC contrast}

As existing $C$. elegans trackers were not able to extract the outline of the worm in our microcompartment setup, I decided to write my own worm detection alogrithm adapted to our experimental setup. The first thing to decide on was what kind of light microscopy imaging method to use. I tested if brightfield or differential interference contrast (DIC) microscopy is suited (Figure 3.3a, 3.3b). However, despite considerable 
effort I did not manage to extract the worm outline from these pictures robustly. By changing the alignment of the two polarizing filters used for DIC imaging I obtained a high contrast DIC image (Figure 3.3c).

(a)

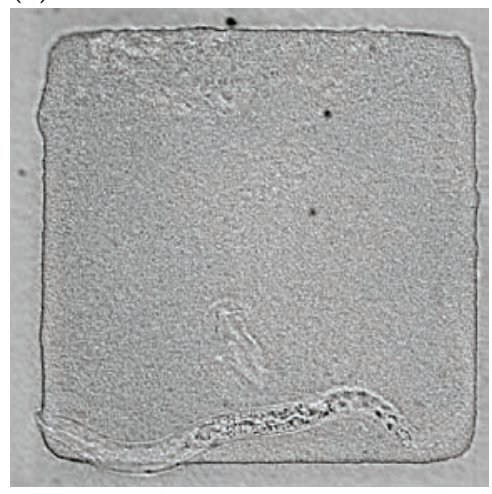

(b)

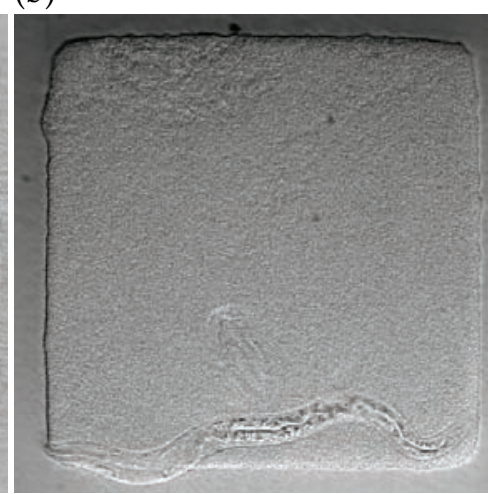

(c)

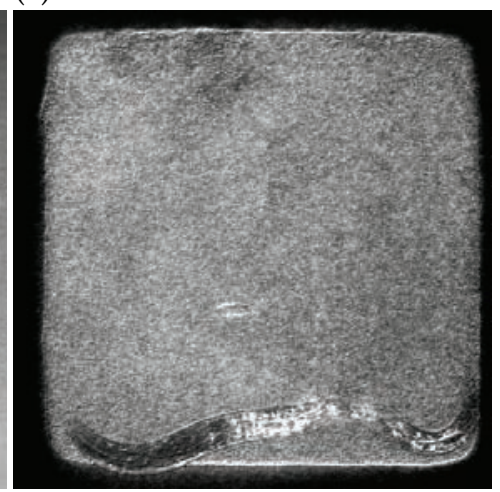

Figure 3.3: Brightfield and DIC images using different phase difference of a worm surrounded by E. coli OP 50 bacteria in a agarose microcompartment. (a) Brightfield image, (b) standard contrast DIC image, and (c) high contrast DIC image.

In this configuration the worm's nose is appearing darker than the background E. coli bacteria. I wrote a first worm nose detection algorithm in MATLAB, that could correctly identify the worm's nose in about $90 \%$ of the cases correctly. To improve this code to allow a more robust detection, I contacted the two computational scientists Boris Busche and Jan-Martin Kirves. Their work resulted in the "wormTracker", an automated nose detection program based on the $C$ programming language. It extracts the skeleton (midline) of the worms nose from the original high contrast DIC image (Figure 3.4). An improved version of the "wormTracker" is developed by Birk Urmersbach and is short before finalization. Briefly, it normalizes and inverts the original image (Figure $3.4 \mathrm{~b}$ ). Followed by a thresholding and thinning and dilation operation (Figure $3.4 \mathrm{c}$ and 3.4d). Then it selects the largest cluster and performs a skeletonization operation (Figure 3.4e). Figure 3.4f shows the overlay of the detected skeleton with the orignal DIC image. The "wormTracker" allowed an improved detection of the nose but still was not robust enough to be used for automated detection. False results were produced when the tail was touching the nose. 
(a)

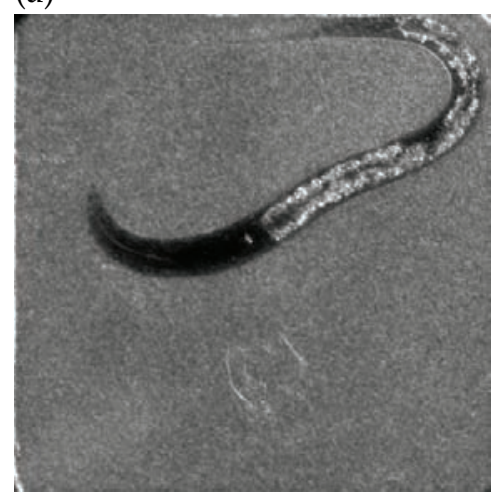

(d)

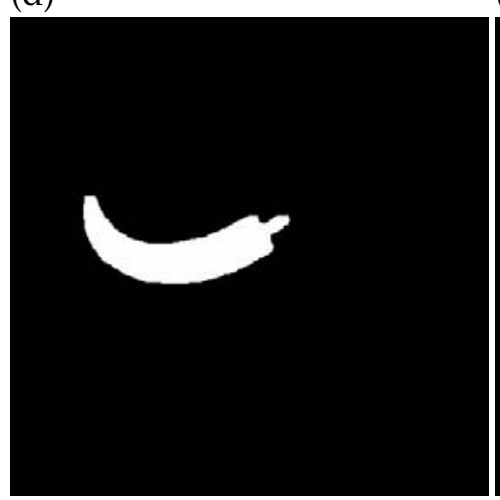

(b)

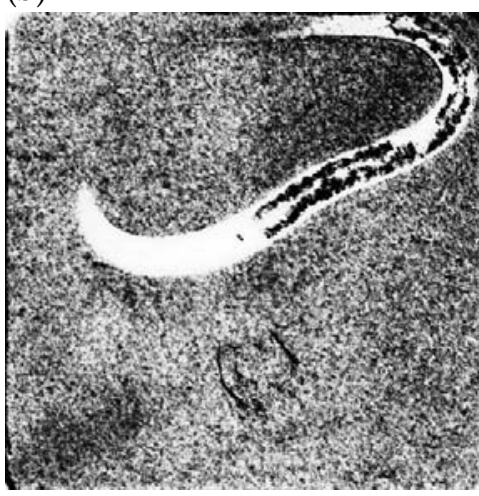

(e)

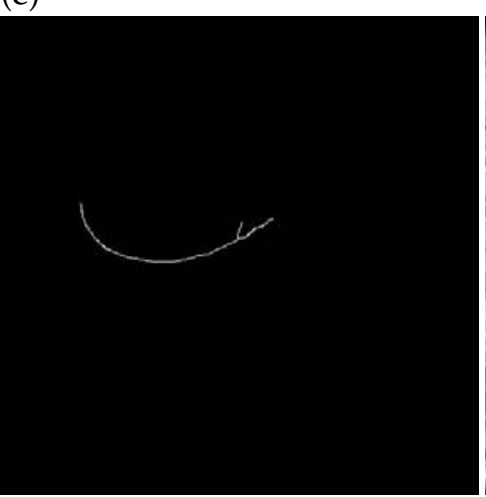

(c)

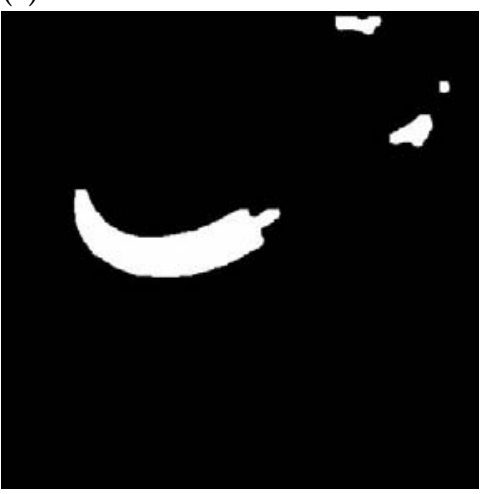

(f)

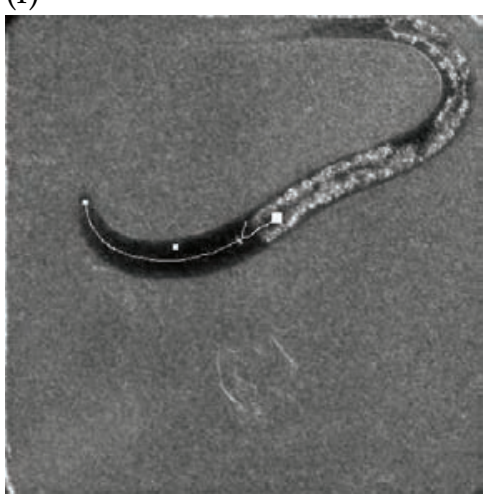

Figure 3.4: Extraction of the worm's nose based on high contrast DIC image. (a) DIC image with high contrast. (b) Normalization and inversion operation. (c) Thresholding, thinning and dilating operation. (d) Selection of the largest cluster. (e) Skeletonization operation. (f) Overlay of detected skeleton and DIC image.

\subsubsection{Image subtraction as a readout for mobility}

As the fully automated extraction of the worm outline for our experimental setup appeared to be a challenging, time consuming task I investigated whether there are simpler ways to robustly characterize the mobility of the worm. The variation between two consecutive images is reflecting the change in position of the worm, thus its mobility. I therefore investigated whether image subtraction, the pixel wise evaluation of the variation of two consecutive images can be used to robustly detect immobility.

The image subtraction value at the time point $t$ for the pixel $(i, j)$ is equal to the absolute difference of the pixel $(i, j)$ value for the time points $t$ and $t+1$.

$\operatorname{ImSub}(t)_{i j}=\left\|\operatorname{Im}(t+1)_{i j}-\operatorname{Im}(t)_{i j}\right\|$, with $\mathrm{t}$ : Point in time, $\mathrm{i}, \mathrm{j}$ : Indices of pixel values 
By summing up the image subtraction values of all pixels one obtains the image subtraction value of the entire picture.

$$
\operatorname{Imsub}(t)=\sum i j \operatorname{ImSub}(t)_{i, j}
$$

In figure 3.5 a visual representation of image subtraction is shown.
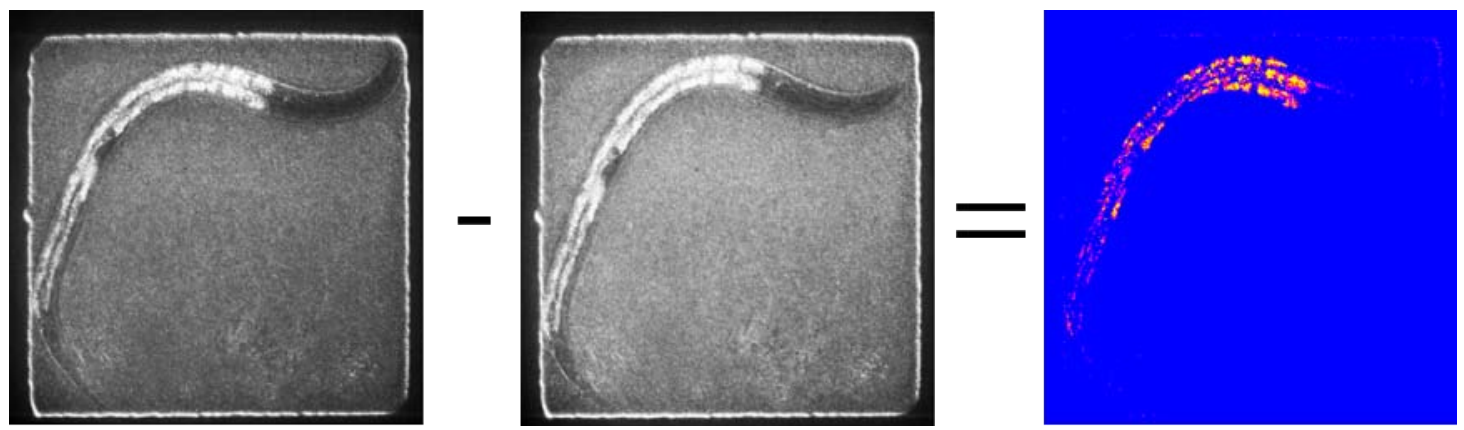

Figure 3.5: Visualization of image subtraction. Two consecutive frames captured with a $\Delta T$ of $1 \mathrm{~s}$ and their corresponding image subtraction picture during L1 wake. Blue corresponds to low, red to high differences between pixel values.

To quantify how well image subtraction is reflecting mobility I compared manually tracked nose velocities to their corresponding image subtraction values. Image subtraction is closely reflecting nose velocity. The wake-sleep-wake time course of image subtraction and nose velocity show a very similar pattern (Figure 3.6a). Both show a dip that corresponds closely to the sleep period. The graphical representation of image subtraction as a function of nose velocity indicates as well that they correlate (Figure 3.6b). The Spearman rank test gives indeed a positive correlation between image subtraction and nose velocity. The corresponding Spearman correlation coefficient is 0.595. 
(a)

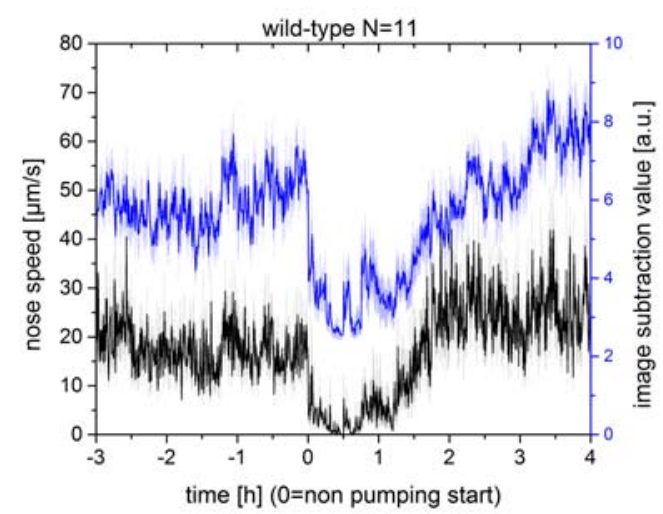

(b)

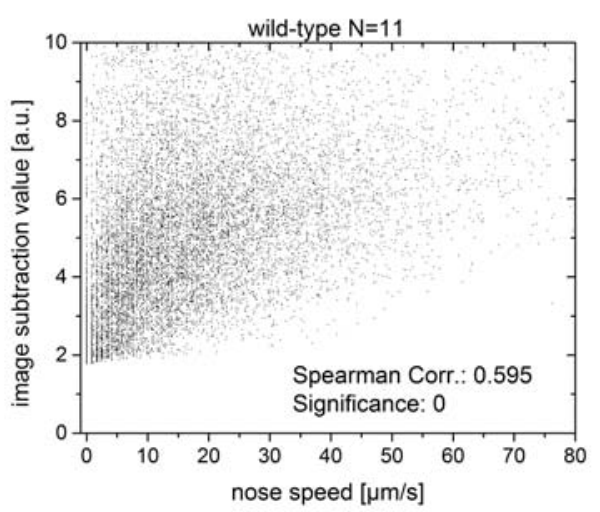

Figure 3.6: Nose speed and image subtraction are correlated. Shown is the (a) time course and (b) a histogram of nose speed and image subtraction values for $\mathrm{N}=11$ animals. Correlation was assessed using a two tailed Spearman test.

\subsubsection{A sleep criterion based on image subtraction}

After having shown that image subtraction is a measure for the mobility of the worm, the next question was if image subtraction permits to robustly recognize the sleep state of the worm. Recognition of the sleep state will be based on the low mobility of the worm and the resulting low image subtraction values. The questions to answer are therefore: First, Is low immobility a robust indicator for the sleep state. Second, Is the method of image subtraction suited to detect this immobility during sleep. Figure 3.7 is a visualization of how image subtraction ideally identifies the sleep like state.

The sleep detection criterion should be applicable to a wide range of sleep mutants, varying illumination settings and magnifications. Therefore I decided not to use an absolute threshold, but relative thresholding dependent on prior mobility. I established the sleep criterion empirically based on a dataset of the wake sleep cycle of ten animals. I annotated sleep and wake timings and tested which tresholding criteria best detected the sleep onset and did not result in false positives during wake state. I finally decided to use two separate thresholds. For a local time window to be defined, the standard deviation has to be smaller than a fraction of the global standard deviation and the mean value has to be smaller than a fraction of the global mean value minus the difference between the global mean and the global minimum (Equations 3.1).

$$
\begin{aligned}
\operatorname{std}(\text { local }) & \leq \operatorname{std}(\text { global }) / k_{\text {std }} \\
\operatorname{mean}(\text { local }) & \leq \operatorname{mean}(\text { global })-(\text { mean }(\text { global })-\min (\text { global })) / k_{\text {mean }}
\end{aligned}
$$

The second equation can be rewritten as follows

$$
\operatorname{mean}(\text { local }) \leq\left(\left(k_{\text {mean }}-1\right) * \text { mean }(\text { global })+\min (\text { global })\right) / k_{\text {mean }}
$$




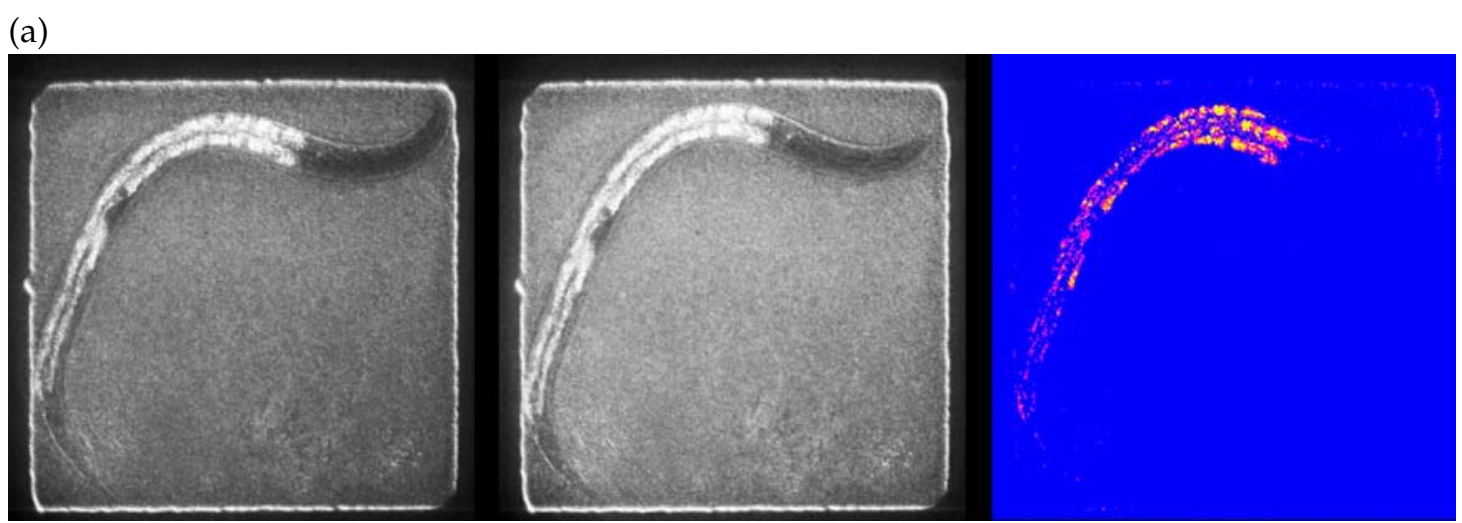

(b)

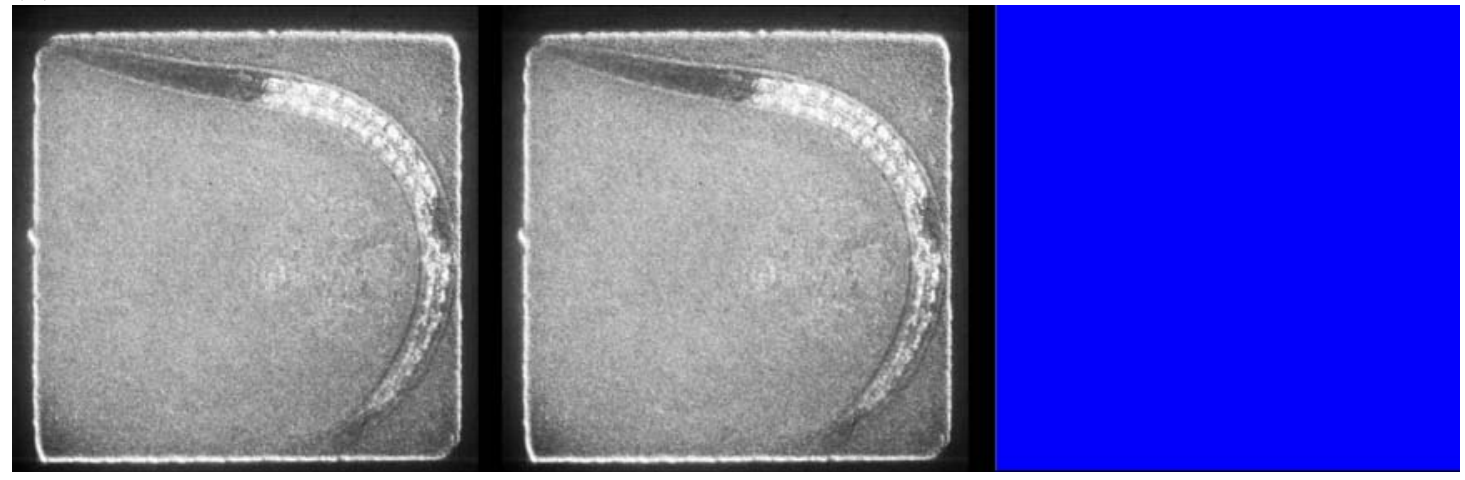

Figure 3.7: Visualization of the principle of sleep detection using image subtraction. Two consecutive frames captured with a $\Delta T$ of $1 \mathrm{~s}$ and their corresponding image subtraction picture during (a) the L1 wake state and (b) the L1 sleep state. Blue corresponds to low, red to high differences between pixel values.

with $k_{s t d}, k_{\text {mean }}$ : empirically determined constants that depend on the local time window observed. For wild-type worms a time window of five frames, $k_{1}=40$ and $k_{2}=1.5$ yields robust detection of the sleep state. Figure 3.8 shows the time course of (a) local standard deviation and (b) local mean of image subtraction values and their respective sleep detection thresholds as indicated by a red line. Using this parameters the Equations 3.1 can be rewritten (Equations 3.2).

$$
\begin{aligned}
\operatorname{std}\left(\operatorname{Imsub}\left(t_{n-4, \ldots, n}\right)\right) & \leq \operatorname{std}\left(\operatorname{Imsub}\left(t_{0, \ldots, n}\right)\right) / 40 \\
\operatorname{mean}\left(\operatorname{Imsub}\left(t_{n-4, \ldots, n}\right)\right) & \leq\left(0.5 * \operatorname{mean}\left(\operatorname{Imsub}\left(t_{0, \ldots, n}\right)\right)+\min \left(\operatorname{Imsub}\left(t_{0, \ldots, n}\right)\right)\right) / 1.5
\end{aligned}
$$

with $t_{0}$ : timepoint of first image subtraction value, $t_{n}$ : last point in time $\operatorname{Imsub}(t)=\sum i j \operatorname{ImSub}(t)_{i, j}$ 
(a)

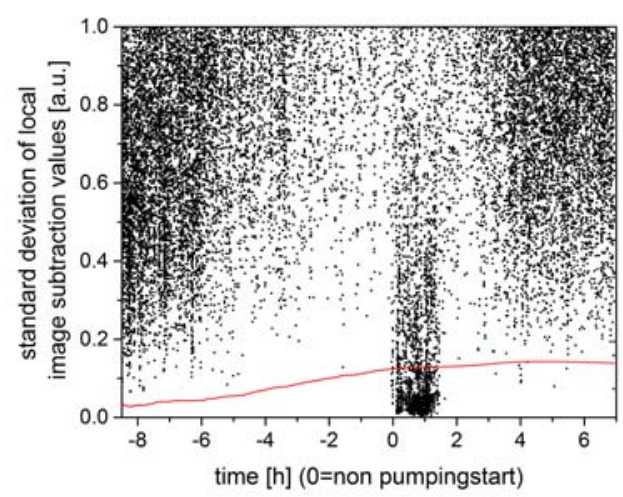

(b)

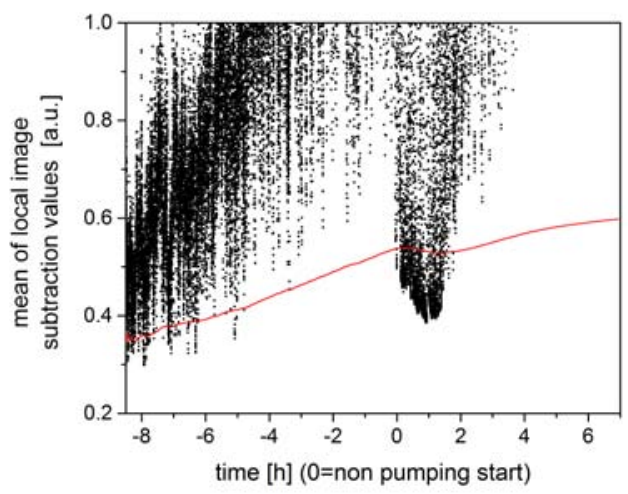

Figure 3.8: Detection of sleep like state is based on the local standard deviation and the local mean of image subtraction values. Shown is the timecourse for (a) the local standard deviation (b) the local mean of image subtraction values for a single animal. The threshold for sleep detection is visualized by a red line.

\subsubsection{Evaluation of sleep detection using the image subtraction method}

I evaluated the sleep detection criterion for 25 animals that were not implicated in the postulation of the sleep detection criterion (Figure 3.9). In $89.3 \%$ of the cases sleep was detected during the non pumping period and allowing a tolerance of $5 \mathrm{~min}$ prior to non pumping start $96.4 \%$ was detected correctly. Sleep was detected on average with a delay of 5.1 minutes after the non pumping start. The implementation of the sleep detection criterion as part of the "C. elegans automated sleep deprivation" program was done in LABVIEW, see section 2.6. 


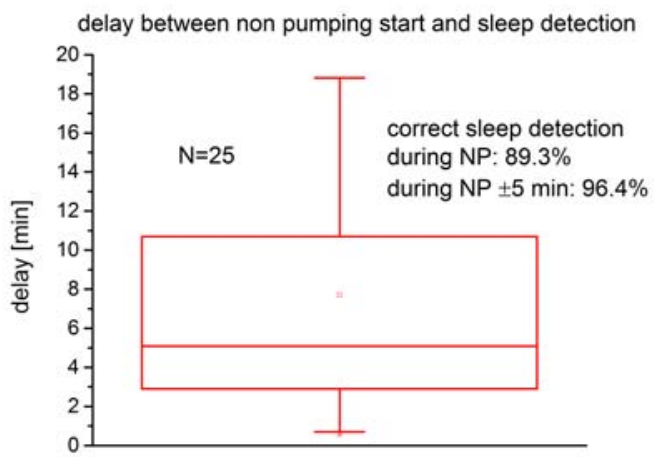

Figure 3.9: Evaluation of the timing and the accuracy of sleep detection. Shown is a boxplot representation of the distribution of sleep detection timepoints during the non pumping period. 
3.2. Automated sleep deprivation in C. elegans - Comparing ChR activation of neurons and mechanical stimulation by dish tapping.

\subsection{Automated sleep deprivation in C. elegans - Comparing ChR activation of neurons and mechanical stimulation by dish tapping.}

Established sleep deprivation methods in C. elegans are manual mechanic stimulation with an eyelash or platinum wire pick and forced swimming (in a constantly vortexed liquid) [36], [84], [41].

My requirement for a sleep deprivation stimulus were non physical harmfulness and high reproducibility. I tested two different methods, ChR2 activation of neurons and mechanical stimulation by dish tapping.

\subsubsection{ChR2 light activation of neurons}

The nematode C. elegans shows a strong avoidance behavior to blue or shorter wavelengths of light, see [85]. The magnitude of the induced velocity is proportional to the incident light intensity and there is a reaction delay of approximately one second. By using blue light illumination of $1 \mathrm{~mW} \mathrm{~mm}^{-2}$ or more sleep deprivation is possible for thirty minutes and longer. However blue light illumination of the required intensity visibly damages the animal physically (Figure 3.10).

(a)

(b)

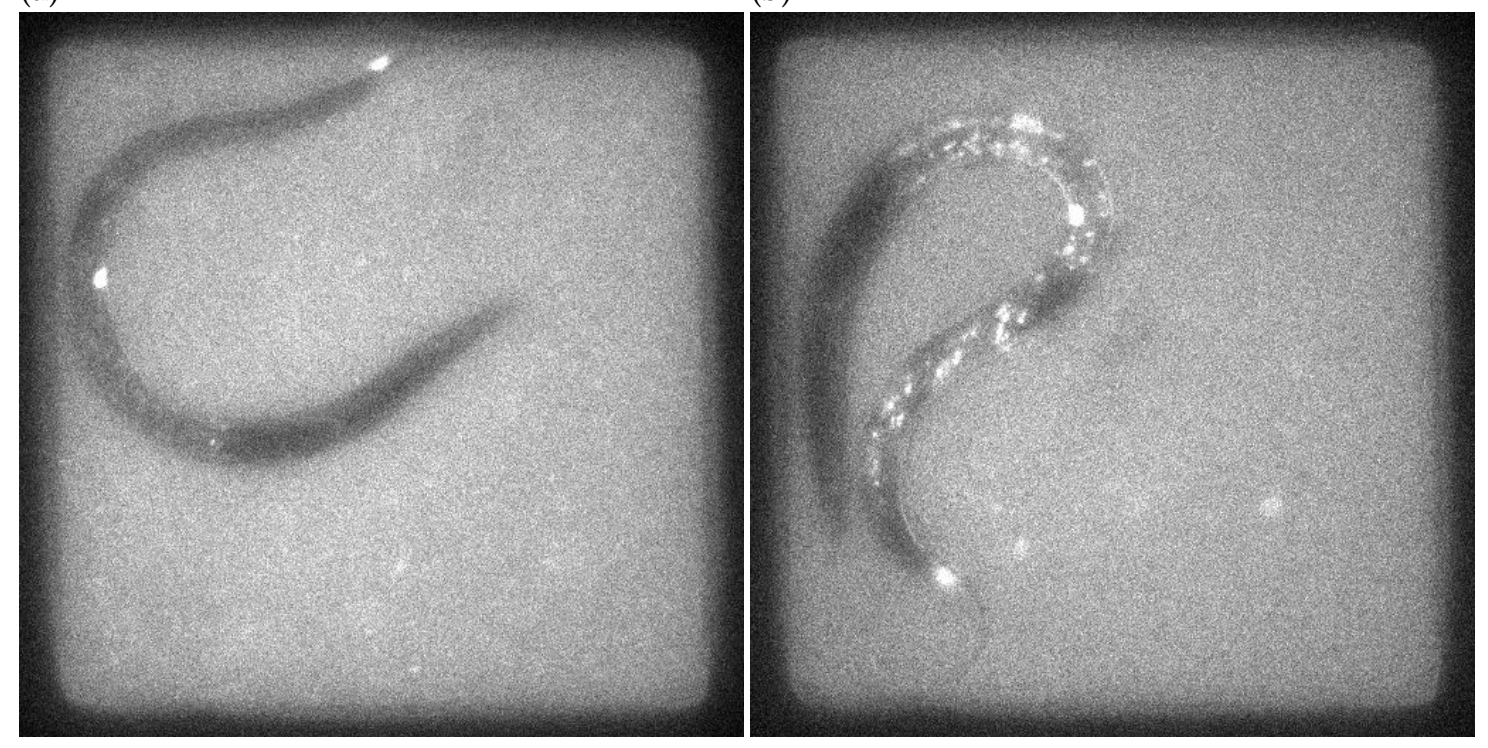

Figure 3.10: Prolonged high intensity blue light illumination causes cellular damage leading to the death of the animal. Cellular damage can be seen by increased fluorescence of $C$. elegans tissue. Fluoerescence image of $C$. elegans (a) prior and (b) past longterm illumination with blue light.

Introducing transgenic light gated channels, so called channelrhodopsins (ChR) to the 
nervous system might allow sleep deprivation with much smaller blue light intensities. Details about the ChR protocols used for the experiments, are described in the methods section 2.5.4. I compared the effect of ChR2 activation in three different subsets of neurons:

- nociceptive sensory neurons: ASH and PVQ - sra-6 promoter (psra-6)

- mechanosensory neurons: ALM, PLM and AVM - mec-4 promoter (pmec-4)

- (motor-) interneurons involved in nociception: AVA, AVD, AVE, AVG, RIM and PVC - nmr-1 promoter (pnmr-1)

For all three subsets of neurons ChR2 activation leads to a mobilization of the animal. Activation of ChR2 expressing in pnmr-1 and pmec-4 leads to a strong but only acute response to low blue light intensities. ChR2 activation of nociceptive sensory neurons (psra-6) is triggering the strongest persistent behavioral response during both sleep and wake (Figure 3.11). The difference between the transgenic lines is probably due to much higher ChR2 expression levels in the psra- 6 transgenic line. psra-6::ChR2 has been created by integration of an extrachromosomal array yielding higher copy numbers of the construct containing plasmid than direct integration by microparticle bombardment, as used to generate pnmr-1::ChR2 and pmec-4::ChR2. Having shown that psra-6::ChR2 transgenic line is best suited for sleep deprivation I investigated which combination of light intensity, stimulus length, inter-stimulus pause, and application timing is most efficient (Figure 3.12).

Light stimuli as short as $5 \mathrm{~ms}$ are sufficient to trigger a behavioral response. Prolonged activation for several seconds with low blue light intensity leads to best deprivation results for a given total amount of incident blue light. Sleep deprivation experiments using ChR2 activation have therefore been conducted with a stimulus consisting of 100 pulses, each lasting $100 \mathrm{~ms}$, a inter-stimulus pause of $10 \mathrm{~ms}$ and an intensity between $0.1 \mathrm{~mW} \mathrm{~mm}^{-2}$ to $0.4 \mathrm{~mW} \mathrm{~mm}^{-2}$. To reduce the blue light intensity needed for sleep deprivation I tested whether the use of a ChR2 variant with longlasting activation yields better results. ChR2(C128S) activation of interneurons involved in light nose touch, expressed under the $g l r-1$ promoter lead to a smaller mobility induction in sleep than ChR2(H134R) activation in sensory neurons for the same amount of incident blue light, data not shown. Therefore I did not pursue the idea to use ChR2(C128S) for sleep deprivation.

In a next step I tested whether blue light ChR2 activation can be used for sleep deprivation. For a direct comparison of the effectiveness of sleep deprivation by the activation of channelrhodopsin expressing neurons to dish tapping I used the a promoter expressed in mechanosensory neurons, that are activated by dish tapping too. Blue light induced ChR2 activation of mechanosensory neurons successfully induced persistent mobility in the animal (Figure 3.13). The mean nose speed during sleep increases by about threefold and roughly $2 / 3$ of the total immobility get deprived.

The mobility inducing effect is due to ChR2 activation and not due to the low intensity blue light. A wild-type control sleep deprived with the same blue light intensity used for ChR2 activation of mec- 4 expressing neurons barely reacts to the light (Figure 3.14. 
3.2. Automated sleep deprivation in C. elegans - Comparing ChR activation of neurons and mechanical stimulation by dish tapping.

(a)

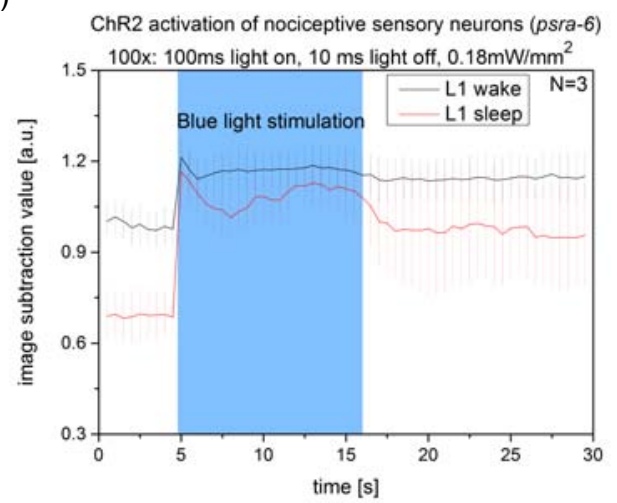

(c)

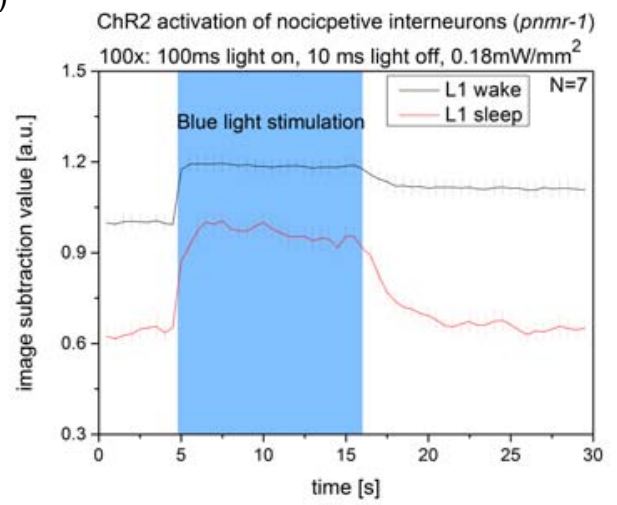

(e)

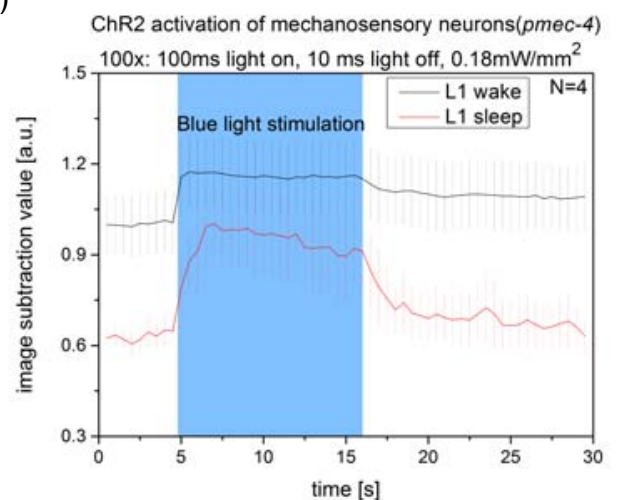

(b)

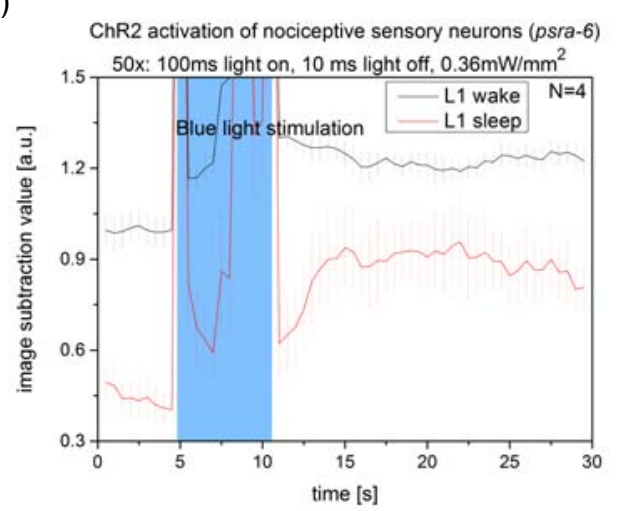

(d)

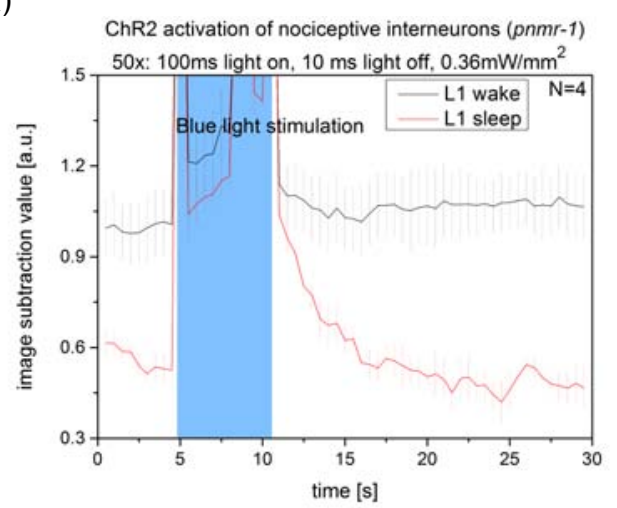

(f)

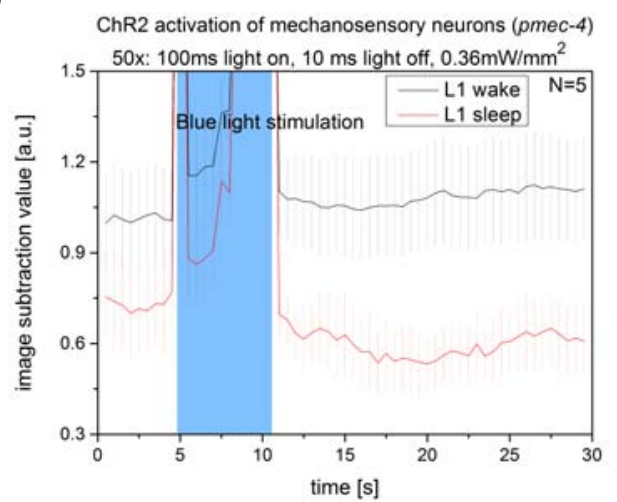

Figure 3.11: ChR2 activation of nociceptive sensory neurons (psra-6) is triggering the strongest behavioral response during both sleep and wake. The wavelength of the incident light was fixed to $490 \mathrm{~nm}$. Stimulus length, interstimulus pause, number of stimuli, and light intensity were varied and are indicated above each figure. (a,b) Nociceptive sensory neurons (psra-6), $(\mathrm{c}, \mathrm{d})$ nociceptive interneurons (pnmr-1) and $(\mathrm{e}, \mathrm{f})$ mechanosensory neurons (pmec-4). The strong increase in image subtraction values for large parts of the blue light illumination period seen for $(b, d, f)$ is an artifact of the blue light shuttering. 
(a)

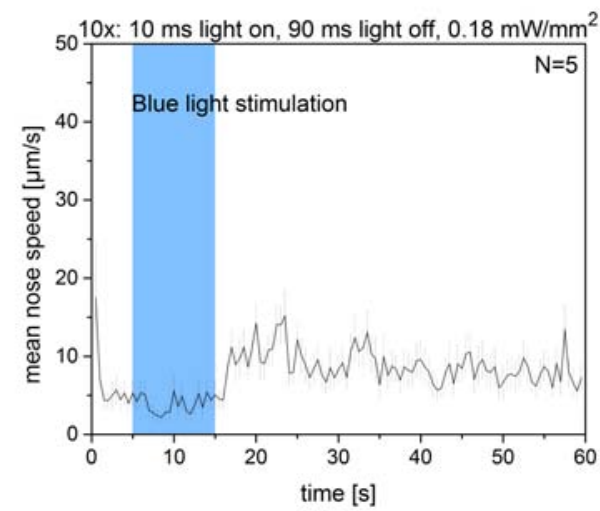

(c)

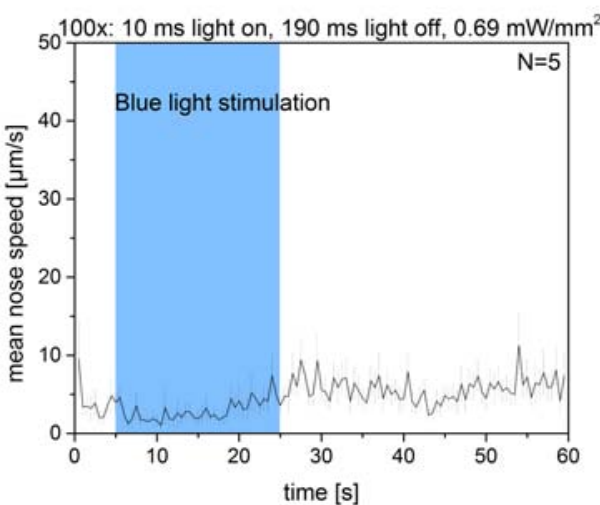

(e)

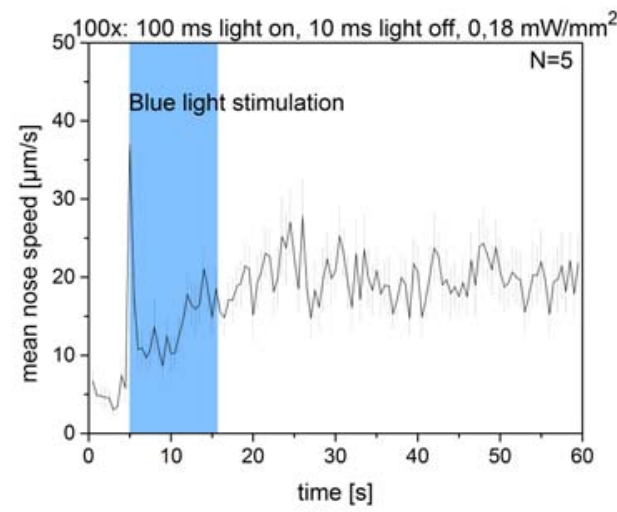

(b)

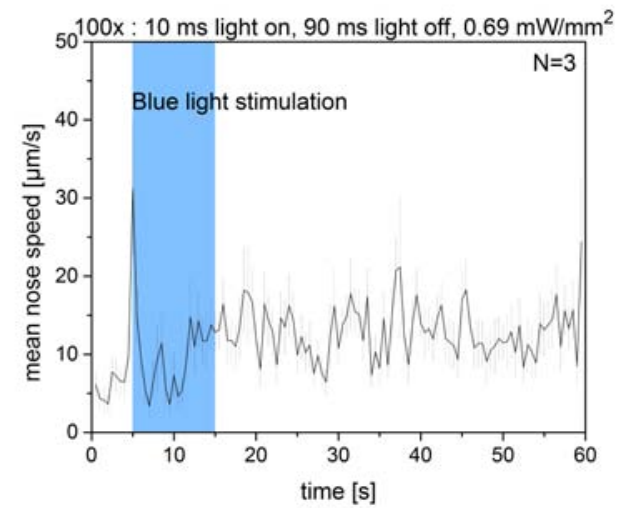

(d)

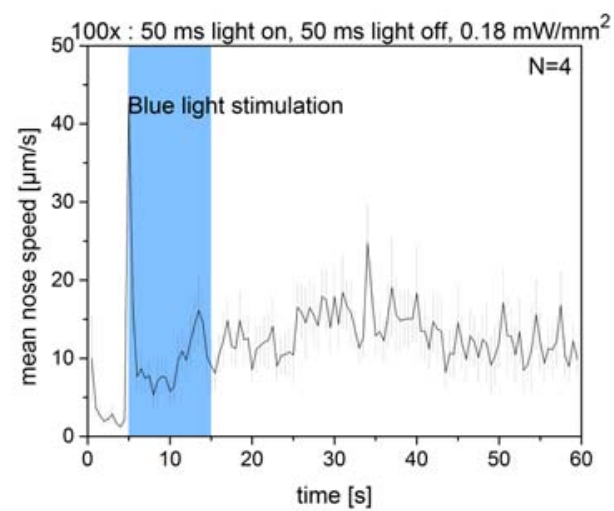

(f)

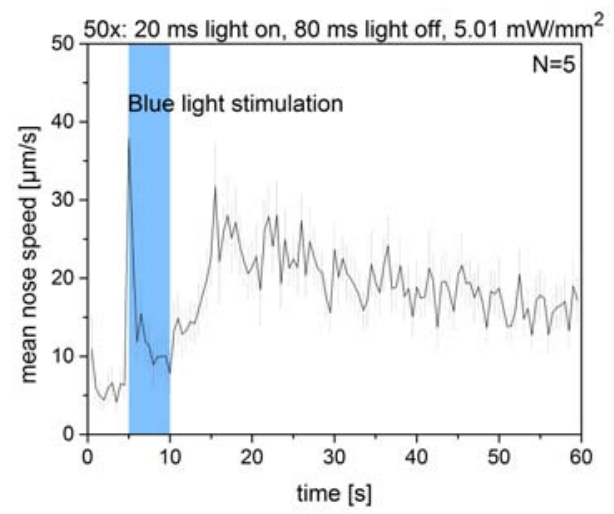


(g)

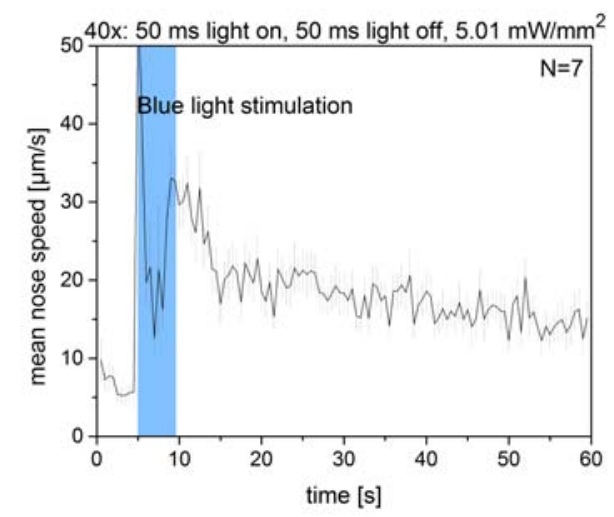

(h)

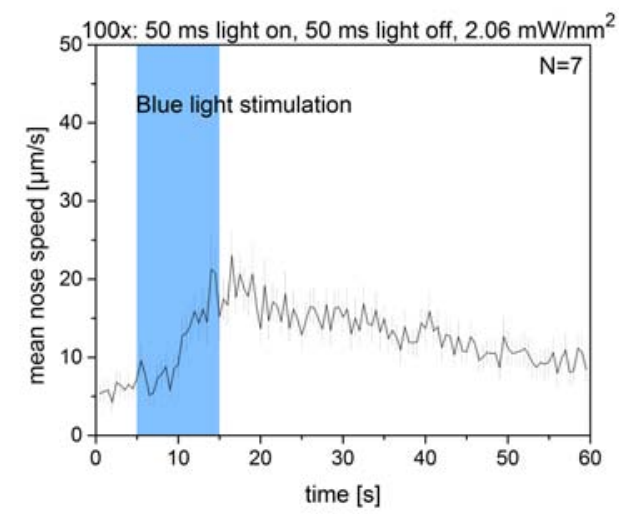

Figure 3.12: Prolonged activation for several seconds with low blue light intensity leads to best deprivation results for a given total amount of incident blue light. (a-h) The wavelength of the incident light was fixed to $490 \mathrm{~nm}$. Number of stimuli, stimulus length, inter-stimulus pause, and light intensity were varied and are indicated above each figure. The strong increase in image subtraction values for parts of the blue light illumination period seen for $(b, d, e, f, g)$ is an artifact of the blue light shuttering.

(a)

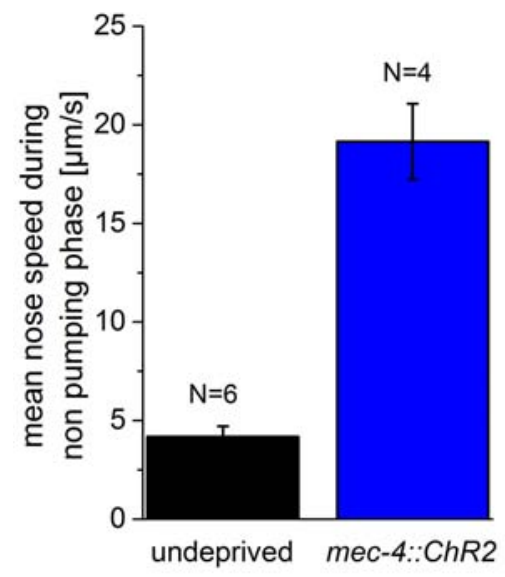

(b)

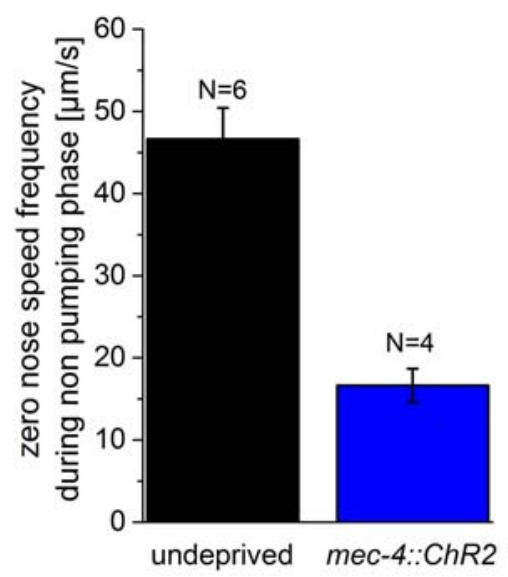

Figure 3.13: ChR2 activation of mec-4 expressing neurons is suited for sleep deprivation in C. elegans. There is a $324 \%$ increase in mean mobility and a $64 \%$ reduction of total immobility. (a) Mean nose speed and (b) zero nose speed frequency for undeprived animals and sleep deprivation by ChR2 activation of mechanosensory neurons. 
(a)

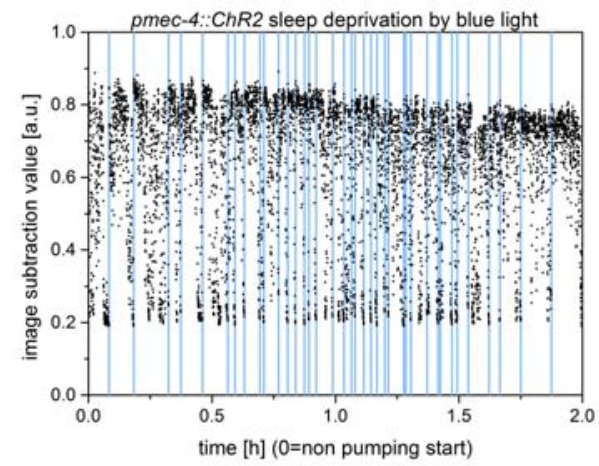

(b)

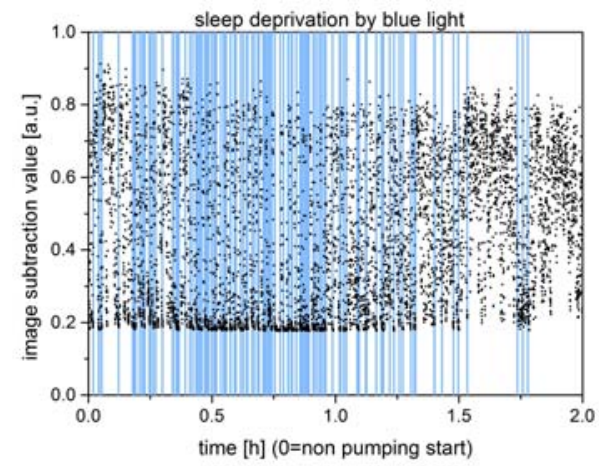

Figure 3.14: ChR2 activation, not blue light activation yields arousal from sleep. Shown is the image subtraction value for the non pumping period for sleep deprivation using $490 \mathrm{~nm}$ blue light, with an intensity of $0.18 \mathrm{~mW} \mathrm{~mm}^{-2}$. Shown is the mobility time course for (a) a transgenic animal $(\mathrm{N}=1)$ expressing $\mathrm{ChR} 2$ under the mec- 4 promoter. For comparison a wild-type control $(\mathrm{N}=1)$ receiving blue light of the same intensity is shown. Light stimulation is indicated by blue shading. 
3.2. Automated sleep deprivation in C. elegans - Comparing ChR activation of neurons and mechanical stimulation by dish tapping.

\subsubsection{Red shifted channelrhodopsin light activation of neurons - C1V1 \& Chrimson}

To bypass the toxic effects and the restrictions for calcium imaging of excitation by blue light, I created transgenic animals expressing C1V1 and Chrimson, both red shifted ChR2 variants [86], [72]. However green light activation of C1V1 did not led to a consistent behavioral response and therefore did not allow efficient sleep deprivation (Figure 3.15). Similarly Chrimson activation of nociceptive sensory neurons (psra-6) and of mechanosensory neurons ( $p m e c-4)$ did not lead to consistent mobility induction in sleep, data not shown. 
(a)

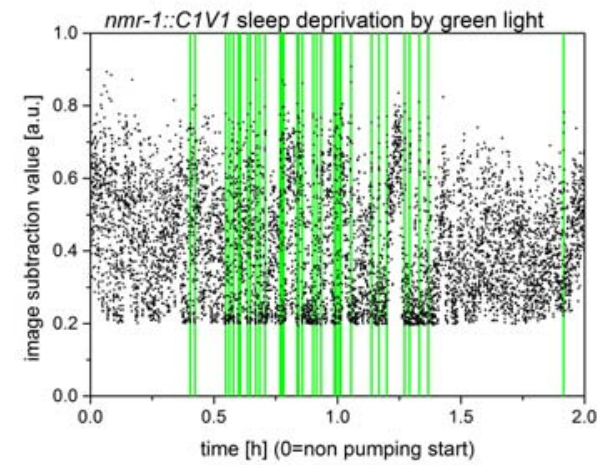

(c)

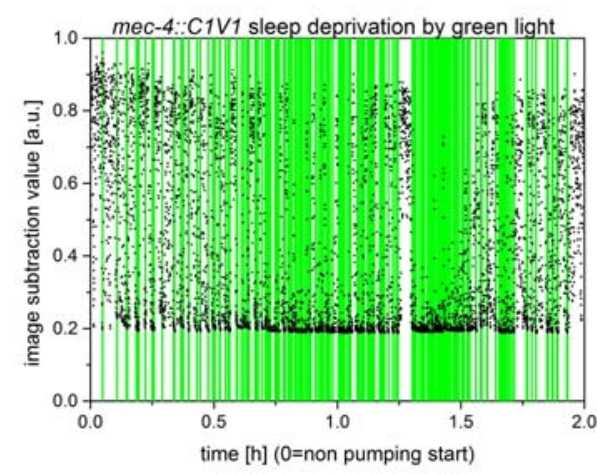

(b)

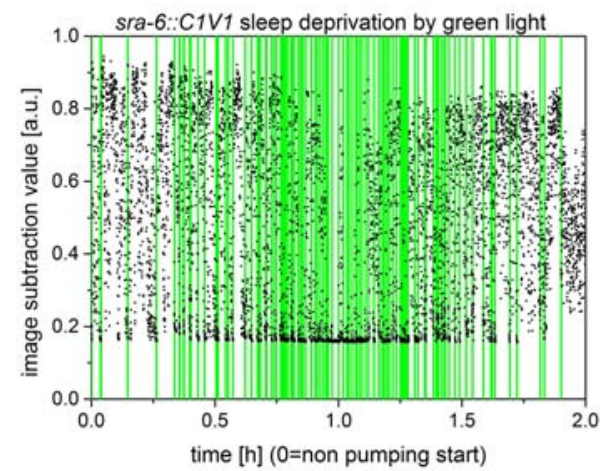

(d)

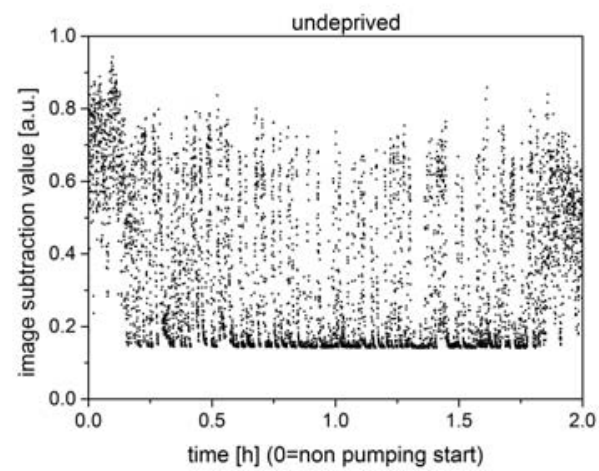

Figure 3.15: C1V1 activation of neurons is not suited for sleep deprivation in C. elegans. Shown is the image subtraction value time course of single animals $(\mathrm{N}=1)$ for the non pumping period. C1V1 was expressed under the following promoters (a) pnmr-1, (b) psra-6 and (c) pmec-4. (d) For comparison an undeprived wild-type control is shown. $(a, b, c)$ For sleep deprivation by C1V1 activation of neurons green light with a wavelength of $595 \mathrm{~nm}$ and an intensity of $0.529 \mathrm{~mW} \mathrm{~mm}^{-2}$ was used. Light stimulation is indicated by green shading.

\subsubsection{Mechanical stimulation by dish tapping}

Details about the dish tapping devices, consisting of a magnet that drives a piston, are described in the methods section 2.5.3. I tested several magnet-piston combinations, that exert different forces on the petri dish containing the nematode. For two of these combinations, that I call mild and strong dish tapping, I investigated if they are suited for sleep deprivation. Mild dish tapping yields a significant mean mobility increase. Compared to undeprived animals sleep deprivation by mild dish tapping induces an increase in nose speed of about $82 \%$ (Figure 3.16a). The total immobility is decreased, 
as indicated by the zero nose speed frequency that drops by about $45 \%$ (Figure 3.16b).

(a)

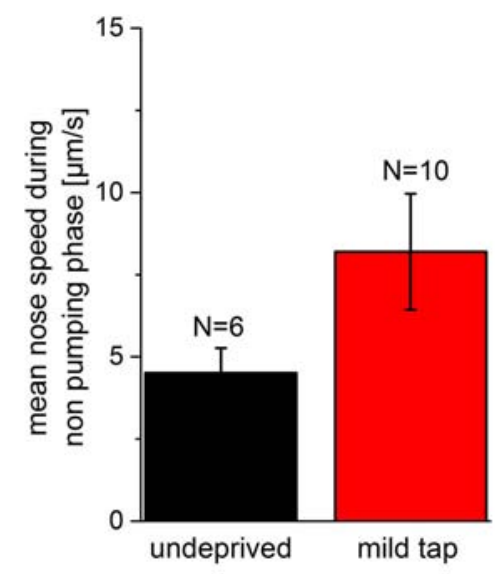

(b)

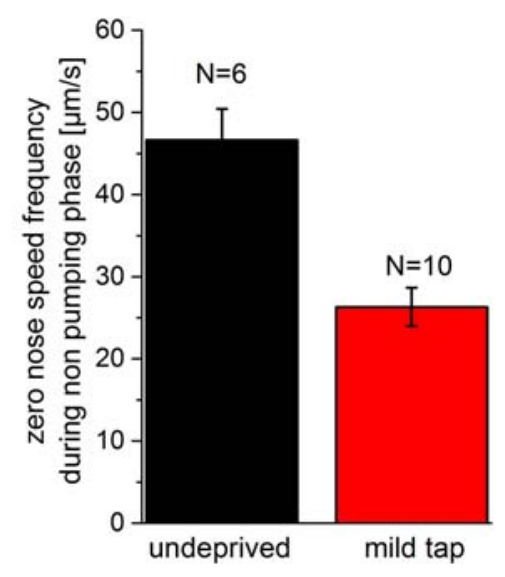

Figure 3.16: Sleep deprivation using mild dish tapping leads to an $82 \%$ increase in mean mobility and to a reduction of total immobility of about $45 \%$. (a) Mean nose speed and (b) zero nose speed frequency for undeprived animals and sleep deprivation by mild dish tapping.

Strong dish tapping leads to even stronger mean mobility increase as nose speed rises about 200\% (Figure 3.16a). Compared to mild tapping less immobility remains, as indicated by the zero nose speed frequency that drops by about $56 \%$ (Figure 3.16b).

Sleep deprivation by strong dish tapping is more efficient than by mild dish tapping, as more immobility is deprived. 
(a)

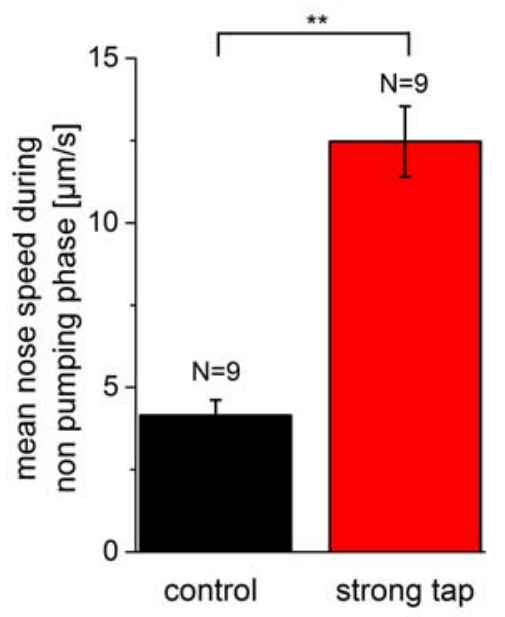

(b)

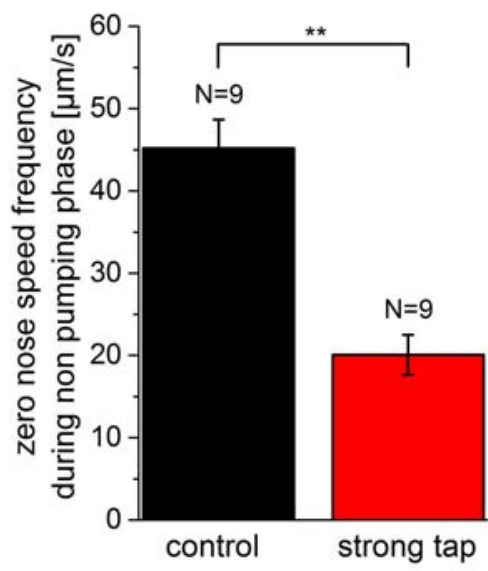

Figure 3.17: Sleep deprivation using strong dish tapping leads to a $200 \%$ increase in mean mobility and to a reduction of total immobility of about $56 \%$. (a) Mean nose speed and (b) zero nose speed frequency for control and sleep deprivation by strong dish tapping. $(a, b)$ The control consists of animals that did receive the tapping stimulus during their L1 wake phase.

\subsubsection{Comparison of deprivation methods}

Sleep deprivation by both $\mathrm{ChR} 2$ activation of neurons and dish tapping can be used for sleep deprivation in C. elegans. Average velocities of sleep deprived animals are comparable to their wake state, but a residual quiescence remains upon sleep deprivation (Figure 3.18). Using blue light activation of ChR2 expressing neurons has two major disadvantages:

First, the longterm blue light illumination is toxic, complicating the analysis of sleep deprivation effects. Second, sleep deprivation can not be combined with independent high resolution calicum imaging like GCaMP because both require blue light excitation. Dish tapping does not have these limitations, as it is non toxic and can be combined without limitations with calcium imaging. Strong dish tapping leads to deprivation of more immobility than mild dish tapping. Therefore I decided to only use strong dish tapping as the sleep deprivation method to investigate on the sleep homeostat. For the sake of simplicity, I will from now on call strong dish tapping just dish tapping. 
(a)

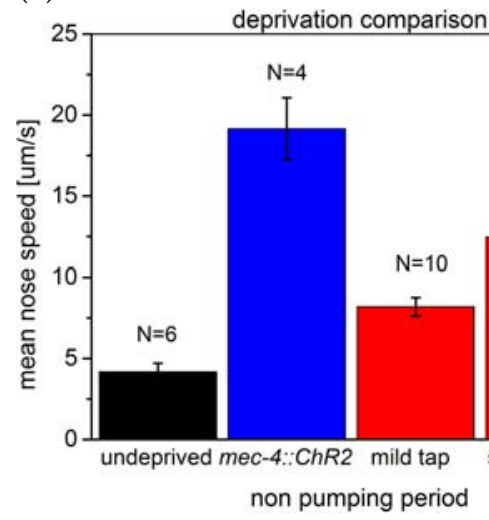

(b)

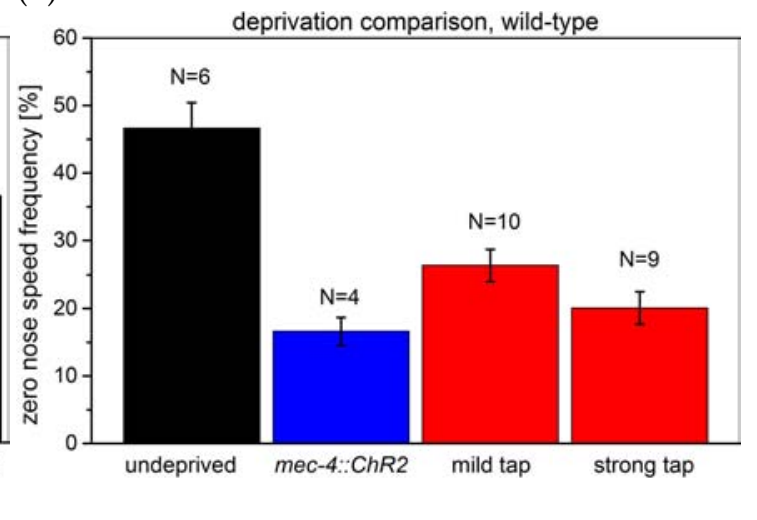

Figure 3.18: Sleep deprivation by both blue light activation of neurons and dish tapping can be used for sleep deprivation in C. elegans (a) Mean nose speed and (b) zero nose speed frequency for undeprived animals, SD by blue light activation of mechanosensory neurons, SD by mild and SD by strong dish tapping. 


\subsection{Sleep deprivation by dish tapping}

The sleep deprivation protocol has been set up in such a way that it roughly deprives half the sleep phase. This allows to measure sleep deprivation effects in the second half of the sleep phase.

\subsubsection{Detailed evaluation of sleep deprivation by dish tapping}

Sleep deprivation by dish tapping induces a strong mobilization of the animal as compared to control animals (Figure 3.19a and 3.19b ). Control animals are filmed together with sleep deprived animals, receiving the mechanical stimulus during their wake phase prior to sleep. The L1 sleep nose speed probability distribution for control and sleep deprived animals illustrates this decrease in immobility (Figure 3.19c). Zero mobility during L1 sleep is reduced by sleep deprivation from $45 \%$ to $20 \%$.

From now on I will distinguish between the period of active sleep deprivation and the consecutive undeprived part of sleep. I compare this sleep deprivation period to the corresponding part of the sleep phase of control animals. Sleep deprivation leads to a $271 \%$ increase in mean nose speed (Figure 3.20a). The average induced velocity of $16 \mathrm{~m} / \mathrm{s}$ is comparable to L1 wake velocities. The time spent immobile decreases by $70 \%$, but a residual quiescence remains upon sleep deprivation (Figure 3.20b). Both, a strong increase in mobility and a strong decrease in immobility are consistently observed for each sleep deprivation-control experiment (Figure 3.20c and3.20d). 
(a)

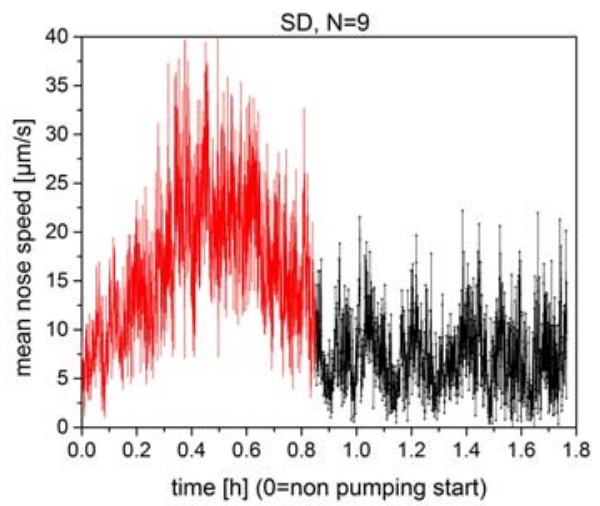

(c)

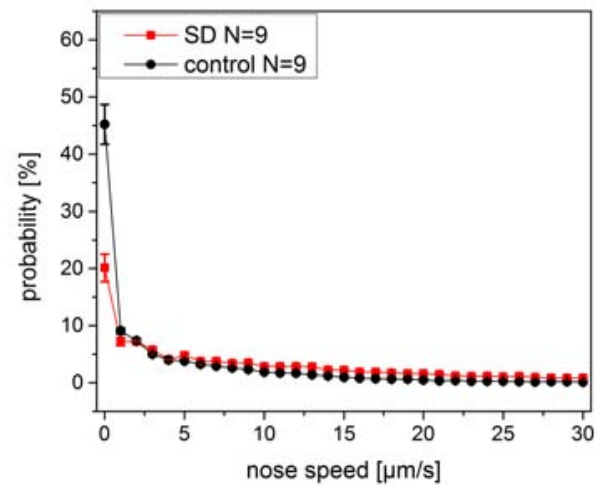

(b)

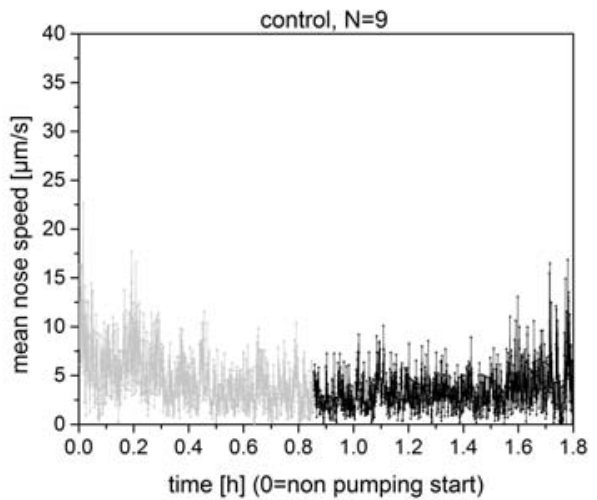

Figure 3.19: Nose speed time course of the L1 sleep phase for (a) sleep deprivation by dish tapping and (b) wake tap control. (c) Probability distribution of nose speed during L1 sleep for sleep deprivation (red) and wake tap control (black). Control animals are filmed together with sleep deprived animals receiving the mechanical stimulus during their wake phase prior to sleep. Sleep deprivation is restricted to the first half of the sleep phase that is indicated by red shading. For control animals the equivalent first segment of the sleep phase is indicated by gray shading. 
(a)

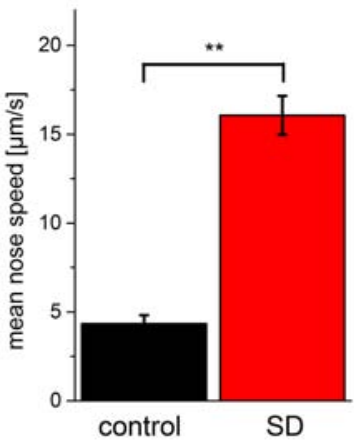

(c)

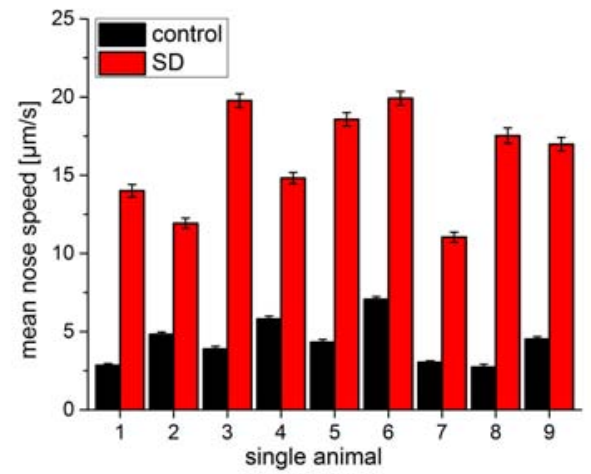

(b)

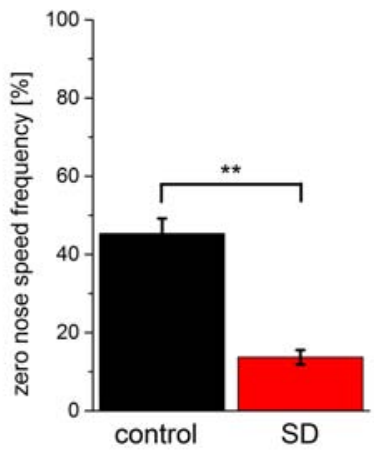

(d)

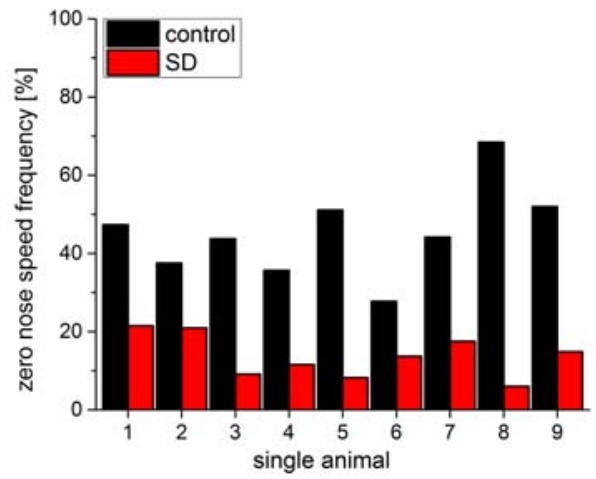

Figure 3.20: Sleep deprivation leads to a $271 \%$ increase in mean mobility and to a reduction of total immobility of about $70 \%$. Shown is the $(a, c)$ mean nose speed and the $(b, d)$ zero nose speed frequency, $(a-b)$ averaged for $N=9$ animals and (c-d) for single animals. Only the deprived part of the sleep phase and the corresponding part of the sleep phase for control animals was evaluated. Control and sleep deprived animals have been recorded simultaneously. Control animals received the tapping during their L1 wake. ** denotes statistical significance with $\mathrm{p}<0.01$, paired Wilcoxon rank test. 


\subsubsection{Effects of sleep deprivation on sleep duration}

To evaluate whether the sleep duration is prolonged upon sleep deprivation I compared the non pumping time of sleep deprived animals with control animals (Figure 3.21a). Surprisingly sleep deprivation shortened the non pumping time by roughly $9 \%$ as compared to control animals. As sleep in C. elegans is interrupted by bouts of mobility, that resemble wakefulness, the total quiescence time might be a more accurate readout for sleep duration. However sleep deprivation also reduced the total quiescence time statistically significantly (Figure 3.21b).

(a)

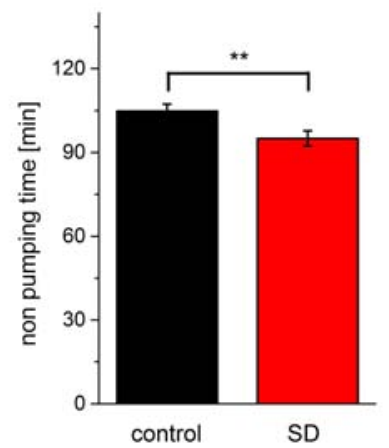

(b)

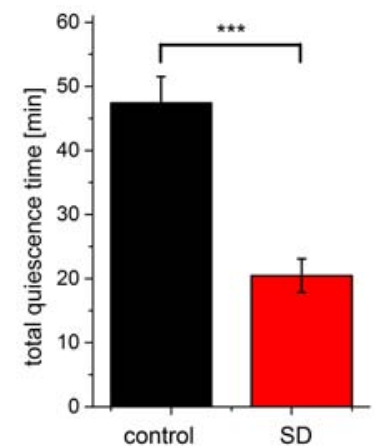

Figure 3.21: Sleep deprivation shortens the sleep duration. (a) Sleep deprived wild-type animals $(\mathrm{N}=9)$ have a non pumping duration that is shortened by $9.34 \%$ compared to control animals ( $\mathrm{N}=9)$. (b) Total quiescence time is decreased by $57 \%$. Control animals received the tapping during their L1 wake. ** denotes statistical significance with $\mathrm{p}<0.01$, paired Wilcoxon rank test.

To investigate whether developmental cues inhibit a rebound in sleep duration I looked at a lin-42 KO mutant. lin-42 is the C. elegans orthologue of period, the gene controlling circadian rhythmicity. This mutation leads to less regular molting timings and to high variations in sleep duration [44]. However sleep deprived lin-42 mutants did not show an increase in sleep length (Figure 3.22). 


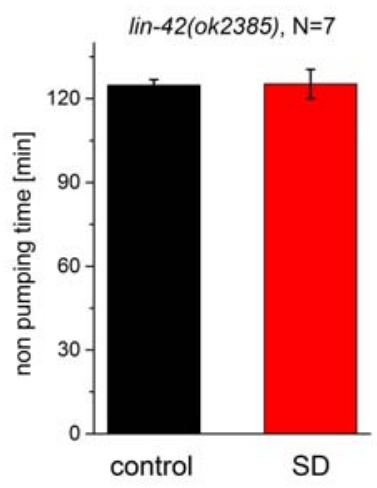

Figure 3.22: Sleep deprivation in lin-42(ok2385) KO mutants does not have an effect on the non pumping duration. Statistical significance was assessed using a paired Wilcoxon rank test.

\subsubsection{Behavioral effects of sleep deprivation}

Having deprived roughly the first half of the sleep phase, I investigated whether sleep intensity is changed during the undeprived second part of the sleep phase. Surprisingly sleep deprived animals are still more mobile than control animals (Figure 3.23).

(a)

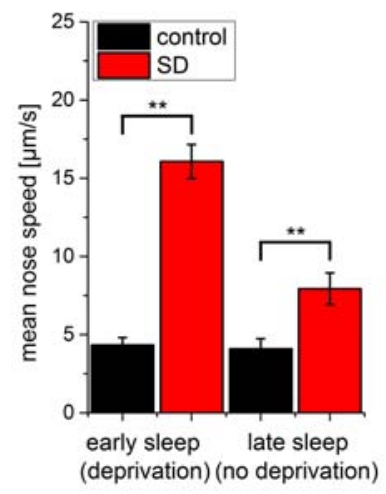

(b)

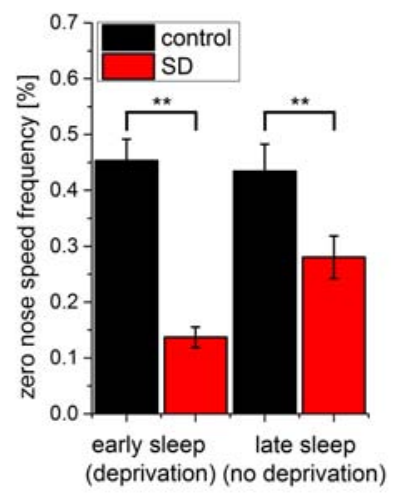

Figure 3.23: Sleep deprivation induces a mobility increase that persists for the undeprived remainder of the sleep phase. (a) Mean mobility and (b) zero nose speed frequency for $\mathrm{N}=9$ animals each. Control animals received the tapping during their L1 wake. ${ }^{* *}$ denotes statistical significance with $\mathrm{p}<0.01$, paired Wilcoxon rank test.

Their mean nose speed is statistically significantly higher and the frequency of zero nose speed significantly lower than for control animals. Extending the sleep depri- 
vation to $70 \%$ of the sleep phase still yields an increase in mean nose speed for the undeprived remainder of the sleep phase (Figure 3.24).

(a)

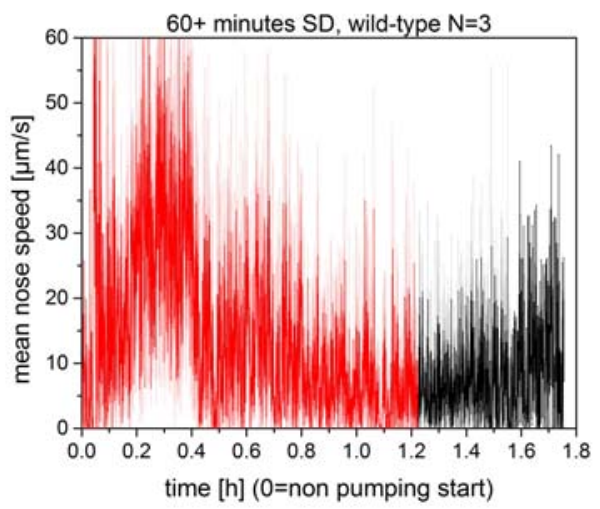

(b)

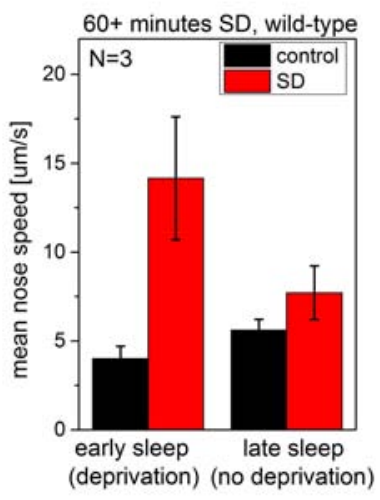

Figure 3.24: Extending sleep deprivation to $70 \%$ of the sleeping phase induces a mobility increase that persists for the undeprived remainder of the sleep phase. (a) Nose speed time course averaged for sleep deprived animals. (b) Mean nose speed during early L1 sleep (sleep deprivation) and late L1 sleep (undeprived sleep) for sleep deprivation and control animals. Control animals received the tapping during their L1 wake.

Behavioral sleep intensity in C. elegans has been assessed priorly by the peak fraction of quiescence and the mean quiescence bout duration [36], the former corresponding to the maximum frequency of immobility for a given time window. Both the peak fraction of quiescence and the quiescence bout duration are decreased following sleep deprivation compared to wake tap control (Figure 3.25). 
(a)

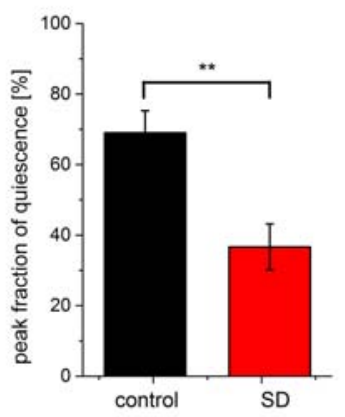

(b)

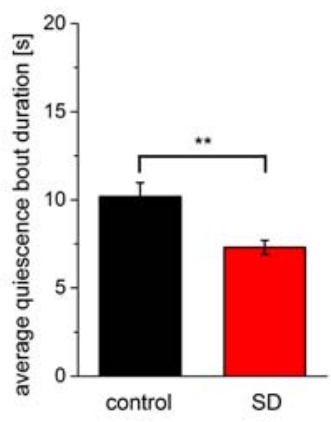

Figure 3.25: Sleep deprived animals left undisturbed in the last part of the sleep phase are still more mobile than control animals. (a) Peak fraction of quiescence and (b) quiescence bout duration for $\mathrm{N}=9$ animals each. Control animals received the tapping during their L1 wake. ${ }^{* *}$ denotes statistical significance with $\mathrm{p}<0.01$, paired Wilcoxon rank test. 


\subsection{RIS activity is reflecting the sleep homeostat}

The gene aptf-1 is required for normal sleep, aptf-1 KO mutants are barely quiescent [40]. aptf-1 is encoding for the AP2 transcription factor that is expressed strongly only in five interneurons in the head, including RIS during early larval development. By generating a transgenic line expressing the calcium indicator GCaMP under the aptf-1 promoter Michal Turek showed that the interneuron RIS is strongly activated prior to and at the sleep onset (Figure 3.26). Optogenetic activation of aptf-1-expressing neurons induced acute, ectopic behavioral quiescence in an aptf-1-dependent manner.

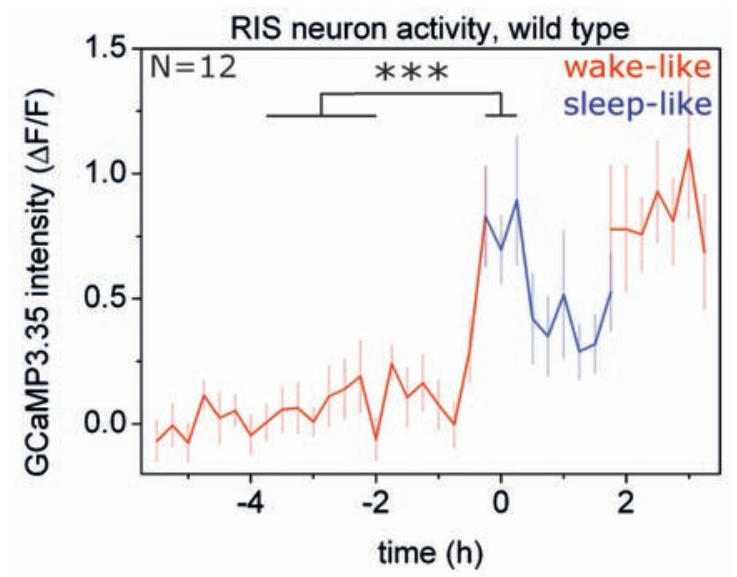

Figure 3.26: RIS GCaMP3.35 activity peaks at sleep onset. Time $=0$ corresponds to non pumping start, taken from [40].

\subsubsection{RIS activity in undeprived wild-type worms}

The fact that RIS is driving quiescence at the sleep onset makes it an interesting candidate for the sleep homeostat. To have a more precise baseline for sleep deprivation experiments I repeated the RIS calcium imaging measurements of undeprived animals with a higher frame rate. As a compromise between high temporal resolution and toxicity effects due to the blue light I chose a frame rate of $0.25 \mathrm{~Hz}$ to record calcium activity. The higher temporal resolution results in a more detailed RIS activity pattern (Figure 3.27a). There is a peak of RIS activity just prior to the non pumping start (time $=0$ ). RIS stays active at a high level for about 20 minutes. The sleep onset RIS peak is followed by several peaks that are narrower and of smaller amplitude (Figure 3.27b). At the end of the non pumping period RIS activity peaks are of higher amplitude and frequency. A remarkable feature of RIS activity is, that it always rises in peaks lasting between 30 seconds and a few minutes.

There is a close relationship between RIS activity and the mobility of the animal. Peaks of RIS activity typically occur after a bout of increased mobility. While RIS activity is high, mobility is low. A reduction in RIS activity is normally accompanied by an 
(a)

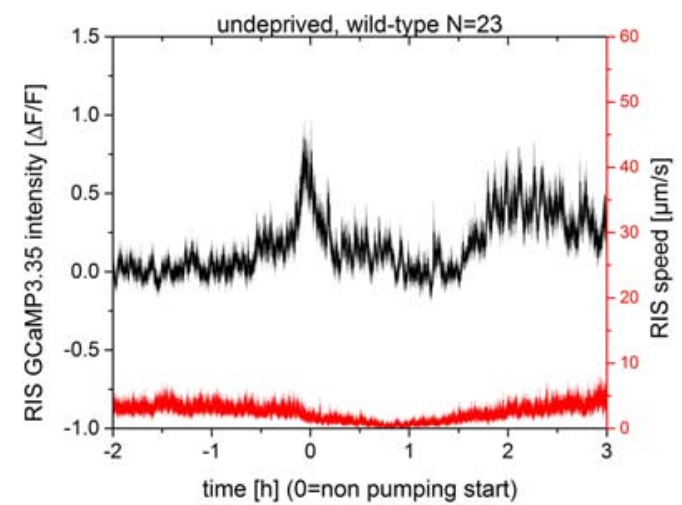

(b)

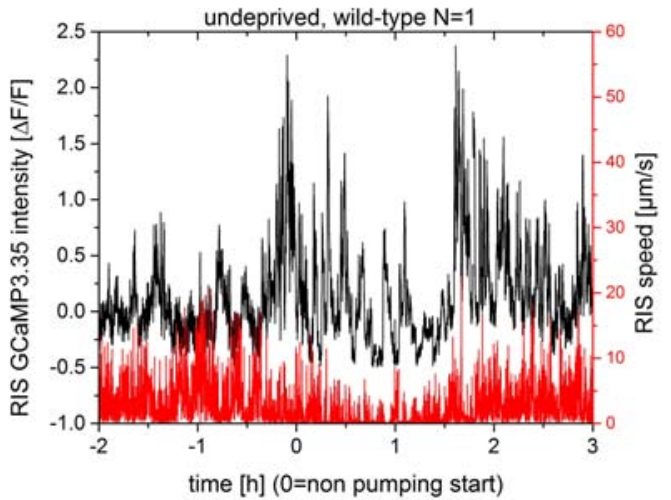

Figure 3.27: RIS-activity and -velocity time course (a) averaged for 23 animals, (b) for a typical single animal.

increase in mobility (Figure 3.28a). To quantify the relation between RIS activity and mobility I created histograms of RIS intensity and velocity (Figure 3.28b, 3.28c, 3.28d). High mobility correlates with low RIS activity, whereas high RIS activity correlates with low mobility. The latter holds especially true for the sleep phase, were highest RIS activity is observed for immobility or very low mobility (Figure 3.28b).

The number and position of RIS peaks varies from animal to animal. This variability can not simply be explained by differences in the mobility of the animals. The fact that peaks of RIS activity are triggered following bouts of high mobility and lead to immobilization are strong indications that RIS is involved in the sleep homeostat. 
(a)

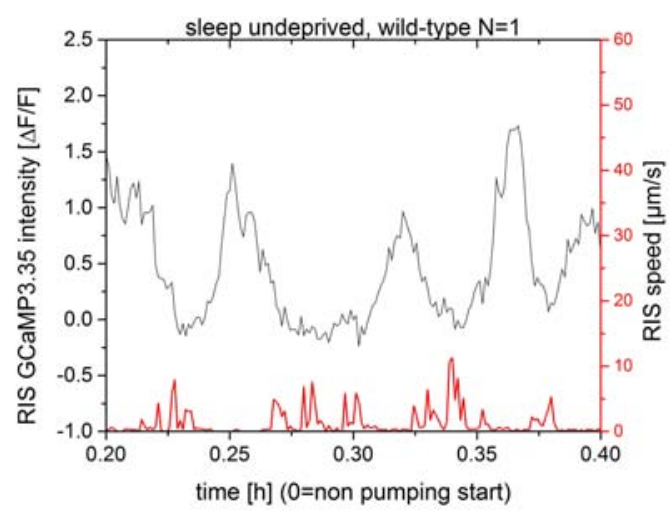

(c)

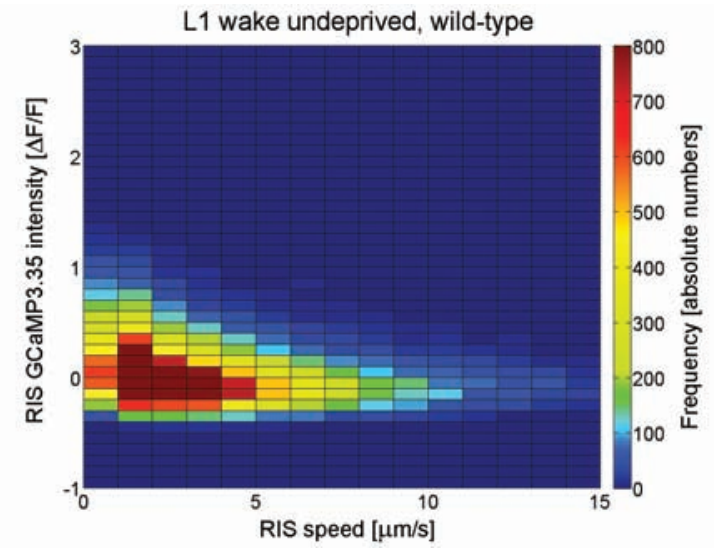

(b)

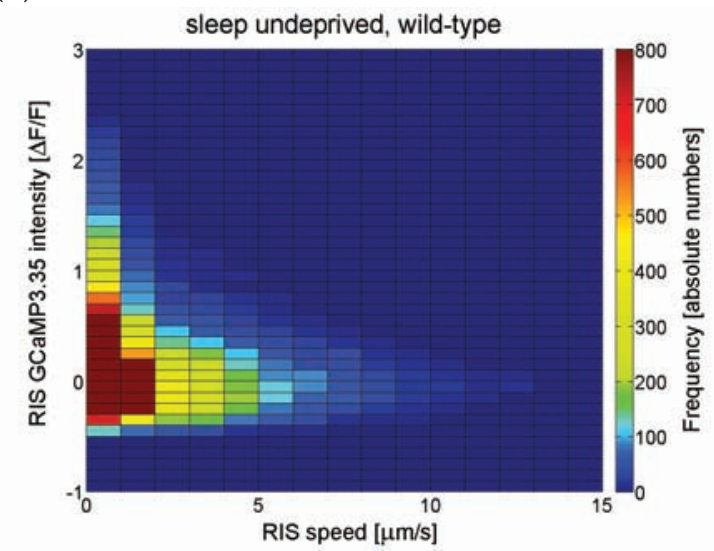

(d)

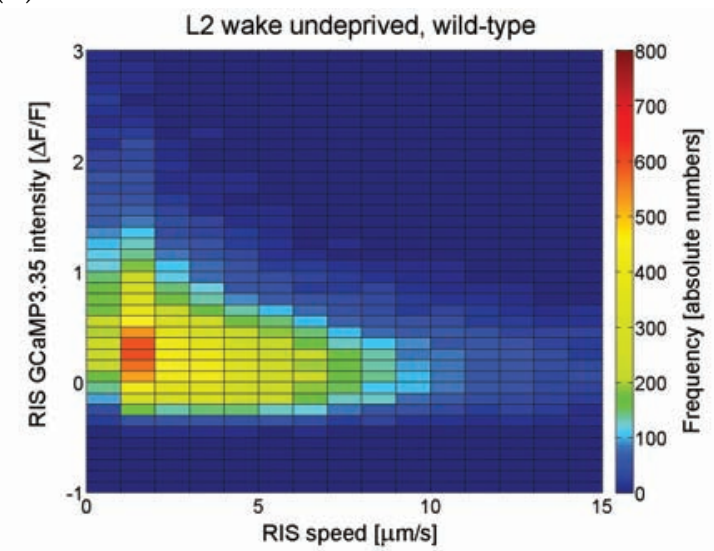

Figure 3.28: Relationship between RIS activity and mobility. (a) Typical RIS activity mobility pattern during sleep. (b-d) RIS activity-velocity histogram for (b) sleep, (c) L1 wake and (d) L2 wake, $N=23$ animals. The color of each square represents the frequency for a given combination of RIS GCaMP intensity and velocity.

\subsubsection{RIS activity upon sleep deprivation}

To investigate homeostatic effects in RIS I sleep deprived transgenic animals expressing GCaMP under the aptf-1 promoter by dish tapping. For stronger homeostatic effects to the deprivation stimulus I used the "continuous sleep deprivation protocol". Figure 3.29a shows the averaged time course of RIS activity and velocity for nine animals that have been sleep deprived from the point when sleep has been detected for the first time. This I call early sleep deprivation, or simply sleep deprivation.

Sleep gets deprived for approximately one hour. Animals are aligned by the start of the non pumping period. Alignment of the animals by the time point when con- 
(a)

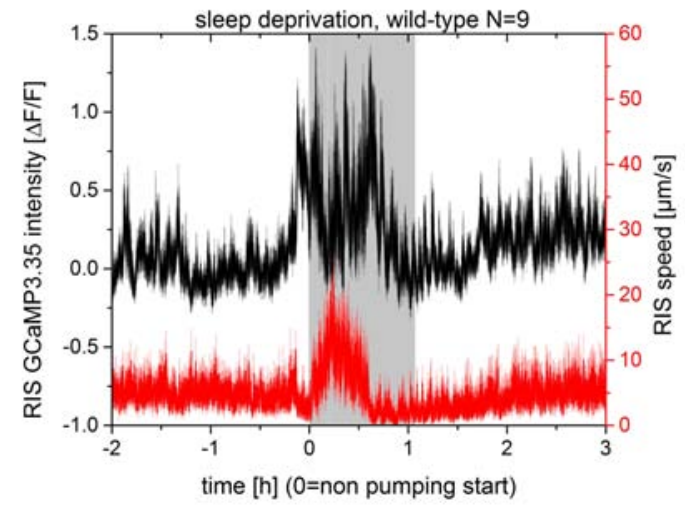

(c)

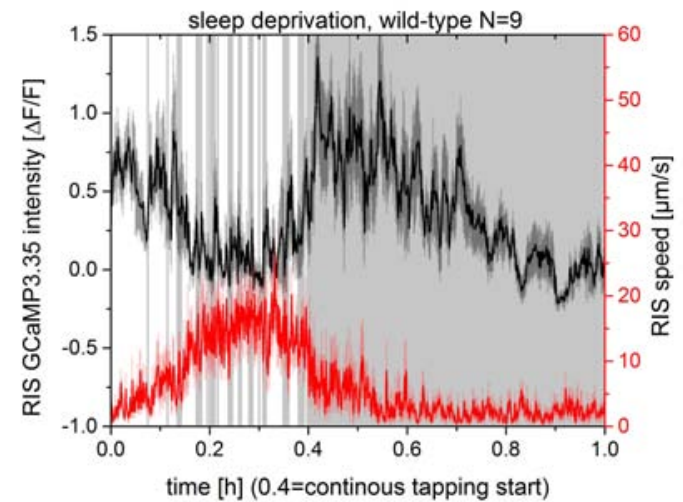

(b)

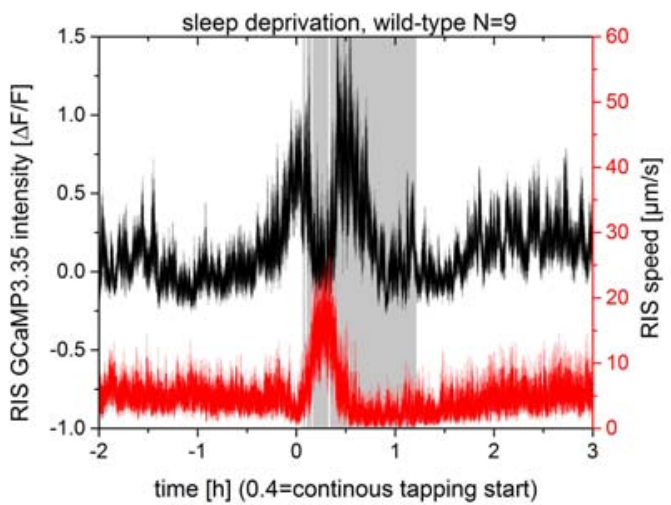

(d)

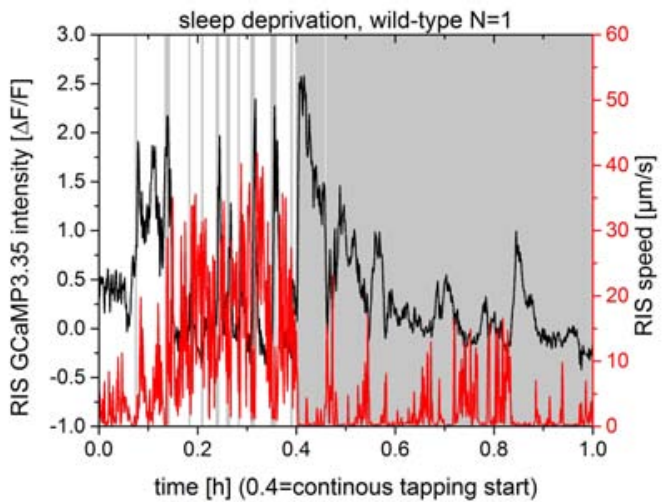

Figure 3.29: RIS activity and velocity time course for (early) sleep deprivation. Average of $\mathrm{N}=9$ animals (a) aligned by non pumping start, (b) aligned by continuous tapping start, (c) close up of (b) and (d) representative time course of a single animal. Dish tapping stimulation is indicated by gray shading.

tinuous tapping starts leads to a sharper and more pronounced peak of RIS activity (Figure 3.29b). The first deprivational tapping stimulus leads to a small increase in RIS activity and to mobilization of the animal (Figure 3.30). The animals show increased mobility in reaction to the tapping stimulus for the first fifteen to twenty-five minutes of sleep deprivation. During this time RIS activity is low and only shows short activation bouts (Figure 3.29c). After fifteen to twenty-five minutes of deprivation, tapping leads to long lasting activation peaks of RIS and to a decrease of the response to the stimulus. Animals immobilize temporarily although they are still exposed to dish tapping (Figure 3.29d). After additional twenty to thirty minutes RIS activity decreases again while the animal still gets stimulated. Now phases of mobility and immobility alternate. At this point smaller peaks of RIS activity are sufficient to immobilize the an- 


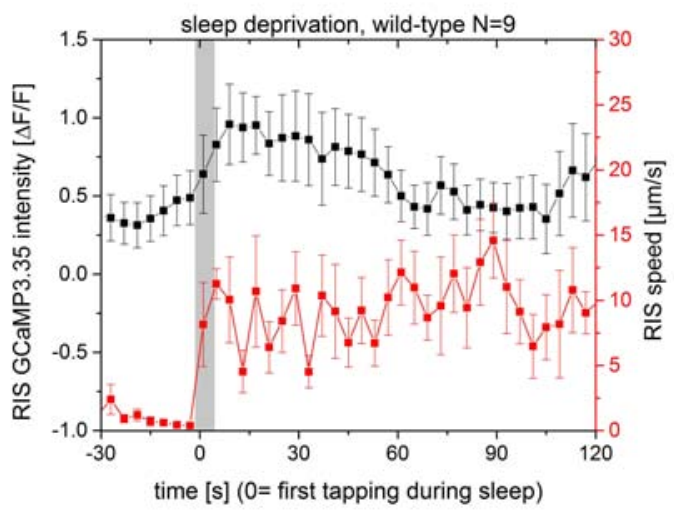

Figure 3.30: RIS activity and velocity response to first deprivational tap stimulus.

imal. RIS activity increases at the transition from sleep to wake similarly to undeprived animals. To sum up: The initial homeostatic response to sleep deprivation is mediated by an induced and long lasting RIS activity peak.

This effect is not to be confounded with habituation, because awake control animals keep on reacting to the stimulus and do not show long lasting RIS activation peaks while being exposed to a similar tapping protocol (Figure 3.31c). Neither is this simply reflecting the depth of the sleep phase. In a protocol where the start of the sleep deprivation is delayed by 30 minutes, which I call late sleep deprivation, the animals show this homeostatic response to the stimulus as well. Although the time course of homeostasis is shorter the deeper the sleep phase (Figure 3.31d).

As a quantitative measure for the time course of homeostasis I applied a linear regression to the slope of decreasing RIS mobility as a reaction to continuous dish tapping: Late sleep deprived animals show the fastest sleep homeostasis with a slope of $-43.21 \mu \mathrm{m} / \mathrm{s}$ per hour, followed by the homeostasis of early sleep deprived animals with $-34.72 \mu \mathrm{m} / \mathrm{s}$ per hour. Wake tap control animals have the slowest homeostasis which has a slope of about $-3.61 \mu \mathrm{m} / \mathrm{s}$ per hour (Figure 3.32). To quantify the amount of RIS activation as a homeostatic reaction to sleep deprivation I calculated the average RIS intensity over an interval of 30 minutes for undeprived control (black), sleep deprivation by tapping during early L1 sleep (red), and late L1 sleep (blue). Figure 3.33a shows these average values for L1 wake, sleep onset, early sleep, late sleep, and L2 wake. By creating the difference between undisturbed control and deprived animals I obtained average evoked RIS intensities (Figure 3.33b). The evoked RIS activity during both early and late L1 sleep deprivation is statistically significantly higher compared to the control condition.

In a similar way I compare the average RIS intensities for the tapping control during L1 wake with those for sleep deprivation. Figure 3.34 shows the average evoked RIS intensity over an interval of 30 minutes for stimulation by tapping during L1 wake (black) and early L1 sleep (red). For both conditions intensity values of undisturbed control animals averaged over the same time window during development have been 
(a)

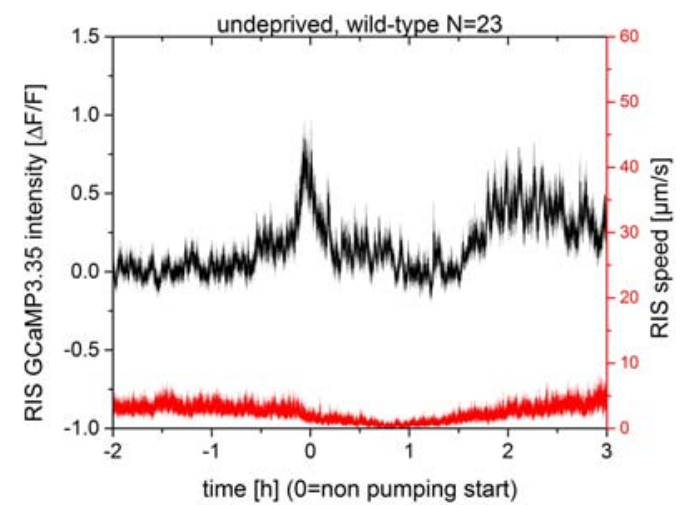

(c)

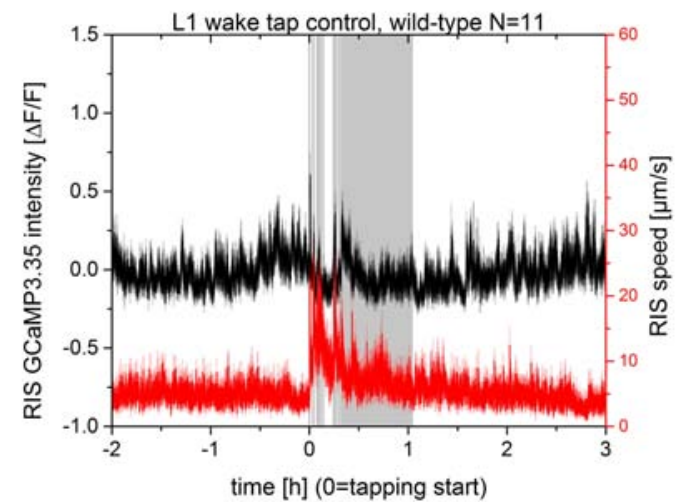

(b)

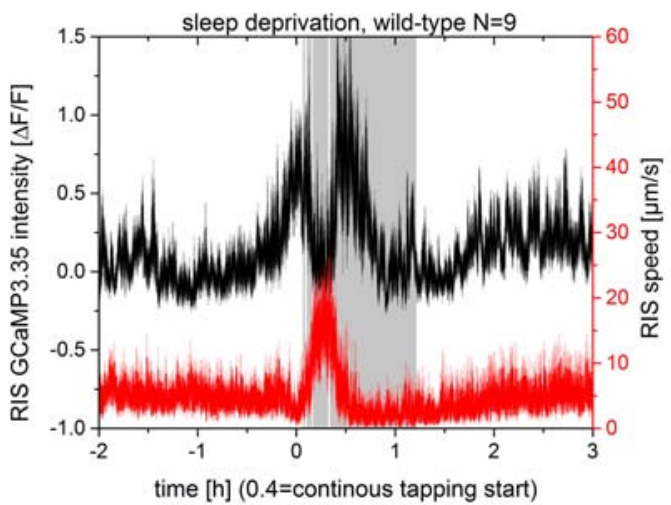

(d)

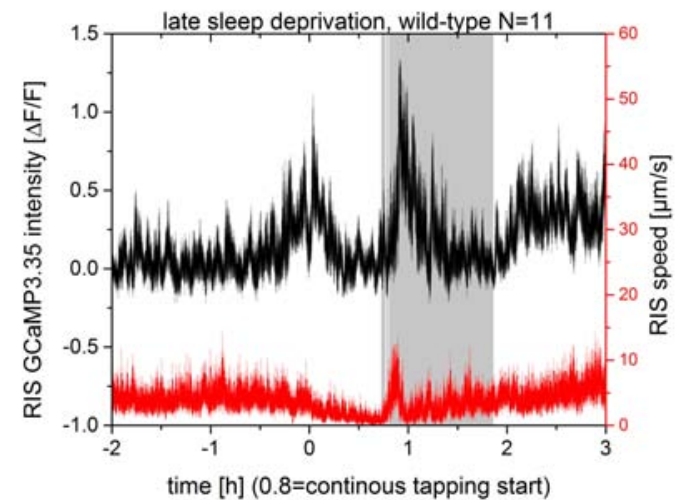

Figure 3.31: Tapping induced RIS activity peak occurs in L1 sleep but not in L1 wake. RIS activity and velocity time course for (a) undeprived control, (b) early sleep deprivation, (c) wake tap control and (d) late sleep deprivation. Dish tapping stimulation is indicated by gray shading.

subtracted. The evoked RIS activity during L1 sleep is significantly higher than for the L1 wake. 
(a)

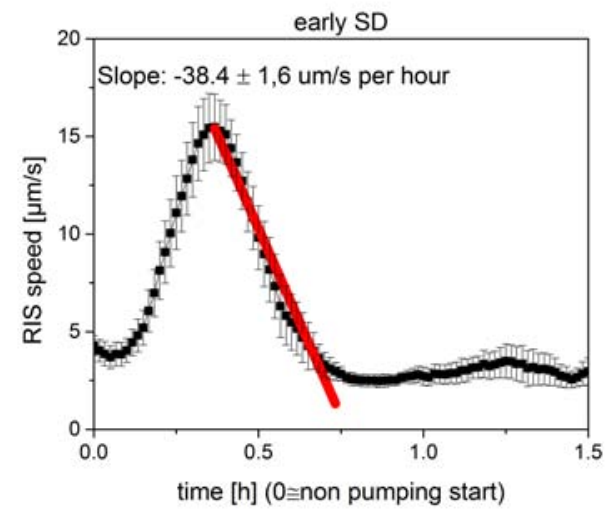

(c)

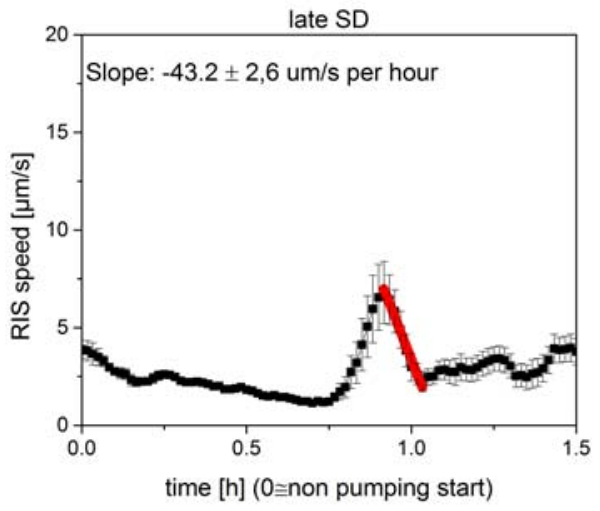

(b)

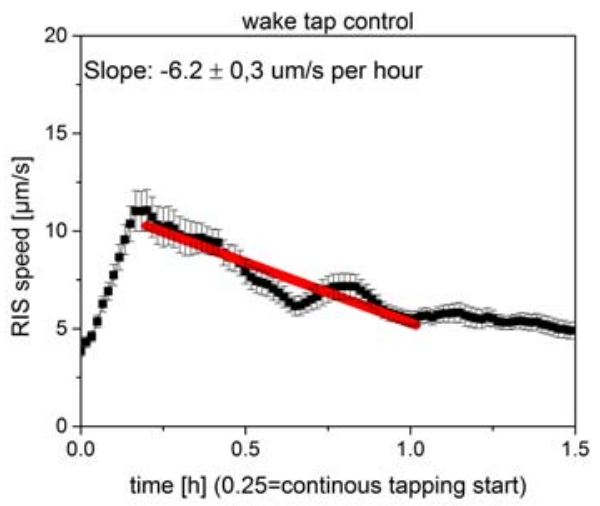

Figure 3.32: The time course of homeostasis/habituation to tapping is dependent on the state of the animal. Shown is the averaged RIS mobility time course for (a) early sleep deprivation ( $\mathrm{N}=9$ ): slope $=-38.4 \mu \mathrm{m} / \mathrm{s}$ per hour, $(\mathrm{b})$ late sleep deprivation $(\mathrm{N}=11)$ : slope $=-43.2 \mu \mathrm{m} / \mathrm{s}$ per hour, $(\mathrm{c})$ wake tap control $(\mathrm{N}=11)$ : slope $=-6.2 \mu \mathrm{m} / \mathrm{s}$ per hour. Each datapoint corresponds to the mean RIS speed over an interval of 15 minutes. The slope of decreasing mobility in response to persistent sleep deprivation was determined using the linear regression fit of ORIGIN and is indicated by a red line. 
(a)

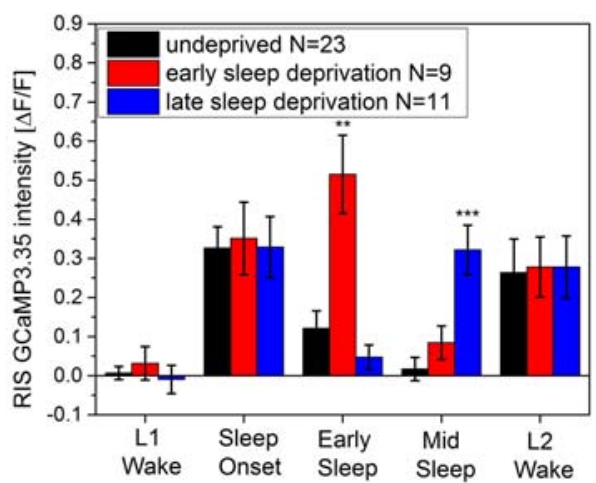

(b)

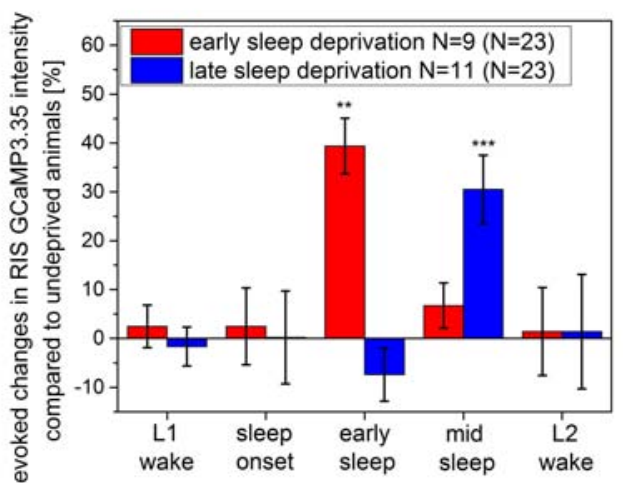

Figure 3.33: Sleep deprivation induces a long lasting RIS activation peak. (a) Shown is the average RIS GCaMP intensity over a period of 30 minutes for undeprived control (black), stimulation by tapping during early L1 sleep (red) and mid L1 sleep (blue). (b) Average evoked RIS intensity over an interval of 30 minutes for stimulation by tapping during early L1 sleep (black) and mid L1 sleep (red). For both conditions intensity values of undisturbed control animals averaged over the same time window during development have been subtracted. The evoked RIS activity for both early and mid L1 sleep stimulation is significantly higher than for the undeprived control animals. ${ }^{* *}$ denotes statistical significance with $\mathrm{p}<0.01,{ }^{* * *}$ denotes statistical significance with $\mathrm{p}<0.001$, Welch test. 


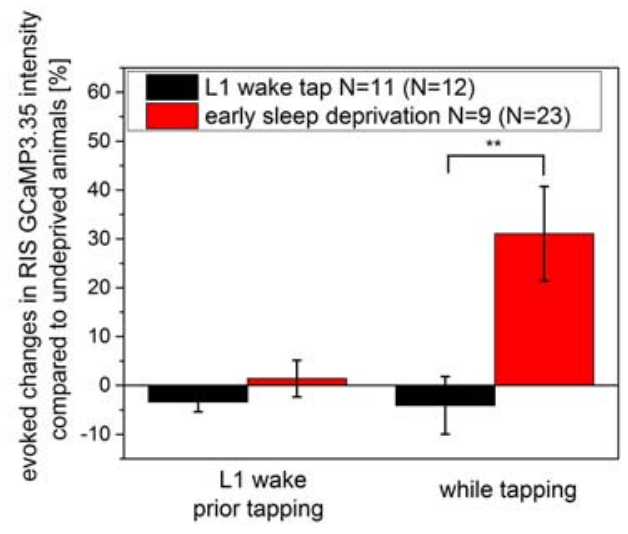

Figure 3.34: RIS activation in response to tapping is dependent on the sleep-wake state: Shown is the average evoked RIS intensity over an interval of 30 minutes for stimulation by tapping during L1 wake (black) and early L1 sleep (red). For both conditions intensity values of undisturbed control animals averaged over the same time window during development have been subtracted. The number of control animals is indicated inside the parentheses. The evoked RIS activity during L1 sleep is significantly higher than for the L1 wake. ${ }^{* *}$ denotes statistical significance with $\mathrm{p}<0.01$, Welch test. 


\subsubsection{RIS activity during sleep following persistent stimulation during L1 wake}

Persistent stimulation of worms prior to their sleep phase might effect consecutive RIS activity during sleep. To maximize the disturbance I applied a sleep deprivation dish tapping protocol three times for 1 hour during L1 wake. Worms that have been persistently stimulated during wake show a RIS sleep onset peak, followed by a reduction of RIS activity during the sleep phase and a rise of RIS at the end of sleep (Figure 3.35). Compared to undeprived animals there is a slight increase of RIS activity during sleep, but this increase is not significant (Figure 3.36).

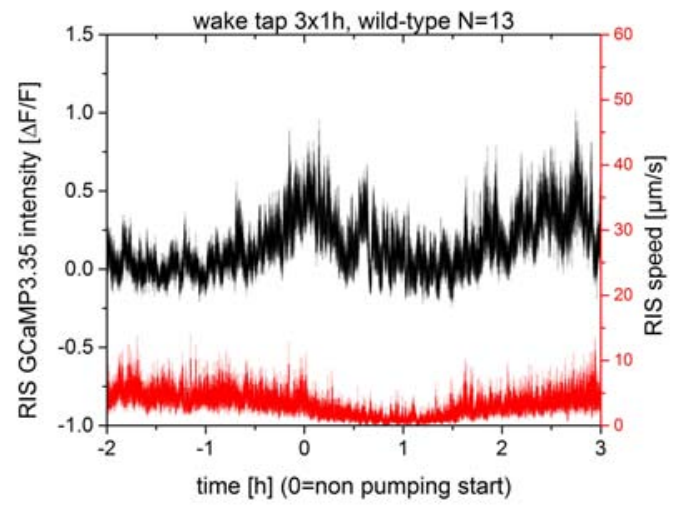

Figure 3.35: RIS activity and velocity during sleep following a persistent tapping protocol during L1 wake. Amount of tapping stimulation corresponds to three sleep deprivation protocols interrupted by two one hour breaks. 
(a)

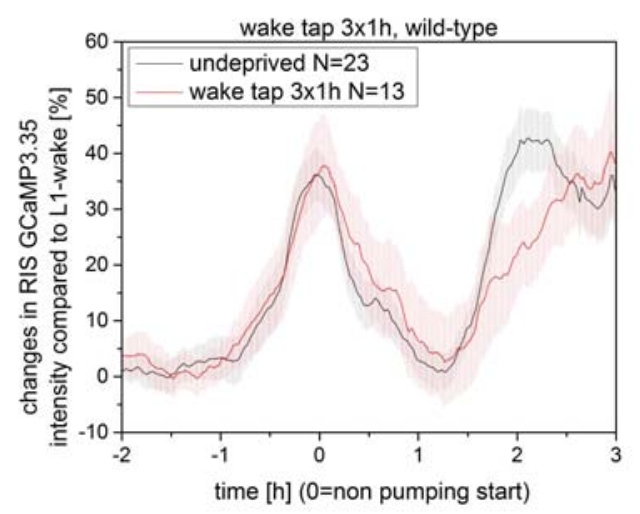

(b)

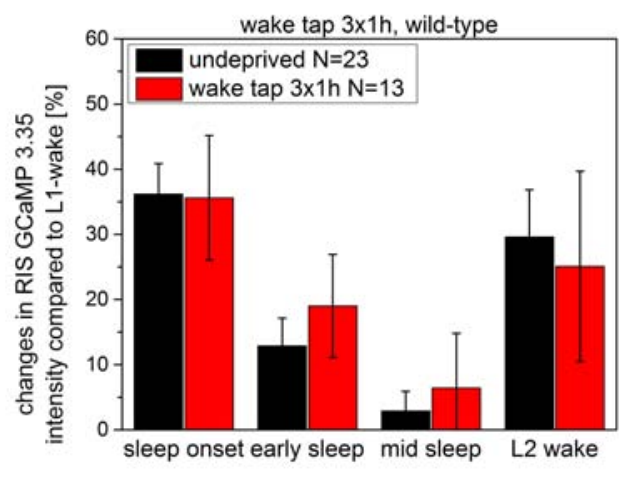

Figure 3.36: RIS activity following three times one hour tapping stimulation prior to sleep is slightly but not significantly increased compared to undeprived worms. (a) Average RIS activity time course following three times one hour tapping stimulation. Each time point represents RIS activity averaged over 30 minutes. (b) Average RIS activity over an interval of 30 minutes for different time points of (a). Statistical significance was assessed using a Welch test. 


\subsection{The sleep homeostat and mechanosensory neuron activity}

To understand how the sleep homeostat works on the sensory neuron level I investigated the activity of the mechanosensory neurons ALM and PLM.

\subsubsection{ALM sensory neuron responsiveness is reduced by prolonged sleep deprivation}

Initially, while the animal strongly reacts to dish tapping, ALM activity rises about $150 \%$ to $300 \%$ above basal levels (Figure 3.37a). With ongoing deprivation time induced ALM-activity continously decreases but still remains elevated compared to basal levels. Awake control animals exposed to a similar tapping protocol show the following ALM activity and velocity time course (Figure 3.37b). ALM activation is strongest at the beginning of dish tapping and decreases to a still higher than basal level. Unlike for sleep deprivation, increased mobility is induced for the whole time of stimulation.

(a)

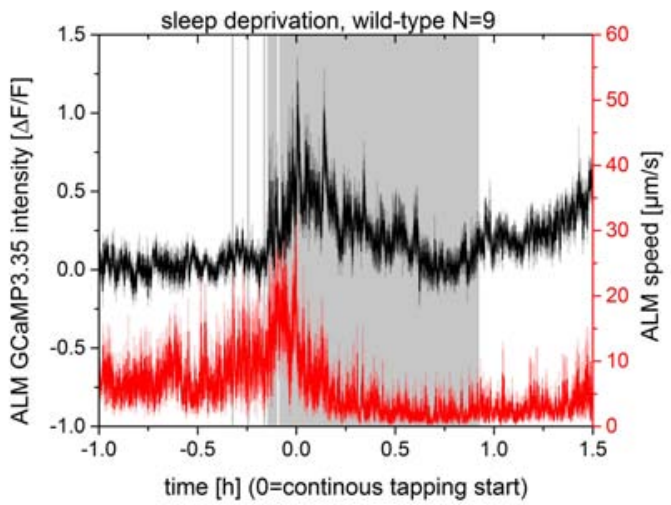

(c)

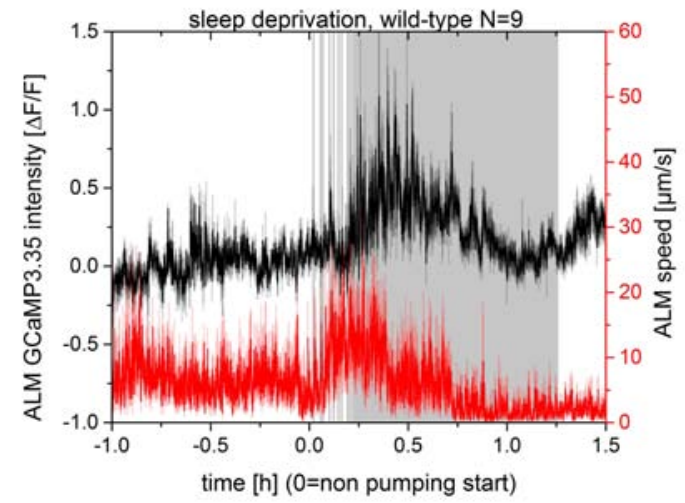

(b)

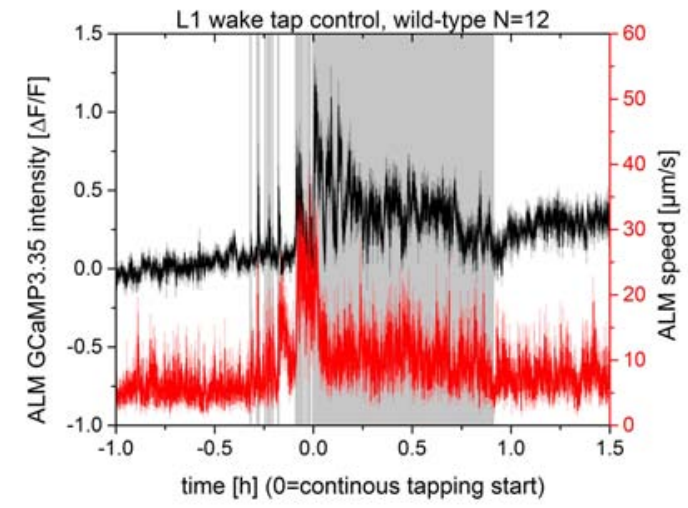

(d)

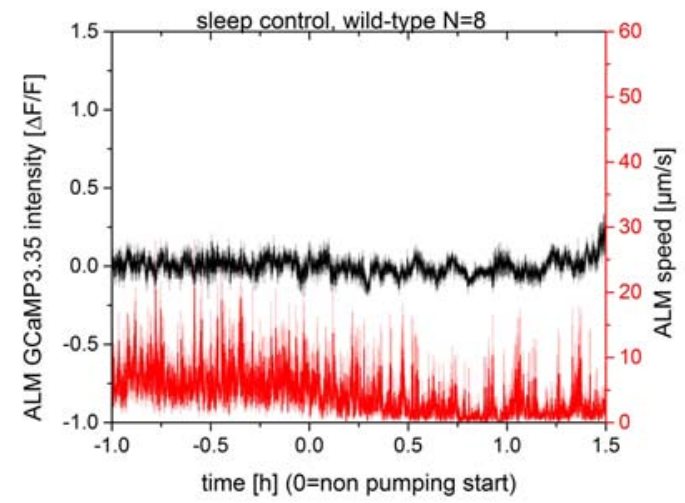


(e)

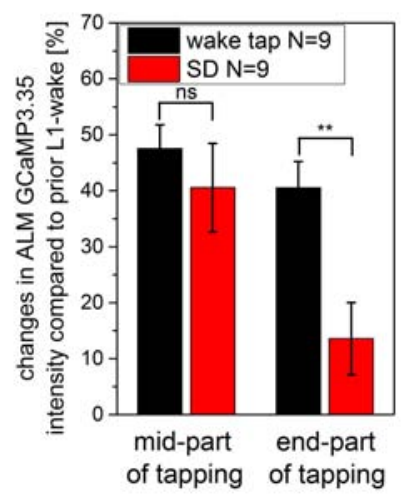

(f)

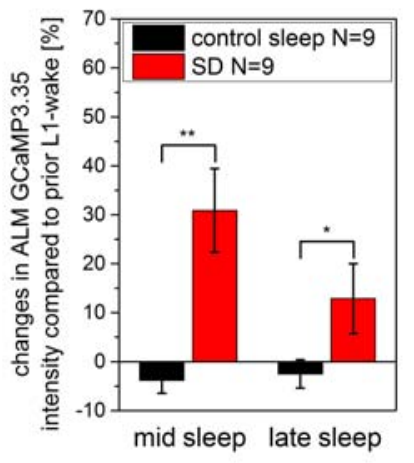

Figure 3.37: ALM sensory neuron responsiveness is reduced during prolonged sleep deprivation. ALM activity and velocity time course comparison for (a) sleep deprivation and (b) wake tap control aligned by continuous tapping start. (c) Sleep deprivation aligned by non pumping start and (d) a undeprived control. Dish tapping stimulation is indicated by gray shading. (e-f) Shown is the average evoked ALM intensity over 21 minutes for the mid and late time period of stimulation/sleep. As baseline, ALM intensity values thirty minutes prior to the stimulation/sleep have been subtracted. (e) Wake tapping control (black) and sleep deprivation (red) aligned by continuous tapping. (f) Undeprived sleep control (black) and sleep deprivation (red) aligned by non pumping start.

Comparing the wake tap control animals and sleep deprivation animals they initially show similar evoked ALM activation patterns (Figure 3.37e). For the last part of the tapping stimulation, ALM activation for sleep deprived animals is significantly lower than for the wake tap control. This means that after prolonged sleep deprivation tapping evoked ALM activation is limited by the sleep homeostat.

For undeprived control animals receiving the tapping protocol during mid-L1 wake, ALM activity slightly decreases during L1 sleep but does not show distinct peaks (Figure 3.37d). Sleep deprivation induces a significant increase in ALM activity for the entire deprivation period compared to undeprived control (Figure 3.37f). To compare undeprived control animals with sleep deprived animals these have been aligned by non pumping start as well (Figure 3.37c).

\subsubsection{The sleep homeostat drives quiescence while mechanosensory neurons still sense the deprivation stimulus}

An interesting time point is the early phase of sleep homeostasis. ALM activity at this time is still largely elevated compared to basal levels (Figure 3.37a). A typical single animal time course of ALM activity vs. velocity illustrates that dish tapping still leads 
to activation of ALM while the worm does no longer show a mobility response (Figure 3.38a).

(a)

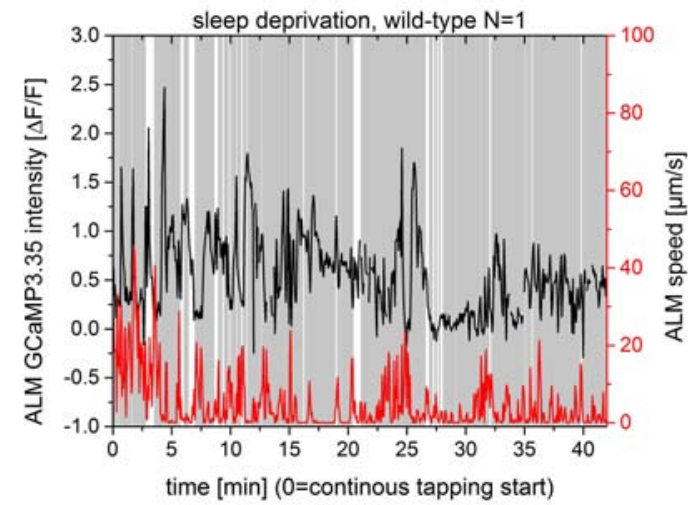

(b)

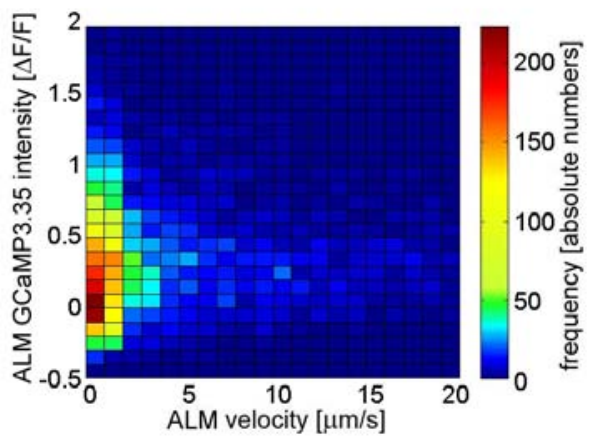

Figure 3.38: The sleep homeostat does not inhibit ALM sensory neuron activity in order to drive quiescence. (a) ALM activity and velocity for a typical single sleep deprived animal. Dish tapping stimulation is indicated by gray shading. (b) ALM activity vs. velocity histogram during active sleep deprivation by dish tapping averaged for $\mathrm{N}=9$ animals.

For quantification of this effect I created a histogram of ALM activity and velocity (Figure 3.38b). From this diagram one can deduce that although the animal is immobile ( $0=$ ALM velocity) ALM GCaMP3.35 activity can be as elevated as 100\% above baseline level. This means that the sleep homeostat does not inhibit ALM sensory neuron activity in order to drive quiescence.

\subsubsection{Homeostatic quiescence induction while sensory neurons still sense deprivation stimulus is mediated by RIS}

To relate the activity pattern of ALM and PLM to RIS I measured them at the same time. For simultaneous imaging of these three neurons the focus was adjusted to center ALM allowing to resolve PLM and RIS due to their similar lateral position within the animal.

For the wake tap control ALM activity persistently stays above basal levels while being stimulated. This is accompanied by sustained mobilization of the animal and low RIS activity (Figure 3.39b). How does RIS and ALM activity relate to each other while sleep depriving the animal? Figure 3.40 shows a ALM-RIS intensity histogram that indicates a linear dependency. Interestingly RIS and ALM activity peaks simultaneously while a sustained tapping stimulus is applied to sleep deprive the animal (Figure 3.39a). For the late part of sleep deprivation both RIS and ALM activity decreases. This implies two things. First, RIS is not inhibiting ALM sensory neuron activity while actively driving 
(a)

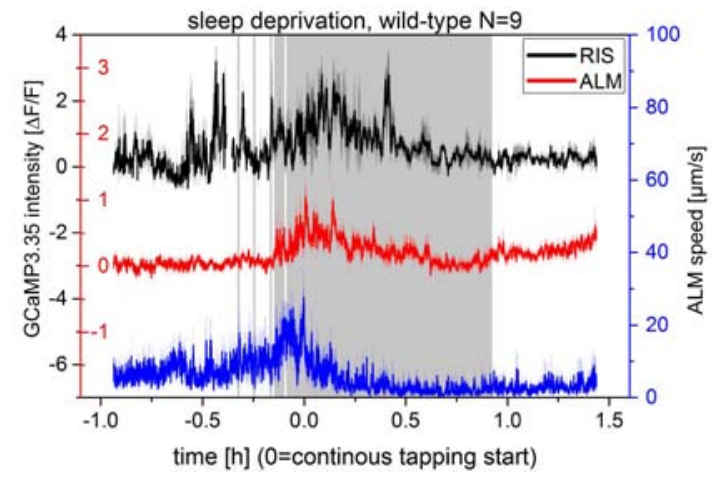

(c)

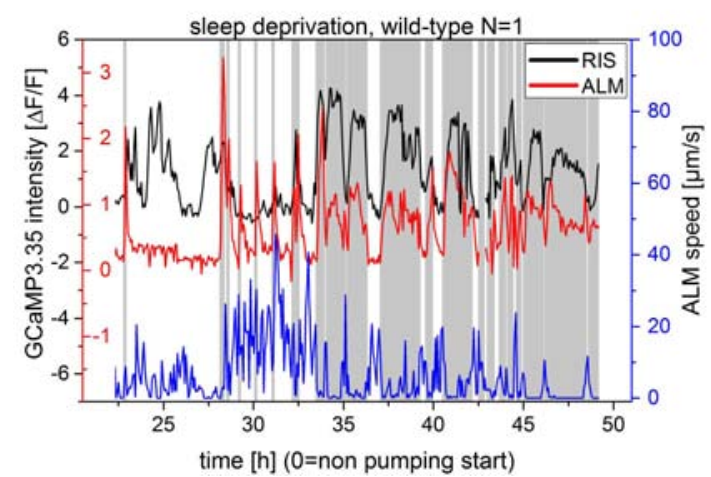

(b)

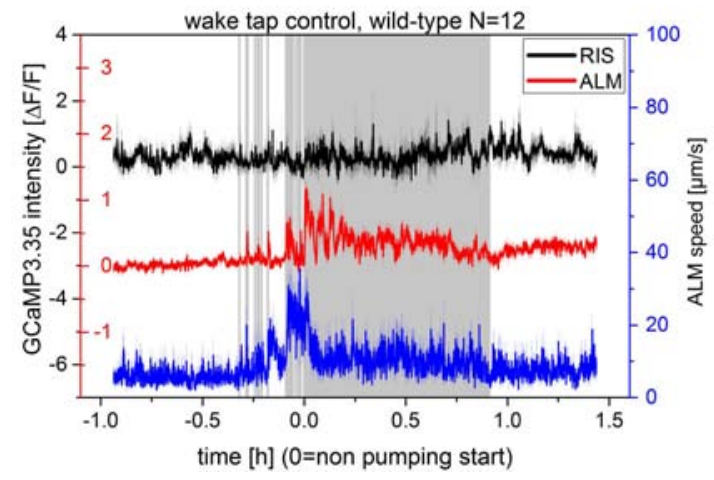

(d)

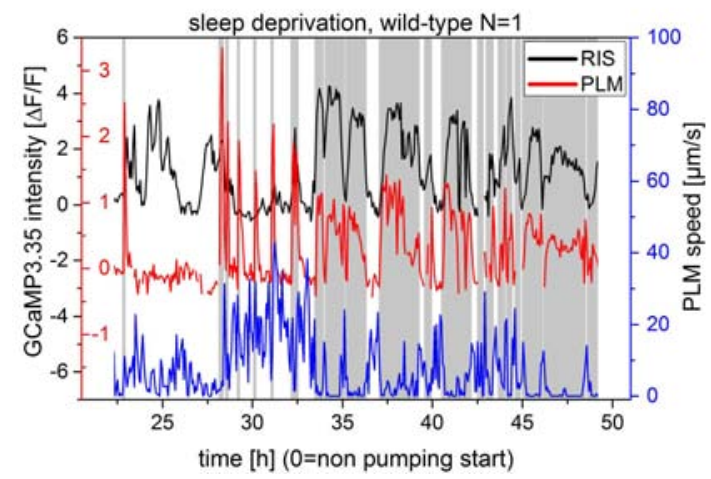

Figure 3.39: Mechanosensory neuron activity is not inhibited while RIS activity is peaking. (a) RIS/ALM activity and ALM speed time course for (a) sleep deprivation averaged for 9 animals and (b) wake tap control averaged for 9 animals. (c) Single animal RIS/ALM activity and ALM speed time course. (d) Single animal RIS/PLM activity and PLM speed time course. Dish tapping stimulation is indicated by gray shading.

immobilization during sleep. Second, for the late part of sleep deprivation the tapping stimulus barely activates ALM and PLM, therefore triggering less RIS activation. To elucidate the relationship between ALM and RIS activity I applied a rigid tapping protocol to the L1-L2 cycle of the worm. The protocol consists of 10 seconds without stimulation, followed by 20 seconds of tapping, and again 10 seconds without stimulation. ALM activity increases continuously during the first 10 seconds of tapping and then finally saturates at an intensity level 100\% higher than basal activity. Ten seconds after the end of tapping ALM activity reaches almost basal levels again (Figure 3.41a). Intriguingly RIS activity is very similar to ALM. Over the first 10 seconds of stimula- 


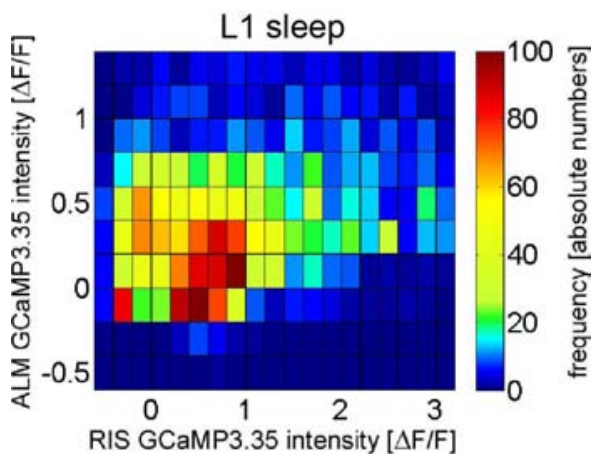

Figure 3.40: ALM activity vs. RIS activity histogram. The color of each square represents the frequency for a given combination of ALM-RIS intensities.

(a)

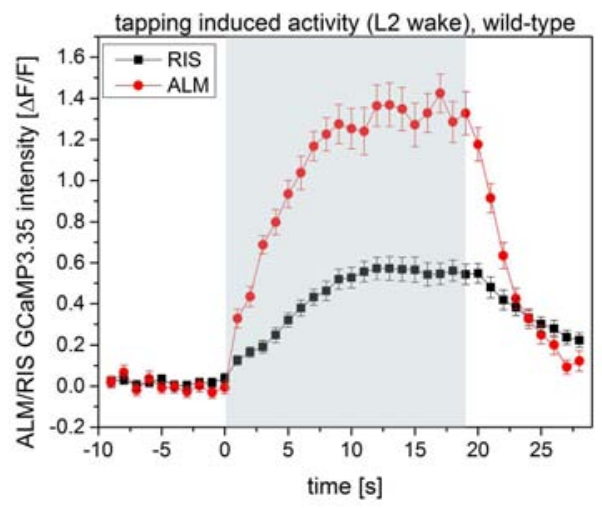

(b)

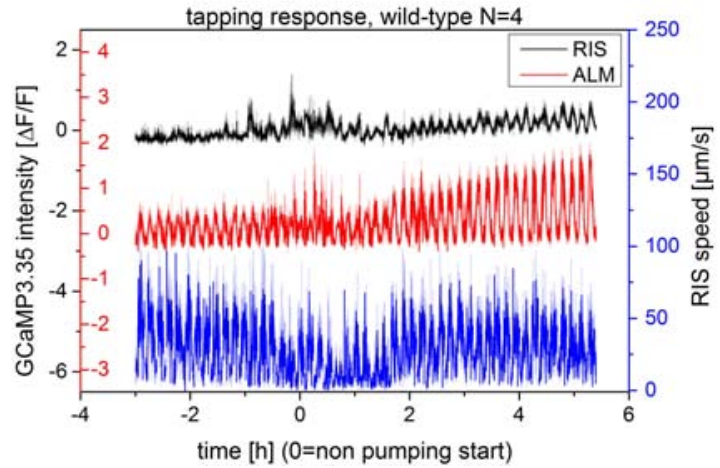

Figure 3.41: RIS dampens tapping induced mobility by activating similar to ALM. Measurements are taken every 10 minutes and consist of 10 seconds without stimulation, followed by 20 seconds of tapping and again 10 seconds without stimulation. (a) Averaged calcium activity of RIS and ALM for 6-8 hours after non pumping start. Dish tapping stimulation is indicated by gray shading. (b) Averaged calcium activity of RIS and ALM and RIS velocity across time.

tion RIS activity rises, then saturates for the next 10 seconds of tapping. After the end of stimulation RIS activity decreases but does not yet reach basal level after 10 seconds. By looking at the time course of ALM and RIS activity in reaction to tapping one notices ALM activity almost perfectly reflecting the stimulus for all time points except for L1 sleep (Figure 3.41b). RIS does not activate upon tapping in L1 wake but does so strongly during sleep and consecutive L2 wake. RIS activity upon tapping is highest during L1 sleep. 


\subsection{Molecular and genetic basis of RIS signaling}

To better understand the molecular basis of RIS I investigate on the transcription factor lim-6, that was known to regulate RIS differentiation. In the following I demonstrate that $\mathrm{lim}-6$ has a sleep phenotype and show the effects of lim- 6 on gene expression as revealed by transcriptome analysis. Of the most interesting genes I analyzed $\mathrm{KO}$ mutants. Additionally I investigated the role of neurotransmitter and neuropeptide signaling pathways on the sleep homeostat. Finally I examined several transmembrane channel and receptor mutants, that either have a sleep and/or arousal phenotype.

Effects of irbesartan on gene expression revealed by transcriptome analysis

\subsubsection{The LIM homeobox transcription factor lim-6}

lim-6 is a transcription factor that belongs to the LIM homeobox transcription factor family. It has homologs in Drosophila, mouse and human (Lmx1b). Phylogenetically closest to lim-6b in C. elegans are the orthologs Lmx1b in mouse, CG4328 and CG32105 in Drosophila (Figure 3.42).

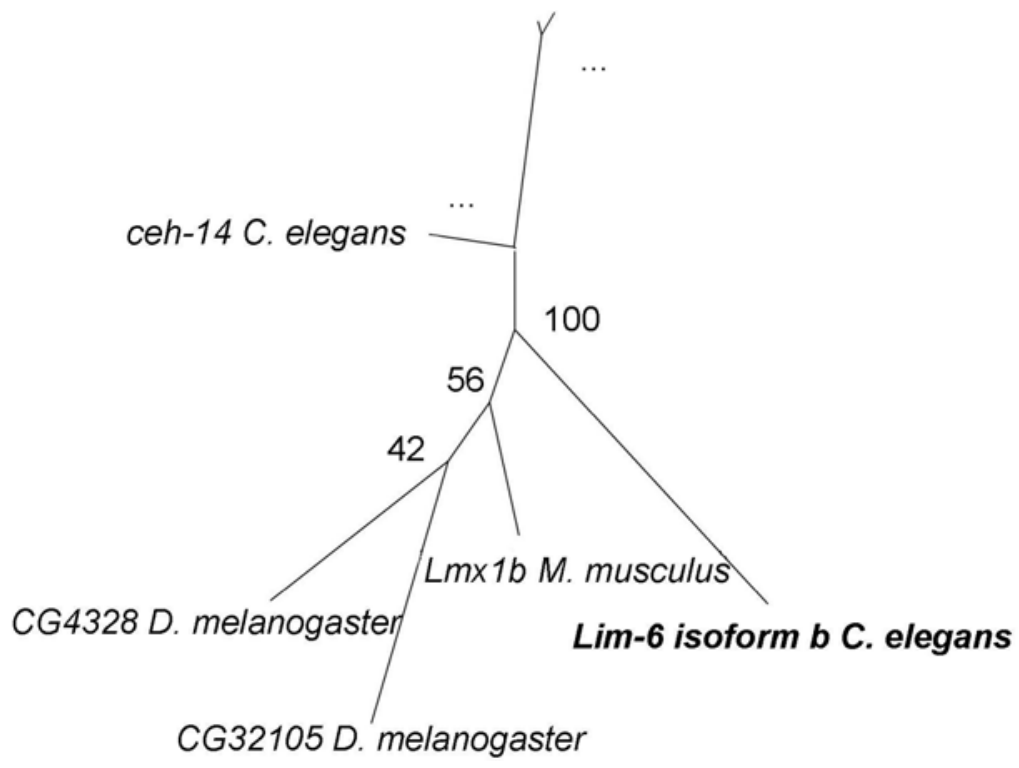

Figure 3.42: Reduced scheme of the phylogenetic tree of lim-6b. C. elegans has been compared with M. musculus and D. melanogaster. Homology has been calculated using the program MEGA (molecular evolutionary genetics analysis), www. megasoftware. net

The mouse $\mathrm{KO}$ mutant of $L m x 1 b$ (flox/flox;ePet-Cre/+) in serotonergic neurons shows an increase in wakefulness, an impaired hypercapnia-induced arousal and frequent and severe apnea [87]. In C. elegans lim-6 has not yet been related to sleep. However it is known that lim- 6 together with ceh-14 regulates gene expression of genes with temporally regulated profiles of expression, defining the competence of the neuron PVT to 
respond to a temporal cue [88]. The regulation of sleep and waking could be based on a similar mechanism, with lim-6 defining the competence of the neuron RIS to respond to temporal cues of sleep and waking. In C. elegans lim- 6 is expressed in 9 neurons: RIS and RIGL/R (interneurons); AVL, PVT, DVB, RMER/L (motor neurons) and ASEL (sensory neuron). lim-6 is expressed from the embryonic stage throughout the larval stages.

It has been reported that 1 im- 6 regulates RIS differentiation [89]. Therefore I investigated on a KO mutant for the lim-6 gene, lim-6(nr2073).

\subsubsection{Sleep phenotype of lim-6 mutants}

Wild-type animals exhibit bouts of immobility for about $50 \%$ of sleep time while the average mobility decreases by $50-80 \%$. lim-6(nr2073) mutants rarely immobilize during sleep and average mobilities are reduced by only $30 \%$ (Figure 3.43 ).

To confirm that the lim- 6 gene is causing the sleep phenotype I investigated a second lim-6 KO mutant. The only other existing lim-6 KO mutant is the strain lim-6(tm4836) from the National Bioresource Project (NBP) (http: / / www . shigen.nig.ac.jp/c. elegans). The NBP strains are created by heavy mutagenesis and contain several $\mathrm{KO}$ mutations. To remove most mutations except the one in lim-6, I backcrossed this strain six times with wild-type. Phenotypic analysis showed that the lim-6(tm4836) KO mutant has an even stronger light-sleep phenotype than lim-6(nr2073) (Figure 3.44). Total immobility is nearly absent, the average mobility during sleep decreases only by $8 \%$ compared to L1 wake. 
(a)

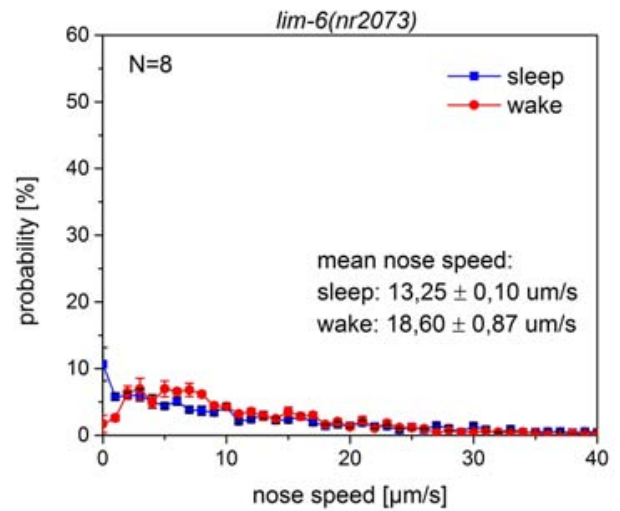

(c)

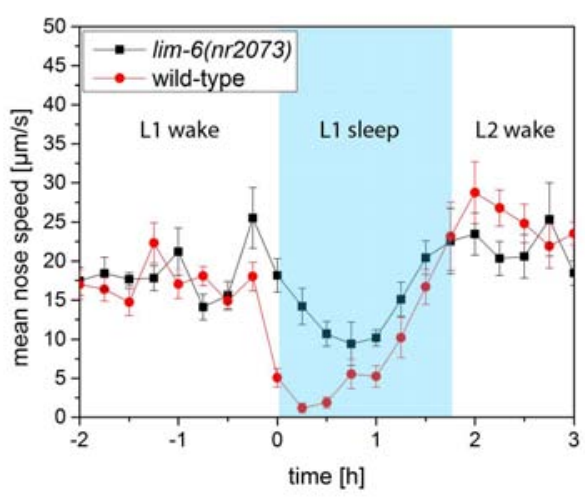

(b)

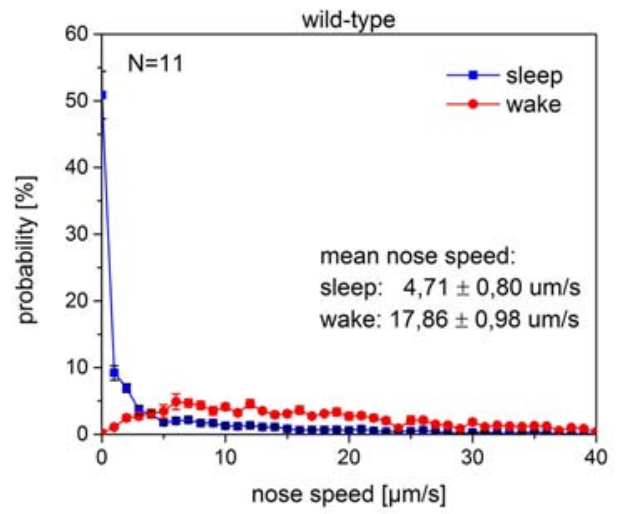

(d)

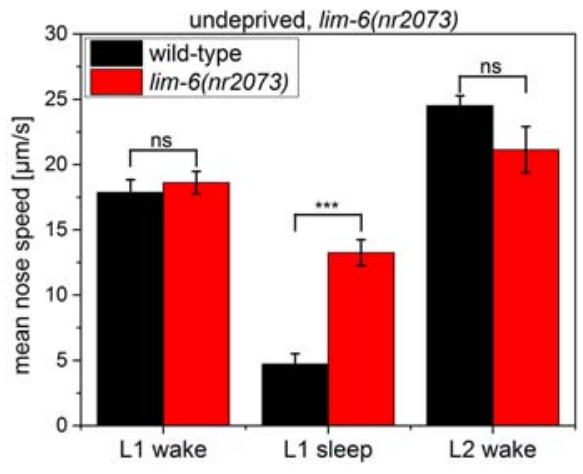

Figure 3.43: lim-6(nr2073) KO mutant exhibits a light-sleep phenotype. Probability distribution of nose speeds during L1 wake and L1 sleep in (a) lim-6(nr2073) and (b) wild-type. (c) Nose speed time course for lim-6(nr2073) and wildtype control. (d) Mean nose speed for lim-6(nr2073) and wild-type in late L1 wake, L1 sleep and early L2 wake. ${ }^{* * *}$ denotes statistical significance with $\mathrm{p}<0.001$, Welch test. 
(a)

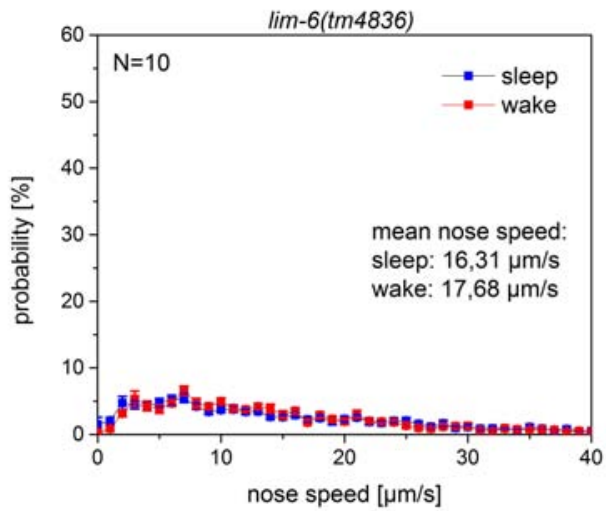

(c)

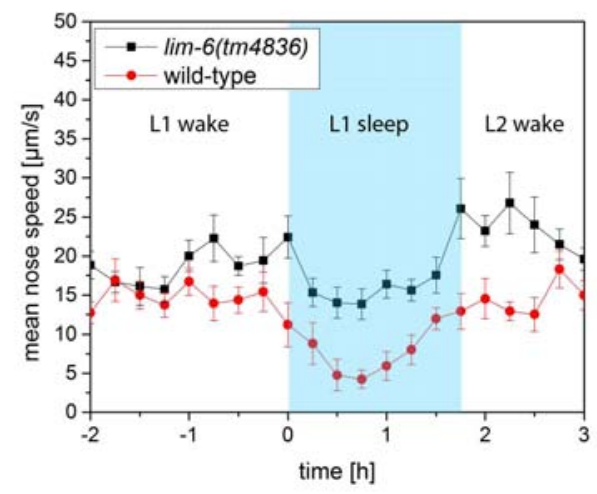

(b)

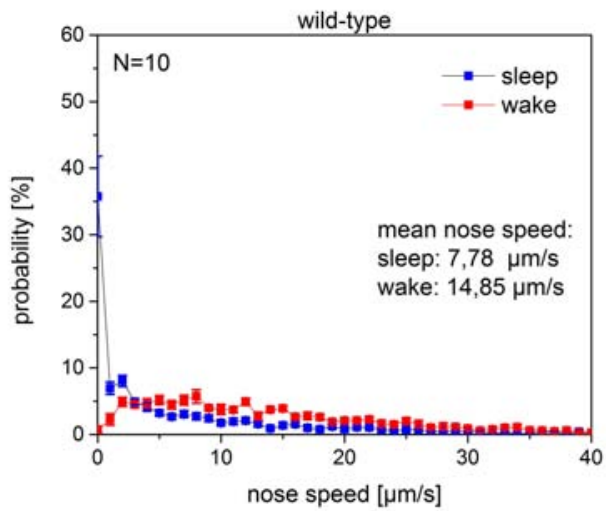

(d)

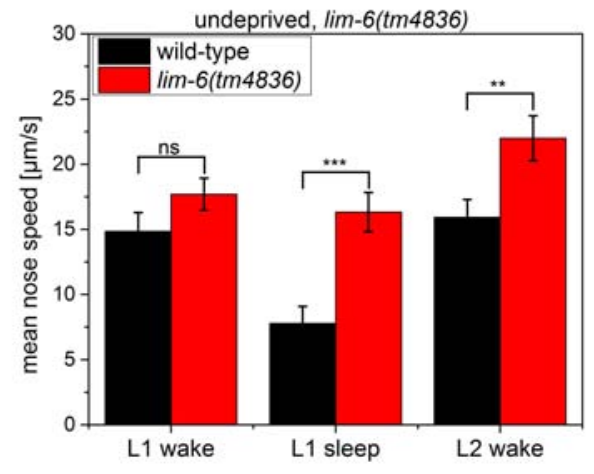

Figure 3.44: lim-6 KO mutant lim-6(tm4836) exhibits a light-sleep phenotype. Probability distribution of nose speeds during L1 wake and L1 sleep in (a) lim6(tm4836) and (b) wild-type. (c) Nose speed time course for lim-6(tm4836) and wild-type control. (d) Mean nose speed for lim-6(tm4836) and wildtype control in late L1 wake, L1 sleep and early L2 wake. ${ }^{* *}$ denotes statistical significance with $\mathrm{p}<0.01,{ }^{* * *}$ denotes statistical significance with $\mathrm{p}<$ 0.001, Welch test. 


\subsubsection{Rescue of the lim-6 mutant sleep phenotype}

To rescue the lim-6 sleep phenotype I decided to use an existing rescue line otIs157[lim$6 r:: G F P+p R F 4($ rol-6(su1006)) $]$ that represents a lim- 6 rescue containing the lim- 6 coding and non-coding regions tagged with a GFP and the dominant rol-6(su1006) mutation. In older larvae and adult animals the dominant rol-6(su1006) mutation leads to persistent rolling in circles instead of normal locomotion. However young larvae superficially exhibit normal locomotion.

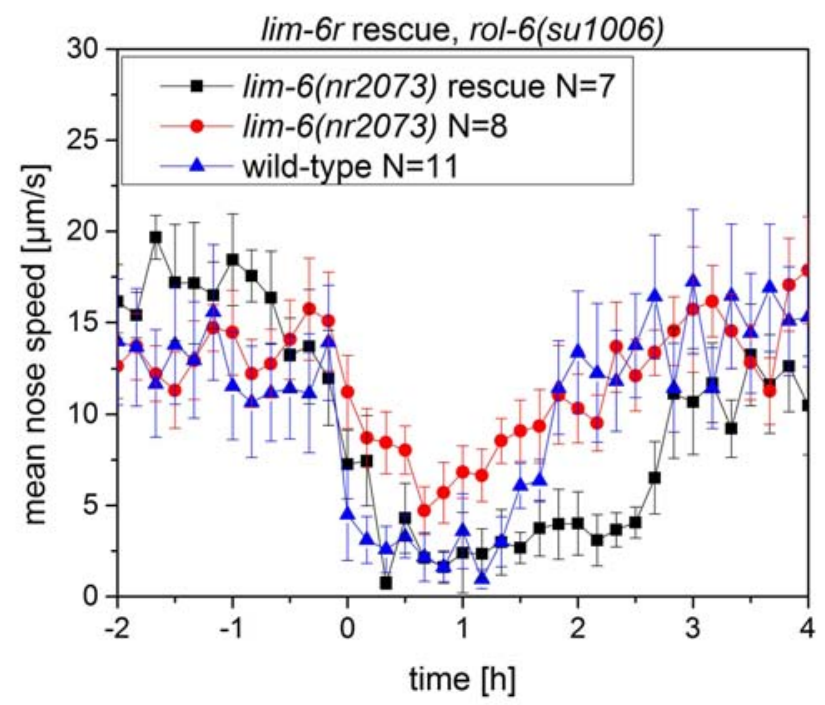

Figure 3.45: lim-6(nr2073) Rescue with lim-6-r tagged with GFP and the rol-6(su1006) mutation

The lim-6r strain rescues the lim-6(nr2073) sleep phenotype (Figure 3.45). Rescued lim-6(nr2073) spend a large portion of their non pumping period immobile and average velocities during sleep are similar to wild-type. For the lim-6r,lim-6(nr2073) mutants the time between the restart of pumping and the complete shedding of the old cuticle takes about one hour longer than for wild-type and lim-6(nr2073) control. To exclude that the additional rol-6(su1006) mutation influences the sleep phenotype I decided to make rescue constructs carrying only a lim-6ab rescue tagged with the fluorescent protein mKate2. My hypothesis is that lim-6 is required only in RIS to rescue the wild-type sleep phenotype. A RIS cell specific lim- 6 rescue requires a promoter expressing solely in RIS. Single cell promoters only exist for a small fraction of the three-hundred and two neurons. RIS being the exception with the neurexin like receptor gene $n l r-1$ expressing in RIS and the pharynx only (personal communication Marie Gendrel). I crossed the lim-6(tm4836) KO mutant into a transgenic strain that expresses GFP under the $n l r-1$ promoter, that was kindly provided by Marie Gendrel. However expression of $n l r-1$ is strongly downregulated in the lim- $6 \mathrm{KO}$ mutant. Furthermore expression of $n l r-1$ does 


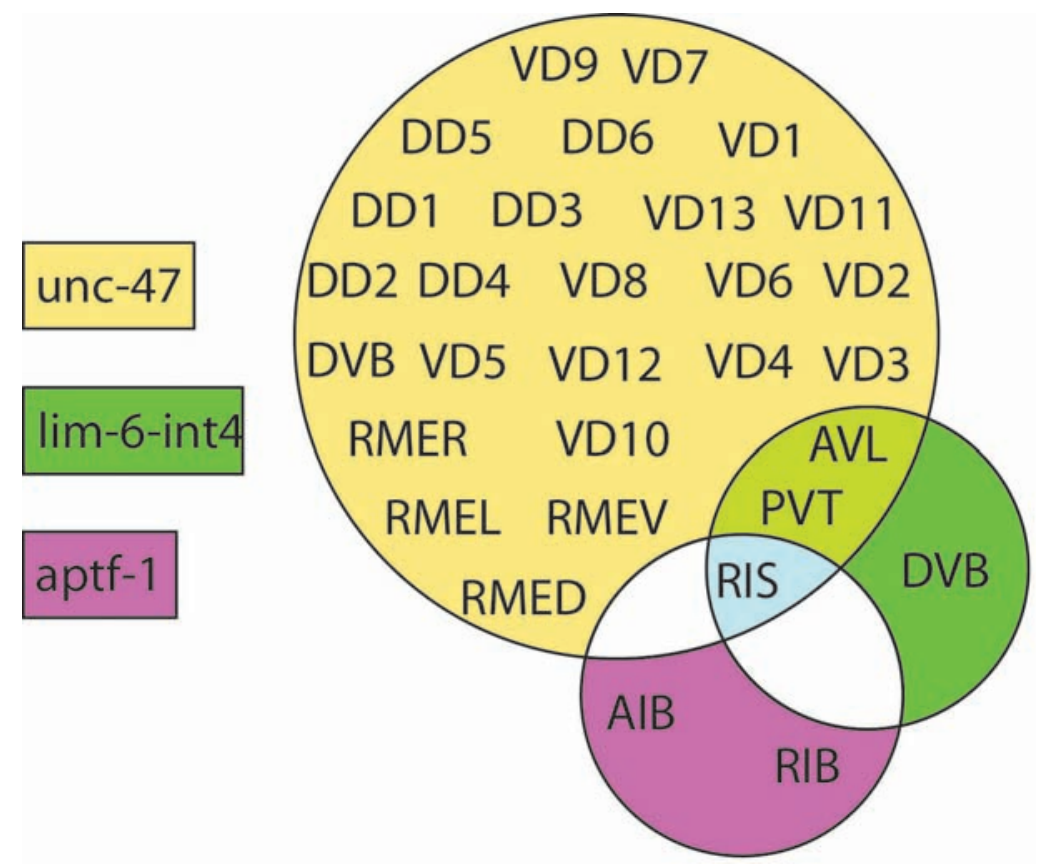

Figure 3.46: Neural expression of the three promoters unc-47-nu486, aptf-1 and lim-6int4. aptf-1 expression overlaps with unc-47-nu486/lim-6-int4 only in RIS.

not seem to be restricted to RIS only. Another possibility to test the role of RIS would be a rescue for two promoters with their expression only overlapping in RIS. This is true for the combination of the aptf-1 and unc-47-nu486 promoter or of aptf-1 and the lim-6-int4 promoter (Figure 3.46). A rescue for one of these two combinations would therefore let me allow to conclude that the lim- 6 knockout in RIS is really at the base of the sleep phenotype. lim-6-int4 corresponds to the fourth intron of the lim-6 gene, that previously has been falsely annotated as lim-6-int3 [89]. For all three promotors I created constructs carrying the lim- $6 a b$ rescue and mKate 2 that is used as an expression control. I injected all three constructs and obtained for unc-47-nu486 and lim-6-int4 several extra-chromosomal lines. Expression for the $u n c-47-n u 486$ promoter was as reported, but additionally to the reported neurons the lim-6-int4 promoter expressed in some other tissue. Both extra-chromosomal arrays with expressing lim-6ab rescue constructs under the lim-6-int4 promoter did not rescue the sleep phenotype (Figure 3.47a, $3.47 \mathrm{~b})$. For the $u n c-47-n u 486:: \mathrm{lim}-6 a b$ there was no rescue of the sleep phenotype for most animals either (Figure 3.47c). A partial rescue for some individual animals occurred, but could not be related to a difference in the expression pattern of the rescue construct.

To pinpoint the reasons for the failure of the lim- $6 a b$ constructs to rescue the lim- 6 sleep phenotype, I double checked the annotation of the lim- 6 gene. The official annotation of the lim- 6 gene consists of two splice variants $l i m-6 a$ and lim- $6 b$. RNA Sequencing of wild-type and lim-6(nr2073) mutants (see section 3.6.6) only detected transcripts of the 
(a)

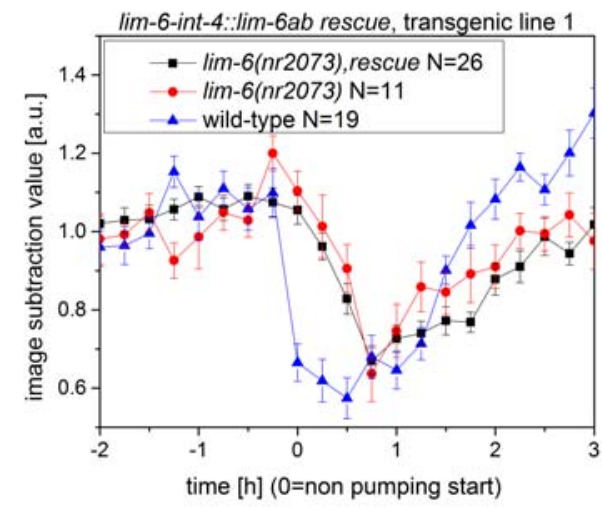

(c)

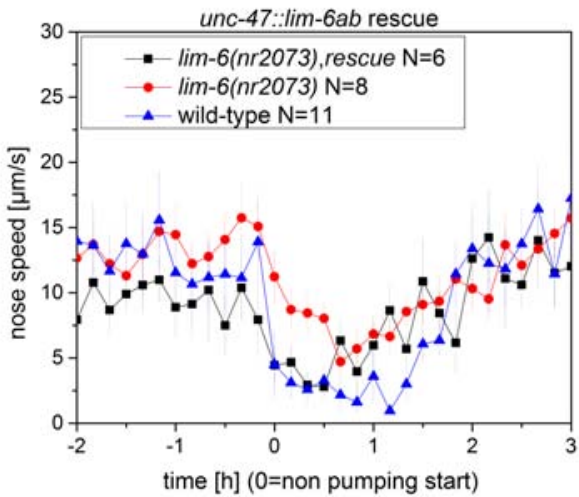

(b)

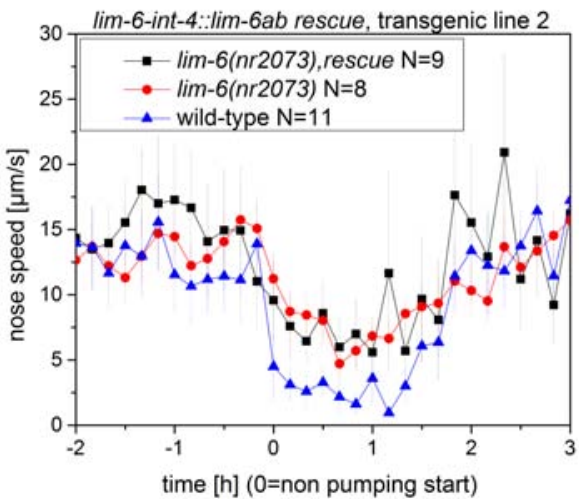

Figure 3.47: lim-6ab rescue constructs do not rescue the lim-6 sleep phenotype. $(\mathrm{a}, \mathrm{b})$ lim-6ab expressed under the lim-6-int4 promoter does not rescue the lim6(nr2073) sleep phenotype. (c) lim-6ab expressed under the unc-47-nu486 promoter partly rescues the sleep phenotype.

splice variant $l i m-6 b$. Therefore I generated new rescue constructs for the aptf-1, unc-47$n u 486$ and lim-6-int4 promoter carrying only the splice variant lim-6b and a mKate 2 that is used as an expression control. For each of these constructs I did two transformations using microparticle bombardment and obtained three integrated lines for the lim-6-int4 promoter, one extrachromosomal for the unc-47-nu486 promoter, but none for the apft-1 promoter. I proceeded only with the integrated lim-6-int 4 strains and crossed them into the lim-6(tm4836) mutant. I measured the sleep wake cycle of the lim-6-int4::Iim-6b rescue animals together with wild-type and lim-6(tm4836) animals. However, behavioral analysis of the sleep wake cycle did not show a rescue of the lim-6 sleep phenotype (Figure 3.48). 


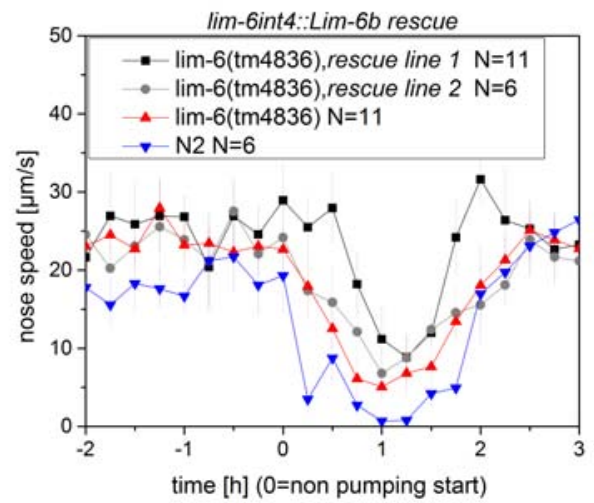

Figure 3.48: The lim-6b rescue construct expressed under the lim-6-int4 promoter does not rescue the lim-6 sleep phenotype.

\subsubsection{RIS activity of undeprived lim- 6 mutants}

In wild-type, lim-6 expression in RIS is slightly reduced during L1 sleep (Figure 3.49).

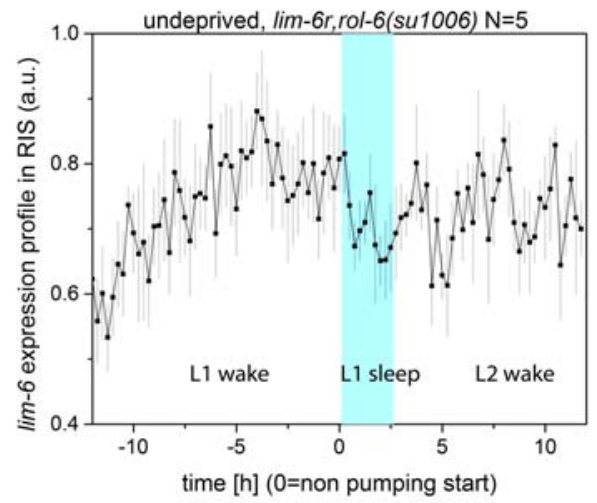

Figure 3.49: lim-6 expression profile in RIS from early L1 to mid L2. Expression levels were obtained by measuring fluorescent signal from LIM-6 protein tagged with GFP.

To observe RIS activity in the lim-6(nr2073) $\mathrm{KO}$ mutant I crossed it into a transgenic line expressing GCaMP3.35 under the aptf-1 promoter. From the literature it is known that the transcription factor lim-6 is needed for the expression of some genes in RIS, e.g. dop-1 and ser-4 [90]. Unfortunately aptf-1 expression is strongly downregulated in the lim-6(nr2073) KO mutant compared to wild-type (Figure 3.54). Although the GCaMP3.35 signal is very faint it might be still sufficient to be imaged using a confocal microscope and a 40x oil objective. The combination of a confocal microscope and a high magnification only allows tracking neural activity in a small vertical layer. Therefore I took a z-stack at each time point and later manually selected the correct focal 
plane. I recorded GCaMP3.35 and mKate2 simultaneously using a beam splitter. The mKate2 signal is used as an expression control. RIS position can be tracked easily using the brighter mKate2 signal that is co-localizing with the fainter GCaMP3.35 signal. To correct for variation in intensity due to differences in the focal plane I calculated the ratio between the GCaMP3.35 and the mKate2 signal (Figure 3.50). Despite the bad signal to noise ratio RIS activity seems to peak around the sleep onset and to drop in consecutive sleep. To validate this finding I needed a promoter with better RIS GCaMP expression in the lim-6 $\mathrm{KO}$ mutant.

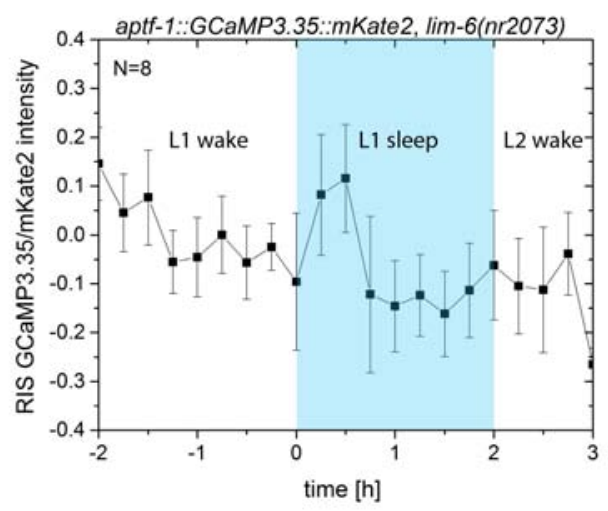

Figure 3.50: RIS activity in the lim-6(nr2073) mutant using the aptf-1 promoter to drive GCaMP3.35 and mKate2 expression. Shown is the ratio of GCaMP3.35 over mKate2 activity, which were recorded simultaneously using a beam splitter.

I created a construct carrying GCaMP3.35 plus a mKate2 under the lim-6-int4 promoter. Integration of the construct did not work and resulting extrachromosomal lines were so badly transmitting and expressing in a mosaic way that measurements were not possible. Another good candidate to drive expression in RIS is the $n l r-1$ promoter. However, similar to aptf-1, expression of $n l r-1$ is strongly downregulated in the lim- $6 \mathrm{KO}$ mutant. Tsalik et al. reported that unc-47 expression is barely downregulated in the lim- $6 \mathrm{KO}$ mutant [90]. I crossed a transgenic line expressing GCaMP3 under the unc-47 promoter into the lim-6(tm4836) mutant to measure its RIS activity. RIS activity was measured using a confocal microscope and a $40 \mathrm{x}$ oil objective by taken z-stacks of GCaMP3 activity every 15 minutes. The sleep onset peak of RIS activity seems to be lower but more widespread than for wild-type (Figure 3.51a, 3.51b). However by aligning the animals by the RIS sleep onset peak value, instead of non pumping start, one obtains a similar RIS sleep onset peak as wild-type (Figure 3.51c, 3.51d). lim-6(tm4836) RIS activity is not significantly changed compared to wild-type control (Figure 3.51e). Only the temporal relation between RIS sleep onset peak and the start of the non pumping phase is impaired. 
(a)

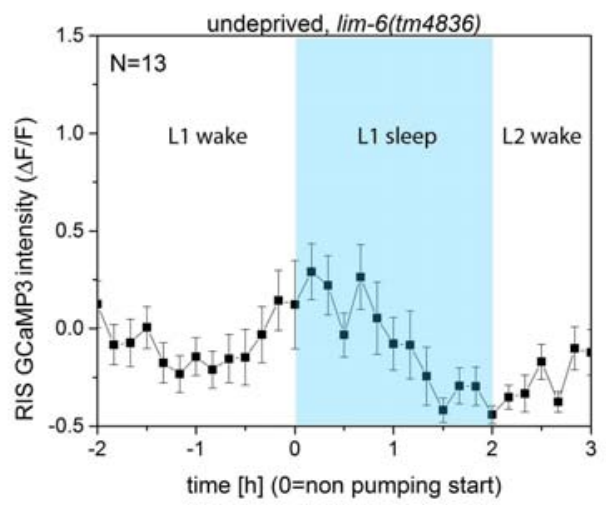

(c)

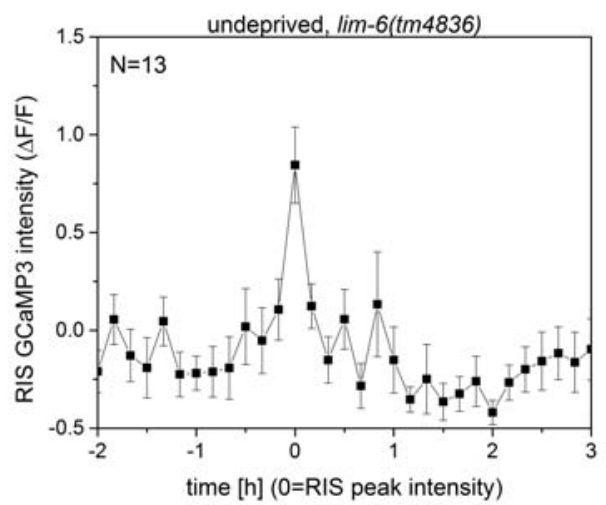

(b)

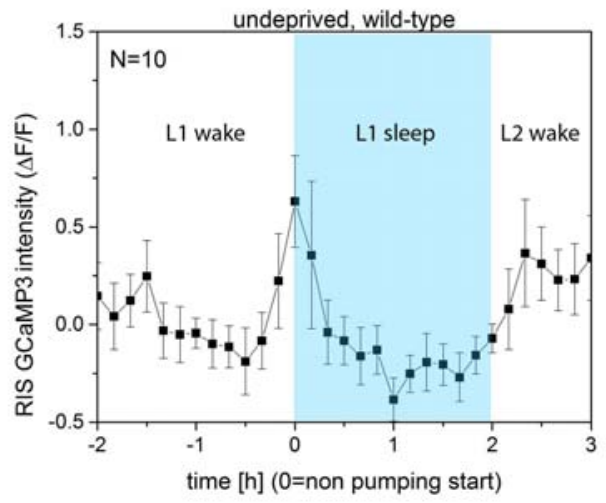

(d)

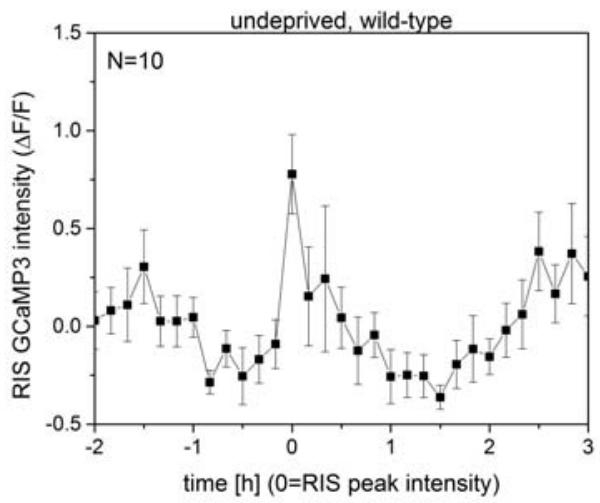


(e)

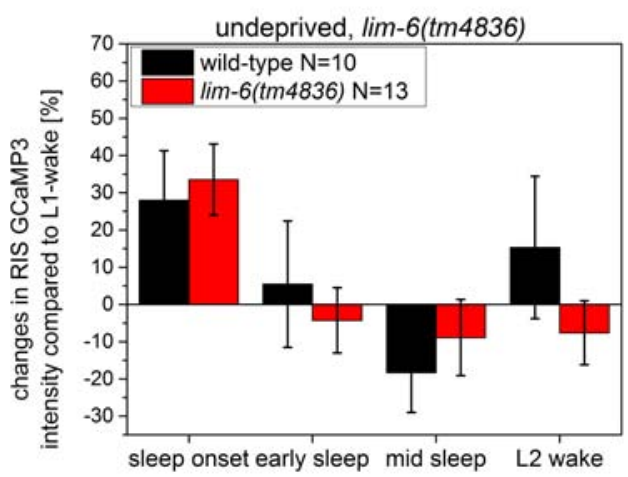

Figure 3.51: Undeprived lim-6(tm4836) have the wild-type sleep onset peak for RIS activity. The $u n c-47$ promoter was used to drive GCaMP3 expression in RIS. (a, c) lim-6(tm4836) RIS activity aligned by (a) non pumping start and (c) RIS sleep onset peak intensity. (b, d) Wild-type RIS activity aligned by (b) non pumping start and (d) RIS sleep onset peak intensity. (e) Comparison of lim-6(tm4836) (red) with wild-type control (black) that were aligned by RIS onset peak. Average RIS values were calculated for a time window of 20 minutes. The differences in RIS activity between lim-6(tm4836) and wild-type were not significant. Statistical significance was assessed using the Welch test. 


\subsubsection{Sleep deprivation and its effect on RIS activity in lim-6 mutants}

To investigate the effect of sleep deprivation upon RIS activity in the lim- $6 \mathrm{KO}$ mutant presented the problem that simultaneous dish tapping and z-stacks measurements are not possible. Therefore I had to record from a single z-layer and to manually discard all images where RIS was outside the focal plane. I overlayed and averaged the resulting patches of RIS activity and found that $\lim -6(\mathrm{tm} 4836)$ mutants show an increase of RIS activity upon sleep deprivation (Figure 3.52). Due to the lack of an appropriate control I cannot compare this to wild-type. The sleep detection algorithm detected sleep correctly in seven out of eight cases for the lim- $6 \mathrm{KO}$ mutants.

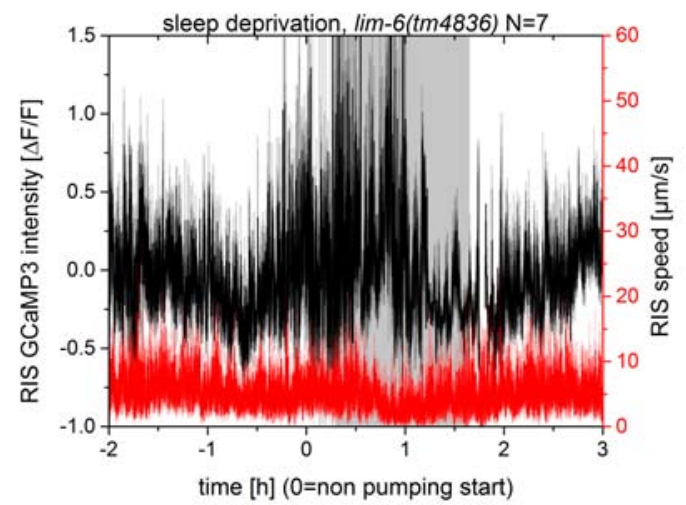

Figure 3.52: lim-6(tm4836) shows an increase of RIS activity upon sleep deprivation. The $u n c-47$ promoter was used to drive GCaMP3 expression in RIS. Shown are the overlayed and averaged RIS activity patches of $\mathrm{N}=7$ sleep deprived lim-6(tm4836) mutant animals. Dish tapping stimulation is indicated by gray shading.

\subsubsection{RNA Sequencing of lim-6 mutants}

As a transcription factor lim- 6 acts by altering gene expression of possibly thousands of genes. To find out which genes are regulated by lim-6 I did RNA Sequencing. I collected lim-6(nr2073) mutants and wild-type eggs manually and transferred them into Trizol solution, thus killing them immediately. Simultaneously with my RNA Sequencing of lim-6(nr2073), Michal Turek did RNA Sequencing of aptf-1(gk794) mutants. RNA Sequencing of the samples was done by the Transcriptome Analysis Laboratory Goettingen (TAL). The analysis revealed 122 genes that were upregulated by lim- 6 and 22 genes that were downregulated by lim-6 (Figure 3.53).

In the top ten of genes that are downregulated in lim-6(nr2073) mutant animals is as expected lim-6 itself, implying the reliability of the assay (Table 3.2). An interesting hit is the nuclear hormone receptor $n h r-198$, as nuclear hormone receptors are known to regulate developmental cues [91]. 


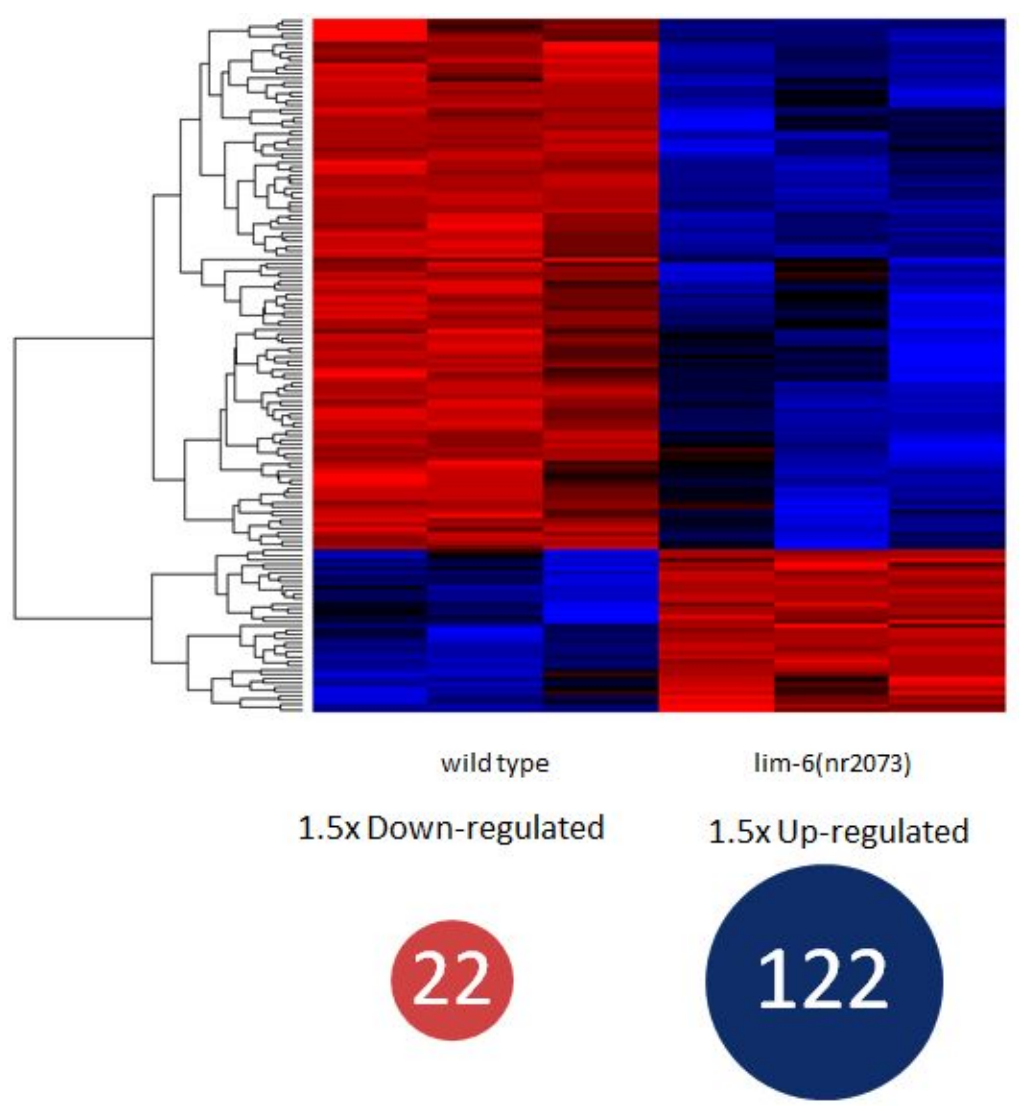

Figure 3.53: lim-6(nr2073) and wild-type RNA Sequencing. Shown in red/blue are the genes that are downregulated/upregulated in wild-type (leftside) and lim6(nr2073) (right side).

\begin{tabular}{|l|l|}
\hline \multicolumn{2}{|c|}{ Top 10 downregulated } \\
\hline Gene Name & function \\
\hline Y68A4B.3 & NA \\
F36D1.23 & spectrin beta chain \\
LIM-6 & lim-6 isoform b transcription factor \\
F30A10.4 & Branching Enzyme \\
K05C4.9 & Leucine rich repeats \\
F55G11.7 & CUB-like domain \\
C49A1.5 & Glycosyltransferase family \\
NHR-198 & Nuclear Hormone Receptor \\
H05L03.3 & NA \\
CDR-7 & Cadmium-inducible lysosomal protein \\
\hline
\end{tabular}

Table 3.2: Top ten of genes that are downregulated in lim-6(nr2073) mutant animals. 


\subsubsection{Link between aptf-1, lim-6 and RIS}

The two transcription factors aptf- 1 and $\lim -6$ are both essential for normal sleep. To understand better how they act, it is important to know if they are part of the same sleep regulation cascade, or if they act independently of each other. The RNA Sequencing results of aptf-1 and lim- 6 did not indicate any mutual regulation. To detect subtle changes of expression levels or pattern I investigated whether expression levels of a aptf-1 reporter construct are changed in the lim-6(nr2073) compared to wild-type. aptf1 expression in lim-6(nr2073) is similar to wild-type with the exception of RIS (Figure 3.54). aptf-1 RIS expression is downregulated about $75 \%$. Therefore one possibility

(a)

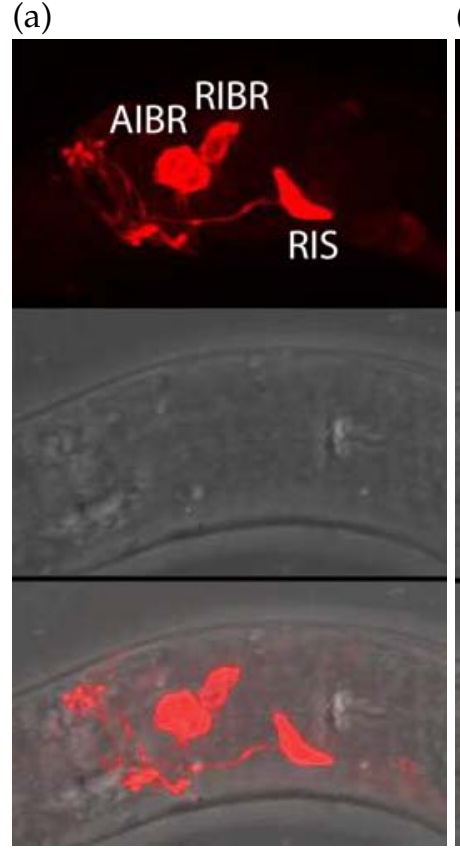

(b)

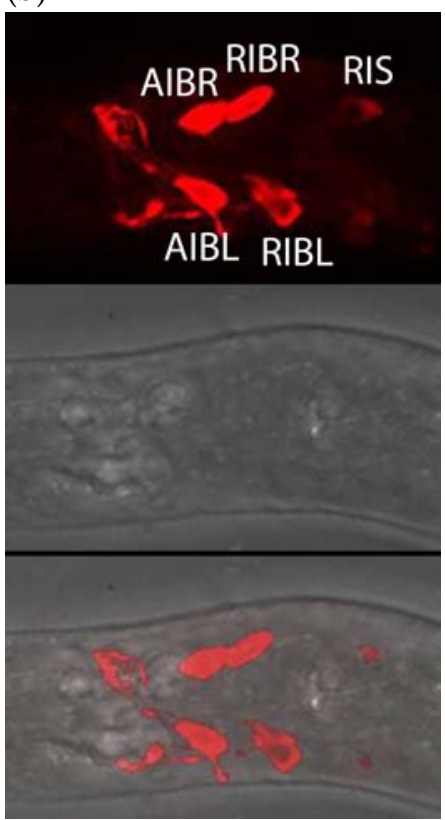

Figure 3.54: Regulation of aptf-1 expression in RIS by lim-6. (a) Wild-type aptf-1 expression and (b) lim-6(nr2073) aptf-1 expression. Expression of aptf-1 looks similar to wild-type in lim-6(nr2073), except for the strong downregulation of expression in RIS.

is that the sleep phenotype of $l i m-6 \mathrm{KO}$ mutant is due to a downregulation of aptf-1 gene expression in RIS. Activation of aptf-1-expressing cells with ChR2 leads to immobilization in wild-type [40]. lim-6(nr2073) mutants with ChR2 expressed under the aptf-1 promoter do not immobilize in response to blue light, but slightly increase their mobility (Figure 3.55). This is probably due to too low expression levels of ChR2 in RIS. To investigate whether ChR2 expression in RIS is able to drive immobilization in the lim- $6 \mathrm{KO}$ mutant I needed another promoter. I created a ChR2 construct with the lim-6-int4 promoter expressing in RIS and only three other neurons. However, neither microparticle bombardment nor injection resulted in an integrated or a good transmitting extrachromosomal transgenic line. Therefore it was not possible to test whether 


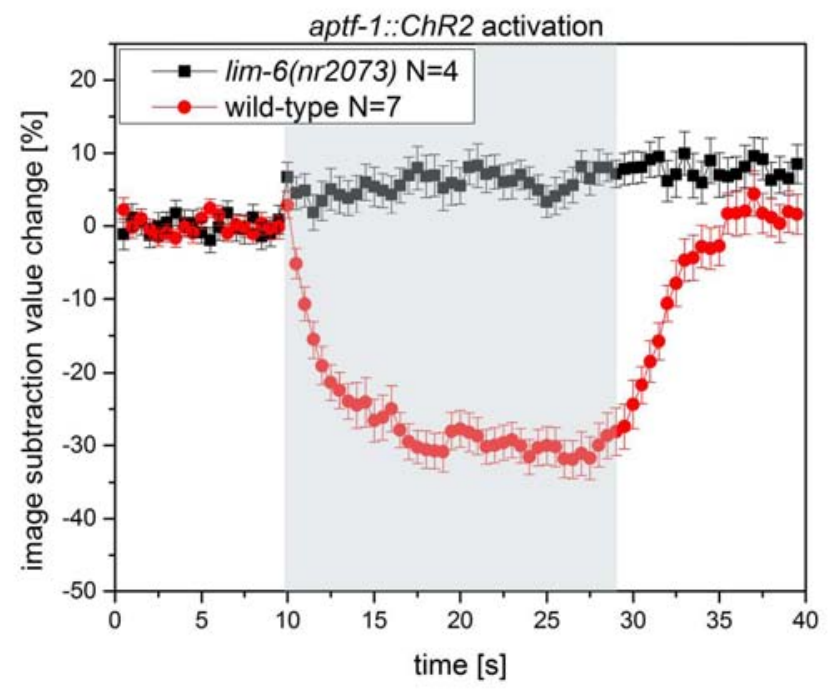

Figure 3.55: Activation of aptf-1-expressing cells with ChR2 does not lead to immobilization in lim-6(nr2073) mutants, but in wild-type animals. lim-6(nr2073) and wild-type have been measured simultaneously. Blue light stimulation is indicated by gray shading.

activation of RIS induces quiescence in the lim-6 mutant.

\subsection{8 aptf-1 RIS phenotype}

aptf-1 KO mutant RIS activity of undeprived animals does not differ much from wildtype [40]. As a baseline for sleep deprivation experiments I measured RIS activity continuously with a temporal resolution of four seconds. As reported previously [40] undeprived aptf-1 (gk794) show the RIS sleep onset activation peak, a consecutive reduction of RIS activity during sleep, and a rise of RIS activity at the end of sleep and beginning of L2 wake (Figure 3.56a). During early and mid sleep and during L2 wake aptf-1(gk794) RIS activity is increased compared to wild-type, while it is lowered at sleep onset (Figure3.56b. Dish tapping during sleep leads to mobilization of the worm and with a short delay to an increase in RIS activity (Figure 3.56c). Instead of automated sleep deprivation I used a predefined tapping protocol that I started manually. Interestingly the correlation between RIS activation and a reduction of velocity can also be observed in the aptf-1(gk794), although RIS activation in reaction to dish tapping is reduced compared to wild-type. This shows that the sleep homeostat is still partly functional in aptf-1 KO mutants. aptf-1, lim- 6 double $\mathrm{KO}$ mutant animals have a similar sleep phenotype as aptf-1 mutant animals (data not shown). 
(a)

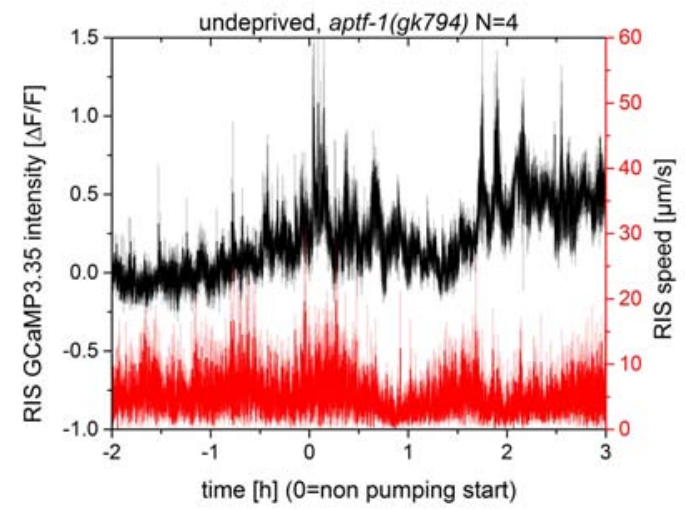

(c)

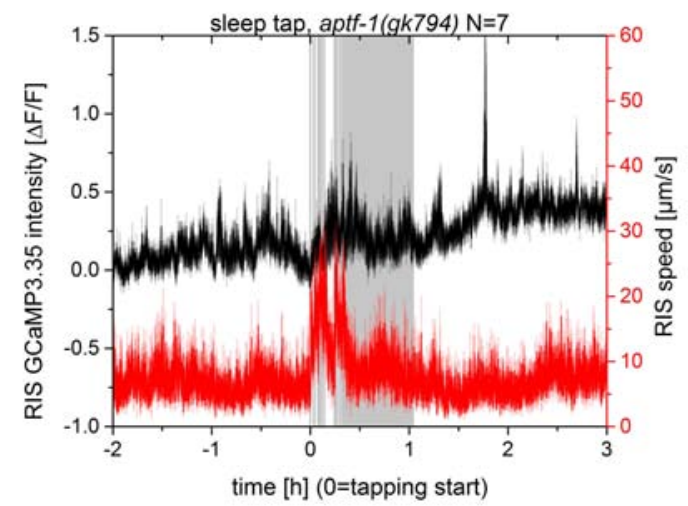

(b)

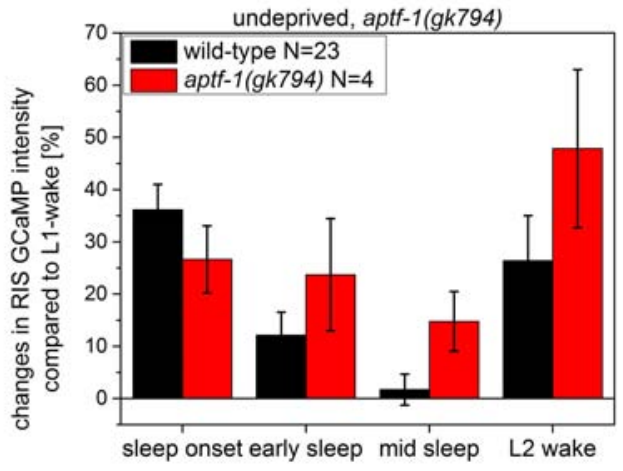

Figure 3.56: aptf-1(gk794) mutant animals exhibit a slightly increased RIS activity compared to wild-type and there is preliminary evidence, that the increase in RIS activity upon sleep deprivation is reduced. (a) Undeprived aptf1 (gk794) RIS activity and velocity averaged for $\mathrm{N}=4$ animals. (b) During early and mid sleep and during L2 wake aptf-1(gk794) RIS activity is increased compared to wild-type and while it is lowered at sleep onset. (c) Averaged RIS activity and velocity for dish tapping during sleep for $\mathrm{N}=7$ animals. Dish tapping stimulation is indicated by gray shading. The same predefined tapping protocol was applied to all animals, which I call sleep tap. An increase in RIS activity is observed in response to prolonged tapping, but appears to be smaller compared to wild-type. 


\subsubsection{Nuclear hormone receptors}

The RNA Sequencing of the lim-6(nr2073) KO mutant gave some interesting candidates that might be responsible for the sleep phenotype. For example $n h r-198$, a nuclear hormone receptor. Nuclear hormone receptors are a class of ligand activated proteins that by regulating the expression of genes influence development and metabolism of an organism [91]. A nuclear hormone receptor would therefore be a good candidate for a mechanism controlling the animal's sleep. RNA Sequencing of the light-sleep mutant aptf-1 vs. wild-type, which has been done simultaneously with my RNA Sequencing, revealed that some other nuclear hormone receptors (nhr-185, nhr-85, nhr-183, nhr-23) are downregulated in aptf-1 mutants. Another nuclear hormone receptor, $n h r-67$, was reported to be expressed in RIS [92]. To find out if any of the above mentioned nuclear hormone receptors regulates sleep I analyzed the sleeping behavior of $\mathrm{KO}$ mutants. However, none of these receptor mutants $\mathrm{KO}$ animals showed a sleep phenotype (Figure 3.57). Table 3.3 summarizes which nuclear hormone receptors I investigated, how they are differently regulated in lim-6 or aptf-1 KO mutants compared to wild-type, and if they show a sleep phenotype. As none of the nuclear hormone receptors investigated exhibited a clear sleep phenotype I did not pursue to investigate more nuclear hormone receptors.

\begin{tabular}{|l|l|l|}
\hline \multicolumn{3}{|c|}{ Nuclear hormone receptors } \\
\hline Gene Name & expression change in lim-6/aptf-1 KO mutant & mobility sleep phenotype \\
\hline$n h r-185$ & down by 1,79 seq aptf-1, not in lim-6 & none \\
$n h r-85$ & up by 0,76 seq aptf-1, not in lim-6 & none \\
$n h r-183$ & up by 0,74 ma aptf-1, not in lim-6 & unknown \\
$n h r-23$ & up by 0,68 ma aptf-1, not in lim-6 & none \\
$n h r-198$ & down by 1,65 in aptf-1 (p=0,27), by 2,52 in lim-6 & none \\
$n h r-67$ & no difference in expression but expressed in RIS & none \\
\hline
\end{tabular}

Table 3.3: Nuclear hormone receptors 
(a)

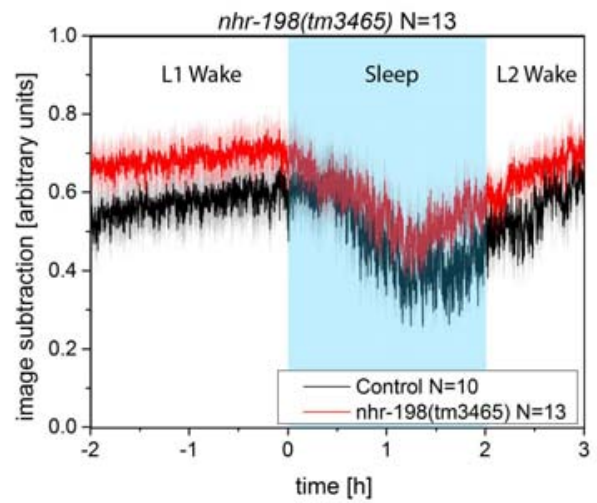

(c)

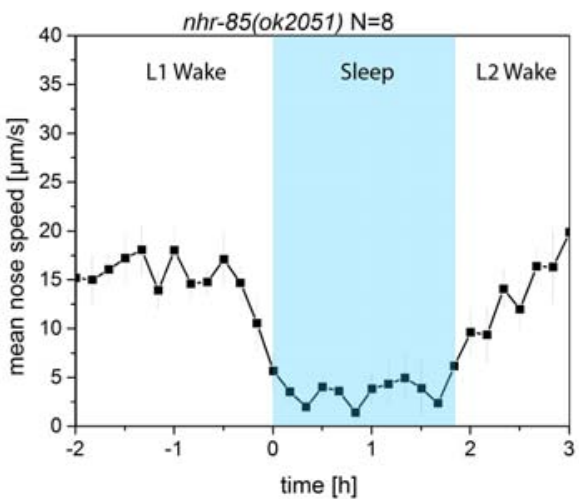

(e)

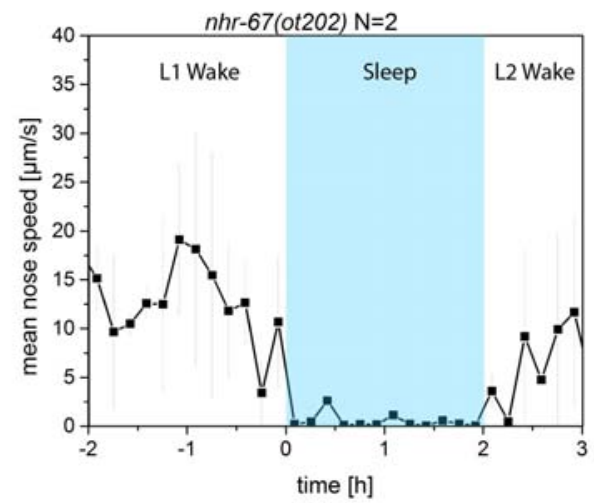

(b)

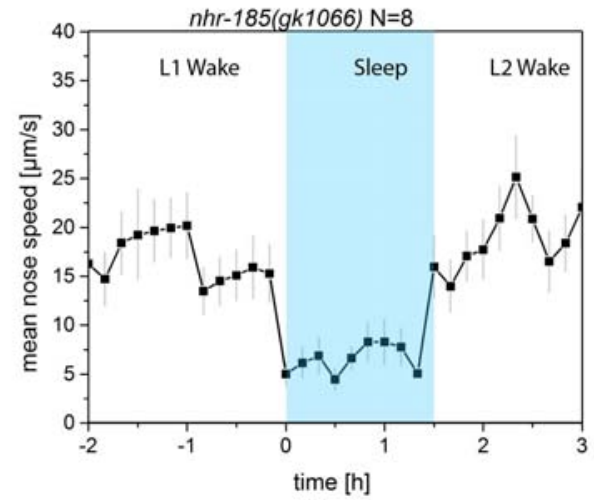

(d)

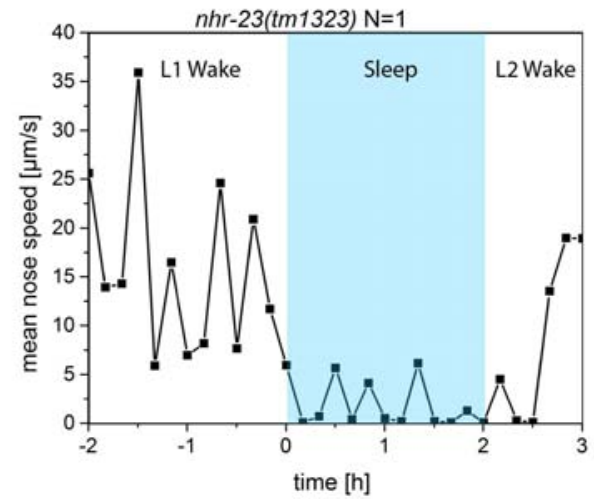

(f)

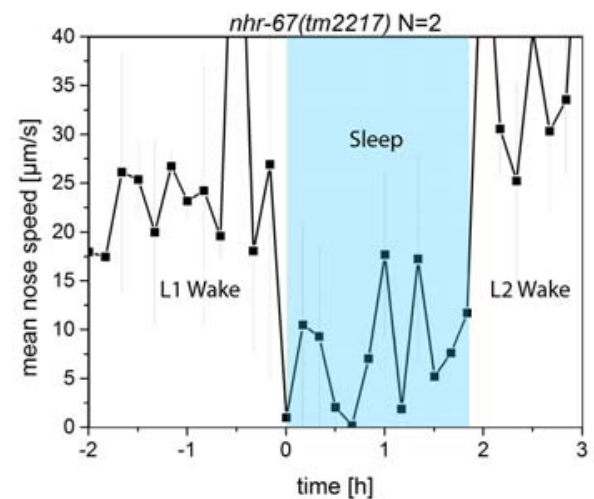

Figure 3.57: Nuclear hormone receptors KO mutants do not show a behavioral sleep phenotype. (a) Mobility, as characterized by image subtraction values, of nhr-198(tm3465) (red) is similar to wild-type control (black). (b-f) KO mutants for (b) $n h r-185$, (c) $n h r-85$, (d) $n h r-23$, and (e, f) $n h r-67$ do not show a behavioral sleep phenotype, as assessed by nose speed. 
trp-2 encodes a transient receptor potential (TRP) channel that has been associated with heat avoidance behavior and its expression in ASE is regulated by lim-6 [93], [94]. Therefore I investigated the sleep phenotype of the trp-2(sy691) KO mutant. However, trp-2(sy691) did not show a behavioral sleep phenotype (Figure 3.58).

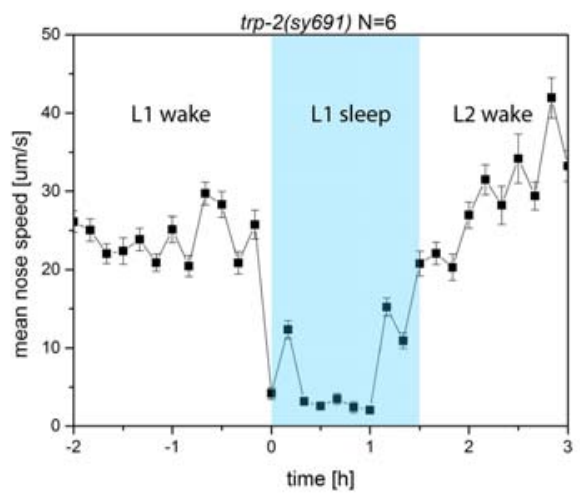

Figure 3.58: Trp-2(sy691) does not show a sleep phenotype. Shown is the nose speed time course averaged for $\mathrm{N}=6$ animals.

\subsubsection{Neurotransmitters}

\section{Dopamine and serotonin signaling}

From the literature it is known that dop-1 and ser-4, a dopamine and a serotonin receptor, are strongly downregulated in RIS in lim-6(nr2073). To find out whether one of these receptors is crucial for the RIS activity at the sleep onset or for the sleep homeostat I investigated $\mathrm{KO}$ mutants for both receptors.

In a first experiment I sleep deprived both receptor $\mathrm{KO}$ mutants with dish tapping and observed their behavior. Similar to wild-type animals, both $\mathrm{KO}$ mutants do react strongly to the waking stimulus for about 30 minutes, but then the reaction to the stimulus rapidly decreases (data not shown). The behavioral response is therefore very similar to wild-type. To verify whether RIS activity patterns differ from wild-type, I crossed both lines into a transgenic line expressing GCaMP 3.35 under the aptf-1 promoter. Undeprived ser-4(ok512) and dop-1(vs100) KO mutants show the typical RIS peak at the sleep onset (Figure 3.59a \& 3.59b). Deprived animals show a long-lasting RIS activation when the reaction to the stimulus decreases (Figure 3.59c \& 3.59d). Therefore I conclude that neither the ser-4(ok512) nor the dop-1(vs100) KO mutant have a sleep or RIS phenotype. It is possible that ser- 4 and dop- 1 receptors redundantly regulate RIS activity. Therefore I created a double $\mathrm{KO}$ mutant for both receptors and crossed it into a RIS GCaMP reporter line under the aptf-1 promoter. The dop-1, ser-4 double KO mutant RIS phenotype does not differ statistically significantly from wild-type either (Figure 3.59e). Four distinct receptors for dopamine and serotonin have been discovered in $C$. 
elegans. To exclude that dopaminergic or serotonergic receptors redundantly generate the RIS activation pattern, a $\mathrm{KO}$ mutant for dopamine/serotonin trafficking is needed. Fortunately in C. elegans $\mathrm{KO}$ mutants for all neurotransmitters except acetylcholine are viable. I investigated $\mathrm{KO}$ mutants that are either defective for dopamine (cat-2(e1112)), serotonin (tph-1(mg280)), or for dopamine and serotonin together (bas-1(tm351)). None of these KO mutants showed a sleep phenotype (Figure 3.60). By crossing the KO mutants tph-1 (mg280) and bas-1(tm351) into the aptf-1 GCaMP transgenic line I measured their RIS activity over the L1-L2 wake-sleep cycle (Figure 3.62). Neither of the mutants showed a RIS phenotype. 
(a)

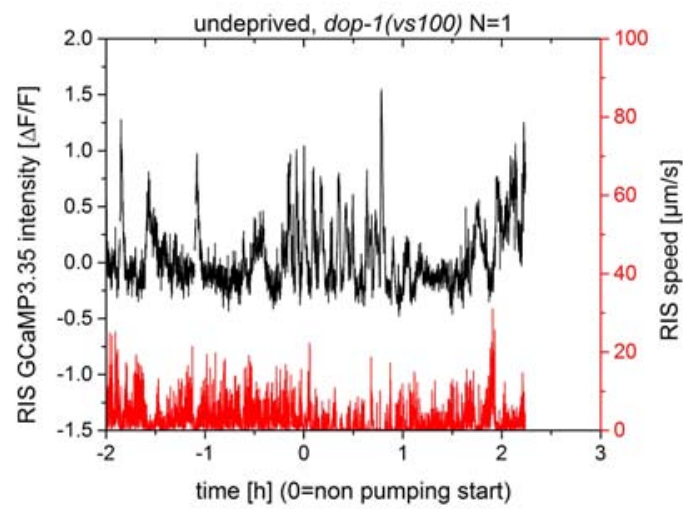

(c)

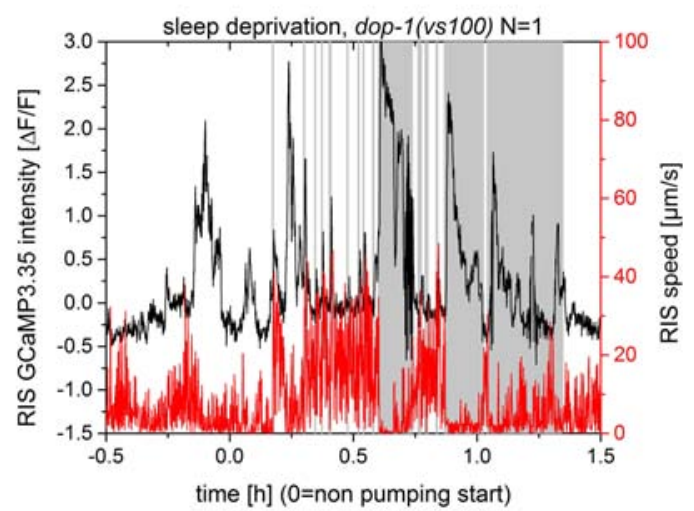

(e)

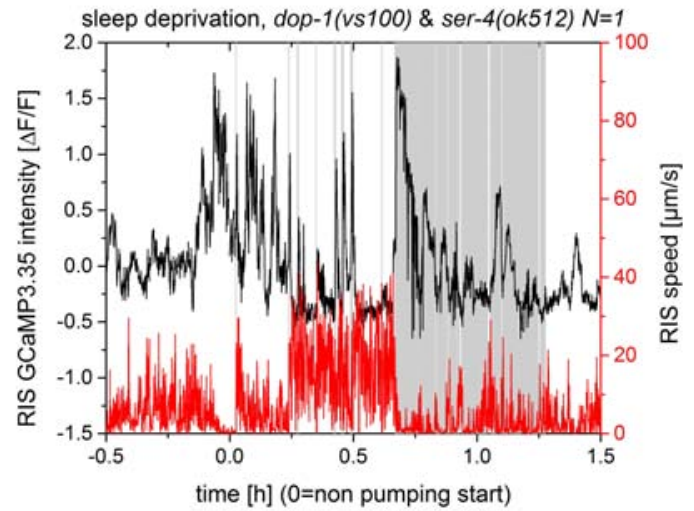

(b)

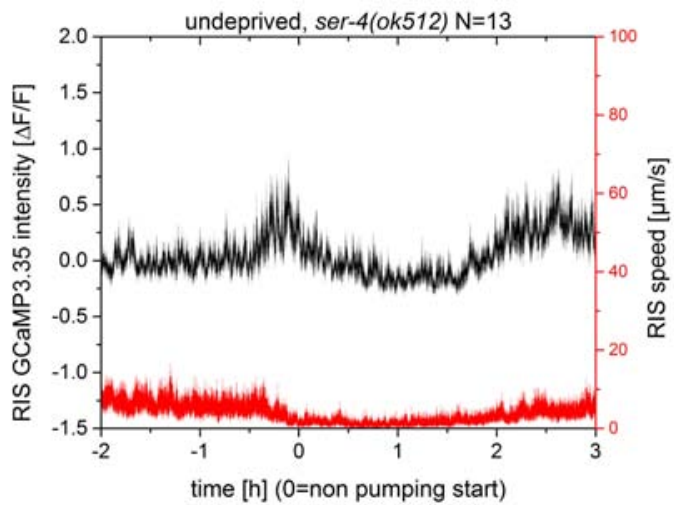

(d)

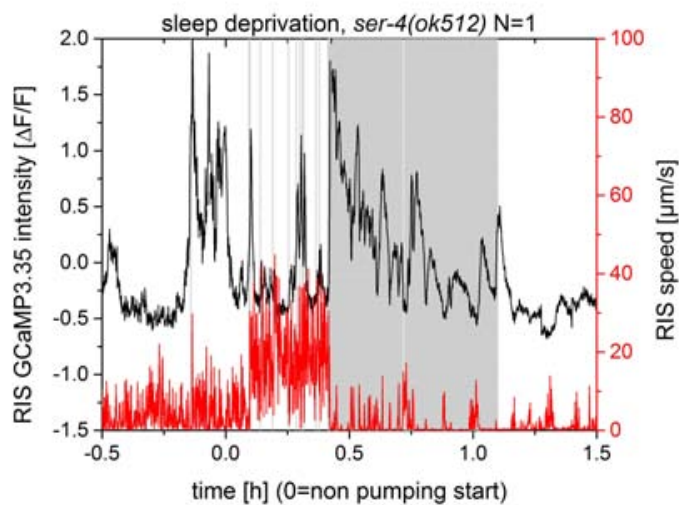

Figure 3.59: KO mutants for a dopamine receptor (dop-1) and a serotonin receptor (ser-4) and the combined double KO mutant do not show a RIS or behavioral sleep phenotype. $(\mathrm{a}, \mathrm{b})$ Undeprived RIS activity and velocity for (a) dop-1(vs100) and (b) ser-4(ok512). (c-e) RIS activity and velocity for sleep deprivation in (c) dop-1(vs100), (d) ser-4(ok512) and (e) dop-1(vs100),ser-4(ok512). Dish tapping stimulation is indicated by gray shading. 
(a)

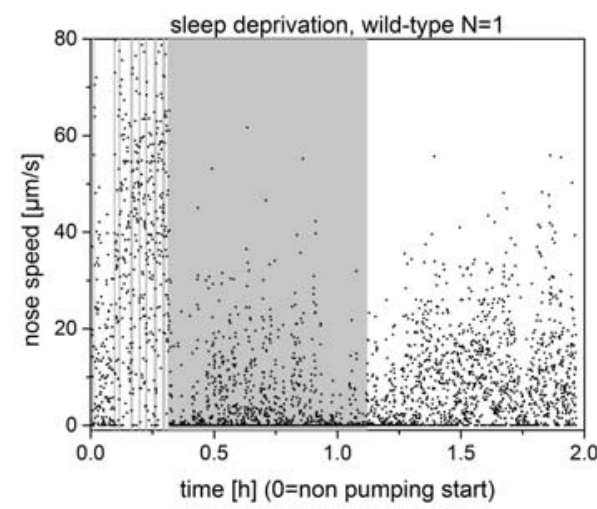

(c)

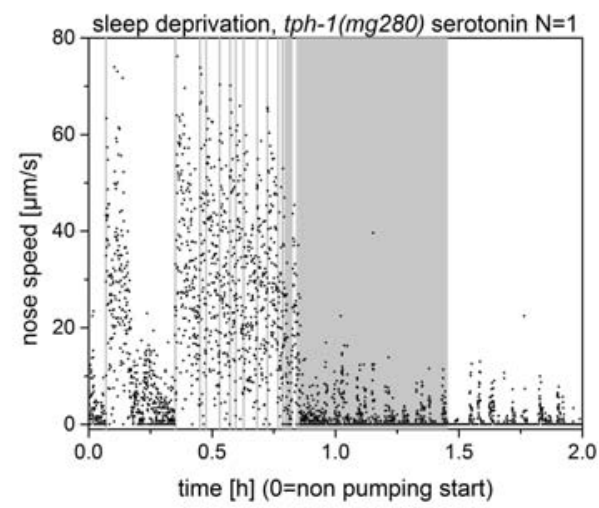

(e)

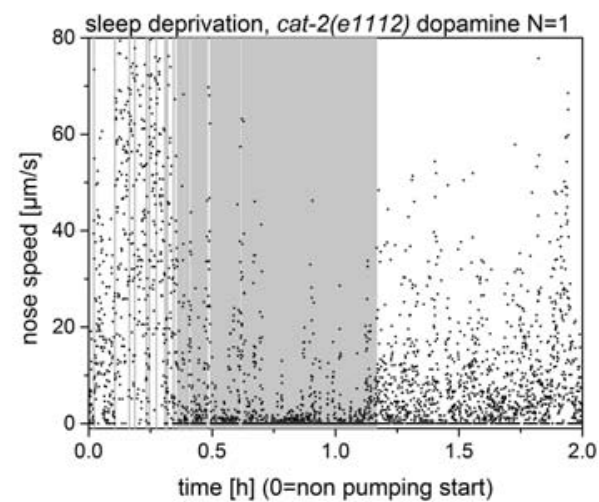

(b)

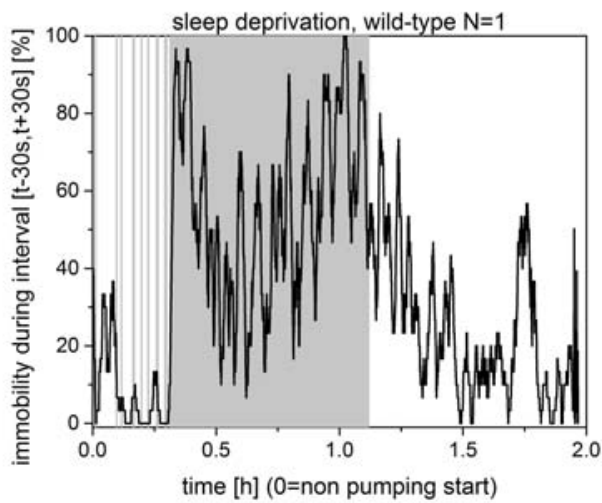

(d)

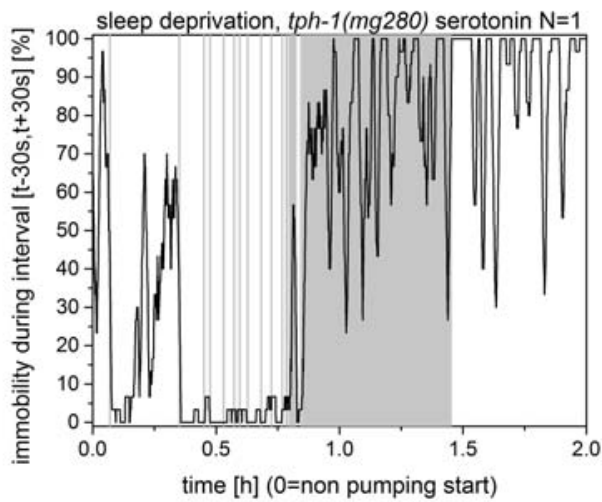

(f)

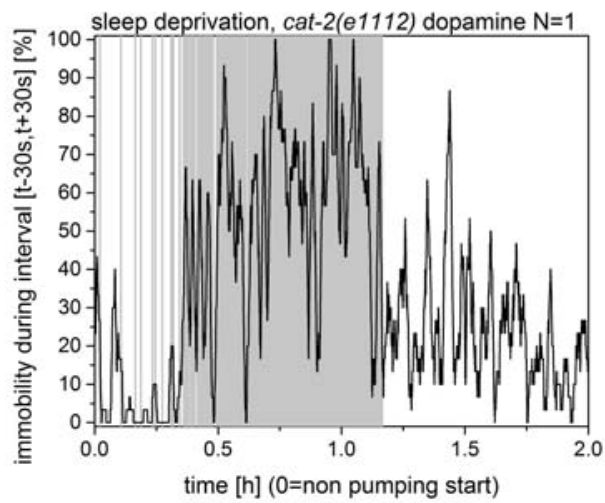

Figure 3.60: $\mathrm{KO}$ mutants for dopamine and serotonin do not exhibit a sleep deprivation phenotype. Shown is $(\mathrm{a}, \mathrm{c}, \mathrm{e})$ nose speed and $(\mathrm{b}, \mathrm{d}, \mathrm{f})$ frequency of immobility over an interval of 60 seconds: (a,b) Wild-type control, (c,d) tph-1(mg280) serotonin $\mathrm{KO}$ mutant and (e,f) cat-2(e1112) dopamine KO mutant. Dish tapping stimulation is indicated by gray shading. 
(a)

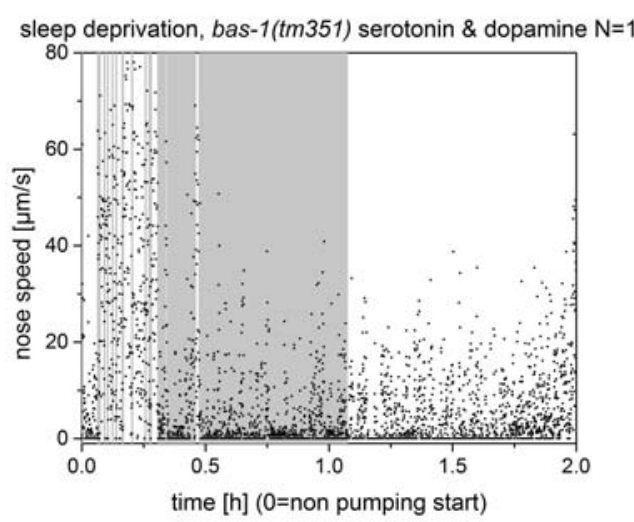

(b)

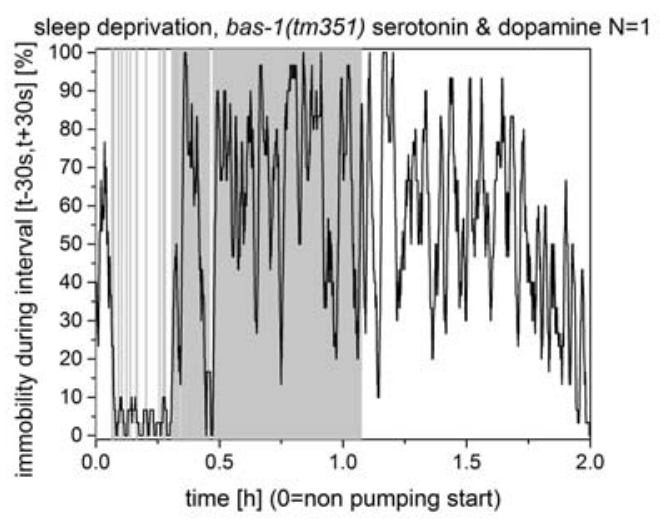

Figure 3.61: The double $\mathrm{KO}$ mutant for dopamine and serotonin does not exhibit a sleep phenotype. bas-1(tm351) dopamine/serotonin KO mutant (a) nose speed and (b) frequency of immobility over an interval of 60 seconds. Dish tapping stimulation is indicated by gray shading. 
(a)

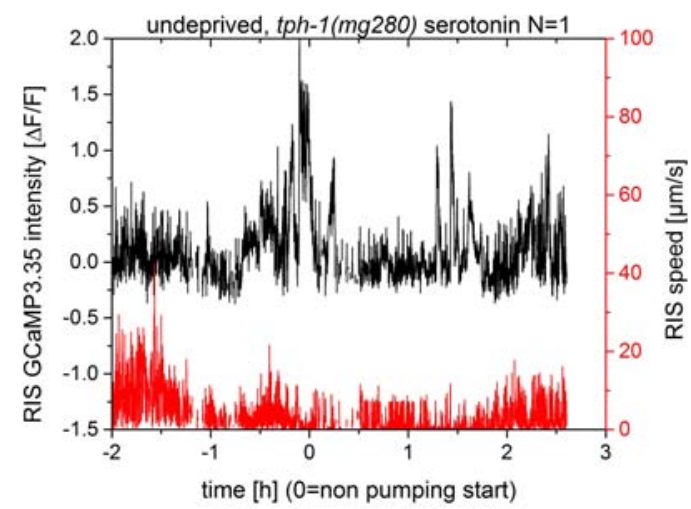

(c)

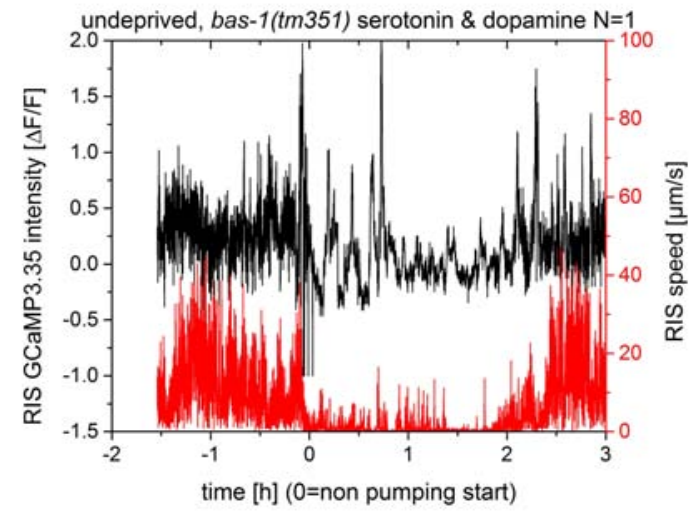

(b)

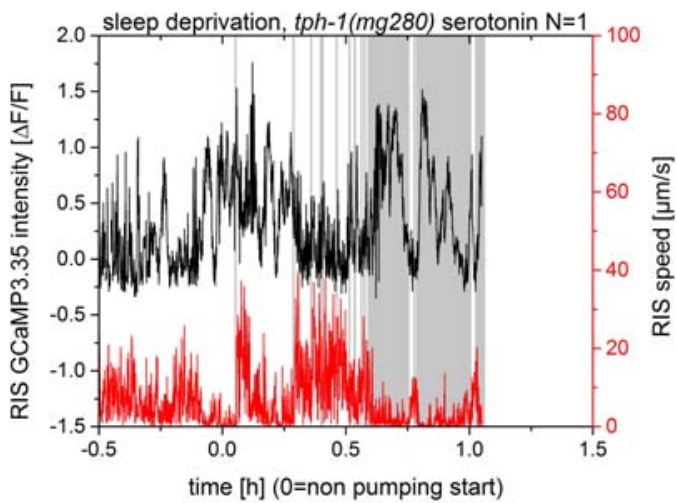

(d)

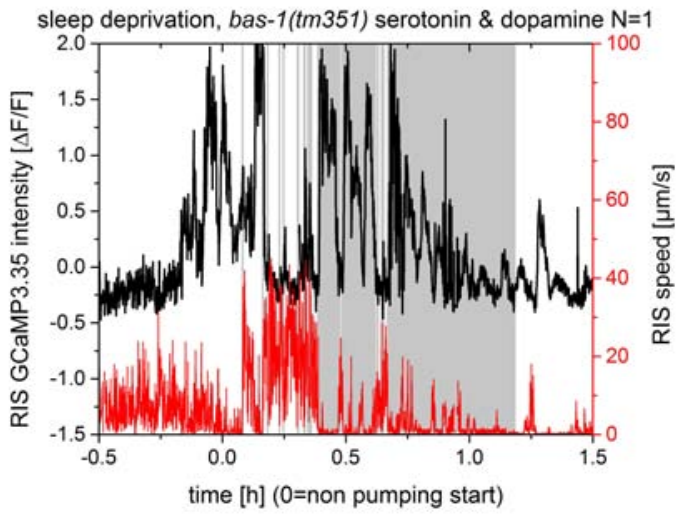

Figure 3.62: Serotonin KO mutant and dopamine/serotonin double $\mathrm{KO}$ mutant do not exhibit a RIS phenotype. (a) Undeprived and (b) sleep deprived tph1(mg280) serotonin KO mutant. (c) Undeprived and (d) sleep deprived bas-1(tm351) dopamine/serotonin KO mutant. Dish tapping stimulation is indicated by gray shading. 


\section{GABA, glutamate and octopamine signaling}

Having excluded dopaminergic and serotonergic trafficking underlying the RIS sleep onset and homeostatic activity, I decided to investigate on the remaining neurotransmitters for which KO mutants exists, namely GABA (unc-25(e156) unc-47(gk192)), glutamate (eat-4(ky5)), and octopamine (tdc-1(ok914)). None of these KO mutants did show a sleep phenotype (Figure 3.63). For the GABA KO mutant unc-25(e156) I additionally investigated RIS activity (Figure 3.64). To sum up, all neurotransmitter KO mutants investigated still show RIS overactivation at the sleep onset and as a response to continued sleep deprivation by dish-tapping. I covered all major neurotransmitters existing in C. elegans except acetylcholine, due to the lack of an acetylcholine KO mutant. 
(a)

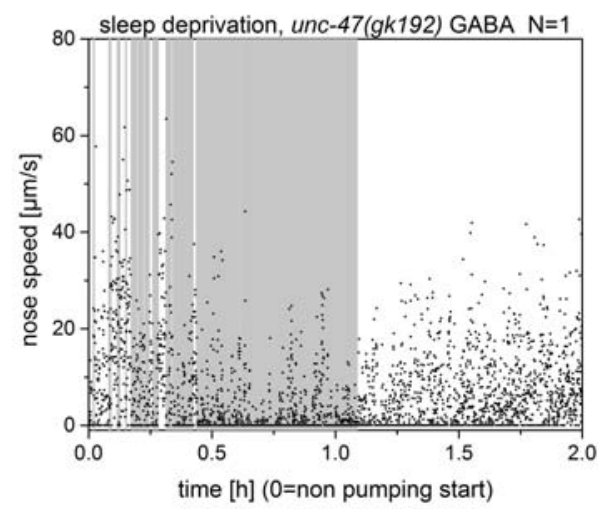

(c)

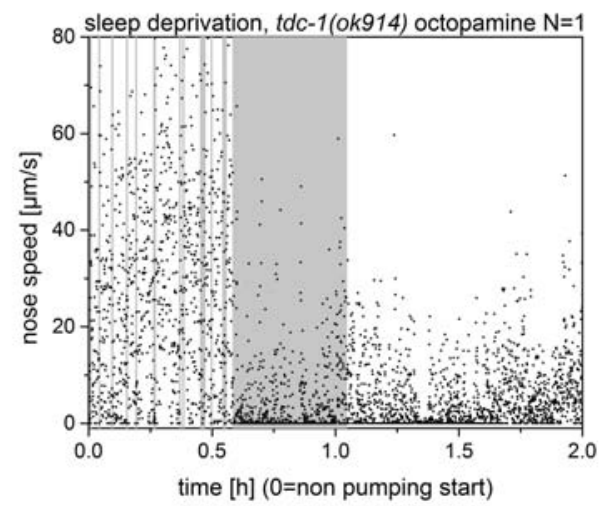

(e)

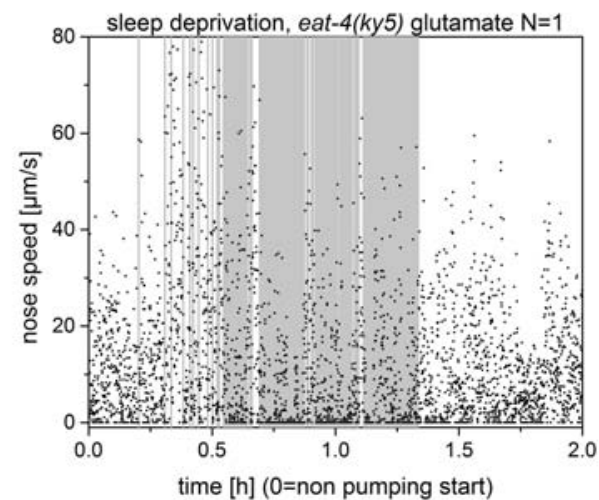

(b)

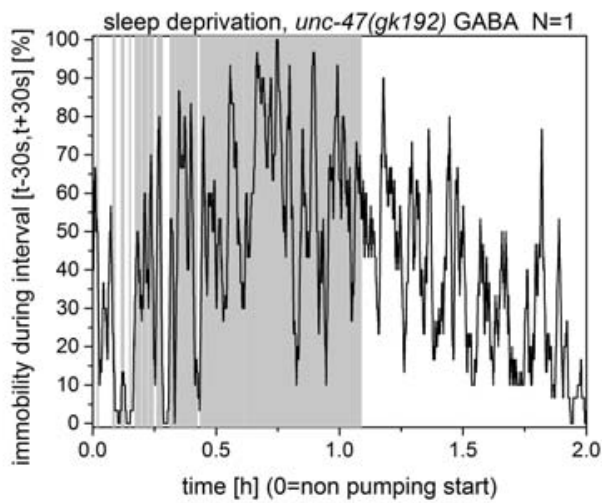

(d)

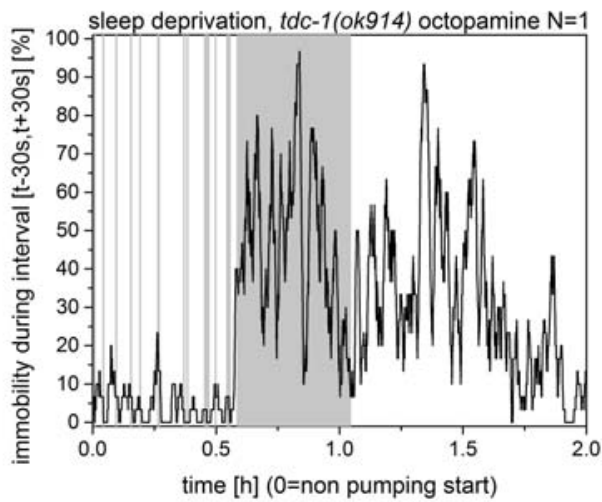

(f)

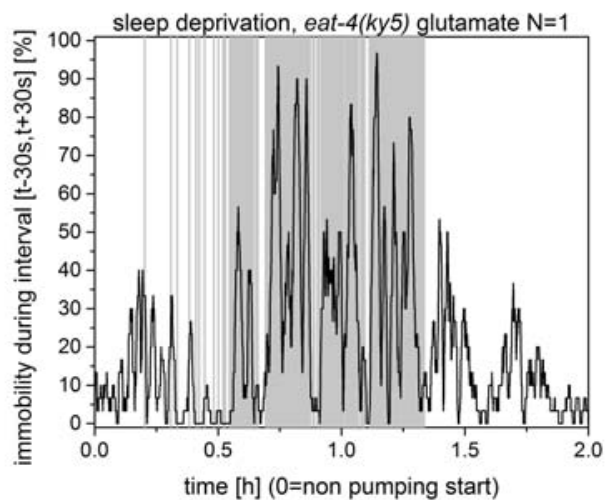

Figure 3.63: $\mathrm{KO}$ mutants for GABA, glutamate and octopamine do not show a sleep deprivation phenotype. Shown is $(a, c, e)$ nose speed and $(b, d, f)$ frequency of immobility over an interval of 60 seconds for a single animal each: $(a, b)$ GABA, $(c, d)$ ocotopamine and $(e, f)$ glutamate. Dish tapping stimulation is indicated by gray shading. 
(a)

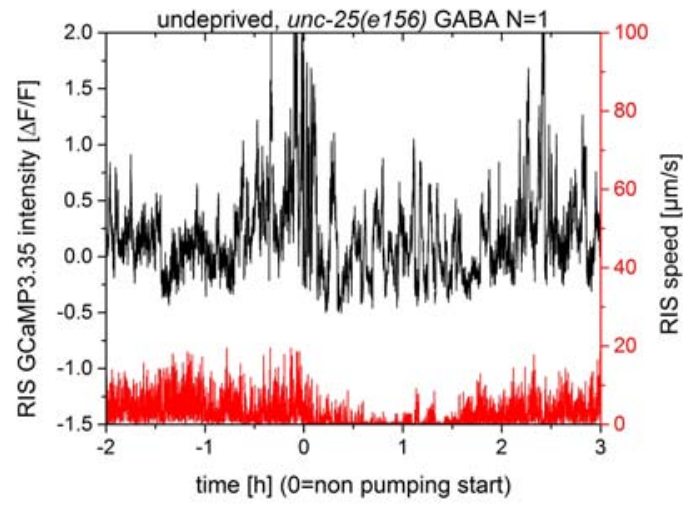

(b)

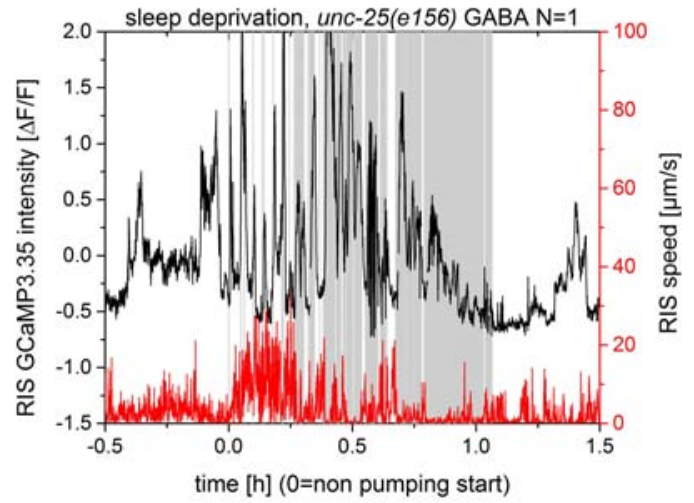

Figure 3.64: The GABA KO mutant unc-25(e156) does not show a RIS sleep phenotype. RIS activity and velocity time course for (a) a single undeprived animal and (b) single sleep deprived animal. 


\subsubsection{Neuropeptides}

As all neurotransmitters investigated do not seem to be crucial for RIS signaling, neuropeptides are another good candidate. Neuropeptidergic trafficking (flp-13 and $n l p-22)$ has been associated with stress induced sleep in C. elegans [51], [50]. In C. elegans 113 neuropeptide genes and more than 250 neuropeptides have been identified [95]. The synthesis of most neuropeptides relies on the proprotein convertase egl-3 [96]. To test whether neuropetidergic neurotransmission is underlying the signaling of RIS it is therefore sufficient to knockout egl-3. egl-3(gk238) KO mutant worms have a sleep phenotype showing quiescence for only $5 \%$ of the non pumping period [40].

Therefore I investigated on RIS activity in egl-3 mutants. egl-3 mutants show long lasting RIS activation peaks not only at the sleep onset but during later parts of sleep as well (Figure 3.65a, 3.65b). The raise in intensity when comparing egl-3(gk238) RIS activity during the second half of sleep to wild-type is striking (Figure 3.65c). RIS activity, averaged over a period of 30 minutes for the mid sleep phase, is increased by $70 \%$ for egl-3(gk238) (Figure 3.65c).

Prolonged sleep deprivation in egl-3(gk238) leads to RIS overactivation but not to immobilization (Figure 3.66a \& 3.66b). This tapping-induced RIS overactivation is statistically significantly higher than for undeprived control animals (Figure 3.66c). Channelrhodopsin activation of aptf-1 expressing neurons in egl-3 $\mathrm{KO}$ mutants does not cause quiescence like in wild-type, but an increase in mobility [40].

The deficiency of RIS to drive quiescence may explain the increased mobility of the egl-3 KO mutant. The RIS sleep onset peak and the increased RIS activity of the egl-3 mutant during sleep indicate that the sleep homeostat upstream of RIS is functional and that neuropeptide signaling is required downstream of RIS. Interestingly, RNA Sequencing for lim- 6 mutants showed that a couple of neuropeptides are downregulated at least 1.5 fold in lim-6 compared to wild-type: flp-28, nlp-6, flp-11, and flp-18. 
(a)

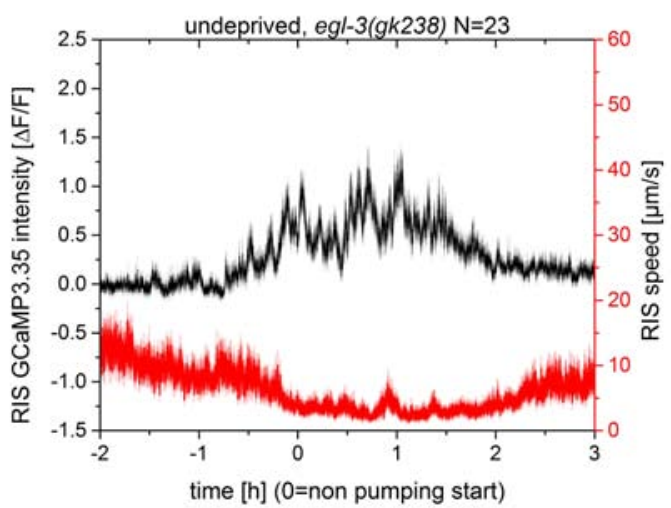

(c)

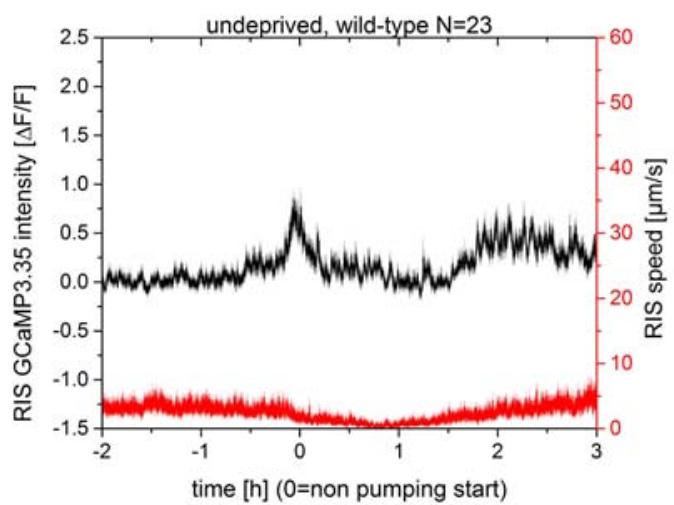

(b)

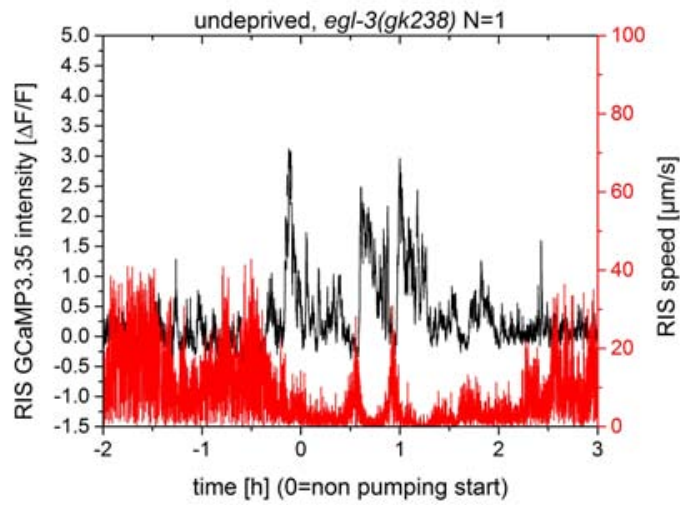

(d)

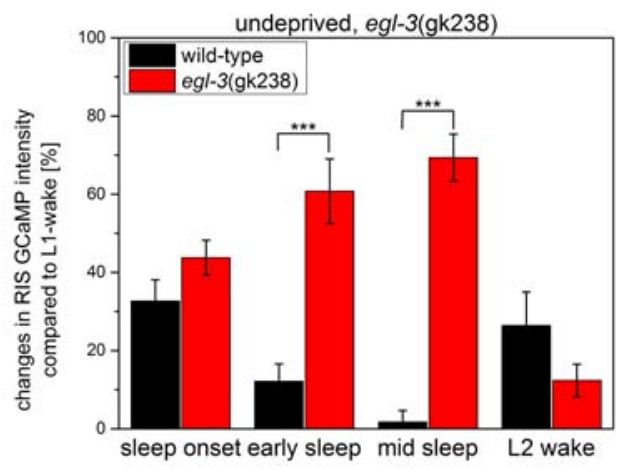

Figure 3.65: The neuropeptide $\mathrm{KO}$ mutant egl-3(gk238) shows increased RIS activity during mid and late sleep. Shown is the undeprived RIS activity and velocity time course of (a) egl-3(gk238), averaged for $\mathrm{N}=23$ animals, (b) representative time course of a single egl-3(gk238) animal (c) wild-type, averaged for $\mathrm{N}=23$ animals. (d) During both early and mid sleep egl-3(gk238) RIS activity is statistically significantly increased compared to wild-type. *** denotes statistical significance with $\mathrm{p}<0.001$, Welch test. 
(a)

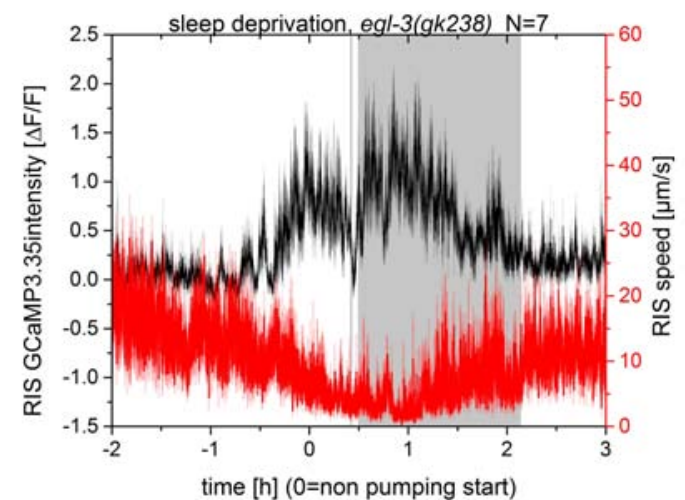

(c)

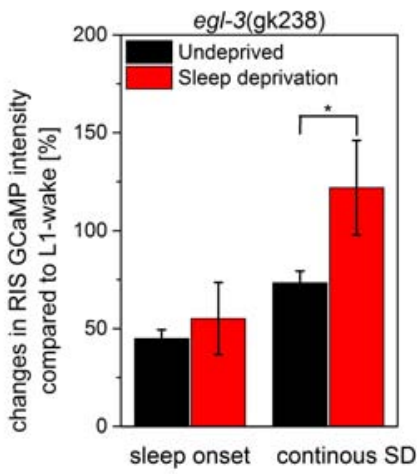

(b)

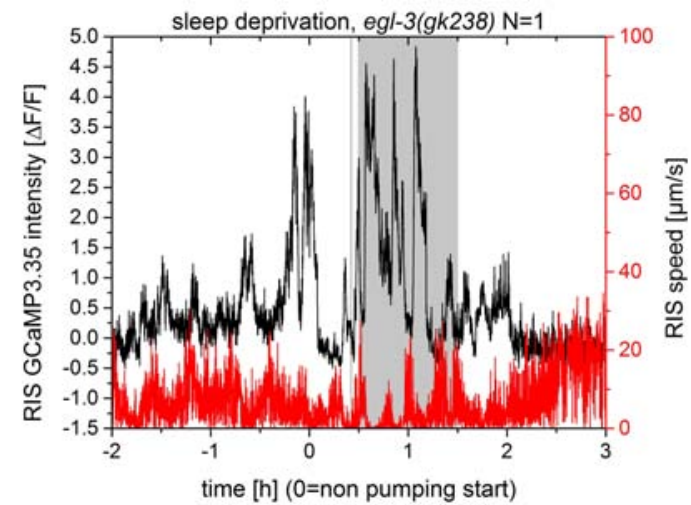

Figure 3.66: Sleep deprived egl-3(gk238) RIS activity and speed (a) averaged for 7 animals and (b) for a single animal. (c) Sleep deprivation of egl-3(gk238) leads to a slight increase in RIS activity. Dish tapping stimulation is indicated by gray shading. ${ }^{*}$ denotes statistical significance with $\mathrm{p}<0.05$, Welch test. 


\subsubsection{Subunits of voltage-gated calcium channels and an inositol trisphosphate receptor}

A screen for C. elegans trans-membrane channel and receptor sleep mutants has been done in our laboratory by Juliane Schwarz. Additional to a basal sleep phenotype she tested for changed arousal thresholds during sleep. As outcome of the screen two mutant strains with a changed sleep or arousal phenotype were found. The first is related to the egl-19 gene, encoding for the alpha-1 subunit of a voltage-gated calcium channel. The other one is related to itr-1, an inositol trisphosphate receptor (personal communication by Juliane Schwarz).

egl-19(n2368) gain of function mutants $(e g l-19(g f))$ have a decreased arousal threshold and preliminary results indicated a lack of RIS overactivation at sleep onset. Therefore I investigated the sleep-homeostat of the egl-19(n2368). I confirmed that RIS activity does not rise at sleep onset (Figure 3.67a).

Comparing absolute GCaMP levels between the egl-19(gf) mutant and wild-type, the former has about three times increased basal RIS activity (Figure 3.67b). This means that the egl-19(gf) phenotype is not a lack of RIS overactivation at sleep onset but an increase of basal RIS activity compared to wild-type. Sleep deprivation initially leads to mobilization of egl-19(gf) and to an increase in its RIS activity (Figure 3.67c). Compared to wild-type the egl-19(gf) mobilizes less and shorter upon tapping during sleep. Mobilization upon tapping during L1 wake is also reduced compared to wild-type, indicating a not sleep dependent general reduction of the response to dish tapping. For another subunit of voltage gated calcium channels, unc-36, preliminary data indicated that the KO mutant (unc-36(e251)) exhibits elevated RIS activity during the entire sleep phase (personal communication Juliane Schwarz). unc-36 is encoding for the $\alpha 2 / \delta$ subunit of a voltage-gated calcium channel. There is a link between unc-36 and egl-19, with the former regulating the voltage dependence, kinetics and conductance of the latter in body wall muscle voltage dependent calcium channels [97]. I could confirm that unc-36(e251) KO mutants are showing an increased RIS activity during sleep (Figure 3.68a). Sleep deprivation in unc-36(e251) using dish-tapping does not lead to any mobilization nor to an increase in RIS GCaMP signal. To conclude whether this is due to the sleep homeostat or to a general mechanosensory deficit in sensing the dish tapping stimulus I did a L1 wake tap control experiment. The L1 wake tap control does not react to tapping at all, indicating that the unc-36(e251) has a mechanosensory deficit. In further experiments other methods of sleep deprivation, for example channelrhodopsin activation of nociceptive neurons, could be used to investigate on the sleep homeostat in unc-36(e251).

The inositol trisphosphate receptor KO mutant itr-1(sa73) exhibits a sleep phenotype that has a shortened non pumping period but additionally shows ectopic sleep phases. Undeprived itr-1(sa73) RIS activity at sleep onset is slightly shorter than for wild-type. For later sleep RIS activity is normally reduced and rises again at the end of the sleep phase (Figure 3.69a). During phases of ectopic sleep RIS activates similar to sleep onset. The L1 wake tap response of itr-1(sa73) is similar to wild-type. RIS does not activate 
(a)

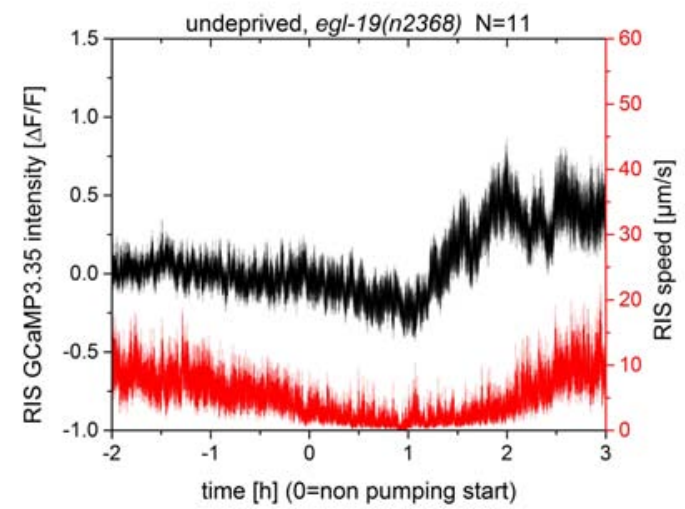

(c)

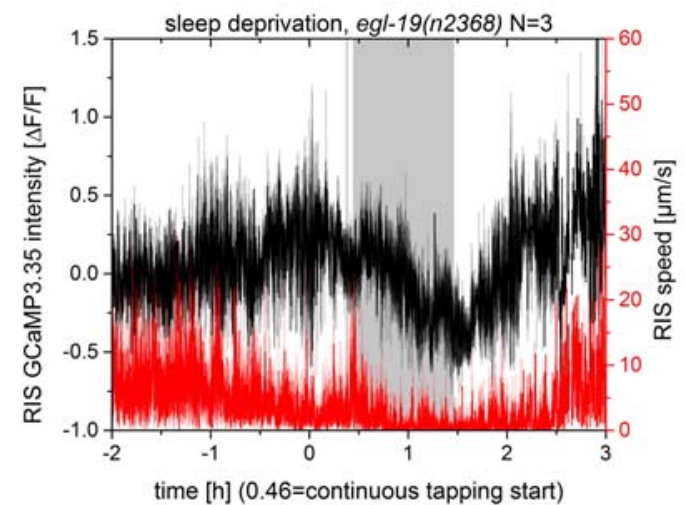

(b)

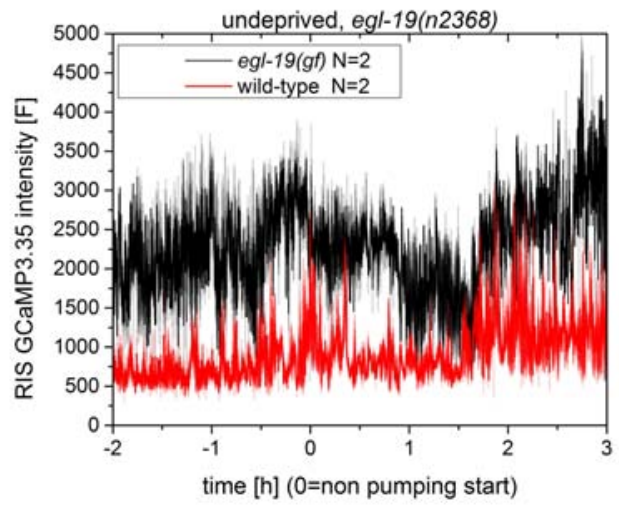

(d)

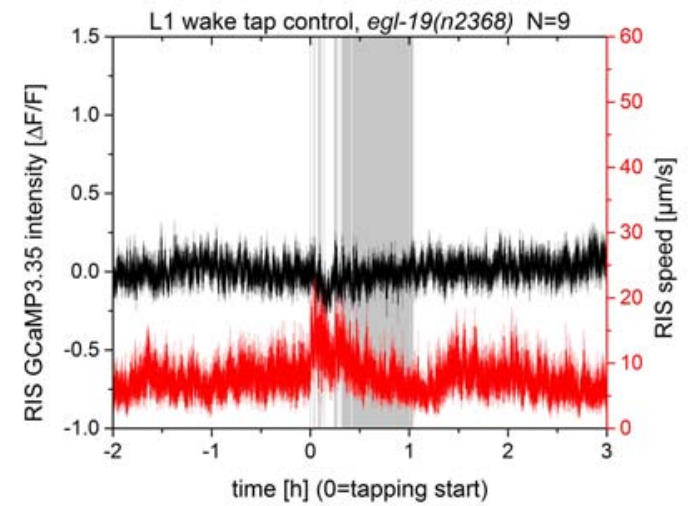

Figure 3.67: egl-19(n2368) has increased basal RIS activity. (a) RIS activity and velocity of undeprived egl-19(n2368) (b) Comparison of absolute RIS GCaMP activity levels of egl-19(n2368) and wild-type. egl-19(n2368) RIS activity and speed for (c) sleep deprivation and (d) L1 wake tap control. Dish tapping stimulation is indicated by gray shading.

upon tapping during L1 wake (Figure 3.69b). Preliminary data indicate that sleep deprivation is less efficient than in wild-type and faster leads to long-lasting RIS activation (Figure 3.69c \& 3.69d). 
(a)

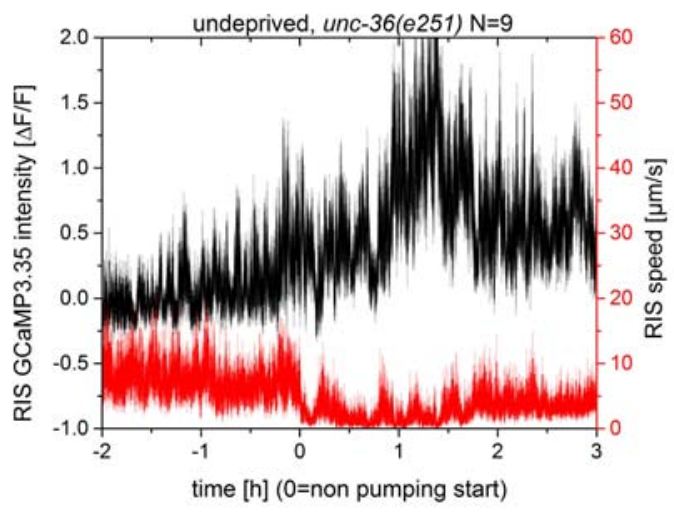

(c)

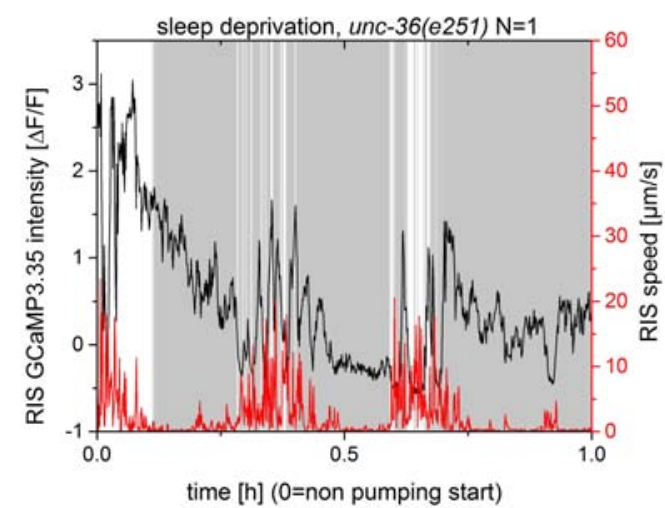

(b)

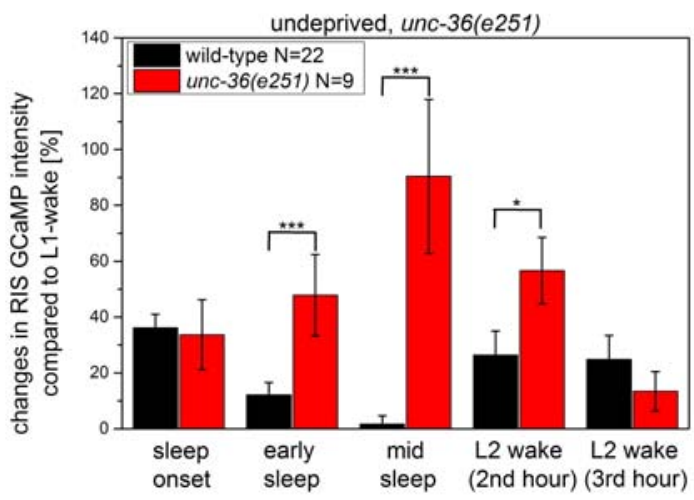

(d)

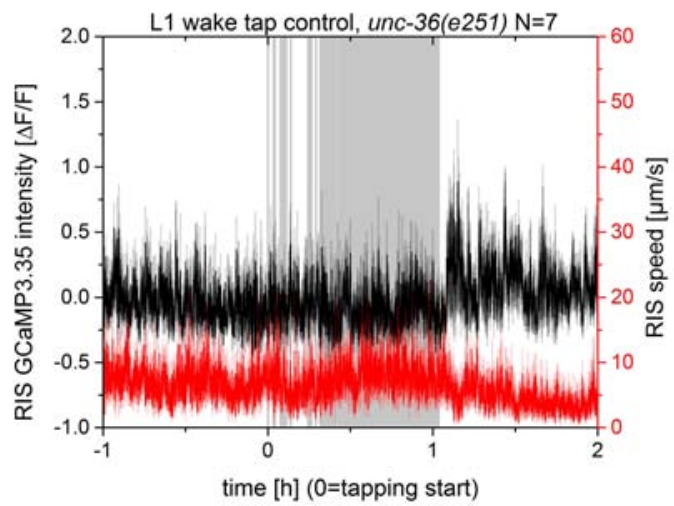

Figure 3.68: The KO mutant for a alpha2/delta subunit of a voltage-gated calcium channel (unc-36(e251)) shows increased RIS activity during sleep. (a) Undeprived RIS activity and velocity time course of unc-36(e251). (b) unc36(e251) activity is statistically significantly increased during the second half of sleep compared to wild-type. (c) Dish tapping during sleep does not lead to mobilization of the animal. (d) L1 wake tap control does not react to tapping either. Dish tapping stimulation is indicated by gray shading. ${ }^{*}$ denotes statistical significance with $p<0.05$ and ${ }^{* * *}$ denotes statistical significance with $\mathrm{p}<0.001$, Welch test. 
(a)

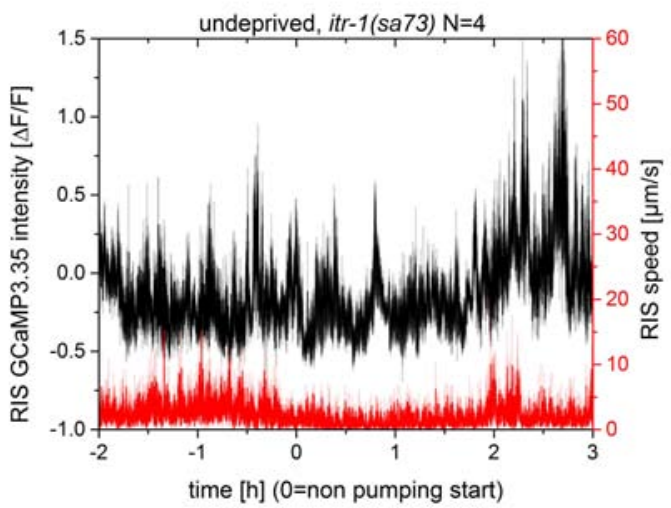

(c)

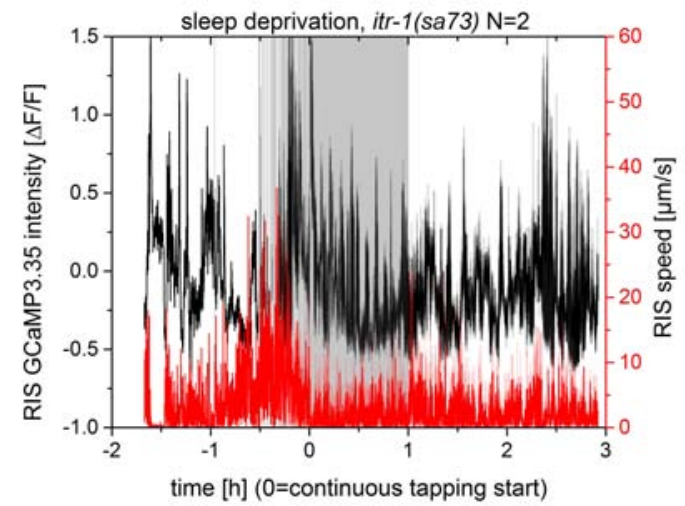

(b)

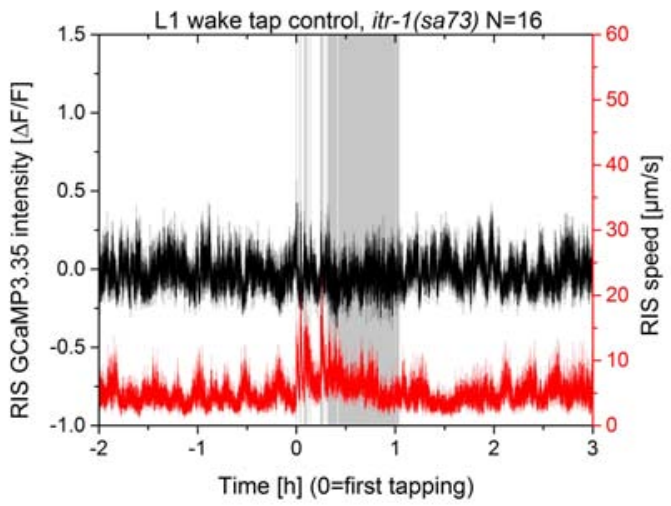

(d)

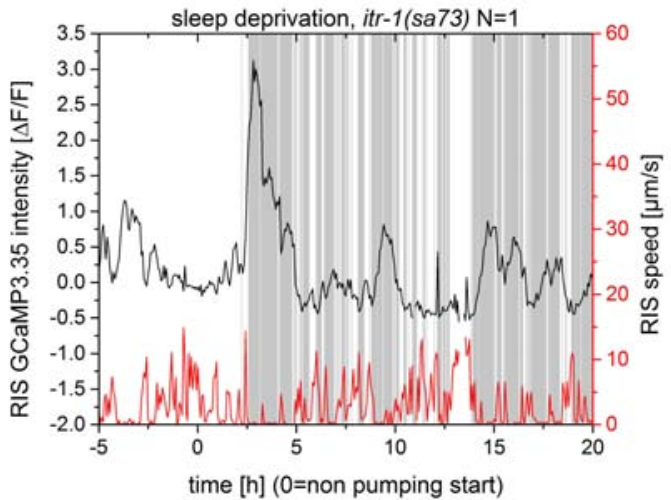

Figure 3.69: Undeprived itr-1(sa73) RIS activity is similar to wild-type, whereas preliminary data indicate that sleep deprivation induced RIS overactivation has a faster time course. itr-1(sa73) RIS activity and velocity time course: (a) Undeprived RIS activity is similar to wild-type. (b) L1 wake tap control mobilize while RIS does not activate. (c), (d) Preliminary data indicate that sleep deprivation is less efficient than in wild-type and faster leads to long-lasting RIS activation. Dish tapping stimulation is indicated by gray shading. 


\subsection{Neuronal regulation of the sleep homeostat besides RIS}

Having shown that RIS activity reflects the sleep homeostat, I wanted to know whether there is more neuronal regulation underlying the sleep homeostat.

\subsubsection{Search for sleep active neurons}

In C. elegans it is possible to observe the activity of all neurons by using a calcium indicator expressed pan-neuronally. I measured neuronal calcium activity over the sleepwake cycle for undeprived worms. By comparing GCaMP3.35/mKate2 ratios I looked for sleep active neurons. Most prominently I observed the RIS sleep onset peak. Furthermore there was one neuron in the tail that activated at the sleep onset, visible in most animals. By comparing the shape, processes and position of this cell, as seen by the mKate2 signal, to the worm-atlas data [98] I identified this cell as probably being PDA. Interestingly PDA is created around this time by a trans-differentiation event of a rectal epithelial cell [99]. PDA activation might be part of the immobility induction, as it is known to innervate the posterior dorsal body wall muscles. aptf- 1 expresses during L1 sleep in only one cell in the tail. Having a similar process and position this neuron might be the very same sleep active neuron. I measured aptf-1 GCaMP3.35 activity and analyzed the activity of this tail neuron (Figure 3.70). Its activity increases at sleep onset, then returns to presleep levels until it starts rising continuously shortly after mid sleep. The sleep onset activity is significantly higher compared to the activity during the time period shortly before and after sleep onset.

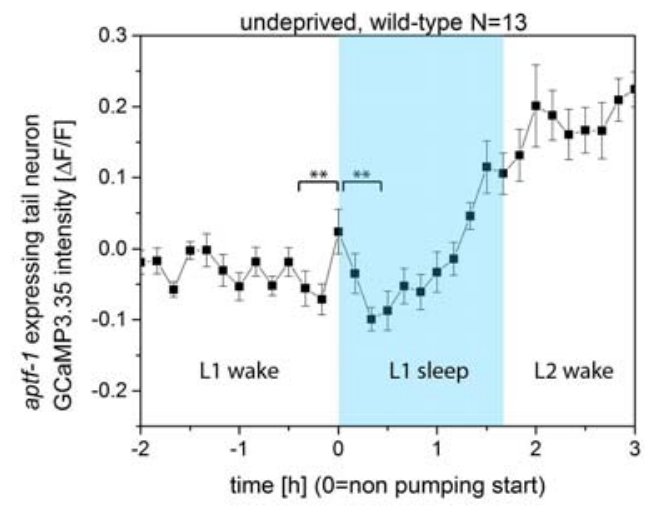

Figure 3.70: aptf-1 tail neuron RIS activity and velocity time course. ${ }^{* *}$ denotes statistical significance with $\mathrm{p}<0.01$, Welch test.

\subsubsection{RIA: A putative wake active neuron}

There is evidence indicating that the sleep homeostat can be described by interactions of wake and sleep promoting neurons [27]. It was reported that ChR2 activation of the in- 
terneuron RIA induces mobility [51]. RIA is therefore a good candidate for a wake promoting neuron. I measured RIA activity using a transgenic line expressing GCaMP3.3 under the $g l r-3$ promoter [67]. During the sleep phase RIA activity decreases by $40 \%$ (Figure 3.71). A decrease of $40 \%$ is more than sixfold as high as the average neuronal activity decrease during sleep, which is about 6\% as measured using GCaMP3.35 ( [39]).

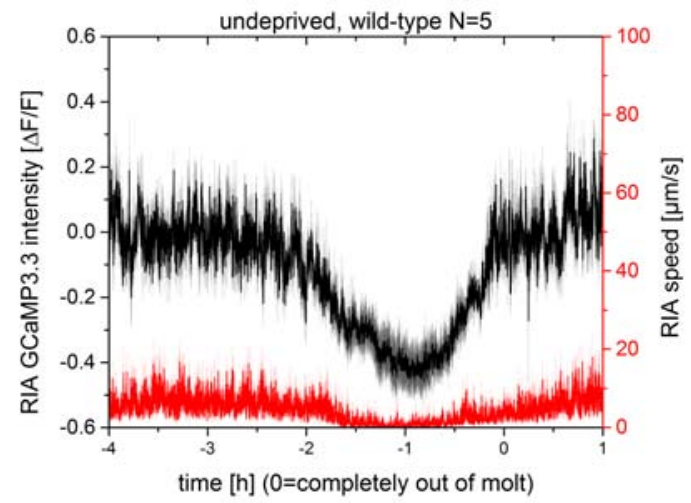

Figure 3.71: RIA activity is downregulated by around $40 \%$ during sleep. Shown is RIA activity and velocity time course averaged for $\mathrm{N}=5$ animals. Animals were aligned by the timepoint when they shed their old cuticle completely (completely out of mold) because the non pumping phase could not be precisely identified.

\subsubsection{Neuronal activity downstream of RIS}

RIS could regulate the activity of the animal by activating or inhibiting other neurons. To investigate on neuronal activity downstream of RIS I used optogenetic methods combined with calcium-imaging. Neuronal density in the head ganglia is extremely high, with neurons being stacked closely together. This makes a identification of single neurons very difficult. To distinguish single neurons in the head ganglia Manuel Zimmer created a transgenic line, expressing GCaMP5K under a pan-neuronal promoter and adding a nucleus localization. I crossed this line into a extrachromosomal transgenic array expressing $\mathrm{ChR} 2$ under the aptf-1 promoter. In a first experiment I activated RIS with ChR2 and measured nuclear GCaMP5K activity. RIS ChR2 activation on Retinal increases the RIS activity around threefold compared to a none Retinal control (Figure 3.72a). In a next step I investigated if and how RIS activation affects neuronal activity. The activity of most neurons remained either unchanged or got reduced. Ventral-chord neurons are most prominently inhibited by RIS. Occasionally head neurons activated together with RIS, but the most consistent activation occurred in the tail (Figure 3.72b). This neuron is not identical to the tail neuron expressing aptf-1, that I hypothesized to be PDA. Future experiments are necessary to identify these neurons that are activated/inhibted by RIS activation. 
(a)

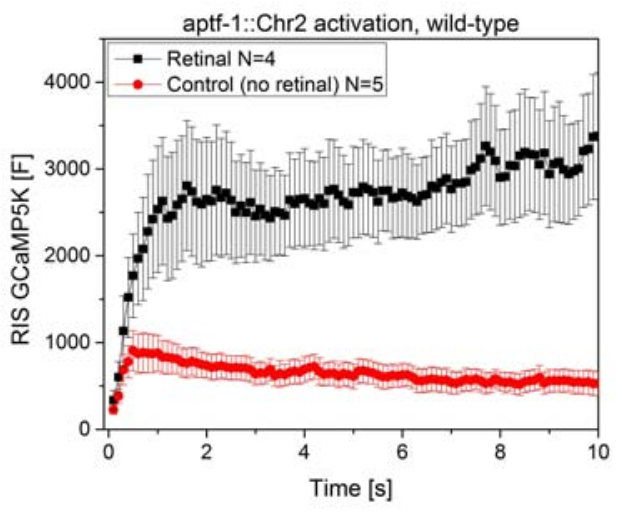

(b)

Comparison of pan-neuronal GCaMP5k activity prior and after ChR2 activation of aptf-1 expressing neurons

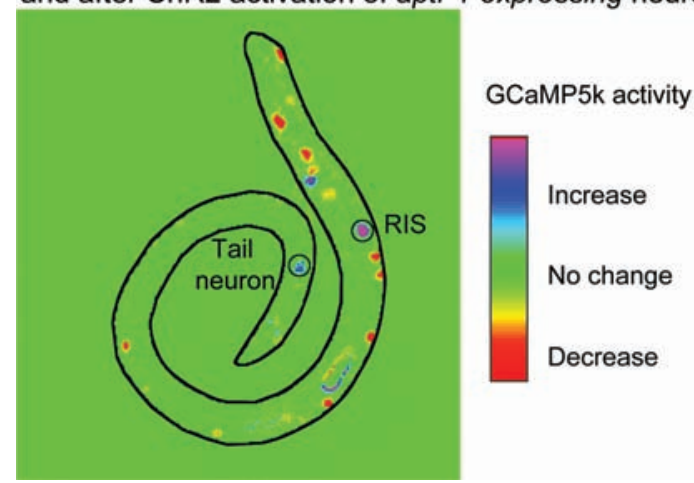

Figure 3.72: ChR2 aptf-1 activation of RIS can be measured using a pan-neuronal GCaMP5K with nuclear localization (a). As a control the experiment was repeated with the same strain without addition of Retinal. (b) Most neurons changing their activity upon aptf-1 ChR2 activation are inhibited. Only an unidentified tail neuron is consistently activated. 



\section{Chapter 4}

\section{Discussion}

\subsection{Development of the " $C$. elegans automated sleep deprivation" setup}

\subsubsection{Sleep detection}

Most established automated tracking algorithms for $C$. elegans behavior are based on the extraction of the worm outline using standard image segmentation techniques. The agarose hydrogel compartments I used contain high contrast bacteria and the mostly transparent worm. This made the extraction of the worm outline a challenging, time consuming task. I established image subtraction as readout for the mobility of the worm that can be used to detect sleep. This year another group introduced image subtraction for quiescence detection [100]. Their experimental setup is based on a microfluidic device, hosting the L4 larvae of C. elegans in a liquid solution [49]. This shows the general applicability of image subtraction for sleep detection in C. elegans, largely independent of the experimental setup used. The "C. elegans automated sleep deprivation" program could therefore possibly also be applied to worms kept in liquid solution. Sleep detection based on image subtraction has some advantages and disadvantages compared to centroid or nose tracking. Image subtraction reflects all movement of the worm and bacteria. Nose or centroid position of the worm could remain unchanged while the posture of the worm changes. However using image subtraction bacteria and worm movement cannot be distinguished. In high moisture conditions bacteria are constantly in motion increasing the image subtraction value. Basing the detection of sleep on immobility has the disadvantage that in some occasions the worm appears quiescent but slowly drifts through the chamber. This passive movement is detected as wakefulness. An automated nose detection algorithm based on my preliminary work is shortly before finalization. An integration of the nose speed to identify sleep could be implemented in a future version of the "C. elegans automated sleep deprivation" program. The nose, while actively foraging being the only mobile part of the worm, would improve the distinction of low mobility feeding periods from sleep.

\subsubsection{Sleep deprivation}

Sleep deprivation in C. elegans was done using manual mechanic stimulation with an eyelash and forced swimming (in a constantly vortexed liquid) [36], [84], [41]. These 
methods have in common that they require the manual administration of the deprivation stimulus. I introduced two automatized sleep deprivation techniques, ChR2 activation of neurons and mechanical stimulation by dish tapping. For both methods I showed that these can be successfully used to deprive sleep in C. elegans. Dish tapping has the advantage of being not physically harmful and highly reproducible. The electromagnet is always accelerating the piston to the same speed. The main limitation for reproducibility in this setup is the wear of the spring over time. Dish tapping has the limitation that the deprivation stimulus can not be applied independently to animals that are filmed simulatenously.

ChR2 activation of neurons requires blue light illumination that is toxic to the animal. I tested two red shifted channelrhodopsin variants, C1V1 and Chrimson. Activation of either variant in mechanosensory or nociceptive neurons did not lead to a strong behavioral response and was therefore not suited for deprivation. ReaChR, a recently developed red-shifted ChR2 variant [73], shows promising first results regarding the activation of neurons in C. elegans (personal communication Juliane Schwarz). Nontoxic long lasting green light activation would allow persistent activation of ReaChR. In future experiments one should test whether ReaChR activation of neurons can be used for sleep deprivation. ReaChR activation would offer the advantage to simultaneously deprive more than one animal at the same time. A disadvantage of ReaChR is its wide action spectrum. While simultaneously imaging GCaMP with blue light one would have to carefully control for undesired activation of ReaChR expressing neurons.

\subsection{Sleep deprivation shortens sleep duration and yields persistent increased locomotion past the deprivation period.}

To my knowledge, I am the first to report sleep deprivation in the L1 C. elegans larvae. I could show that partial deprivation of the L1 sleep shortened the sleep length significantly by $9.3 \%$, as measured by non pumping time. This is partly in agreement with findings of sleep deprivation in L4 that does not lead to a prolongation of sleep length [36]. Sleep in C. elegans larvae is strongly coupled to development, which could restrict the timing of sleep. The period homolog lin- 42 is needed in C. elegans for the correct developmental timing [44]. I showed that sleep deprivation of lin-42 $\mathrm{KO}$ mutants does not lead to a prolongation of the sleep phase. This means that either lin-42 $\mathrm{KO}$ alone is not sufficient to abolish a developmental restriction for the sleep timing, or that $C$. elegans compensates sleep loss by higher sleep intensity alone. This would be in concordance with finding in cockroach, honey bee and tilapia that do not show an increase in sleep duration following sleep deprivation (see review [17]). It is scientific consensus that sleep homeostasis is mainly adjusting the sleep intensity and only to a lower degree the sleep timing.

I found that sleep deprivation induces an increase in mobility that persists also during consecutive undeprived sleep. The fraction of quiescence and the quiescence bout du- 
ration are decreased following sleep deprivation compared to control. This disagrees with finding in L4 sleep, where the fraction of quiescence and the quiescence bout duration are increased following sleep deprivation [36]. This might be due to differences between the sleep phases or the deprivation methods. Raizen et al. increased the strength of a manually applied mechanic stimulus, whereas I always apply the same stimulus strength. In accordance with Raizen et al. I showed that homeostatic effects following sleep deprivation are mainly restricted to a short time window following sleep deprivation.

\subsection{Homeostatic regulation of sensory responsiveness}

I showed that mechanosensory neuron responsiveness is lowered in sleep following persistent sleep deprivation. This is the first direct finding of homeostatic downregulation of single neuron responsiveness to an arousal stimulus. It is in agreement with findings in zebrafish, where it was demonstrated that a group of neurons shows a robust response to light stimulation that is inhibited by melatonin treatment, mimicking prolonged wakefulness [101]. In future experiments, one should uncouple the deprivation and arousal stimulus. This could be done using a red shifted channelrhodopsin variant for deprivation and dish tapping for arousal stimulation. Another possibility is to use strong dish tapping for deprivation and mild dish tapping to assay for homeostatic effects. For this purpose I conceived a device containing two dish tappers that can be controlled independently of each other, see appendix C.

\subsection{RIS is reflecting the sleep homeostat}

\subsubsection{Sleep promoting neuron}

I showed that the interneuron RIS is reflecting the homeostat. It is actively driving quiescence at sleep onset and as a response to sleep deprivation. Sleep promoting neurons of the VLPO are mostly silent and get specifically activated at sleep onset in mammals [25], [27]. There is evidence indicating that the regulation of sleep and waking is based on mutual regulation of sleep and wake promoting neurons [102]. C. elegans with all its neuronal connections known and the simplicity to measure neuronal activity in vivo is the perfect system to dissect the circuitry underlying the neuronal sleep homeostat.

\subsubsection{RIS overcomes arousal promoting neuronal signaling}

I identified RIS as the neuronal mechanism suppressing the neuronal arousal promoting circuits to induce quiescence after prolonged sleep deprivation. Calcium imaging of mechanosensory neuron activity during sleep deprivation by dish tapping showed that the tapping stimulus is still sensed while the animal stops responding and immobilizes. Similarly, the thalamic gate in mammals shuts off sensory information from being processed by the hypothalamus during sleep. 


\subsubsection{RIS circuitry}

I showed that activation of RIS using ChR2 is mostly downregulating neuronal activity. Ventral-chord neurons are most prominently inhibited by RIS. They are possibly motorneurons related to the immobilization of the animal. Only a single tail neuron is consistently activated. Future experiments are necessary to identify these neurons. The most reliable method for identification is the comparison with expression patterns of known markers. By crossing these markers into the transgenic line of interest it is possible to conclude its identity by overlaying the fluorescence signals. Further, RIS activation/inhibition experiments combined with calcium imaging are needed to elucidate the circuitry of RIS. Different wavelengths should be used for RIS activation/inhibition and calcium imaging for a more precise quantification of the changes of neuronal activity.

There is preliminary evidence indicating that an isolated RIS, obtained by ablating its process, shows no apparent activity differences between sleeping and waking animals (personal communication Michal Turek). This is a strong argument in favor of neuronal control of the sleep homeostat upstream of RIS. It is known that only 11 neurons are upstream of RIS: AIB, AVJ, CEP, DB, OLL, PVC, RMD, RIM, SDQ, SMD, and URY [103]. Correlating their neuronal activity at sleep onset to RIS would probably allow the identification of upstream sleep regulatory neurons. To do so I suggest to first use a pan-neuronal GCaMP line to find the most promising candidates. For these neurons I would then create a cell specific (or subset specific) GCaMP line and cross it into the aptf-1 GCaMP line to correlate their activity with RIS.

\subsubsection{Locomotion origin of the sleep homeostat}

I demonstrated that RIS also has a more general, sleep unrelated, function on the homeostatic regulation of locomotion. High mobility correlates with low RIS activity, whereas high RIS activity correlates with low mobility not only during sleep, but during wake as well. I showed that RIS dampens tapping-induced mobility by activating similar to ALM, both in sleep and in wake. The fact that RIS is controlling both the balance between mobility and immobility and the sleep homeostat suggests a related evolutionary origin.

\subsubsection{Other mechanisms besides RIS that regulate sleep homeostasis in C. elegans}

I showed that the reduced responsiveness after prolonged sleep deprivation is not induced by active regulation through RIS. Furthermore RIS ablated animals still show a small mobility decrease during the sleep phase [40]. This implies that there must be other mechanisms besides RIS that regulate sleep homeostasis. These unknown mechanisms could be addressed by investigating the RIS phenotype of known sleep mutants. If their sleep phenotype is not due to altered RIS signaling, analyzing their expression 
4.5. Molecular and genetic mechanisms underlying the sleep homeostat

pattern and function is a starting point to discover novel mechanisms important for sleep homeostasis.

\subsection{Molecular and genetic mechanisms underlying the sleep homeostat}

I could identify several molecules that are involved in regulation of RIS activity during sleep. However, none of these molecules were crucial for the sleep homeostat. Transcription factors regulating protein environment in RIS and having a sleep phenotype were a starting point to address molecular regulators of RIS activity.

\subsubsection{Transcription factors and their role in the sleep homeostat - aptf-1 and lim-6}

aptf-1 knockout mutants were reported to have a strong sleep phenotype that is related to RIS [40]. My data indicate that aptf-1 mutants have a slightly reduced RIS sleep onset peak, increased RIS activity during sleep and possibly a decreased RIS activation in response to sleep deprivation. This altered RIS activity let me conclude that aptf-1 is important for the sleep homeostat.

I discovered that a knockout of the transcription factor lim- 6 induces a light sleep phenotype. However, its RIS activity is similar to wild-type. lim-6 mutant animals show a sleep onset peak of RIS activity and an increase in RIS activity in response to sleep deprivation. I showed that lim- 6 is regulating aptf-1 expression in RIS, suggesting that the lim-6 sleep phenotype is due to regulation of aptf-1 expression in RIS. However a sleep phenotype rescue was not obtained for a limited expression of lim- 6 rescue constructs to a few neurons including RIS. Nevertheless, a rescue of the lim- 6 sleep phenotype was successful using a lim- 6 full promoter rescue line (carrying additionally the rol-6 $\mathrm{KO}$ mutation). This can only be explained by either the constructs for the lim- 6 rescue not being valid or the lim- 6 phenotype not being due to RIS regulation. To exclude a malfunction of our computer build lim- 6 rescue constructs, one should repeat the rescue experiment with limited expression using the rescue construct that was used successfully with the full $\mathrm{lim}-6$ promoter. To exclude that $1 \mathrm{im}-6$ is required in non-neuronal tissue to rescue the sleep phenotype, one should use a lim- 6 rescue construct expressed under the lim-6up promoter [89]. This might be worthwhile, as there is evidence linking non-neuronal tissue to sleep and sleep homeostasis in C. elegans [41].

To find out how aptf-1 and lim-6 affect sleep and/or the sleep homeostat I investigated possible target genes that were downregulated in the lim- 6 or aptf-1 mutant as was shown by transcriptional profiling.

\subsubsection{Nuclear hormone receptors}

Nuclear hormone receptor genes were a good candidate for controlling sleep regulation, as they are known to influence development and metabolism [91]. Therefore I 
investigated nuclear hormone receptors genes that were downregulated in either the lim- 6 or aptf-1 mutant. However none of these nuclear hormone receptors genes exhibited an obvious sleep phenotype. As I only checked a small number of the 284 existing nuclear hormone receptors in C. elegans [91], I cannot exclude that nuclear hormone signaling is at the base of the sleep homeostat.

\subsubsection{Neurotransmitter signaling}

I investigated which neurotransmitters are required for the sleep homeostat to be functional. I covered the major neurotransmitters in C. elegans, namely serotonin, dopamine, GABA, glutamate, and octopamine. I did not investigate acetylcholine, due to the lack of a viable acetylcholine knockout mutant.

All KO mutants investigated still show sleep homeostasis. Similarly to wild-type, there is RIS overactivation at the sleep onset and as a response to continued sleep deprivation by dish-tapping.

\subsubsection{Neuropeptide signaling and its function on the sleep homeostat}

I showed that the proprotein convertase egl-3 KO mutant, that is deficient in the synthesis of neuropeptides, shows RIS overactivation in the mid and late sleep phase. This together with the evidence that RIS activation does not drive immobility in the egl-3 mutant [40] implies that neuropeptide signaling is required downstream of RIS. RIS overactivation during sleep is either due to a negative feedback loop or indicates that egl-3 signaling is required by upstream neurons, which inhibit RIS. To conclude on this one should do a RIS specific egl-3 rescue of the egl-3 KO mutant. A lack of the RIS overactivation would then indicate that a negative feedback loop is underlying the egl3 RIS overactivation during sleep. The fact that the egl-3 mutant has an only slightly increased RIS onset peak and also shows RIS overactivation in response to tapping indicates that homeostatic regulation upstream of RIS is still largely intact. Future experiments should investigate the role of the neuropeptides $n l p-6, f l p-11, f l p-18$, and $f l p-28$ that I found to be downregulated in lim-6 KO mutants.

\subsubsection{Ion channels and their function on the sleep homeostat}

Manipulating overall ion channel activities leads to strong changes in sleep intensity and duration, as discussed in the review [33]. This is probably due to altered neuronal depolarization inducing altered excitability. Recently it has been shown that ion channel modulation specifically in sleep promoting neurons is at the base of sleep homeostasis in Drosophila [104]. I could verify that the inositol trisphosphate receptor itr-1(sa73) KO mutant has a reduced RIS sleep onset peak and there is first evidence that the RIS mediated homeostatic response to sleep deprivation is altered. Furthermore it exhibits ectopic sleep phases that are accompanied by RIS activity peaks. ITR-1 is known to generate a pacemaker rhythm for defecation by activating the motorneuron DVB via neuropeptide signaling [105]. A similar mechanisms could be at the base of 
the locomotion control by RIS activation. Future experiments are necessary to elucidate if this receptor has a crucial function for sleep.

The calcium channel $\alpha 2 / \delta$ subunit unc-36 KO mutant shows RIS overactivation in the mid and late sleep phase. Sleep deprivation using dish tapping was not successful because this mutant is mechanosensory deficient, thus not sensing the mechanical stimulus. In future experiments one should sleep deprive unc-36 mutants by activation of nociceptive neurons, preferably by red shifted channelrhdoposins, to investigate on their sleep homeostat.

\subsection{Conclusion}

Together, this work has revealed new insights in sleep homeostasis and its regulation: First, I showed that the activity of the interneuron RIS reflects the sleep homeostat. Overactivation of RIS induces quiescence at sleep onset and in response to sleep deprivation. RIS activation suppresses the neuronal arousal promoting circuits, even while the deprivation stimulus is still sensed.

Second, I identified several molecules that are important for the regulation of RIS during sleep. Proteins as diverse as neuropeptides, ion channel components and transcription factors regulate the activity of RIS. Mutants deficient for neuropeptide synthesis, egl-3, show strong RIS overactivation during sleep. Ion channel signaling by the inositol trisphosphate receptor itr-1(sa73) and the calcium channel subunit unc-36 is required for wild-type RIS activity during sleep. aptf-1 knockout mutants have a slightly reduced RIS sleep onset peak, increased RIS activity during sleep and possibly a decreased RIS activation in response to sleep deprivation. The neurotransmitters serotonin, dopamine, GABA, glutamate and octopamine individually are not essential for the sleep homeostat.

Third, I demonstrated that neuronal responsiveness is reduced following sleep deprivation. The stimulus evoked calcium transients of the mechanosensory neuron ALM are reduced following prolonged sleep deprivation compared to wake control. 
Chapter 4. Discussion 


\section{Bibliography}

[1] H. Pieron, "Le probleme physiologique du sommeil," Masson, 1913.

[2] S. S. Campbell and I. Tobler, "Animal sleep: a review of sleep duration across phylogeny.," Neurosci Biobehav Rev, vol. 8, no. 3, pp. 269-300, 1984.

[3] I. Tobler and A. A. Borbély, "Effect of rest deprivation on motor activity of fish.," J Comp Physiol A, vol. 157, no. 6, pp. 817-822, 1985.

[4] C. M. Jung, E. L. Melanson, E. J. Frydendall, L. Perreault, R. H. Eckel, and K. P. Wright, "Energy expenditure during sleep, sleep deprivation and sleep following sleep deprivation in adult humans.," J Physiol, vol. 589, no. Pt 1, pp. 235-244, 2011.

[5] J. Kong, P. N. Shepel, C. P. Holden, M. Mackiewicz, A. I. Pack, and J. D. Geiger, "Brain glycogen decreases with increased periods of wakefulness: implications for homeostatic drive to sleep.," J Neurosci, vol. 22, no. 13, pp. 5581-5587, 2002.

[6] S. Daan, B. M. Barnes, and A. M. Strijkstra, "Warming up for sleep? ground squirrels sleep during arousals from hibernation.," Neurosci Lett, vol. 128, no. 2, pp. 265-268, 1991.

[7] L. A. Toth and J. M. Krueger, "Effects of microbial challenge on sleep in rabbits.," FASEB J, vol. 3, no. 9, pp. 2062-2066, 1989.

[8] J. M. Krueger, "The role of cytokines in sleep regulation.," Curr Pharm Des, vol. 14, no. 32, pp. 3408-3416, 2008.

[9] C. Smith, "Sleep states and memory processes in humans: procedural versus declarative memory systems.," Sleep Med Rev, vol. 5, no. 6, pp. 491-506, 2001.

[10] E. M. Robertson, A. Pascual-Leone, and R. C. Miall, "Current concepts in procedural consolidation.," Nat Rev Neurosci, vol. 5, no. 7, pp. 576-582, 2004.

[11] L. Marshall, H. Helgadóttir, M. Mölle, and J. Born, “Boosting slow oscillations during sleep potentiates memory.," Nature, vol. 444, no. 7119, pp. 610-613, 2006.

[12] V. V. Vyazovskiy, C. Cirelli, M. Pfister-Genskow, U. Faraguna, and G. Tononi, "Molecular and electrophysiological evidence for net synaptic potentiation in wake and depression in sleep.," Nat Neurosci, vol. 11, no. 2, pp. 200-208, 2008.

[13] M. A. Wilson and B. L. McNaughton, "Reactivation of hippocampal ensemble memories during sleep.," Science, vol. 265, no. 5172, pp. 676-679, 1994. 
[14] S. Ribeiro, X. Shi, M. Engelhard, Y. Zhou, H. Zhang, D. Gervasoni, S.-C. Lin, K. Wada, N. A. M. Lemos, and M. A. L. Nicolelis, "Novel experience induces persistent sleep-dependent plasticity in the cortex but not in the hippocampus.," Front Neurosci, vol. 1, no. 1, pp. 43-55, 2007.

[15] S. Gais, G. Albouy, M. Boly, T. T. Dang-Vu, A. Darsaud, M. Desseilles, G. Rauchs, M. Schabus, V. Sterpenich, G. Vandewalle, P. Maquet, and P. Peigneux, "Sleep transforms the cerebral trace of declarative memories.," Proc Natl Acad Sci U S A, vol. 104, no. 47, pp. 18778-18783, 2007.

[16] L. Xie, H. Kang, Q. Xu, M. J. Chen, Y. Liao, M. Thiyagarajan, J. O'Donnell, D. J. Christensen, C. Nicholson, J. J. Iliff, T. Takano, R. Deane, and M. Nedergaard, "Sleep drives metabolite clearance from the adult brain.," Science, vol. 342, no. 6156, pp. 373-377, 2013.

[17] C. Cirelli and G. Tononi, "Is sleep essential?," PLoS Biol, vol. 6, no. 8, p. e216, 2008.

[18] R. E. Mistlberger and D. J. Skene, "Nonphotic entrainment in humans?," J Biol Rhythms, vol. 20, no. 4, pp. 339-352, 2005.

[19] A. Borbely, "Sleep: circadian rhythm versus recovery process," In: M.Koukkou, D.Lehmann and J.Angst (Eds) Functional States of the Brain: Their Determinants. Elsevier, Amsterdam, 1980.

[20] D. J. Dijk, D. G. Beersma, S. Daan, G. M. Bloem, and R. H. Van den Hoofdakker, "Quantitative analysis of the effects of slow wave sleep deprivation during the first $3 \mathrm{~h}$ of sleep on subsequent eeg power density.," Eur Arch Psychiatry Neurol Sci, vol. 236, no. 6, pp. 323-328, 1987.

[21] V. V. Vyazovskiy and I. Tobler, "Theta activity in the waking eeg is a marker of sleep propensity in the rat.," Brain Res, vol. 1050, no. 1-2, pp. 64-71, 2005.

[22] I. Tobler, A. A. Borbély, and G. Groos, "The effect of sleep deprivation on sleep in rats with suprachiasmatic lesions.," Neurosci Lett, vol. 42, no. 1, pp. 49-54, 1983.

[23] P. Franken and D.-J. Dijk, "Circadian clock genes and sleep homeostasis.," Eur J Neurosci, vol. 29, no. 9, pp. 1820-1829, 2009.

[24] J. E. Sherin, P. J. Shiromani, R. W. McCarley, and C. B. Saper, "Activation of ventrolateral preoptic neurons during sleep.," Science, vol. 271, no. 5246, pp. 216-219, 1996.

[25] R. Szymusiak, T. Steininger, N. Alam, and D. McGinty, "Preoptic area sleepregulating mechanisms.," Arch Ital Biol, vol. 139, no. 1-2, pp. 77-92, 2001.

[26] K. Takahashi, J.-S. Lin, and K. Sakai, "Characterization and mapping of sleepwaking specific neurons in the basal forebrain and preoptic hypothalamus in mice.," Neuroscience, vol. 161, no. 1, pp. 269-292, 2009. 
[27] J. E. Sherin, J. K. Elmquist, F. Torrealba, and C. B. Saper, "Innervation of histaminergic tuberomammillary neurons by gabaergic and galaninergic neurons in the ventrolateral preoptic nucleus of the rat.," J Neurosci, vol. 18, no. 12, pp. 4705$4721,1998$.

[28] J. H. Benington and H. C. Heller, "Restoration of brain energy metabolism as the function of sleep.," Prog Neurobiol, vol. 45, no. 4, pp. 347-360, 1995.

[29] T. Porkka-Heiskanen, R. E. Strecker, M. Thakkar, A. A. Bjorkum, R. W. Greene, and R. W. McCarley, "Adenosine: a mediator of the sleep-inducing effects of prolonged wakefulness.," Science, vol. 276, no. 5316, pp. 1265-1268, 1997.

[30] T. Gallopin, P. Fort, E. Eggermann, B. Cauli, P. H. Luppi, J. Rossier, E. Audinat, M. Mühlethaler, and M. Serafin, "Identification of sleep-promoting neurons in vitro.," Nature, vol. 404, no. 6781, pp. 992-995, 2000.

[31] A. V. Kalinchuk, R. W. McCarley, D. Stenberg, T. Porkka-Heiskanen, and R. Basheer, "The role of cholinergic basal forebrain neurons in adenosinemediated homeostatic control of sleep: lessons from 192 igg-saporin lesions.," Neuroscience, vol. 157, no. 1, pp. 238-253, 2008.

[32] U. Faraguna, V. V. Vyazovskiy, A. B. Nelson, G. Tononi, and C. Cirelli, "A causal role for brain-derived neurotrophic factor in the homeostatic regulation of sleep.," J Neurosci, vol. 28, no. 15, pp. 4088-4095, 2008.

[33] C. Cirelli, "The genetic and molecular regulation of sleep: from fruit flies to humans.," Nat Rev Neurosci, vol. 10, no. 8, pp. 549-560, 2009.

[34] R. J. Barstead and D. G. Moerman, “C. elegans deletion mutant screening.," Methods Mol Biol, vol. 351, pp. 51-58, 2006.

[35] S. Mitani, "Nematode, an experimental animal in the national bioresource project.," Exp Anim, vol. 58, no. 4, pp. 351-356, 2009.

[36] D. M. Raizen, J. E. Zimmerman, M. H. Maycock, U. D. Ta, Y.-j. You, M. V. Sundaram, and A. I. Pack, "Lethargus is a caenorhabditis elegans sleep-like state.," Nature, vol. 451, no. 7178, pp. 569-572, 2008.

[37] R. C. Cassada and R. L. Russell, "The dauerlarva, a post-embryonic developmental variant of the nematode caenorhabditis elegans.," Dev Biol, vol. 46, no. 2, pp. 326-342, 1975.

[38] S. Iwanir, N. Tramm, S. Nagy, C. Wright, D. Ish, and D. Biron, "The microarchitecture of c. elegans behavior during lethargus: homeostatic bout dynamics, a typical body posture, and regulation by a central neuron.," Sleep, vol. 36, no. 3, pp. 385-395, 2013. 
[39] J. Schwarz, I. Lewandrowski, and H. Bringmann, "Reduced activity of a sensory neuron during a sleep-like state in caenorhabditis elegans.," Curr Biol, vol. 21, no. 24, pp. R983-R984, 2011.

[40] M. Turek, I. Lewandrowski, and H. Bringmann, "An ap2 transcription factor is required for a sleep-active neuron to induce sleep-like quiescence in c. elegans.," Curr Biol, vol. 23, no. 22, pp. 2215-2223, 2013.

[41] R. J. Driver, A. L. Lamb, A. J. Wyner, and D. M. Raizen, "Daf-16/foxo regulates homeostasis of essential sleep-like behavior during larval transitions in c. elegans.," Curr Biol, vol. 23, no. 6, pp. 501-506, 2013.

[42] J. Schwarz, J.-P. Spies, and H. Bringmann, "Reduced muscle contraction and a relaxed posture during sleep-like lethargus.," Worm, vol. 1, no. 1, pp. 12-14, 2012.

[43] M. Jeon, H. F. Gardner, E. A. Miller, J. Deshler, and A. E. Rougvie, "Similarity of the c. elegans developmental timing protein lin-42 to circadian rhythm proteins.," Science, vol. 286, no. 5442, pp. 1141-1146, 1999.

[44] G. C. Monsalve, C. Van Buskirk, and A. R. Frand, "Lin-42/period controls cyclical and developmental progression of c. elegans molts.," Curr Biol, vol. 21, no. 24, pp. 2033-2045, 2011.

[45] T. Saigusa, S. Ishizaki, S. Watabiki, N. Ishii, A. Tanakadate, Y. Tamai, and K. Hasegawa, "Circadian behavioural rhythm in caenorhabditis elegans.," Curr Biol, vol. 12, no. 2, pp. R46-R47, 2002.

[46] M. L. Migliori, S. H. Simonetta, A. Romanowski, and D. A. Golombek, "Circadian rhythms in metabolic variables in caenorhabditis elegans.," Physiol Behav, vol. 103, no. 3-4, pp. 315-320, 2011.

[47] A. M. van der Linden, M. Beverly, S. Kadener, J. Rodriguez, S. Wasserman, M. Rosbash, and P. Sengupta, "Genome-wide analysis of light- and temperatureentrained circadian transcripts in caenorhabditis elegans.," PLoS Biol, vol. 8, no. 10, p. e1000503, 2010.

[48] K. Singh, J. Y. Ju, M. B. Walsh, M. A. Dilorio, and A. C. Hart, “Deep conservation of genes required for both drosphila melanogaster and caenorhabditis elegans sleep includes a role for dopaminergic signaling.," Sleep, vol. 37, no. 9, pp. 14391451, 2014.

[49] K. Singh, M. Y. Chao, G. A. Somers, H. Komatsu, M. E. Corkins, J. Larkins-Ford, T. Tucey, H. M. Dionne, M. B. Walsh, E. K. Beaumont, D. P. Hart, S. R. Lockery, and A. C. Hart, "C. elegans notch signaling regulates adult chemosensory response and larval molting quiescence.," Curr Biol, vol. 21, no. 10, pp. 825-834, 2011. 
[50] M. D. Nelson, K. H. Lee, M. A. Churgin, A. J. Hill, C. Van Buskirk, C. Fang-Yen, and D. M. Raizen, "Fmrfamide-like flp-13 neuropeptides promote quiescence following heat stress in caenorhabditis elegans.," Curr Biol, vol. 24, no. 20, pp. 24062410, 2014.

[51] M. D. Nelson, N. F. Trojanowski, J. B. George-Raizen, C. J. Smith, C.-C. Yu, C. Fang-Yen, and D. M. Raizen, "The neuropeptide nlp-22 regulates a sleep-like state in caenorhabditis elegans.," Nat Commun, vol. 4, p. 2846, 2013.

[52] J. E. Sulston and S. Brenner, "The dna of caenorhabditis elegans.," Genetics, vol. 77, no. 1, pp. 95-104, 1974.

[53] T. Stiernagle, “Maintenance of c. elegans.," WormBook, pp. 1-11, 2006.

[54] J. L. Hartley, G. F. Temple, and M. A. Brasch, "Dna cloning using in vitro sitespecific recombination.," Genome Res, vol. 10, no. 11, pp. 1788-1795, 2000.

[55] S. F. Altschul, W. Gish, W. Miller, E. W. Myers, and D. J. Lipman, “Basic local alignment search tool.," J Mol Biol, vol. 215, no. 3, pp. 403-410, 1990.

[56] D. T. Stinchcomb, J. E. Shaw, S. H. Carr, and D. Hirsh, "Extrachromosomal dna transformation of caenorhabditis elegans.," Mol Cell Biol, vol. 5, no. 12, pp. 34843496, 1985.

[57] T. Wilm, P. Demel, H. U. Koop, H. Schnabel, and R. Schnabel, "Ballistic transformation of caenorhabditis elegans.," Gene, vol. 229, no. 1-2, pp. 31-35, 1999.

[58] V. Praitis, E. Casey, D. Collar, and J. Austin, "Creation of low-copy integrated transgenic lines in caenorhabditis elegans.," Genetics, vol. 157, no. 3, pp. 12171226, 2001.

[59] J. Ahringer, "Reverse genetics.," Wormbook, 2000.

[60] R. J. Barstead, L. Kleiman, and R. H. Waterston, "Cloning, sequencing, and mapping of an alpha-actinin gene from the nematode caenorhabditis elegans.," Cell Motil Cytoskeleton, vol. 20, no. 1, pp. 69-78, 1991.

[61] T. A. Pologruto, R. Yasuda, and K. Svoboda, "Monitoring neural activity and [ca2+] with genetically encoded ca2+ indicators.," J Neurosci, vol. 24, no. 43, pp. 9572-9579, 2004.

[62] J. Nakai, M. Ohkura, and K. Imoto, "A high signal-to-noise ca(2+) probe composed of a single green fluorescent protein.," Nat Biotechnol, vol. 19, no. 2, pp. 137141, 2001.

[63] L. Tian, S. A. Hires, T. Mao, D. Huber, M. E. Chiappe, S. H. Chalasani, L. Petreanu, J. Akerboom, S. A. McKinney, E. R. Schreiter, C. I. Bargmann, V. Jayaraman, K. Svoboda, and L. L. Looger, "Imaging neural activity in worms, flies and mice 
with improved gcamp calcium indicators.," Nat Methods, vol. 6, no. 12, pp. 875881, 2009.

[64] H. Suzuki, R. Kerr, L. Bianchi, C. Frøkjaer-Jensen, D. Slone, J. Xue, B. Gerstbrein, M. Driscoll, and W. R. Schafer, "In vivo imaging of c. elegans mechanosensory neurons demonstrates a specific role for the mec- 4 channel in the process of gentle touch sensation.," Neuron, vol. 39, no. 6, pp. 1005-1017, 2003.

[65] D. Shcherbo, C. S. Murphy, G. V. Ermakova, E. A. Solovieva, T. V. Chepurnykh, A. S. Shcheglov, V. V. Verkhusha, V. Z. Pletnev, K. L. Hazelwood, P. M. Roche, S. Lukyanov, A. G. Zaraisky, M. W. Davidson, and D. M. Chudakov, "Far-red fluorescent tags for protein imaging in living tissues.," Biochem J, vol. 418, no. 3, pp. 567-574, 2009.

[66] S. Redemann, S. Schloissnig, S. Ernst, A. Pozniakowsky, S. Ayloo, A. A. Hyman, and $\mathrm{H}$. Bringmann, "Codon adaptation-based control of protein expression in c. elegans.," Nat Methods, vol. 8, no. 3, pp. 250-252, 2011.

[67] M. Hendricks, H. Ha, N. Maffey, and Y. Zhang, “Compartmentalized calcium dynamics in a c. elegans interneuron encode head movement.," Nature, vol. 487, no. 7405, pp. 99-103, 2012.

[68] E. S. Boyden, F. Zhang, E. Bamberg, G. Nagel, and K. Deisseroth, "Millisecondtimescale, genetically targeted optical control of neural activity.," Nat Neurosci, vol. 8, no. 9, pp. 1263-1268, 2005.

[69] G. Nagel, M. Brauner, J. F. Liewald, N. Adeishvili, E. Bamberg, and A. Gottschalk, "Light activation of channelrhodopsin-2 in excitable cells of caenorhabditis elegans triggers rapid behavioral responses.," Curr Biol, vol. 15, no. 24, pp. 2279$2284,2005$.

[70] M. Hegemann, P. Fuhrmann and S. Kateriya, "Algal sensory photoreceptors," J. Phycology, 2001.

[71] G. Nagel, T. Szellas, W. Huhn, S. Kateriya, N. Adeishvili, P. Berthold, D. Ollig, P. Hegemann, and E. Bamberg, "Channelrhodopsin-2, a directly light-gated cation-selective membrane channel.," Proc Natl Acad Sci U S A, vol. 100, no. 24, pp. 13940-13945, 2003.

[72] N. C. Klapoetke, Y. Murata, S. S. Kim, S. R. Pulver, A. Birdsey-Benson, Y. K. Cho, T. K. Morimoto, A. S. Chuong, E. J. Carpenter, Z. Tian, J. Wang, Y. Xie, Z. Yan, Y. Zhang, B. Y. Chow, B. Surek, M. Melkonian, V. Jayaraman, M. ConstantinePaton, G. K.-S. Wong, and E. S. Boyden, "Independent optical excitation of distinct neural populations.," Nat Methods, vol. 11, no. 3, pp. 338-346, 2014.

[73] J. Y. Lin, P. M. Knutsen, A. Muller, D. Kleinfeld, and R. Y. Tsien, “Reachr: a redshifted variant of channelrhodopsin enables deep transcranial optogenetic excitation.," Nat Neurosci, vol. 16, no. 10, pp. 1499-1508, 2013. 
[74] X. Han and E. S. Boyden, "Multiple-color optical activation, silencing, and desynchronization of neural activity, with single-spike temporal resolution.," PLoS One, vol. 2, no. 3, p. e299, 2007.

[75] B. Y. Chow, X. Han, A. S. Dobry, X. Qian, A. S. Chuong, M. Li, M. A. Henninger, G. M. Belfort, Y. Lin, P. E. Monahan, and E. S. Boyden, "High-performance genetically targetable optical neural silencing by light-driven proton pumps.," Nature, vol. 463, no. 7277, pp. 98-102, 2010.

[76] H. Bringmann, "Agarose hydrogel microcompartments for imaging sleep- and wake-like behavior and nervous system development in caenorhabditis elegans larvae.," J Neurosci Methods, vol. 201, no. 1, pp. 78-88, 2011.

[77] M. Chalfie, J. E. Sulston, J. G. White, E. Southgate, J. N. Thomson, and S. Brenner, "The neural circuit for touch sensitivity in caenorhabditis elegans.," J Neurosci, vol. 5, no. 4, pp. 956-964, 1985.

[78] R. A. Cabeza, R. Liese, A. Lingner, I. von Stieglitz, J. Neumann, G. Salinas-Riester, C. Pommerenke, K. Dittert, and J. Schulze, "Rna-seq transcriptome profiling reveals that medicago truncatula nodules acclimate $n$ ? fixation before emerging $p$ deficiency reaches the nodules.," J Exp Bot, vol. 65, no. 20, pp. 6035-6048, 2014.

[79] M. Dodt, J. T. Roehr, R. Ahmed, and C. Dieterich, "Flexbarflexible barcode and adapter processing for next-generation sequencing platforms," Biology, vol. 1, no. 3, pp. 895-905, 2012.

[80] T. Wang, J. Liu, L. Shen, J. Tonti-Filippini, Y. Zhu, H. Jia, R. Lister, J. Ecker, A. H. Millar, B. Ren, et al., "Star: an integrated solution to management and visualization of sequencing data," Bioinformatics, vol. 29, no. 24, pp. 3204-3210, 2013.

[81] S. Anders and W. Huber, "Differential expression analysis for sequence count data," Genome biol, vol. 11, no. 10, p. R106, 2010.

[82] Y. Benjamini and Y. Hochberg, "Controlling the false discovery rate: a practical and powerful approach to multiple testing," Journal of the Royal Statistical Society. Series B (Methodological), pp. 289-300, 1995.

[83] S. J. Husson, W. S. Costa, C. Schmitt, and A. Gottschalk, "Keeping track of worm trackers.," WormBook, pp. 1-17, 2012.

[84] R. Ghosh and S. W. Emmons, "Episodic swimming behavior in the nematode c. elegans.," J Exp Biol, vol. 211, no. Pt 23, pp. 3703-3711, 2008.

[85] S. L. Edwards, N. K. Charlie, M. C. Milfort, B. S. Brown, C. N. Gravlin, J. E. Knecht, and K. G. Miller, "A novel molecular solution for ultraviolet light detection in caenorhabditis elegans.," PLoS Biol, vol. 6, no. 8, p. e198, 2008. 
[86] O. Yizhar, L. E. Fenno, M. Prigge, F. Schneider, T. J. Davidson, D. J. O'Shea, V. S. Sohal, I. Goshen, J. Finkelstein, J. T. Paz, K. Stehfest, R. Fudim, C. Ramakrishnan, J. R. Huguenard, P. Hegemann, and K. Deisseroth, "Neocortical excitation/inhibition balance in information processing and social dysfunction.," $\mathrm{Na}$ ture, vol. 477, no. 7363, pp. 171-178, 2011.

[87] G. F. Buchanan and G. B. Richerson, "Central serotonin neurons are required for arousal to co2.," Proc Natl Acad Sci U S A, vol. 107, no. 37, pp. 16354-16359, 2010.

[88] O. Aurelio, T. Boulin, and O. Hobert, "Identification of spatial and temporal cues that regulate postembryonic expression of axon maintenance factors in the c. elegans ventral nerve cord.," Development, vol. 130, no. 3, pp. 599-610, 2003.

[89] O. Hobert, K. Tessmar, and G. Ruvkun, "The caenorhabditis elegans lim-6 lim homeobox gene regulates neurite outgrowth and function of particular gabaergic neurons.," Development, vol. 126, no. 7, pp. 1547-1562, 1999.

[90] E. L. Tsalik, T. Niacaris, A. S. Wenick, K. Pau, L. Avery, and O. Hobert, "Lim homeobox gene-dependent expression of biogenic amine receptors in restricted regions of the c. elegans nervous system.," Dev Biol, vol. 263, no. 1, pp. 81-102, 2003.

[91] A. Antebi, "Nuclear hormone receptors in c. elegans.," WormBook, pp. 1-13, 2006.

[92] S. Sarin, C. Antonio, B. Tursun, and O. Hobert, "The c. elegans tailless/tlx transcription factor nhr-67 controls neuronal identity and left/right asymmetric fate diversification.," Development, vol. 136, no. 17, pp. 2933-2944, 2009.

[93] D. A. Glauser, W. C. Chen, R. Agin, B. L. Macinnis, A. B. Hellman, P. A. Garrity, M.-W. Tan, and M. B. Goodman, "Heat avoidance is regulated by transient receptor potential (trp) channels and a neuropeptide signaling pathway in caenorhabditis elegans.," Genetics, vol. 188, no. 1, pp. 91-103, 2011.

[94] J. Takayama, S. Faumont, H. Kunitomo, S. R. Lockery, and Y. Iino, "Single-cell transcriptional analysis of taste sensory neuron pair in caenorhabditis elegans.," Nucleic Acids Res, vol. 38, no. 1, pp. 131-142, 2010.

[95] C. Li and K. Kim, “Neuropeptides.,” WormBook, pp. 1-36, 2008.

[96] J. Kass, T. C. Jacob, P. Kim, and J. M. Kaplan, "The egl-3 proprotein convertase regulates mechanosensory responses of caenorhabditis elegans.," J Neurosci, vol. 21, no. 23, pp. 9265-9272, 2001.

[97] V. Lainé, C. Frøkjær-Jensen, H. Couchoux, and M. Jospin, "The alpha1 subunit egl-19, the alpha2/delta subunit unc-36, and the beta subunit ccb-1 underlie voltage-dependent calcium currents in caenorhabditis elegans striated muscle.," J Biol Chem, vol. 286, no. 42, pp. 36180-36187, 2011. 
[98] Z. Altun and D. Hall, "Wormatlas," Altun ZF, Herndon LA, Crocker C, Lints R, Hall DH, eds, 2002.

[99] J. E. Sulston and H. R. Horvitz, "Post-embryonic cell lineages of the nematode, caenorhabditis elegans.," Dev Biol, vol. 56, no. 1, pp. 110-156, 1977.

[100] S. Nagy, D. M. Raizen, and D. Biron, "Measurements of behavioral quiescence in caenorhabditis elegans.," Methods, vol. 68, no. 3, pp. 500-507, 2014.

[101] I. V. Zhdanova, "Sleep in zebrafish.," Zebrafish, vol. 3, no. 2, pp. 215-226, 2006.

[102] C. B. Saper, T. E. Scammell, and J. Lu, "Hypothalamic regulation of sleep and circadian rhythms.," Nature, vol. 437, no. 7063, pp. 1257-1263, 2005.

[103] B. L. Chen, D. H. Hall, and D. B. Chklovskii, "Wiring optimization can relate neuronal structure and function.," Proc Natl Acad Sci U S A, vol. 103, no. 12, pp. 4723$4728,2006$.

[104] J. M. Donlea, D. Pimentel, and G. Miesenböck, "Neuronal machinery of sleep homeostasis in drosophila.," Neuron, vol. 81, no. 4, pp. 860-872, 2014.

[105] B. Zhao and W. R. Schafer, "Neuropeptide signaling: from the gut.," Curr Biol, vol. 23, no. 11, pp. R481-R483, 2013. 


\section{Acknowledgements}

First and foremost, I would like to thank my supervisor Henrik Bringmann for his continuous support and the great working conditions. He has given me the freedom to pursue my own scientific ideas and develop my research skills. I am grateful to Andreas Stumpner and Jens Frahm for their support as members of my thesis committee, and to Halyna Shcherbata, Ralf Heinrich, and Reinhard Schuh for participation in my extended committee.

I would like to thank also the Göttingen Graduate School for Neurosciences, Biophysics, and Molecular Biosciences for offering great method and soft-skill courses, administrative support and travel grants.

I would like to thank all members of the Sleep and Waking group for the good working atmosphere, fruitful discussions and not to be forgotten our great Kicker playing. I would especially like to thank Michał Turek for countless scientific discussions. I also thank Ines Lewandrowski and Juliane Schwarz for introducing me to molecular biology and genetics.

I thank Boris Busche and Jan-Martin Kirves of the 3D Electron Cryo-Microscopy group and Birk Urmersbach for their support in the devolopment of $C$. elegans nose tracking software. I want to thank our Precision Mechanics Workshop and IT \& Electronics service for their excellent custom-built devices and their continuous and quick support. I especially thank Hans-Dieter Scheede for building the dish tapper and electronic devices to trigger the sleep deprivation stimulus.

I want to thank Ratna Wolfram and Michael Feyerabend for their assistance performing C. elegans crossings. I thank Julia Borstel, Alexandra Stützer, Louisa Welp, Cornelia Roth and Michael Pilot for their help with manual data processing. I would like to thank the Caenorhabditis Genetics Center, the Mitani Laboratory at the TokyoWomen's University and the laboratories of Bill Schafer, Oliver Hobert, Alexander Gottschalk, Elias Peles and Yun Zhang for sharing C. elegans strains. Not to forget the work of Silvia Gremmler and Michael Pilot, preparing media and cleaning glassware, as this daily support was invaluable help. Also I want to show my appreciation for Franziska Schmidt's administrative support. I thank Michał Turek, Judith Frontzeck, Florentin Masurat, Judith Besseling, Seychelle Vos, Maren Spies and Maria Tauber for proofreading and suggestions.

Finally, I thank my great family and friends for their continuous support. 



\section{Appendix}

\section{A Abbreviations and glossary}

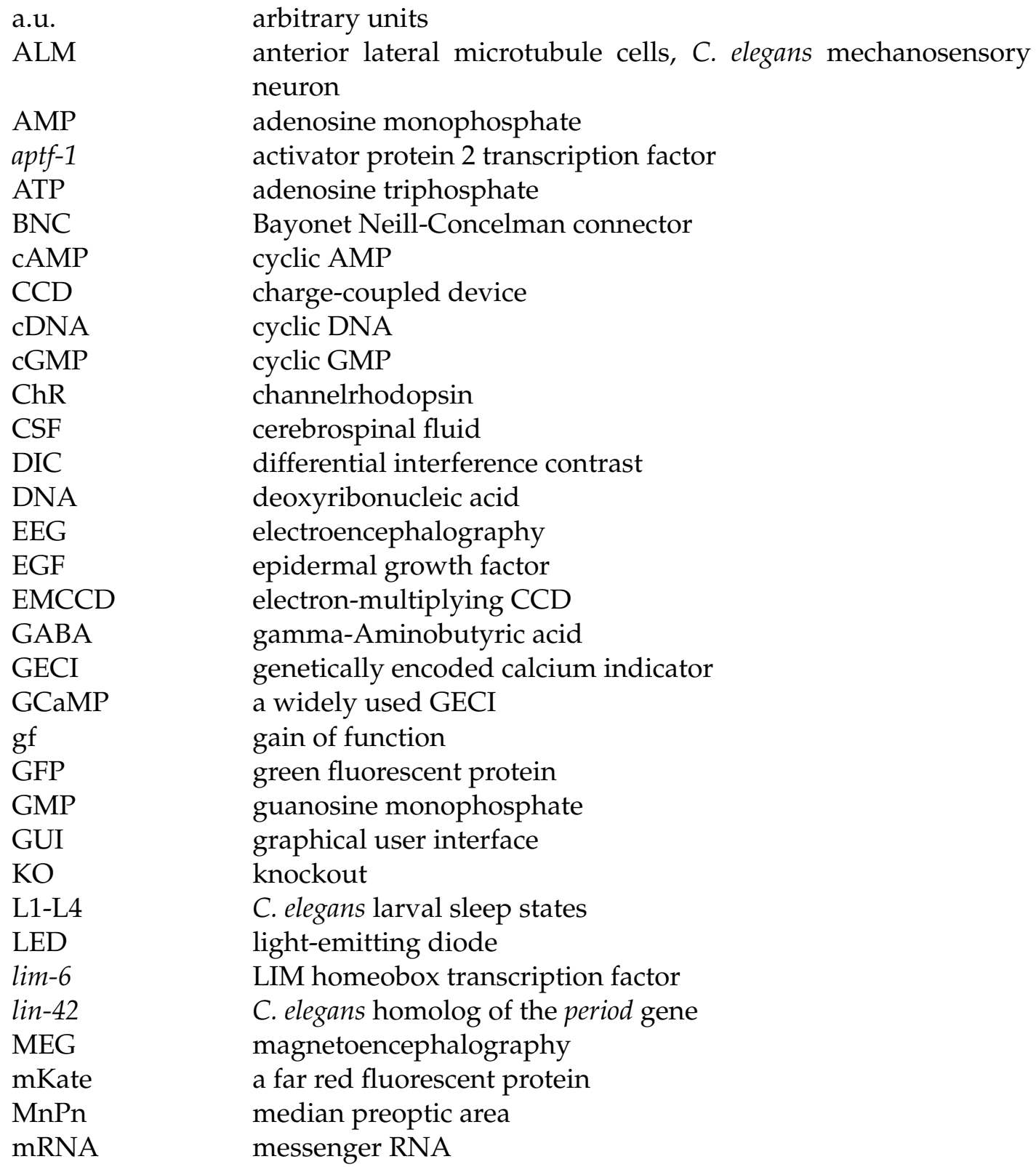




$\begin{array}{ll}\text { NGM } & \text { nematode growth medium } \\ \text { NP } & \text { non pumping period } \\ \text { NREM } & \text { non-rapid eye movement } \\ \text { PCR } & \text { polymerase chain reaction } \\ \text { PDMS } & \text { polydimethylsiloxane } \\ \text { PLM } & \text { posterior lateral microtubule cells, C. elegans mechanosensory } \\ & \text { neuron } \\ \text { REM } & \text { rapid eye movement } \\ \text { RIS } & \text { C. elegans interneuron } \\ \text { RNA } & \text { ribonucleic acid } \\ \text { RNAi } & \text { RNA interference } \\ \text { sCMOS } & \text { complementary metal-oxide-semiconductor } \\ \text { SCN } & \text { suprachiasmatic nucleus } \\ \text { SD } & \text { sleep deprivation } \\ \text { Std } & \text { standard deviation } \\ \text { SWS } & \text { slow wave activity } \\ \text { TES } & \text { transcranial electrical stimulation } \\ \text { TTL } & \text { transistor-transistor logic } \\ \text { TNF } & \text { tumor necrosis factor } \\ \text { VI } & \text { virtual instrument } \\ \text { VLPO } & \text { ventrolateral preoptic nucleus }\end{array}$




\section{B C. elegans strains, constructs and primers used/generated during this thesis}

Most strains used during this thesis that were not generated in our laboratory were ordered from the Caenorhabditis Genetics Center (CGC) which is funded by NIH Office of Research Infrastructure Programs (P40 OD010440). tm alleles were provided by the Mitani Laboratory at the TokyoWomen's Medical University School of Medicine.

\section{B.1 C.elegans strain list}

Strains that I generated are marked by an asterisk $\left(^{*}\right)$. Strains that have been used for experiments described in this thesis are marked by the capital letter E (E).

\footnotetext{
HBR1 goeIs1[punc-119::SL1-GCamP3.35-SL2::unc-54-3'utr, unc-119(+)]. (E)

HBR4 goeIs3[pmyo::GCamP3.35::unc-54-3'utr, unc-119(+)]. (E)

HBR190 goeIs28[pnmr-1::Chr2::mKate2-unc-54-3'utr, unc-119(+)]. (E)

HBR205 goeIs22[pmec-4::SL1-GCaMP3.35-SL2:::mKate2-unc-54-3'utr, unc-119(+)]. (E)
HBR222 goeIs43[pmec-4::Chr2::mKate2-unc-54-3'utr, unc-119(+)]. (E)

HBR432 unc-119(ed3), goeEx250[punc-119::C1V1::mKate2-unc54-3'utr, unc-119(+)].

$(*)$

HBR433 unc-119(ed3), goeEx251[punc-119::C1V1::mKate2-unc54-3'utr, unc-119(+)].

$(*)$

HBR434 unc-119(ed3), goeEx252[punc-119::C1V1::mKate2-unc54-3'utr, unc-119(+)].

$(*)$

HBR510 goeIs1[punc-119::SL1-GCamP3.35-SL2::unc-54-3'utr line 3-35, unc-119(+)], ljIS105[psra-6::ChR2::yfp, unc-122::gfp]. $\left({ }^{*}, \mathrm{E}\right)$

HBR511 him-5(e1490), goeIs22[pmec-4::SL1-GCaMP3.35-SL2::mKate2-unc-54-3'utr, unc-119(+)], syIs197. $\left({ }^{*}\right)$

HBR513 egl-4(ad450), goeIs22[pmec-4::SL1-GCaMP3.35-SL2::mKate2-unc-54-3'utr, unc-119(+)]. $\left(^{*}\right)$

HBR543 unc-119(ed3), goeIs118[aptf-1-5'utr::SL1-GCaMP3.35-SL2::mKate2-aptf-13'utr, unc-119(+)]. (*)

HBR556 aptf-1(gk794), goeIs118[aptf-1-5'utr::SL1-GCaMP3.35-SL2::mKate2-aptf-13'utr, unc-119(+)]. (E)

HBR606 lim-6(nr2073), goeIs118[aptf-1-5'utr::SL1-GCaMP3.35-SL2::mKate2-aptf-13'utr, unc-119(+)]. $\left.{ }^{*}, \mathrm{E}\right)$

HBR607 ser-4(ok512), goeIs118[aptf-1-5'utr::SL1-GCaMP3.35-SL2::mKate2-aptf-13'utr, unc-119(+)]. $\left.{ }^{*}, \mathrm{E}\right)$

HBR608 dop-1(vs100), goeIs118[aptf-1-5'utr::SL1-GCaMP3.35-SL2::mKate2-aptf-13'utr, unc-119(+)]. $\left({ }^{*}, \mathrm{E}\right)$

HBR609 ser-4(ok512), goeIs102[aptf-1-5'utr::ChR2::mKate2-aptf-1-3'utr, unc-119(+)]. $(*, \mathrm{E})$
} 
Bibliography

HBR610 dop-1(vs100), goels102[aptf-1-5'utr::ChR2::mKate2-aptf-1-3'utr, unc-119(+)]. $(*, \mathrm{E})$

HBR611 flp-11(tm2706), goeIs72[aptf-1-5'utr::d1mgfp::aptf-1-3'utr, unc-119(+)], goeIs22[pmec-4::SL1-GCaMP3.35-SL2::mKate2-unc-54-3'utr, unc-119(+)].

$(*)$

HBR612 goeIs22[pmec-4::SL1-GCaMP3.35-SL2::mKate2-unc-54-3'utr, unc-119(+)], lite-1(ce314), ljIs114[pgpa-1::FLPase, Psra-6::FTF::ChR2::YFP]. $\left(^{*}\right)$

HBR666 goeIs22[pmec-4::SL1-GCaMP3.35-SL2::mKate2-unc-54-3'utr, unc-119(+)], goeIs118[aptf-1-5'utr::SL1-GCaMP3.35-SL2::mKate2-aptf-1-3'utr, unc$119(+)] .(*, \mathrm{E})$

HBR669 ser-4(ok512), dop-1(vs100), goeIs118[aptf-1-5'utr::SL1-GCaMP3.35SL2::mKate2-aptf-1-3'utr, unc-119(+)]. $\left.{ }^{*}, \mathrm{E}\right)$

HBR676 egl-19(n2368), goeIs118[aptf-1-5'utr::SL1-GCaMP3.35-SL2::mKate2-aptf-13'utr, unc-119(+)]. (E)

HBR682 goeIs1[punc-119::SL1-GCaMP3.35-SL2::unc-54-3'utr, unc-119(+)], goeIs102[aptf-1-5'utr::ChR2::mKate2-aptf-1-3'utr, unc-119(+)]. $\left.{ }^{*}\right)$

HBR683 lim-6(nr2073), goeIs102[aptf-1-5'utr::ChR2::mKate2-aptf-1-3'utr, unc-119(+)]. $(*, E)$

HBR684 bas-1(tm351), goeIs118[aptf-1-5'utr::SL1-GCaMP3.35-SL2::mKate2-aptf-1$3^{\prime} u t r$, unc-119(+)]. $\left({ }^{*}, \mathrm{E}\right)$

HBR685 tph-1(mg280), goeIs118[aptf-1-5'utr::SL1-GCaMP3.35-SL2::mKate2-aptf-13'utr, unc-119(+)]. $\left(^{*}, \mathrm{E}\right)$

HBR686 unc-25(e156), goeIs118[aptf-1-5'utr::SL1-GCaMP3.35-SL2::mKate2-aptf-13'utr, unc-119(+)]. $\left({ }^{*}, \mathrm{E}\right)$

HBR687 tph-1(mg280), goeIs167[aptf-1-5'utr::archT::mKate2-aptf-1-3'utr, unc-119(+)]. $(*)$

HBR737 zig-5(ok1065), goeIs118[aptf-1-5'utr::SL1-GCaMP3.35-SL2::mKate2-aptf-13'utr, unc-119(+)]. (*)

HBR738 elt-4(ca16), goeIs118[aptf-1-5'utr::SL1-GCaMP3.35-SL2::mKate2-aptf-1-3'utr, unc-119(+)]. $\left(^{*}\right)$

HBR756 unc-119(ed3), goeEx303[pnmr-1::Chrimson::mkate2-unc-54-3'utr, unc-119(+)], [pnmr-1::SL1-GCaMP3.35-SL2::unc-54-3'utr, unc-119(+)]. (E)

HBR780 unc-119(ed3), goeEx315[psra-6::Chrimsonbird::mkate2-unc-54-3'utr, unc119(+)]. (E)

HBR795 unc-119(ed3), goeIs176[punc-47minus193prom::lim6ab::mkate2-unc-54-3'utr, unc-119(+)]. $\left(^{*}\right)$

HBR796 unc-119(ed3), goeEx319[phsp-16.2::Iim6ab::mkate2-unc-54-3'utr, unc-119(+)].

$(*)$

HBR797 unc-119(ed3), goeEx320[phsp-16.2::lim6ab::mkate2-unc-54-3'utr, unc-119(+)].

$(*)$

HBR798 unc-119(ed3), goeEx321[phsp-16.2::lim6ab::mkate2-unc-54-3'utr, unc-119(+)].

$(*)$ 
HBR799 unc-119(ed3), goeEx322[phsp-16.2::Iim6ab::mkate2-unc-54-3'utr, unc-119(+)]. $(*)$

HBR800 unc-119(ed3), goeEx323[phsp-16.2::Iim6ab::mkate2-unc-54-3'utr, unc-119(+)]. $(*)$

HBR801 unc-119(ed3), goeIs177[plim-6-int4::Lim6ab::mkate2-unc-54-3'utr, unc-119(+)]. $(*)$

HBR802 unc-119(ed3), goeIs178[plim-6-int4::lim6ab::mkate2-unc-54-3'utr, unc-119(+)]. $(*)$

HBR803 unc-119(ed3), goeEx324[plim-6-int4::Iim6ab::mkate2-unc-54-3'utr, unc$\left.119(+)] .{ }^{*}\right)$

HBR804 unc-119(ed3), goeEx325[plim-6-int4::Iim6ab::mkate2-unc-54-3'utr, unc$119(+)] .\left(^{*}\right)$

HBR805 unc-119(ed3), goeEx326[plim-6-int4::ChR2::mkate2-unc-54-3'utr, unc-119(+)]. $\left({ }^{*}\right)$

HBR806 unc-119(ed3), goeEx327[plim-6-int4::GCaMP-3.35::mkate2-unc-54-3'utr, unc$119(+)] .\left(^{*}\right)$

HBR807 unc-119(ed3), goeIs179[punc-119::GCaMP-6s:::mkate2-unc-54-3'utr, unc$119(+)] .\left({ }^{*}\right)$

HBR808 lim-6(nr2073), goeIs24[punc-119::SL1-GCaMP3.35-SL2::mkate2-unc-54-3'utr, unc-119(+)]. $(*)$

HBR809 lim-6(nr2073), goeEx327[plim-6-int4::GCaMP-3.35::mkate2-unc-54-3'utr, unc$119(+)] .\left(^{*}\right)$

HBR810 lim-6(nr2073), goeEx326[plim-6-int4::ChR2::mkate2-unc-54-3'utr, unc$119(+)] .(*, \mathrm{E})$

HBR811 lim-6(nr2073), aptf-1(gk794) II, goeIs118[aptf-1-5'utr::SL1-GCaMP3.35SL2::mkate2-aptf-1-3'utr, unc-119(+)]. $\left.{ }^{*}, \mathrm{E}\right)$

HBR812 lim-6(nr2073), goeIs167[aptf-1-5'utr::archT::mkate2-aptf-1-3'utr, unc-119(+)].

$(*)$

HBR813 lim-6(nr2073), goeEx324[plim-6-int4::lim6ab::mkate2-unc-54-3'utr, unc$119(+)] .\left({ }^{*}, \mathrm{E}\right)$

HBR814 lim-6(nr2073), goeEx328[plim-6-int4::Iim6ab::mkate2-unc-54-3'utr, unc$119(+)] .\left({ }^{*}, \mathrm{E}\right)$

HBR815 lim-6(nr2073), goeIs177[plim-6-int4::Iim6ab::mkate2-unc-54-3'utr, unc$119(+)] .\left({ }^{*}\right)$

HBR816 lim-6(nr2073), goeEx323[plim-6-int4::lim6ab::mkate2-unc-54-3'utr, unc$119(+)] .\left({ }^{*}\right)$

HBR817 lim-6(nr2073), goeIs176[punc-47minus193prom::lim6ab::mkate2-unc-54-3'utr, unc-119(+)]. $\left({ }^{*}, \mathrm{E}\right)$

HBR818 unc-119(ed3), goeEx328[plim-6-int4::Lim6ab::mkate2-unc-54-3'utr, unc$119(+)] .\left({ }^{*}\right)$

HBR893 lim-6(nr2073), goeIs118[aptf-1-5'utr::SL1-GCaMP3.35-SL2::mKate2-aptf-13'utr, unc-119(+)]. $\left({ }^{*}, \mathrm{E}\right)$ 
Bibliography

HBR894 lim-6(nr2073), unc-119(ed3) III, goeIs201[paptf-1::GCaMP6s::mKate-aptf-13'utr, unc-119(+)]. (*)

HBR895 mzmEx199[punc-31::NLSGCaMP5K, punc-122::gfp], goeIs102[aptf-15'utr::ChR2::mKate2-aptf-1-3'utr, unc-119(+)]. $\left({ }^{*}, \mathrm{E}\right)$

HBR896 lim-6(nr2073), goeIs118[aptf-1-5'utr::SL1-GCaMP3.35-SL2::mKate2-aptf-13'utr, unc-119(+)]. $\left(^{*}, \mathrm{E}\right)$

HBR897 goeIs24[punc-119::SL1-GCaMP3.35-SL2::mKate2-unc-54-3'utr, unc-119(+)], goeIs102[aptf-1-5'utr::ChR2::mKate2-aptf-1-3'utr, unc-119(+)]. (*)

HBR900 itr-1(sa73), goeIs118[aptf-1-5'utr::SL1-GCaMP3.35-SL2::mKate2-aptf-1-3'utr, unc-119(+)]. (E)

HBR901 unc-36(e251), goeIs118[aptf-1-5'utr::SL1-GCaMP3.35-SL2::mKate2-aptf-13'utr, unc-119(+)]. (E)

HBR914 lim-6(tm4836). bc6-7x. $\left({ }^{*}, \mathrm{E}\right)$

HBR971 goeIs102[aptf-1-5'utr::ChR2::mKate2-aptf-1-3'utr, unc-119(+)], mzmEx199[punc-31::NLSGCaMP5K, punc-122::gfp]. $\left({ }^{*}, \mathrm{E}\right)$

HBR972 goeIs167[aptf-1-5'utr::archT::mKate2-aptf-1-3'utr, unc-119(+)], mzmEx199[punc-31::NLSGCaMP5K, punc-122::gfp]. (*)

HBR973 IjIs133[unc-47::GCaMP3-SL2-tagRFP-T], lim-6(tm4836). $\left({ }^{*}, \mathrm{E}\right)$

HBR1028 goeIs241[plim6-int4:lim6b:mkate2-unc-54-3'utr, unc-119(+)], unc-119(ed3). ( $\left.{ }^{*}\right)$ HBR1029 goeIs242[plim6-int4:lim6b:mkate2-unc-54-3'utr, unc-119(+)], unc-119(ed3). (*) HBR1030 goeIs243[plim6-int4:lim6b:mkate2-unc-54-3'utr, unc-119(+)], unc-119(ed3). (*) HBR1031 lim-6(tm4836), [plim6-int4:lim6b:mkate2-unc-54-3'utr, unc-119(+)], unc119(ed3). $\left({ }^{*}, \mathrm{E}\right)$

HBR1032 lim-6(tm4836), [plim6-int4:lim6b:mkate2-unc-54-3'utr, unc-119(+)], unc119(ed3). $\left({ }^{*}, \mathrm{E}\right)$

HBR1033 lim-6(tm4836), [plim6-int4:lim6b:mkate2-unc-54-3'utr, unc-119(+)], unc119(ed3). $\left({ }^{*}\right)$

HBR1069 unc-119(ed3), goeEx299[unc47nu486prom::GCaMP6s::mkate2-unc-54-3'utr, unc-119(+)], lim-6(tm4836). $\left({ }^{*}, \mathrm{E}\right)$

HBR1070 unc-119(ed3), goeEx301[unc47nu486prom::GCaMP6s::mkate2-unc-54-3'utr, unc-119(+)], lim-6(tm4836). $\left(^{*}\right)$

HBR1071 unc-119(ed3), goeEx327[plim-6-int4::GCaMP-3.35::mkate2-unc-54-3'utr, unc119(+)], lim-6(tm4836). (*)

HBR1072 otIs157, lim-6(nr2073). $\left({ }^{*}, \mathrm{E}\right)$

HBR1073 unc-119(ed3), goeEx383[lim6-int4:: archT::mkate2-unc-54-3'utr, unc-119(+)]. $(*, E)$

HBR1074 unc-119(ed3), goeEx383[lim6-int4:: archT::mkate2-unc-54-3'utr, unc-119(+)], lim-6(tm4836). $\left({ }^{*}, \mathrm{E}\right)$

HBR10XX egl-3(gk238), goeIs113[aptf-1-5'utr::SL1-GCaMP3.35-SL2::mKate2-aptf-1$3^{\prime}$ utr, unc-119(+)]. $\left({ }^{*}, \mathrm{E}\right)$

AQ2026 ljls105[psra-6::chr-2::yfp;unc-122::gfp]. (E)

AQ2967 IjIs133[unc-47::GCaMP3-SL2-tagRFP-T]. (E)

CB1112 cat-2(e1112). (E) 


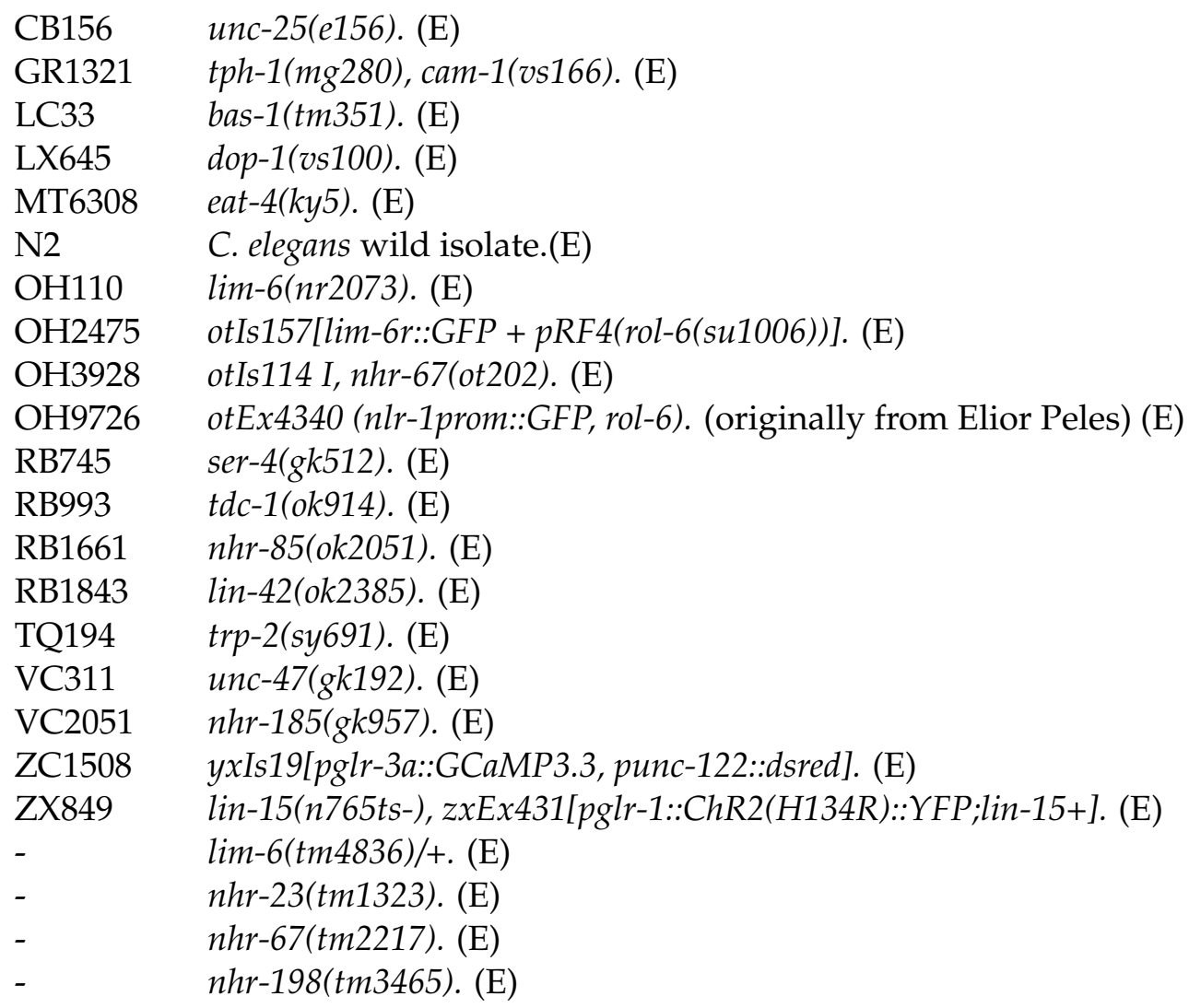

\section{B.2 Construct list}

Constructs that I cloned using the Multisite Gateway ${ }^{\mathrm{TM}}$ system from Invitrogen [54].

K59 pEntrL4-R1 punc-119 pEntrL1-L2 C1V1 "Katie" mKate2 unc-54 3'utr pCG150 unc-119 rescue fragment in $p D E S T R 4 R 3$

K60 pEntrL4-R1 pnmr-1 (pIR11) pEntrL1-L2 C1V1 "Katie" mKate2 unc-54 3'utr pCG150 unc-119 rescue fragment in $p D E S T R 4 R 3$

K79 pEntrL4-R1 pmec-4 pEntrL1-L2 C1V1 pEntrR2L3 unc-54 3'utr pCG150 unc119 rescue fragment in $p D E S T R 4 R 3$

K123 lim6int4 lim6ab pEntrR2L3 unc-54 3'utr pCG150 unc-119 rescue fragment in pDESTR $4 R 3$

K124 lim6int4 pEntrL1-L2 SL1-GCamP3.35-SL2 "Katie" mKate2 unc-54 3'utr pCG150 unc-119 rescue fragment in pDESTR4R3

K125 lim6int4 Chrimsonbird "Katie" mKate2 unc-54 3'utr pCG150 unc-119 rescue fragment in pDESTR4R3

K126 unc47minus193prom lim6ab pEntrR2L3 unc-54 3'utr pCG150 unc-119 rescue fragment in $p D E S T R 4 R 3$

K143 lim6int4 ChR2RCaMP1h34 pEntrR2L3 unc-54 3'utr pCG150 unc-119 rescue fragment in $p D E S T R 4 R 3$ 
K144 phsp16.2 lim6ab SL2mKate2unc-54-3UTR pCG150 unc-119 rescue fragment in pDESTR4R3

K145 pEntrL4-R1 punc-119 GCaMP6s "Katie" mKate2 unc-54 3'utr pCG150 unc119 rescue fragment in $p D E S T R 4 R 3$

K146 lim6int4 lim6ab SL2mKate2unc-54-3UTR pCG150 unc-119 rescue fragment in pDESTR4R3

K147 unc47minus193prom lim6ab SL2mKate2unc-54-3UTR pCG150 unc-119 rescue fragment in $p D E S T R 4 R 3$

K159 pEntrL4-R1 paptf-1 lim6ab pEntrR2L3 aptf-1 3'utr pCG150 unc-119 rescue fragment in $P D E S T R 4 R 3$

K160 unc47nu486prom lim6ab SL2mKate2unc-54-3UTR pCG150 unc-119 rescue fragment in $p D E S T R 4 R 3$

K161 pEntrL4-R1 paptf-1 lim6bSL2 mKate2 aptf-1-3'utr pCG150 unc-119 rescue fragment in $P D E S T R 4 R 3$

K162 unc47nu486prom lim6bSL2 "Katie" mKate2 unc-54 3'utr pCG150 unc-119 rescue fragment in $\mathrm{pDESTR} 4 \mathrm{R} 3$

\section{B.3 Primer list}

Primers that I created for duplex PCR genotyping of crossings.

lim-6(nr2073)

145 lim-6(nr2073)-fwd TCAGTCCCGGATAGCTGAAC

146 lim-6(nr2073)-rev CACAGGATAATTTGGTAGGAGCTT

147 N2-lim-6(nr2073)-fwd TAAAGCGGGTGGTTTGATTT

wt:229 mt: 322

lim-6(tm4836)

269 lim-6(tm4836)-fwd AACCCTCTCTCGGAACAGTG

270 lim-6(tm4836)-rev GGCTGAACAAGAAATCCGCA

271 N2-lim-6(tm4836)-rev AGACACCCACAACCAGAACA

wt: $290 \mathrm{mt}: 433$

dop-1(lx645)

156 dop-1(1x645)-fwd ACGCAAACTCTTCTGCCAAT

157 dop-1(lx645)-rev AGATTCAGGCGAGTTGCATT

158 N2-dop-1(1x645)-fwd GCTCAAACATCAGCGATCAG

$\mathrm{wt}: 281 \mathrm{mt}: 401$

ser-4(ok512) 
153 ser-4(ok512)-fwd AATCGGCCGAGTTATGACG 154 ser-4(ok512)-rev ATGGAACGGAGCATTATTCG 155 N2-ser-4(ok512)-fwd TGGCATGTTGCGAAACTAGA wt:267 mt:367

bas-1(tm351)

167 bas-1(tm351)-fwd TTAGACGTTGGTTGCACGAG 165 bas-1(tm351)-rev CCACCTGAACTGTGGTGATG 166 N2-bas-1(tm351)-fwd TCGTGGGAAATCTTTTTGCT wt:247 mt:354

tph-1 (mg280)

161 tph-1(mg280)-fwd TTTGCGCATAATAAAACAATCAA 162 tph-1(mg280)-rev ACGGAATCCAGTTTTTGCTG 163 N2-tph-1(mg280)-fwd CATCATCCGAACGGAAAACT wt:371 mt:480

egl-3(gk238)

egl-3(gk238)-fwd TCCGTCAAACAGGGAGTTTC

egl-3(gk238)-rev ATTTCGAATTCAGGCAACCA

N2-egl-3(gk238)-fwd GGAGAGGGTAGACGTTGTGC wt:238 mt:389

lin-42(ok2385)

97 lin-42(ok2385)-fwd

98 lin-42(ok2385)-rev

99 N2-lin-42(ok2385)-rev

wt:308 mt:431

zig-5(ok1065)

168 zig-5(ok1065)-fwd GCTCCTCATTCCATTCTGCT

169 zig-5(ok1065)-rev CAGCCGACAATCGTATGGTA

170 N2-zig-5(ok1065)-fwd TGGCTGGGGATTTTCAGTAG wt:237 mt:367

elt-4(ca16)

171 elt-4(ca16)-fwd CAAAAACGCATTCTCAACGA 
Bibliography

172 elt-4(ca16)-rev TTACGCCAGCATGTGGATAG

173 N2-elt-4(ca16)-fwd CACTGGCTCCGTTTCAAAAA

wt:256 mt:428 


\section{Dish tapping device for simultaneous/consecutive stimulation with different strength}

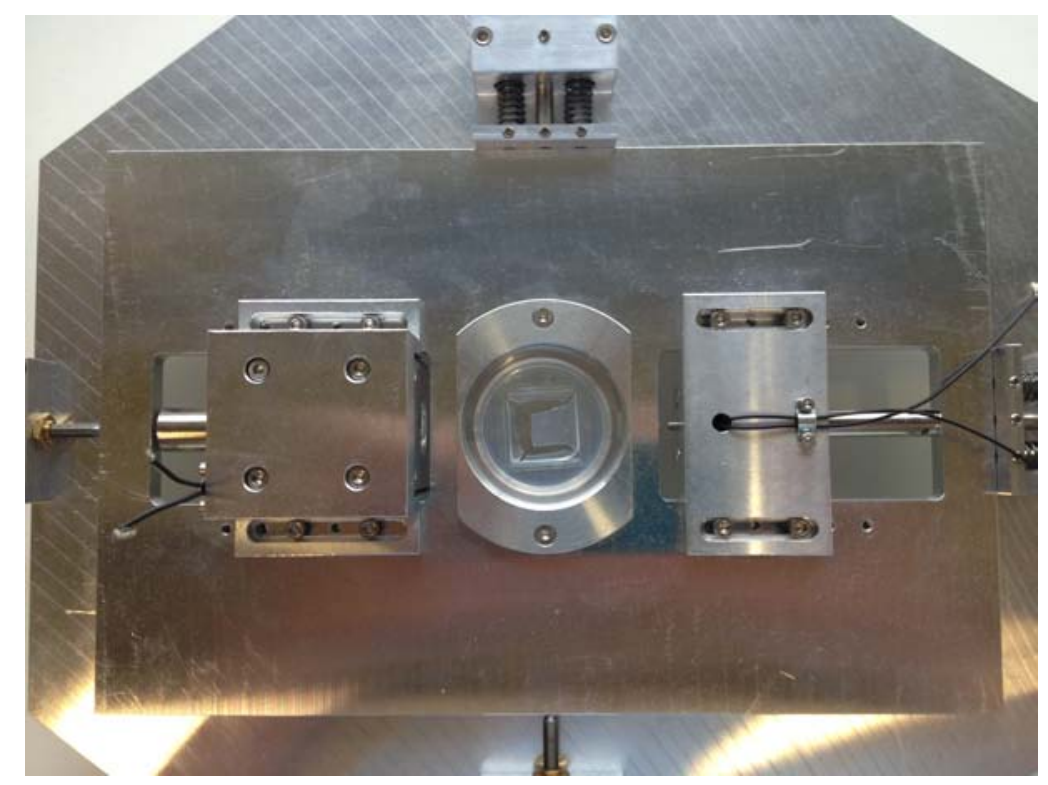

Figure .1: Dish tapping device for simultaneous/consecutive mechanical stimulation with different strength. 


\section{Automated RIS detection}

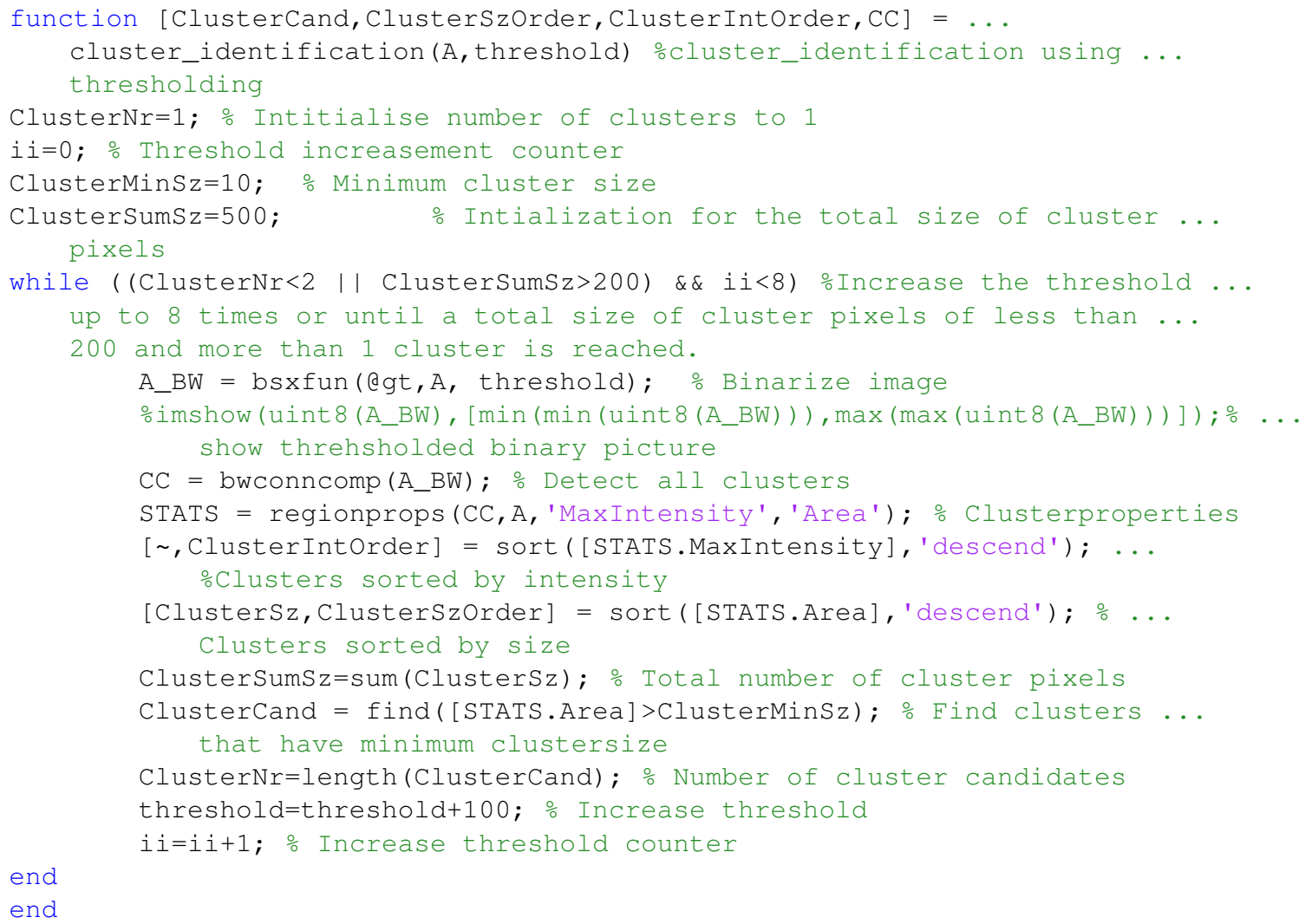


$[\sim, \mathrm{ai}, \mathrm{bi}]=$ intersect(ClusterIntorder, clusterSzOrder) ; \% remove big ... cluster from list

ClustermaxOrder $=$ ClusterIntorder (sort(ai)); \% indices of clusters ... biggers than min clustersize sorted by decreasing cluster intensity cluster_ordered_by_intensity=CC.PixelIdxList (ClustermaxOrder) ; $\%$ sort ... clusters by intensity

$\mathrm{n} \_$cand $=\min (3$, length $($ ClusterCand $)-1) ; \quad \%$ compare maximum the ... highest thre

for $j j=1: n \_c a n d \%$ calculates xy position of high position of peak ... candidate jj

$\left[p k \_i n t \_c a n d(j j), p k \_i n d \_t(j j)\right] \quad=\ldots$

$\max \left(A\left(c l u s t e r \_o r d e r e d \_b y \_i n t e n s i t y\{1, j j\}\right)\right) ; \%$ index of ...

highest value (peak position) for peak candidate jj

$\left[\mathrm{pk} \_\right.$pos_t $(j j, 2), \mathrm{pk} \_$pos_t $\left.(j j, 1)\right]=\ldots$

ind2sub (size (A), cluster_ordered_by_intensity $\{1, j j\}($ pk_ind_t $(j j))$ ); ...

o calculates xy position from index

end

end

pk_pos_cand $=\left[\mathrm{pk} \_p o s \_t(:, 1), \mathrm{pk} \_p o s \_t(:, 2)\right]$;

function [max_distance, RIS_index $]=\ldots$

distance_big_cluster(A,pk_int_cand,pk_pos_cand,pk_pos_biggest_cluster,n_RIS_cand) ...

\% Identifie RIS as peak that has to be within certain distance to the ...

big cluster. Then identify RIS by highest Intensity

pk_dist_max $\quad=\ldots$.

sqrt ( (ones (n_RIS_cand, 1) *pk_pos_biggest_cluster (2)-pk_pos_cand $(:, 2)) \star \ldots$

(ones (n_RIS_cand, 1) *pk_pos_biggest_cluster $(2)$-pk_pos_cand $(:, 2)$ ) +...

(ones (n_RIS_cand, 1) *pk_pos_biggest_cluster $(1)$-pk_pos_cand $(:, 1)$ ) $\ldots$

(ones (n_RIS_cand, 1)*pk_pos_biggest_cluster $(1)$-pk_pos_cand $(:, 1))$ ) ; . .

\% calculates peak distance between RIS candidate and the ...

biggest cluster

RIS_cand $=$ find (pk_dist_max<30 \&\& pk_dist_max $>10)$; $\%$ RIS has to be in the ... range 10-30 pixels to the peak candidate

pk_int_possible = pk_int_cand(RIS_cand); \% Cluster intensities of RIS ... candidates

$[\sim$, pk_ind $]=\max ($ pk_int_possible); \% Index of cluster with highest peak ... intensity

if isempty (pk_ind)==0; $\frac{0}{2}$ if possible RIS cluster has been detected

RIS_index $=p k$ _ind;

max_distance=true;

else

RIS_index $=[]$;

max_distance=false;

end

end

function [boundary,pk_pos_RIS, pk_int_RIS, area_int_RIS] = ...

distance_image_boundary (A, sarea, high, RIS_index,pk_int_cand,pk_pos_cand) ...

\% Checks that RIS is not too close to image boundary and computes the ...

background subtracted intensity of RIS neuron

$[\mathrm{nc}, \mathrm{nr}]=\operatorname{size}(\mathrm{A})$; $\frac{\circ}{\mathrm{a}} \mathrm{image} \operatorname{size}$ 


\section{Bibliography}

pk_pos_RIS=pk_pos_cand(RIS_index, :) ; Peak position of RIS cluster

pk_int_RIS=pk_int_cand(RIS_index); \%Peak intensity of RIS cluster

if (pk_pos_RIS (1) >sarea \& pk_pos_RIS $(1)<($ nr-sarea) \& pk_pos_RIS (2) >sarea \&...

pk_pos_RIS $(2)<($ nc-sarea $))==1$; $\%$ Check that RIS is not too close... to boundary

area =A (pk_pos_RIS (2) -sarea:pk_pos_RIS (2) +sarea,pk_pos_RIS (1) - . .

sarea:pk_pos_RIS(1)+sarea); \% Determine Intensity in ...

surrounding area

low $=($ sarea $* 2+1) \cdot{ }^{\wedge} 2-($ high +1$) ; \quad$ o Number of pixels to be used as ...

background

sort_index =sort (sort (area (:)), 'descend'); \% sort pixel intensities ... of area

high_area =mean(sort_index(1:high)); \% Calculate RIS signal

low_area ...

=mean (sort_index (length (sort_index)-low:length (sort_index)) ); ...

Calculate background signal

area_int_RIS=high_area-low_area; \% Subtract background from RIS signal

boundary=true;

else

boundary=false;

end

function []$=\ldots$

output_RIS_txt_files(pk_pos_lst,pk_int_lst,area_int_lst,ris_not_found_lst,... interval, threshold, sarea, high, filestub)

x0=pk_pos_lst $(1:$ length (pk_pos_lst $(:, 1))-1,:)$; \% Peak positions for the ...

timepoints $t=1-t f i n a l-1$

x1=pk_pos_lst $(2:$ length $($ pk_pos_lst $(:, 1)),:)$; \% Peak positions for the ...

timepoints $t=2-$ tfinal

deltax $=\operatorname{sqrt}\left((x 1(:, 1)-x 0(:, 1)) \cdot{ }^{\wedge} 2+(x 1(:, 2)-x 0(:, 2)) . \wedge 2\right)$; $\%$ Variation of ... peak positions

pk_vel_lst=deltax*1.12/interval; \% velocity of peak positions.1.12 ...

corresponds to px-um translation

filename_base=([filestub, '_threshold_', num2str(threshold), '_sarea_', num2str(sarea), .. . '_high_', num2str(high)]);

filename_pk_pos=( [filename_base, '_RIS_Peak_Pos_list.txt']) ;

save (filename_pk_pos, 'pk_pos_lst', '-ascii');

filename_pk_vel=([filename_base, 'RIS_Peak_Velocity_list.txt']) ;

save (filename_pk_vel, 'pk_vel_lst', '-ascii');

filename_pk_int=( [filename_base, 'RIS_Peak_Int_list.txt']) ;

save (filename_pk_int, 'pk_int_lst', '-ascii');

filename_area_int=([filename_base, 'RIS_Area_Int_list.txt'] );

save (filename_area_int, 'area_int_lst', '-ascii') ;

output_non_recognized_RIS (ris_not_found_lst) ;

filename_ris_not_found=([filename_base, 'RIS_not_found.txt'] ) ;

save (filename_ris_not_found, 'ris_not_found_lst', '-ascii') ;

end 